Doctoral Thesis

\title{
Duality and Integrability in Superstring and Gauge Field Theory
}

\author{
Konstantinos FiLipPAS \\ Supervisors \\ Prof. Carlos Núñez \& \\ Dr. Daniel C. Thompson
}

Particle Physics and Cosmology Theory Group,

Department of Physics, College of Science, Swansea University, Swansea, United Kingdom

Submitted to Swansea University

in fulfilment of the requirements for the degree of

Doctor of Philosophy

July 2021

Copyright: The author, Konstantinos Filippas, 2021. 


\section{Declaration of authorship}

I, Konstantinos FiLIPPAS declare that his thesis titled, "Duality and Integrability in Superstring and Gauge Field Theory" and the work presented in it are based on my research projects in collaboration with Prof. Carlos NunEz and Jeroen van Gorsel. In particular, this thesis is based on:

1] K. Filippas, C. Nunez, J. van Gorsel,

Integrability and holographic aspects of six-dimensional $\mathcal{N}=(1,0)$ superconformal field theories,

Journal of High Energy Physics, 06 (2019) 069,

arXiv:1901.08598 [hep-th].

[2] K. Filippas,

Non-integrability on $A d S_{3}$ supergravity backgrounds,

Journal of High Energy Physics, 02 (2020) 027,

arXiv:1910.12981 [hep-th].

[3] K. Filippas,

Nonintegrability of the $\Omega$ deformation,

Physical Review D 101 (2020) no.4, 046025,

arXiv:1912.03791 [hep-th].

[4] K. Filippas,

Holography for $2 D \mathcal{N}=(0,4)$ quantum field theory,

Physical Review D 103 (2021) no.8, 086003,

arXiv:2008.00314 [hep-th].

The first part of this thesis will use results from [1 3]. The material presented in 4 will be the subject of the second part.

I confirm that:

This work has not previously been accepted in substance for any degree and is not being concurrently submitted for any degree.

Signed:

(candidate)

Date:

$7 / 01 / 2021$ 


\section{Statement 1}

This thesis is the result of my own investigations, except where otherwise stated. Where correction services have been used, the extent and nature of the correction is clearly marked in a footnote(s). Other sources are acknowledged by footnotes giving explicit references. A bibliography is appended.

Signed:

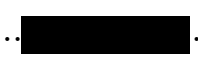

(candidate)

Date:

$7 / 01 / 2021$

\section{Statement 2}

I hereby give consent for my thesis, if accepted, to be available for photocopying and for inter-library loan, and for the title and summary to be made available to outside organizations.

Signed: (candidate)

Date: $7 / 01 / 2021$ 


\section{Acknowledgments}

I am very grateful to my supervisors, Carlos Núñez and Daniel Thompson, for their help and support, as well as for our countless conversations on physics, on the Vivian tower corridors.

I am also grateful to the rest of the Theoretical Particle Physics Group in Swansea for our valuable discussions, especially to Tim Hollowood and Prem Kumar. Moreover, I would like to thank Maurizio Piai and Ken Meissner for their academic support. In a more international basis, I would like to thank Alessandro Tomasiello for our useful correspondence and Dimitris Giataganas for our discussions and his invaluable support during my publications.

Before the pandemics, I was lucky to share a $\mathrm{PhD}$ office with Freya Woods, Stefano Speziali, Mohammad Akhond, Giacomo Piccinini, Sibylle Driezen, Will Roberts and Lucas Schepers. We did not spend so much time together in there, but had our share of fun outside the campus.

I would, especially, like to thank all of my loving friends in Athens, where I spent half of my time as a $\mathrm{PhD}$ candidate. Our friendship helps me remember where I come from, on the inside, so I can understand what I am now.

A great bow also goes to 'Nomads', a workers' assembly in the E/N suburbs of Athens. Our common struggle against all odds, especially during the Covid-19 pandemics, brought new meaning to our community and showed the way things are done.

It goes without saying that the loving oppression and support of my family have been, now more than ever, a dipole of priceless psychoanalysis. We grow and mature together.

Last but not least, words are small when it comes to Fani Christidi. With her, it always feels like either a school of love or a spiritual journey into the unknown. 


\section{Abstract}

In the present thesis, we explore certain aspects of superstring and supersymmetric gauge field theory, independently as well as in the context of the holographic duality.

The first part of the thesis is devoted to classical integrability and, in particular, to certain methods of analytic non-integrability, which are employed on various supergravity vacua. In Chapter 1, we introduce those tools of non-integrability, which consist of choosing an appropriate string embedding and using differential Galois theory on the associated Hamiltonian system. The arena of all this, for the first chapter, is two classes of vacua in massive Type IIA supergravity, all of which are proven to be non-integrable, up to the trivial cases where the vacuum reduces to the Abelian and non-Abelian T-dual of known integrable backgrounds. Differential Galois theory, in this context, reduces to an algebraic form through Kovacic's theorem, the proper use of which, on parametrized differential equations, is clarified in this application.

In Chapter 2, we study integrability on the supergravity vacuum dual to the fieldtheoretical $\Omega$-deformation of super Yang-Mills theory. The deformation manifests itself as turning on a Kalb-Ramond field on the dual supergravity vacuum and, by constructing appropriate string embeddings, we show that this space exhibits non-integrable dynamics. This, in turn, suggests that the $\Omega$-deformation does not preserve classical integrability.

In Chapter 3, we explore integrability on vacua in massive Type IIA supergravity, dual to six-dimensional superconformal quiver field theories. Analytic non-integrability illustrates that all vacua with a warped geometry, between Anti-de-Sitter space and the internal manifold, exhibit complete non-integrability, while in the special case of the unwarped space we prove the opposite to be true. In particular, we show that, besides the integrable dynamics on the symmetric Anti-de-Sitter subspace of the unwarped geometry, the $\sigma$-model on the internal manifold is an integrable deformation of the same model on the symmetric threesphere, ultimately implying classical integrability of bosonic string theory on this special vacuum.

The second part of the thesis is devoted to holography and, in particular, the AdS/CFT duality, which we exploit to study features of certain supersymmetric quantum field theories in two spacetime dimensions. More precisely, in Chapter 4, the final chapter, we study the duality between massive Type IIA supergravity vacua and two-dimensional quiver structures. After categorizing all kinds of gravity solutions, we demystify the ones that seem to reflect anomalous gauge theories. In particular, we prove that there are bound states of D-branes on the boundary of the space which provide the dual quiver theory with exactly the correct amount of matter in order to cancel its gauge anomalies. We also propose that the structure of the field theory should be complemented with additional bifundamental matter and, finally, we construct a BPS string configuration and use the old and new supersymmetric matter to build its dual ultraviolet operator. 


\section{Contents}

\begin{tabular}{ll}
\hline Introduction & 3
\end{tabular}

\begin{tabular}{lll}
\hline I Analytic non-integrability & 11
\end{tabular}

\begin{tabular}{lll}
\hline & Non-integrability on $\mathbf{A d S}_{3}$ vacua & $\mathbf{1 5}$
\end{tabular}

1.1 Introduction . . . . . . . . . . . . . . . . . . . . . . . . 15

$1.2 \quad \mathrm{AdS}_{3} \times \mathrm{S}^{2} \times \mathbb{R} \times \mathrm{CY}_{2}$ vacua $\ldots \ldots \ldots \ldots \ldots \ldots \ldots$

1.3 String dynamics on $\mathrm{AdS}_{3} \times \mathrm{S}^{2} \times \mathbb{R} \ldots \ldots \ldots \ldots \ldots . \ldots \ldots$

1.3 .1 Equations of motion . . . . . . . . . . . . . . . . 22

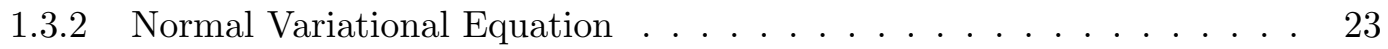

1.4 Class I backgrounds . . . . . . . . . . . . . . . . . . . . . 26

$1.4 .1 \quad$ Abelian T-dual of $\mathrm{AdS}_{3} \times \mathrm{S}^{3} \times \mathrm{T}^{4} \ldots \ldots \ldots \ldots . \ldots 27$

$1.4 .2 \quad$ Non-Abelian T-dual of $\mathrm{AdS}_{3} \times \mathrm{S}^{3} \times \mathrm{T}^{4} \ldots \ldots \ldots \ldots$. . . . . . . . 28

$1.4 .3 \quad$ NVE for the radial dimension . . . . . . . . . . . . . . . . . . . 29

1.4 .4 Case $1 \ldots \ldots \ldots \ldots \ldots$

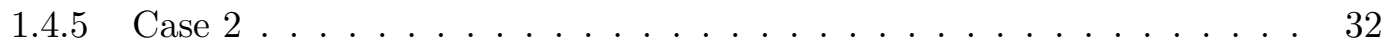

1.5 Class II backgrounds $\ldots \ldots \ldots$. . . . . . . . . . . . . . . . . . . 33

1.5 .1 NVE for the radial dimension . . . . . . . . . . . . . . . . . 34

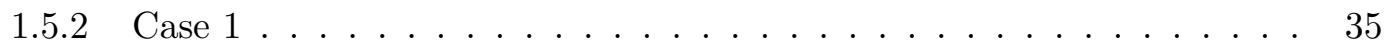

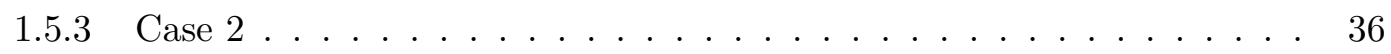

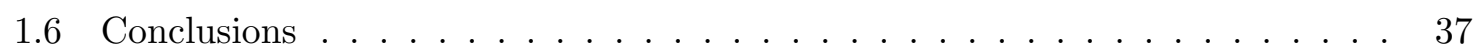

A Differential Galois theory and Kovacic's theorem . . . . . . . . . . . . . . . 38

A.1 The algorithm for Case 1 . . . . . . . . . . . . . . . . . . . . . . . . . . . . .

A.2 The algorithm for Case 2 . . . . . . . . . . . . . . . . 42

B $\quad$ NVEs for the non-Abelian T-dual of $\mathrm{AdS}_{3} \times \mathrm{S}^{3} \times \mathrm{T}^{4} \ldots \ldots \ldots \ldots$. . . . . 44

C Kovacic's theorem on NVEs . . . . . . . . . . . . . . . . . . . . . . . 45

C.1 Case 1 for Class I . . . . . . . . . . . . . . . . . . . . . . . . . 45

C.2 Case 2 for Class I . . . . . . . . . . . . . . . . . . . . . . 46

C.3 Case 1 for Class II . . . . . . . . . . . . . . . . . . . . . . 47 
C.4 Case 2 for Class II . . . . . . . . . . . . . . . . . . . . . 48

$2 \quad$ Non-integrability of the $\Omega$ deformation $\quad 51$

1 Introduction . . . . . . . . . . . . . . . . . . 51

2 The supergravity solution . . . . . . . . . . . . . . . . . 52

3 Symmetries of the vacuum . . . . . . . . . . . . . . . . 53

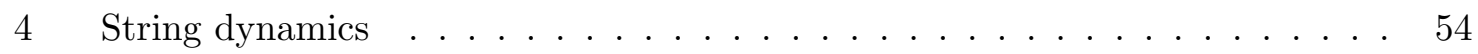

$4.1 \quad$ The first embedding . . . . . . . . . . . . . . . . . . 54

$4.2 \quad$ A simple solution $\ldots \ldots \ldots \ldots \ldots \ldots$

$4.3 \quad$ Fluctuations around the invariant plane . . . . . . . . . . . . . . 59

$4.4 \quad$ A simpler embedding . . . . . . . . . . . . . . . . . . . 60

5 Conclusions . . . . . . . . . . . . . . . . . . . . . . . 63

D Symmetries of the $\Omega$ vacuum $\ldots \ldots \ldots \ldots \ldots$. . . . . . . . . . . . . . . . . . . . . . .

D.1 Constant KVs of $H^{5}$ along the $H_{3} \ldots \ldots \ldots \ldots$. . . . . . . . . 65

D.2 Constant KVs of $\mathrm{dS}_{5}$ along the $H_{3} \ldots \ldots \ldots \ldots \ldots$. . . . . . 67

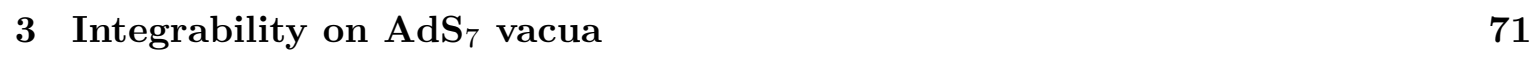

1 Introduction . . . . . . . . . . . . . . . . . . . . . . . 71

$2 \quad$ Six-dimensional SCFT and holography . . . . . . . . . . . . . . . 72

2.1 The dual $\mathrm{AdS}_{7}$ vacua $\ldots \ldots \ldots \ldots \ldots$. . . . . . . . . . . 74

2.2 The quiver gauge theory . . . . . . . . . . . . . . . . . . . 80

$3 \quad$ Non-integrability on the generic $\mathrm{AdS}_{7}$ vacua $\ldots \ldots \ldots \ldots$. . . . . . . . 85

$3.1 \quad$ NVE for a spherical coordinate . . . . . . . . . . . . . . . . . 88

$4 \quad$ Integrability on a special vacuum $\ldots \ldots \ldots \ldots \ldots$. . . . . . . . . . . 89

$4.1 \quad \mathcal{M}^{3}$ as a $\lambda$-deformation of $\mathrm{S}^{3} \ldots \ldots \ldots \ldots$. . . . . . . . . . . 91

4.2 The Lax connection . . . . . . . . . . . . . . . . . . 93

4.3 The dual field theory . . . . . . . . . . . . . . . . . . . . 96

5 conclusions $\ldots \ldots \ldots \ldots \ldots \ldots$

E $\quad$ Analytic non-integrability . . . . . . . . . . . . . . . . . . . . 101

F Integrability on the Symmetric $\sigma$-model $\ldots \ldots \ldots$. . . . . . . . . . 102

\begin{tabular}{ll}
\hline II Holographic duality & 111 \\
\hline
\end{tabular}

\begin{tabular}{lll}
\hline & Holography for two-dimensional QFT & 115
\end{tabular}

$1 \quad$ Introduction . . . . . . . . . . . . . . . . . . . . . . . . . . . . . 115

$2 \quad \mathrm{AdS}_{3}$ massive IIA vacua vs $\mathcal{N}=(0,4)$ theory $\ldots \ldots \ldots \ldots$. . . . . . . 117

$2.1 \quad$ The supergravity solutions $\ldots \ldots \ldots \ldots \ldots$. . . . . . . . . . 117

$2.2 \quad$ Bianchi identities . . . . . . . . . . . . . . . . . 120 


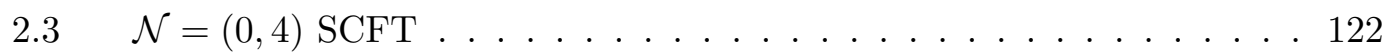

$3 \quad$ Dielectric branes on the boundary $\ldots \ldots \ldots \ldots \ldots$. . . . . . . . . 127

$3.1 \quad$ Linear $u(\rho)$. . . . . . . . . . . . . . . . . . . . . . . . . . . 129

$3.2 \quad$ Constant $u(\rho)$. . . . . . . . . . . . . . . . . . . 137

$4 \quad$ Adding matter in the quiver field theory . . . . . . . . . . . . . . . . . . 141

5 The meson string . . . . . . . . . . . . . . . . . . . . . 144

5.1 A BPS state . . . . . . . . . . . . . . . . . . 144

$5.2 \quad$ An ultraviolet operator . . . . . . . . . . . . . . . . . 145

5.3 Dual mass . . . . . . . . . . . . . . . . . . . . . . . . . . . . . . . . 148

5.4 An alternative operator . . . . . . . . . . . . . . . 151

6 Conclusions . . . . . . . . . . . . . . . . . . . . 151

G Extremal $p$-brane solutions . . . . . . . . . . . . . . . . 153

H Two dimensional $\mathcal{N}=(0,4)$ superfields $\ldots \ldots \ldots \ldots \ldots$

H.1 Field content and action . . . . . . . . . . . . . . . . . . . . . 153

H.2 U(1) R-charge . . . . . . . . . . . . . . . . . 156

I The D8/D4 bound state . . . . . . . . . . . . . . . . . 156

J R-charge of the BPS state . . . . . . . . . . . . . . . . 160

\begin{tabular}{ll}
\hline IIIAppendices and Bibliography & 163
\end{tabular}

$\begin{array}{lll}1 & \text { Supersymmetry in two dimensions } & 165\end{array}$

A Superspace and superfields . . . . . . . . . . . . . . . . 165

A.1 Supersymmetric action terms . . . . . . . . . . . . . . . 167

A.2 Supersymmetric gauge theory . . . . . . . . . . . . . . . . . 168

B $\quad \mathcal{N}=(0,2)$ superfields in two dimensions $\ldots \ldots \ldots \ldots \ldots \ldots$

B.1 Gauge multiplet . . . . . . . . . . . . . . . . 171

B.2 Chiral multiplet . . . . . . . . . . . . . . . . 172

B.3 Fermi multiplet . . . . . . . . . . . . . . . . . 173

B.4 Superpotentials . . . . . . . . . . . . . . . . 174

B.5 $\quad \mathcal{N}=(2,2)$ decomposition $\ldots \ldots \ldots \ldots \ldots \ldots$

C $\quad \mathcal{N}=(0,4)$ superfields in two dimensions $\ldots \ldots \ldots \ldots \ldots$

C.1 Vector multiplets . . . . . . . . . . . . . . . . . 176

C.2 Hypermultiplets and twisted hypermultiplets . . . . . . . . . . 177

C.3 Fermi Multiplets . . . . . . . . . . . . . . . . . . 178

C.4 $\mathcal{N}=(4,4)$ Decomposition . . . . . . . . . . . . . . . . . 179

D R-symmetry . . . . . . . . . . . . . . . . . . 180

\begin{tabular}{ll}
\hline Bibliography & 185
\end{tabular} 


\section{List of Figures}

1.1 An example of a linear function $h_{4,8}(\rho)$. This kind of function is defined piecewise on every interval $\rho \in[2 \pi k, 2(k+1) \pi]$, while it decreases in slope along the $\rho$

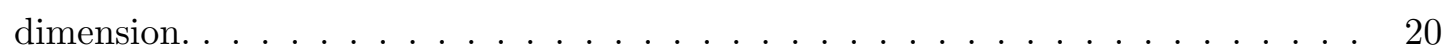

1.2 An example of the linear functions $h_{4,8}(\rho)$ in Class I backgrounds. These piecewise functions decrease in slope along $\rho$ and at least one of them (or both) has to vanish at the end of the dimension, $\rho_{f} . \ldots \ldots \ldots \ldots 27$

1.3 An example of the linear functions $h_{4,8}(\rho)$ in Class II supergravity. These piecewise functions start from $\left.h_{4}\right|_{\rho=0}=\left.h_{8}\right|_{\rho=0}=0$, with $h_{4}>h_{8}$ always, and decrease in slope until they reunite at the end, $\rho_{f}=10 \pi \ldots \ldots \ldots \ldots$. . . . . . . 34

3.1 The Hanany-Witten set-up for the field theory. The vertical lines denote individual Neveu-Schwarz branes extended on the $\left(x_{4}, x_{5}\right)$ space. The horizontal ones D6-branes, that extend on $x_{6}$, in between fivebranes. The crossed-circles represent D8-branes, that extend on the $\left(x_{7}, x_{8}, x_{9}\right)$ directions. All the branes share the Minkowski directions. This realises the isometries $\mathrm{SO}(1,5) \times \mathrm{SO}(3)$. . . . . . . 81

3.2 The quiver corresponding to the Hanany-Witten set-up above. The circle nodes are gauge groups, individual gauge theories, and the square nodes are flavor symmetries. . . . . . . . . . . . . . . . . . . . . 82

3.3 The rank-function $R(z)$ corresponding to the field theory, defined by the ranks of the gauge groups on the quiver structure. . . . . . . . . . . . . 82

3.4 The Hanany-Witten set-up corresponding to the generic field theory studied here. 84

3.5 The quiver corresponding to the generic field theory studied here. . . . . . . . . . 84

$1.1 \quad$ An example of piecewise linear functions $h_{4}, h_{8}$ and of $u$, defining a particular supergravity background. Here, both $h_{4}$ and $h_{8}$ vanish at the endpoints of the $\rho$-dimension. . . . . . . . . . . . . . . . . . . . . . . . . . . . . 119 
1.2 The building block of our quiver field theories. The solid black line represents a $\mathcal{N}=(4,4)$ hypermultiplet, the maroon line a $\mathcal{N}=(0,4)$ hypermultiplet and the dashed line represents a $\mathcal{N}=(0,2)$ Fermi multiplet. Inside the node representing an $\mathrm{SU}(N)$ gauge theory lives a $\mathcal{N}=(4,4)$ vector multiplet. The groups $\mathrm{SU}(P)$, $\mathrm{SU}(Q)$ and $\mathrm{SU}(R)$ can be gauge or global symmetries. . . . . . . . . . . . . 122

1.3 A standard example of a quiver field theory, dual to the proposed family of massive type IIA $\mathrm{AdS}_{3}$ supergravity solutions. This particular quiver theory reflects the solution defined by the functions (1.14) and (1.15). Circle nodes indicate gauge groups while square ones indicate global (flavor) symmetries. This figure is schematic in the sense that flavor nodes are actually far apart in the supergravity limit. . . . . . . . . . . . . . . . . . . . . 125

1.4 All the possible classes of backgrounds defined by a linear function $u(\rho)$ and a non-vanishing function $h_{4}$ or $h_{8}$ at the endpoint $\rho=\rho_{f}$. . . . . . . . . . . 129

1.5 A simplified version of the background given in Figure $1.4 \mathrm{a} \mid$ and its dual quiver theory. Here, besides a linear function $u, h_{8}$ starts and closes with a vanishing value, while $h_{4}$ starts at zero but finishes at a non-zero value. . . . . . . . . . . 130

1.6 This is the actual quiver dual to the background defined by (1.21), (1.22). Here, the extra four-brane flavor node cancels the gauge anomalies for the last $h_{8}(\mathrm{D} 6)$ gauge node. . . . . . . . . . . . . . . . . . 133

\begin{tabular}{|ll|l|l|l|l|}
\hline 1.7 & A simplified version of the background given in Figure & $1.4 \mathrm{~b}$ & and its dual quiver
\end{tabular} theory. Here, besides a linear function $u, h_{4}$ starts and closes with a vanishing value, while $h_{8}$ starts at zero but finishes at a non-zero value. . . . . . . . . . . 134

1.8 This is the actual quiver dual to the background defined by (1.29), (1.30). Here, the extra D2 and D6 flavor nodes cancel the gauge anomalies for the first D6 and the last D2 gauge nodes. . . . . . . . . . . . . . . . . . . . 137

1.9 The representative backgrounds defined by a constant $u(\rho)$ and a non-vanishing $h_{4}$ or $h_{8}$ at either endpoint. The roles of $h_{4}$ and $h_{8}$ may be exchanged in (a) and

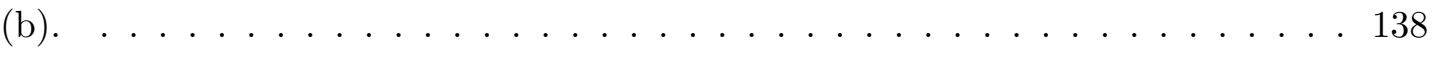

1.10 This is the new dual quiver theory, with additional $\mathcal{N}=(4,4)$ hyper multiplets binding the D4 and D8 flavor nodes with the D2 and D6 gauge nodes, respectively. The already existing $\mathcal{N}=(4,4)$ hyper multiplets are represented with black solid lines, while the new additional ones with orange solid lines. . . . . . . . . . . . 143

1.11 The meson operator $\mathcal{M}$ consists of the supersymmetric multiplets that are highlighted with blue, while the rest of the quiver structure is left blurred. If $k$ and $m$ are the positions of the flavor nodes along the quiver chain, then this operator runs over $m-k+2 \mathcal{N}=(4,4)$ hypermultiplets and $m-k+1 \mathcal{N}=(4,4)$ vector multiplets. Such an operator may also connect D4 with D8 flavors, by jumping through $\mathcal{N}=(0,4)$ hypermultiplets. $\ldots \ldots \ldots \ldots \ldots \ldots$ 


\section{Introduction}





\section{Prologue}

Quantum field theory has been an outstanding framework in understanding particle physics, its agreement with experiment reaching an accuracy of sixteen decimal places. Nonetheless, while there has been much success with quantizing the fields of electromagnetic, strong and weak interactions, gravity still eludes a consistent quantum description. The problem does not occur in quantizing the gravitational field, since an effective field theory of interacting spin-2 particles is perfectly reasonable in larger scales, but in trying to perturbatively renormalize quantum gravity itself [5]. An intuitive way to understand this is through the coupling constant of gravity - that is Newton's constant, $G_{N}$ - which is dimensionful in more than two spacetime dimensions and, by definition, becomes strong in high energies, when perturbation theory is utilized around flat space. Non-renormalizability implies that there should be a replacement theory in small scales - in this case, smaller than Planck's scale, $l_{P}-$ where the quantum effects of gravity are important and the theory is most interesting.

Such a replacement theory is superstring theory [6,7]. The physics of the string shines upon the two-dimensional conformal field theory (CFT) on its worldsheet, which is inherently realized in perturbative terms of the string coupling constant, $g_{s}$. Strings have tension and, hence, string theory has an intrinsic scale in it, the string length $l_{s}$, which is assumed to be of the order of the Plack length, $l_{P}$. String tension, $T=1 / 4 \pi \alpha^{\prime}$ with $\alpha^{\prime}=l_{s}^{2}$, defines worldsheet quantum effects as perturbations along the order of $\alpha^{\prime}$, while the $g_{s}$ expansion describes the way strings couple or split. In perturbative string theory, the fundamental vibrational states that come out off the string perturbation series are the massless particles corresponding to gauge bosons, fermions charged under them and, most notably, gravitons whose equations of motion are the Einstein equations. Along with those massless modes, there is also a dilaton field, $\phi$, whose vacuum expectation value (VEV) practically adjusts the string coupling constant as $g_{s}=e^{\langle\phi\rangle}$. Moreover, boundary conditions of open superstrings yield the existence of spatially-extended, higher-dimensional objects in the theory, on which the open superstrings should end [8]. Those objects are called D-branes and the low-energy limit of the string fluctuations on their worldvolume decouples from gravity and produces gauge field theory.

On the other hand, the full quantum theory demands, also, a complete non-perturbative 
definition, whose expansion would equal the string perturbation series. A key development in this direction is the discovery of certain dualities, symmetries which relate the strong and weak-coupling limits of apparently different superstring theories [9]. Such dualities are the S-duality 10] and the AdS/CFT duality.

The AdS/CFT duality $[11,13]$ is a certain realization of the holographic principle $14-16$. The holographic principle was first inspired by black-hole thermodynamics, where it was shown that the entropy is proportional to the area of the event horizon 17, 18, implying that information of a theory in a space may be encoded on its lower-dimensional boundary. The AdS/CFT duality realizes exactly this kind of situation, while its conceptual grounds gradually emerged from different corners of superstring theory, mainly in an effort to understand the quantum mechanics of black holes [19] and along the physics of $N$ D-branes, which had long been believed to resemble gauge field theory with $\mathrm{SU}(N)$ gauge group at large $N$ 20]. This holographic duality relates the low-energy limit of ten-dimensional superstring theory, that is supergravity, living on $(d+1)$-dimensional Anti-de-Sitter (AdS) space times a compact manifold $\mathcal{M}$, with a (supersymmetric) CFT living in its $d$-dimensional conformal boundary. In this image, the isometries and supersymmetries on the AdS vacuum are seen as the superconformal group of the CFT, while the isometries of $\mathcal{M}$ are translated into an R-symmetry which rotates the supercharges of the dual supersymmetric field theory. String states and their energies are dual to CFT operators and their conformal dimensions, respectively. Additionally, local gauge symmetry of string theory is identified with a global (flavor) symmetry of the CFT at the conformal boundary.

The prime example of the AdS/CFT holography is the duality between the maximally supersymmetric Type IIB supergravity on $\mathrm{AdS}_{5} \times \mathrm{S}^{5}-$ a vacuum which emerges as the near-horizon limit of $N$ D3-branes in flat space - and the four-dimensional supersymmetric Yang-Mills (SYM) theory with $\mathrm{SU}(N)$ gauge group and $\mathcal{N}=4$ supersymmetry.

In order to stay within the supergravity limit of string theory, the radius $R$ of the vacuum has to be large compared to the string length, so that worldsheet quantum effects are suppressed and classical (supergravity) fields dominate the dynamics. This means

$$
R=\sqrt{\alpha^{\prime}}\left(4 \pi g_{s} N\right)^{\frac{1}{4}} \gg \sqrt{\alpha^{\prime}}=l_{s} \quad \Rightarrow \quad 4 \pi g_{s} N \gg 1
$$

where $R$ is the AdS radius. In terms of the field theory coupling constant, $g_{\mathrm{SYM}}^{2} N \gg 1$. Of course, in the same time, quantum string-loop corrections have to be also suppressed in the supergravity approximation, that is $g_{s} \rightarrow 0$, which means $N \rightarrow \infty$. In this limit, the large $N$ limit, $\mathrm{SU}(N)$ gauge theory has as its expansion parameters the effective, t'Hooft coupling $\lambda=g_{\mathrm{SYM}}^{2} N=4 \pi g_{s} N$ and $1 / N$, where planar Feynman diagrams dominate the interactions. In this vacuum, string tension is effectively $T=R^{2} / 2 \pi \alpha^{\prime}$, which means that the expansion 
parameters between string and gauge theory are related as

$$
\lambda=4 \pi^{2} T^{2}, \quad \frac{1}{N}=\frac{g_{s}}{4 \pi^{2} T^{2}} .
$$

In the end, since $g_{s} \rightarrow 0$ and $\lambda \gg 1$, the AdS/CFT duality is a correspondence between weakly-coupled string theory, that is supergravity, and strongly-coupled CFT.

The $\mathrm{AdS}_{5} \times \mathrm{S}^{5}$ vacuum is one of the three maximally supersymmetric vacua of Type IIB superstring theory, along with its flat-space and plane-wave limits [21]. $\operatorname{AdS}_{5} \times \mathrm{S}^{5}$ and its plane-wave (or 'Penrose') limit, together with their dual field theory, have both been the playground for numerous tests on the validity of the AdS/CFT duality, one of the most successful possibly being the work of Berenstein, Maldacena and Nastase (BMN) 22. The first reason for this kind of interest and success in calculations is obviously the large amount of symmetry: lots of geometric isometries and maximal supersymmetry. Maximal supersymmetry reflects cancellation between spinor and bosonic worldsheet effects, yielding an exact vacuum solution; the low-energy equations of motion are exact, receiving no further $\alpha^{\prime}$-corrections. The second reason, which intertwines with the first, is less popular and it is called integrability. Integrability, more or less, is the feature of string and gauge field theory that permits to calculate observables as functions of the coupling constant and, in principle, solve the theory exactly. Planar $\mathcal{N}=4$ SYM theory is such an example [23], where integrability leads to an exact dependence of the scaling dimension $\Delta_{\mathcal{O}}$, of some operator $\mathcal{O}$, in the effective coupling $\lambda$ as $\Delta_{\mathcal{O}}=f(\lambda)$. Computational means at arbitrary $\lambda$ are related, through the AdS/CFT duality, to the regime of free superstrings of arbitrary tension and, hence, a connection is achieved between the perturbative regimes of string and gauge field theory [24]. Of course, this connection due to integrability, as well as integrability itself, is confined to the planar limit, where superstring theory resembles gauge field theory.

Classical, Liouvillian integrability [25,26] is, in general, the ability of a set of differential equations to be solved exactly, by integration. In the context of dynamical systems, in particular, integrability is the kind of situation where the number of first integrals - that is, its conserved quantities - equals the degrees of freedom in the theory; it is those conserved quantities that may be, in principle, used to integrate the differential equations of motion down to an algebraic and solvable form. In field theory, the degrees of freedom are infinite and, for an integrable structure, the same must hold for its conserved quantities, which, moreover, have to be independent and in involution between them, meaning that their Poisson brackets have to vanish.

Despite its exceptionally rich structure, though, integrability is hard to spot throughout the vast variety of superstring vacua or field theories. This is because its presence relies on the existence of a flat Lax connection on the cotangent bundle (phase space) of the theory, 
while, as of yet, there is no standard recipe that provides such a construction. In fact, there is not even an a priori reason to believe that such a connection does exist. Hence, integrable structures are mainly obtained as structure-preserving deformations of known integrable theories, 27 30.

\section{Plan of the Thesis}

Through those strict limitations of classical integrability, analytic non-integrability brings new hope into the struggle of finding novel integrable structures. This is the subject of Part I] of this thesis. In Chapter 1 of this first part, based on [2], we introduce and employ a particular method that is able to spot non-integrable subsectors in a dynamical system. Of course, this suffices in the analysis of integrability, since a non-integrable subsector is enough to declare a theory as non-integrable; integrability is a feature that should be felt throughout the entirety of the dynamical sectors in a system. The method begins by picking a particular bosonic string soliton that is a point-particle along the infinite spatial dimensions of the vacuum and wraps around the compact ones. Such a semi-classical string exhibits secondorder, ordinary, differential equations of motion, instead of partial-differential ones coming from the full dynamics of the bosonic string. This is an important dynamical reduction, since it is this kind of differential equations that may be exploited by differential Galois theory, which can, in turn, decide whether there are Liouvillian solutions or not. After illustrating the method, we apply it on a family of Type IIA supergravity vacua with an $\mathrm{AdS}_{3}$ factor, dual to two-dimensional superconformal field theories (SCFTs) that flow to quiver theories with $\mathcal{N}=(0,4)$ supersymmetry in the ultraviolet (UV), where we deduce that all vacua are non-integrable except a couple of trivial, limiting cases.

In Chapter 2, based on [3], we employ the same method of non-integrability on the supergravity vacuum dual to the field theoretical $\Omega$-deformation of $\mathcal{N}=4$ SYM theory. In this case, life is simpler and we do not have to use differential Galois theory, since we find exact solutions for the equations of motion, in a straightforward manner, which are not Liouvillian. Subsequently, we deduce that the vacuum and, thus, its dual $\Omega$-deformation are classically non-integrable structures.

In Chapter 3, based on [1], analytic non-integrability is applied on a family of Type IIA supergravity vacua with an $\mathrm{AdS}_{7}$ factor, dual to six-dimensional SCFTs that flow to quiver field theories with $\mathcal{N}=(0,1)$ supersymmetry in the infrared (IR). The whole family is shown to be non-integrable, except one special case where the AdS part of the geometry unwarps from the internal manifold. In this special case, the internal manifold is proven to be an integrable deformation of $\mathrm{S}^{3}$, where the bosonic string is classically integrable. Using the fact that AdS is also a symmetric space - hence exhibiting integrable string dynamics - a Lax connection is produced for the dynamical system and the special vacuum is ultimately declared to be integrable. 
Finally, Part II] of this thesis, based on [4], is devoted to certain aspects of the AdS/CFT duality. In particular, we bring again the $\mathrm{AdS}_{3}$ supergravity family of Chapter 1 back on the table and focus on some classes of vacua that seem to reflect anomalous gauge theories. We then study those cases in their dual gravity backgrounds and show that their gauge anomalies, in fact, cancel and that the construction is, thus, perfectly consistent, while we test holography by proposing some dual operators for a particular state of the bosonic string. As a side-tool for Part II, an Appendix is provided in the end of the thesis, where supersymmetry for two-dimensional gauge theory is reviewed. 





\section{Part I}

\section{Analytic non-integrability}





\section{Part I preface}

In this first part, we employ our tools of analytic non-integrability on a variety of vacua in string theory. The part is divided into three chapters, each dedicated to one of the publications $[1-3]$.

In Chapter 1, following [2], we investigate classical integrability on two classes of $\mathrm{AdS}_{3}$ backgrounds in massive Type IIA supergravity, 31,34 . Those vacua are of the form $\mathrm{AdS}_{3} \times \mathrm{S}^{2}$ $\times \mathbb{R} \times \mathrm{CY}_{2}$, they preserve small $\mathcal{N}=(0,4)$ supersymmetry and are associated with D8-D6-D4 -D2 Hanany-Witten brane set-ups. We choose an appropriate string embedding and use differential Galois theory on its associated Hamiltonian system, intending to produce the conditions under which Liouvillian solutions may occur. By constraining the parameters of the system according to the consistency of the associate brane set-ups we prove that no such conditions exist, yielding the complete non-integrability of those vacua. That is, up to the trivial cases where the background reduces to the Abelian and non-Abelian T-dual of $\mathrm{AdS}_{3} \times \mathrm{S}^{3} \times \mathrm{T}^{4}$.

In Chapter 2, following [3], we study classical integrability on the supergravity vacuum dual to the field theoretical $\Omega$-deformation of $\mathcal{N}=4$ SYM theory, [35]. The deformation manifests itself as turning on a Kalb-Ramond field on the (Euclidean) $\mathrm{AdS}_{5} \times \mathrm{S}^{5}$, while the associated $H_{3}$ flux ignores half of the geometric isometries. By constructing appropriate string embeddings that incorporate the essential $H_{3}$ flux contribution on this background, we study their fluctuations through the associated Hamiltonian systems. Each and every case demonstrates that the string exhibits non-integrable dynamics, which in turn suggests that the $\Omega$-deformation does not preserve classical integrability.

In Chapter 3 , following [1], we explore classical integrability on a family of $\mathrm{AdS}_{7}$ vacua in massive Type IIA supergravity, 36 43. Those vacua consist of a warped $\mathrm{AdS}_{7} \times \mathcal{M}^{3}$ geometry, where $\mathcal{M}^{3}$ is isomorphic to $\mathrm{S}^{3}$, they are associated with D8-D6 Hanany-Witten brane set-ups and are dual to a class of six-dimensional superconformal quiver field theories with $\mathcal{N}=(1,0)$ supersymmetry. While the study of a particular bosonic string soliton illustrates that all vacua with a warped geometry exhibit complete non-integrability, in the special case of the unwarped $\mathrm{AdS}_{7} \times \mathcal{M}^{3}$ space we prove the opposite to be true. That is, we observe that the Wess-Zumino-Witten model on $\mathcal{M}^{3}$ is an (integrable) $\lambda$-deformation of the same model on $\mathrm{S}^{3}$, ultimately showing that the Neveu-Schwarz (NS) sector of the string $\sigma$-model on this special vacuum is classically integrable. 



\section{Chapter 1}

\section{Non-integrability on $\mathrm{AdS}_{3}$ vacua}

\section{$1.1 \quad$ Introduction}

Integrability possesses an essential role in modern field theory. Not only it reveals a rich structure of conserved quantities that shape the physics of the system, but it also states that the theory is solvable for any choice of the coupling constant. Since holography relates the worldsheet theory of the superstring to a quantum field theory, integrable structures in string theory have won a prominent role in leading the way to new integrable gauge theories, 24 26 44. Even the most successful calculations on the standard AdS/CFT correspondence, between $\mathrm{AdS}_{5} \times \mathrm{S}^{5}$ supergravity and $\mathcal{N}=4$ super Yang-Mills theory, rely on the complete integrability in the planar limit of the system.

However, spotting integrable structures can prove to be quite a challenging task. Integrability depends on the existence of a Lax connection on the cotangent bundle of the theory, while no standard recipe is provided to acquire such a construction. In fact, there is not even an a priori reason to decide whether such a connection does exist. That is, unless we acknowledge the theory to be non-integrable. Therefore, integrable systems are mainly obtained as structure-preserving deformations of known integrable theories, $27-30]$.

Through the limitations of the classic methods of integrability, analytic non-integrability manifests itself in a dialectic way. Considering Hamiltonian systems of equations, analytic non-integrability makes use of Galois theory on differential equations to produce a statement on the structure of these systems. The arguments of differential Galois theory on second order, ordinary, linear differential equations were brought to an algebraic form by Kovacic [45, who also provided an explicit algorithm that produces the Liouvillian solutions of such equations, if any.

In terms of supergravity, we choose a string embedding that produces the kind of differential equations of motion that can be examined under Kovacic's theorem, [1, 46 62]. Since an integrable theory has all of its dynamical sectors integrable, then every possible string configuration must echo integrable dynamics. That is, in the planar limit of the effective parameters in the theory. Even a single sector exhibiting non-integrable behavior is enough 
to declare a supergravity vacuum as non-integrable. Therefore, we choose an embedding complicated enough to provoke the possibly non-integrable structure of the background but, at the same time, simple enough to produce the kind of differential equations we can examine under differential Galois theory.

On another approach, S-matrix factorization on the worldsheet theory of the string was used to provide certain conditions of non-integrability, [63 66], while very recently a reconciliation began to arise between both non-integrability tools, 67.

The present section, which employs differential Galois theory, comes as advertised and proves a recently discovered $\mathrm{AdS}_{3}$ supergravity vacua family, 31 , 34, to be classically nonintegrable. That is, up to the trivial cases where the background reduces to the Abelian and non-Abelian T-dual of $\mathrm{AdS}_{3} \times \mathrm{S}^{3} \times \mathrm{T}^{4}$. These massive IIA vacua are classified in 31 in two distinct classes of backgrounds, from which we consider certain solutions of the form $\mathrm{AdS}_{3} \times \mathrm{S}^{2} \times \mathbb{R} \times \mathrm{CY}_{2}$ as in 33 . The solutions preserve small $\mathcal{N}=(0,4)$ supersymmetry and are associated with D8-D6-D4-D2 Hanany-Witten brane set-ups, [68]. Holography suggests these backgrounds to be dual to two-dimensional quiver quantum field theories. Special holographic features of the $\mathrm{AdS}_{3} / \mathrm{CFT}_{2}$ duality over the solutions we consider were studied in 69. Other warped massive IIA $\mathrm{AdS}_{3}$ supergravities, associated with similar brane set-ups, were introduced in 70,71, while an extensive study of two-dimensional $\mathcal{N}=(0,4)$ quiver gauge theories was performed in 72 .

At the same time, this section also aims to clarify the proper use of Kovacic's theorem on parametrized differential equations. In particular, we emphasize that failure of Kovacic's algorithm - which is implemented in every algebra software - on a parametrized equation does not imply absence of Liouvillian solutions. It just states that not all choices of the parameters lead to an integrable equation. It does certainly not say that there are no particular selections among them that lead to integrability. Hence, if full generality on the parameters is demanded, then failure of Kovacic's algorithm indeed declares the nonintegrability of the system. On the other hand, if the problem allows its parameters to be adjustable, no such statement can be made.

In the latter case, we must enforce the full power of Kovacic's theorem and go over its analytic algorithm by hand. If special parameter selections (that lead to an integrable structure) exist, then Kovacic's analytic algorithm will find them all, along with their associated solutions. If there are no such selections, then we can safely declare our system as non-integrable.

This is exactly what happens in our case. The $\mathrm{AdS}_{3}$ supergravity family we consider is defined on general parameters whose adjustment equals picking different supergravity backgrounds. Therefore, the failure of Kovacic's algorithm here just states that not all possible backgrounds are integrable. It does not say that there are no integrable ones, among the whole family. But this is to be expected. It is the possible special combinations 
of these parameters, i.e. the particular supergravity backgrounds, that we are interested in. By demanding consistency on the supergravity brane set-ups, we show that the parameters are constrained in such a way that no integrable backgrounds of this supergravity family can exist. That is, as restated, up to the trivial cases where the background reduces to the Abelian and non-Abelian T-dual of $\mathrm{AdS}_{3} \times \mathrm{S}^{3} \times \mathrm{T}^{4}$.

The plan of this section is as follows. In Section 1.2, we present the backgrounds of the form $\mathrm{AdS}_{3} \times \mathrm{S}^{2} \times \mathbb{R} \times \mathrm{CY}_{2}$ in a general manner and give a qualitative picture of their features. In Section 1.3, we construct our string embedding and produce its equations of motion. By choosing a simple solution of these equations, we find the dynamical fluctuations around it. In Sections 1.4 and 1.5, we independently study the two classes of the $\mathrm{AdS}_{3}$ backgrounds, by applying differential Galois theory on their associated fluctuations. Each class corresponds to a different kind of brane set-ups and, thus, exhibits different restrictions on its background parameters. By employing Kovacic's analytic algorithm, we show that in both supergravity classes these restrictions forbid integrability for all the possible backgrounds. Finally, in Section 1.6, we summarize our results and give a review of our method as a concrete nonintegrability tool.

\section{$1.2 \quad \mathrm{AdS}_{3} \times \mathrm{S}^{2} \times \mathbb{R} \times \mathrm{CY}_{2} \quad$ vacua}

Let us outline the supergravity vacua that we are about to consider. It is essential to understand the basic aspects of these backgrounds, since it is the physical restrictions on their parameters that will ultimately decide the fate of their (non-) integrability.

The massive IIA supergravity vacua first constructed in [31] split in two distinct classes, Class I and II. From each class, we pick the solutions of the form $\mathrm{AdS}_{3} \times \mathrm{S}^{2} \times \mathbb{R} \times \mathrm{CY}_{2}$ as in [33]. From now on, Class I and II will indicate this particular choice. Both classes have NS-NS sector, in string frame,

$$
\begin{gathered}
\mathrm{d} s^{2}=f_{1} \mathrm{~d} s_{\mathrm{AdS}_{3}}^{2}+f_{2} \mathrm{~d} s_{\mathrm{S}^{2}}^{2}+\frac{\mathrm{d} \rho^{2}}{f_{1}}+f_{3} \mathrm{~d} s_{\mathrm{CY}_{2}}^{2}, \\
B_{2}=f_{4} \operatorname{vol}_{\mathrm{S}^{2}}, \quad e^{-\Phi}=f_{5},
\end{gathered}
$$

where $u, h_{4}, h_{8}$ are functions of the coordinates $\left\{\rho, \mathrm{CY}_{2}\right\}$, left to be defined. The RR sector, consisting of $F_{0}, F_{2}$ and $F_{4}$, will not be needed here. These backgrounds enjoy a bosonic $\mathrm{SL}(2) \times \mathrm{SU}(2)$ isometry, they have eight supercharges and were proposed to be dual to $\mathcal{N}=(0,4)$ CFTs in two dimensions. Here we will consider the solutions on which the symmetries of $\mathrm{CY}_{2}$ are globally respected. This restricts the internal Calabi-Yau manifold to be either 


$$
\mathrm{CY}_{2}=\mathrm{T}^{4} \quad \text { or } \quad \mathrm{CY}_{2}=\mathrm{K} 3
$$

and the warp factors to be $f_{i}=f_{i}(\rho)$, i.e. $u=u(\rho), h_{4}=h_{4}(\rho)$ and $h_{8}=h_{8}(\rho)$. The warp factor dependence on these functions will be specified for each supergravity class accordingly in the sections to follow. Preservation of the $\mathcal{N}=(0,4)$ supersymmetry and the Bianchi identities imply

$$
u^{\prime \prime}(\rho)=0, \quad h_{4}^{\prime \prime}(\rho)=h_{8}^{\prime \prime}(\rho)=0,
$$

respectively. Therefore, all the defining functions are linear in $\rho$ and we parametrize them as

$$
u(\rho)=c_{2}+c_{3} \rho, \quad h_{4}(\rho)=c_{4}+c_{5} \rho, \quad h_{8}(\rho)=c_{1}+F_{0} \rho,
$$

where all $c_{i}$ are real. For the new solutions to be associated with Hanany-Witten brane set-ups, these funtions are defined piecewise on the intervals $\rho \in[2 \pi k, 2 \pi(k+1)], k \in \mathbb{Z}$. Imposing that the functions vanish at $\rho=0$ where the space begins, we get

$$
\begin{gathered}
h_{4}(\rho)=\Upsilon\left\{\begin{array}{cc}
\frac{c_{5}^{0}}{2 \pi} \rho & 0 \leq \rho \leq 2 \pi \\
c_{4}^{k}+\frac{c_{5}^{k}}{2 \pi}(\rho-2 \pi k) & 2 \pi k \leq \rho \leq 2 \pi(k+1) \\
c_{4}^{P}+\frac{c_{5}^{P}}{2 \pi}(\rho-2 \pi P) & 2 \pi P \leq \rho \leq 2 \pi(P+1)
\end{array}\right. \\
h_{8}(\rho)=\left\{\begin{array}{cc}
\frac{F_{0}^{0}}{2 \pi} \rho & 0 \leq \rho \leq 2 \pi \\
c_{1}^{k}+\frac{F_{0}^{k}}{2 \pi}(\rho-2 \pi k) & 2 \pi k \leq \rho \leq 2 \pi(k+1) \\
c_{1}^{P}+\frac{F_{0}^{P}}{2 \pi}(\rho-2 \pi P) & 2 \pi P \leq \rho \leq 2 \pi(P+1)
\end{array}\right.
\end{gathered}
$$

and $u(\rho)=\frac{c_{3}}{2 \pi} \rho . \quad \Upsilon$ is just a constant that may be normalized conveniently. The first derivatives of $h_{4}, h_{8}$ present discontinuities at $\rho=2 k \pi$ where D4 and D8 branes are located 1 while $u^{\prime \prime}=0$ across all intervals as dictated by global supersymmetry. The discontinuities in the RR sector, that are interpreted as localized branes along $\rho$, modify the Bianchi identities appropriately with delta functions. Note that in order for supergravity to be trustable, $\left\{c_{1}, . ., c_{5}, F_{0}, P\right\}$ have to be large.

Continuity of the NS-NS sector implies continuity of the $h_{4}, h_{8}$ functions across the $\rho$

\footnotetext{
${ }^{1}$ We omit to present the explicit dependence of the RR sector to $h_{4}, h_{8}$ (which, like the NS sector, differs for each class of vacua) to avoid unnecessary formulas. However, the restless reader is prompted to 31 . for details or to 33 for a clearer review.
} 
intervals. This leads to

$$
c_{4}^{k+1}=c_{4}^{k}+c_{5}^{k}, \quad c_{1}^{k+1}=c_{1}^{k}+F_{0}^{k},
$$

which in turn gives

$$
c_{4}^{k+1}=\sum_{j=0}^{k} c_{5}^{j}, \quad c_{1}^{k+1}=\sum_{j=0}^{k} F_{0}^{j} .
$$

\section{Page charges}

In order to gain a better feel on the parameters $\left\{c_{1}, . ., c_{5}, F_{0}\right\}$ we consider, as an example, the RR Page charges of Class I supergravity vacua, in the intervals $[2 \pi k, 2 \pi(k+1)]$.

As opposed to other kinds of D-brane charges, the Page charge is not gauge invariant but is localized, conserved and quantized [73]. Hence, through those virtues, the Page charge informs us of the number of D-branes we are dealing with in a situation. A Dp-brane is charged under

$$
Q_{D p}=(2 \pi)^{p-7} g_{s} \alpha^{\prime \frac{p-7}{2}} \int_{\Sigma_{8-p}} \hat{F}_{8-p}
$$

where $\hat{F}=e^{-B_{2}} \wedge F$ is the Page flux, $g_{s}$ is the string coupling constant and $\alpha^{\prime}$ is associated to the squared length of the string. $\Sigma_{8-p}$ is a $(8-p)$-dimensional compact manifold which envelops the Page charge. In our set-up, for $\alpha^{\prime}=g_{s}=1$, the Page charges read 2

$$
\begin{aligned}
Q_{D 8} & =F_{0}^{k}, & Q_{D 6} & =\frac{1}{2 \pi} \int_{\mathrm{S}^{2}} \hat{F}_{2} \sim c_{1}^{k}, \\
Q_{D 4} & =\frac{1}{8 \pi^{3}} \int_{\mathrm{CY}_{2}} \hat{F}_{4} \sim c_{5}^{k}, & Q_{D 2} & =\frac{1}{32 \pi^{5}} \int_{\mathrm{CY}_{2} \times \mathrm{S}^{2}} \hat{F}_{6} \sim c_{4}^{k}
\end{aligned}
$$

and $Q_{N S}=\frac{1}{4 \pi^{2}} \int_{\rho \times S^{2}} H_{3}=P+1$. Therefore, the quantities in the right hand side of the above equations must be integers.

A study of the Bianchi identities reveals that no explicit D2 and D6 branes are present in the geometry, just their fluxes $3^{3}$ This associates their amount, $c_{4}^{k}$ and $c_{1}^{k}$ respectively, with the ranks of the (color) gauge groups in the dual field theory. On the other hand, as restated, D8 and D4 branes do exist in the geometry and modify the Bianchi identities

\footnotetext{
${ }^{2} F_{0}^{k}$ is $F_{0}$ in the $k$-th interval. Whenever we loose the $k$ subscript we will mean $F_{0}^{k}$.

${ }^{3}$ This is true when the worldvolume gauge field on the D8, D4 branes is absent. When it is on, there is D6 and D2 flavor charge induced on the D8's and D4's. See the appendix B of [33. for details.
} 


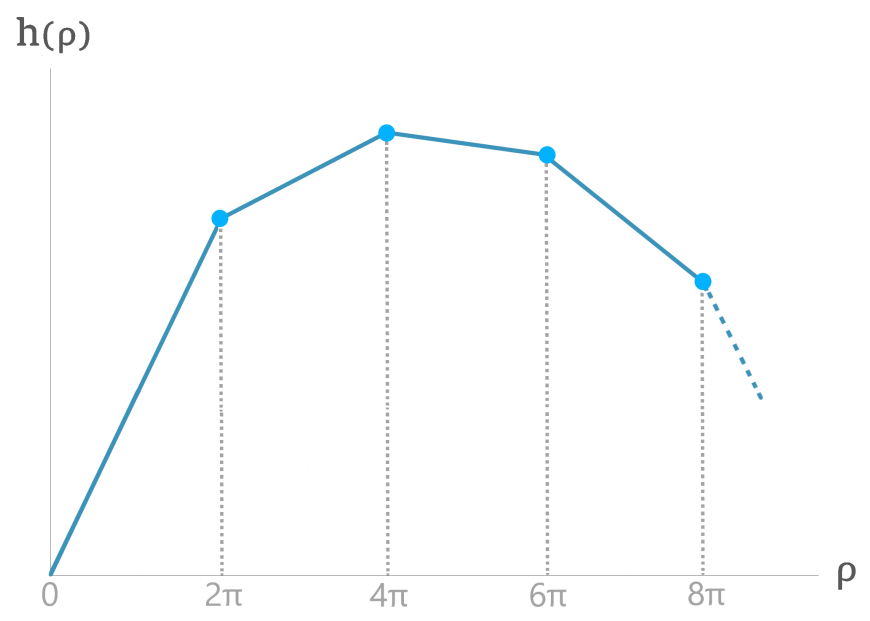

Figure 1.1: An example of a linear function $h_{4,8}(\rho)$. This kind of function is defined piecewise on every interval $\rho \in[2 \pi k, 2(k+1) \pi]$, while it decreases in slope along the $\rho$ dimension.

by a delta function. Thus, $F_{0}^{k}$ and $c_{5}^{k}$ are associated with the ranks of the (flavor) global symmetries of the dual field theory.

\section{Gauge anomaly cancellation}

Realizing the $h_{4}$ and $h_{8}$ pieces across the $\rho$ dimension as blocks of gauge and flavor groups in the dual two-dimensional quantum field theory, we assembly them to quiver gauge theories. Then, cancellation of their gauge anomalies implies

$$
N_{D 8}^{[k-1, k]}=F_{0}^{k-1}-F_{0}^{k}, \quad N_{D 4}^{[k-1, k]}=c_{5}^{k-1}-c_{5}^{k}
$$

For the $h_{4}, h_{8}$ functions this translates to decreasing slope ${ }^{5} c_{5}^{k}$ and $F_{0}^{k}$ respectively, as $\rho$ increases. Thus, any of these functions draws a piecewise linear curve of decreasing slope, as in Figure 1.1 .

While the present section provides a consistent summary of these particular $\mathrm{AdS}_{3}$ supergravity vacua and their dual quiver field theory, the reader is prompted to [31] for details on the construction of the solutions, to [32] for an overview and to [33] for a deeper dive into the quiver realization.

\footnotetext{
${ }^{4}$ The rank is a positive number. If the slope is negative, that is related to the orientation of the branes.

${ }^{5}$ Or slopes that remain the same across intervals, giving no flavor branes between them.
} 


\subsection{String dynamics on $\mathrm{AdS}_{3} \times \mathrm{S}^{2} \times \mathbb{R}$}

The bosonic string dynamics is reflected on the Polyakov action, that is a non-linear $\sigma$-model, which in conformal gauge reads

$$
S_{P}=-\frac{1}{4 \pi \alpha^{\prime}} \int_{\Sigma} \mathrm{d}^{2} \sigma \partial_{a} X^{\mu} \partial_{b} X^{\nu}\left(g_{\mu \nu} \eta^{a b}+B_{\mu \nu} \epsilon^{a b}\right)
$$

where the string coordinates $X^{\mu}(\tau, \sigma)$ equation of motion is supplemented by the Virasoro constraint $T_{a b}=0$, where the worldsheet energy-momentum tensor is given by

$$
T_{a b}=\frac{1}{\alpha^{\prime}}\left(\partial_{a} X^{\mu} \partial_{b} X^{\nu} g_{\mu \nu}-\frac{1}{2} \eta_{a b} \eta^{c d} \partial_{c} X^{\mu} \partial_{d} X^{\nu} g_{\mu \nu}\right)
$$

We desire a string embedding that produces ordinary differential equations as its equations of motion, so that we can apply differential Galois theory. In order for the differential equations of motion to end up ordinary (and not partial) the string coordinates must be $X^{\mu}=X^{\mu}(\tau)$ or $X^{\mu}=X^{\mu}(\sigma)$, where $\tau, \sigma$ are the worldsheet coordinates. Since the search of (non-) integrability requires bringing dynamics to the test, we like our soliton to have as much stringy character as possible, according always to the above restriction $X^{\mu}=X^{\mu}(\sigma)$. Thus, we wrap it around all cyclic coordinates available.

Both Class I and II of the $\mathrm{AdS}_{3}$ supergravity vacua we consider consist of the NS-NS sector, in the string frame,

$$
\begin{aligned}
\mathrm{d} s^{2} & =f_{1} \mathrm{~d} s_{\mathrm{AdS}_{3}}^{2}+f_{2} \mathrm{~d} s_{\mathrm{S}^{2}}^{2}+\frac{\mathrm{d} \rho^{2}}{f_{1}}+f_{3} \mathrm{~d} s_{\mathrm{CY}_{2}}^{2}, \\
B_{2} & =f_{4} \operatorname{vol}_{\mathrm{S}^{2}}, \quad e^{-\Phi}=f_{5},
\end{aligned}
$$

where $f_{i}=f_{i}(\rho)$ are the various warp factors, left undefined for each supergravity class to be separately examined, and $\operatorname{vol}_{\mathrm{S}^{2}}=\sin \chi \mathrm{d} \chi \wedge \mathrm{d} \xi$. If global $\mathrm{AdS}_{3}$ and $\mathrm{S}^{2}$ with unit radii are expressed as

$$
\begin{aligned}
\mathrm{d} s_{\mathrm{AdS}_{3}}^{2} & =-\cosh ^{2} r \mathrm{~d} t^{2}+\mathrm{d} r^{2}+\sinh ^{2} r \mathrm{~d} \phi^{2}, \\
\mathrm{~d} s_{\mathrm{S}^{2}}^{2} & =\mathrm{d} \chi^{2}+\sin ^{2} \chi \mathrm{d} \xi^{2},
\end{aligned}
$$

then we set up our string embedding to be 


$$
\begin{gathered}
t=t(\tau), \quad r=r(\tau), \quad \phi=\nu \sigma, \\
\rho=\rho(\tau), \quad \chi=\chi(\tau), \quad \xi=\kappa \sigma,
\end{gathered}
$$

where we wrapped the string $\nu$ and $\kappa$ times around the $\phi$ coordinate and the $\xi$ dimension, respectively. $\mathrm{CY}_{2}$ dynamics was left out of the game, since it won't be eventually needed in the hunt of non-integrability. Note that it is the wrapping that provides the stringy, non-trivial behavior to the configuration. Without it we would just have point particle dynamics. Indeed, one of these winding modes will play a crucial role later on when we enforce differential Galois theory.

\subsubsection{Equations of motion}

Instead of the action (1.11), it is more convenient working with its associated Langrangian density

$$
\mathcal{L}=f_{1}\left(\cosh ^{2} r \dot{t}^{2}-\dot{r}^{2}+\nu^{2} \sinh ^{2} r\right)-\frac{\dot{\rho}^{2}}{f_{1}}-f_{2}\left(\dot{\chi}^{2}-\kappa^{2} \sin ^{2} \chi\right)+2 \kappa f_{4} \sin \chi \dot{\chi},
$$

where the dot implies derivation wrt the worldsheet time $\tau$. For our particular string embedding, the equations of motion for this Lagrangian are equivalent to those of the $\sigma$-model and read

$$
\begin{aligned}
& \dot{t}=\frac{E}{f_{1} \cosh ^{2} r}, \\
& \ddot{r}=-\frac{\nu^{2} f_{1}^{2} \sinh 2 r+2 E^{2} \tanh r \operatorname{sech}^{2} r+2 f_{1} f_{1}^{\prime} \dot{r} \dot{\rho}}{2 f_{1}^{2}}, \\
& \ddot{\chi}=-\kappa^{2} \cos \chi \sin \chi+\frac{\dot{\rho}\left(-f_{2}^{\prime} \dot{\chi}+\kappa f_{4}^{\prime} \sin \chi\right)}{f_{2}}, \\
& \ddot{\rho}=\frac{f_{1}^{\prime}\left(-E^{2} \operatorname{sech}^{2} r+f_{1}^{2}\left(-\nu^{2} \sinh ^{2} r+\dot{\rho}^{2}\right)\right)+f_{1}^{2}\left(\left(-\kappa^{2} \sin ^{2} \chi+\dot{\chi}^{2}\right) f_{2}^{\prime}-2 \kappa f_{4}^{\prime} \sin \chi \dot{\chi}\right)}{2 f_{1}},
\end{aligned}
$$

where the dash on $f_{i}$ 's implies derivation wrt their argument $\rho$. Notice that we have replaced 
the equation of motion for $t$ into the rest of the equations. These equations of motion are constrained by the worldsheet equation of motion, i.e. the Virasoro constraint

$$
\begin{aligned}
2 T_{\tau \tau} & =2 T_{\sigma \sigma}=f_{1}\left(-\cosh ^{2} r \dot{t}^{2}+\dot{r}^{2}+\nu^{2} \sinh ^{2} r\right)+\frac{\dot{\rho}^{2}}{f_{1}}+f_{2}\left(\dot{\chi}^{2}+\kappa^{2} \sin ^{2} \chi\right)=0 \\
T_{\sigma \tau} & =0
\end{aligned}
$$

This constraint holds regardless of the equations of motion and, thus, it is a primary constraint. The energy-momentum tensor is preserved on shell, $\nabla_{a} T^{a b}=0$, since $\partial_{\tau} T_{\tau \tau}=$ $\partial_{\sigma} T_{\sigma \sigma}=0$ on the equations of motion (1.17). Note, also, that the compliance of the worldsheet constraints with the equations of motion yield the consistency of our embedding.

In order to deeply appreciate our method and get a better grip on its physics, we break on through to the Hamiltonian formulation, by defining the conjugate momenta

$$
p_{t}=2 f_{1} \cosh ^{2} r \dot{t}, \quad p_{r}=-2 f_{1} \dot{r}, \quad p_{\chi}=-2 f_{2} \dot{\chi}+2 \kappa f_{4} \sin \chi, \quad p_{\rho}=-\frac{2 \dot{\rho}}{f_{1}}
$$

and the Hamiltonian density

$$
\mathcal{H}=\frac{p_{t}^{2}}{4 f_{1} \cosh ^{2} r}-\frac{p_{r}^{2}}{4 f_{1}}-\frac{p_{\rho}^{2}}{4\left(f_{1}\right)^{-1}}-\frac{\left(p_{\chi}-2 \kappa f_{4} \sin \chi\right)^{2}}{4 f_{2}}-\kappa^{2} f_{2} \sin ^{2} \chi-\nu^{2} f_{1} \sinh ^{2} r .
$$

In this language, the Virasoro constraint is $\mathcal{H}=0$. Hamilton's equations on $\mathcal{H}$ and $p_{i}$ coincide, of course, with the Euler-Lagrange equations of motion (1.17). Therefore, our string dynamics problem reduces to that of a particle in a non-trivial potential. In particular, the effective mass is defined by geometry through the kinetic terms, while the winding modes in the string perspective are realized as a potential on the particle.

\subsubsection{Normal Variational Equation}

While a system of involved differential equations of motion is unattractive to solve, there are always a few delicate ways to handle it. One of them is to look for a simple solution and expand around it, evaluating this way the dynamical behavior of the system. Stated otherwise, we look in the equations of motion for the simplest solution available by one of 
the variables and, given this solution, we study the fluctuations of the rest of the variables around it. We call such a fluctuation a Normal Variational Equation (NVE).

Taking up the equations of motion (1.17), we easily see that their jet bundle prefers the point

$$
r=\dot{r}=\ddot{r}=\chi=\dot{\chi}=\ddot{\chi}=0,
$$

which satisfies the $r$ and $\chi$ equations, while the one for $\rho$ becomes

$$
\ddot{\rho}=\frac{f_{1}^{\prime}}{2 f_{1}}\left(\dot{\rho}^{2}-E^{2}\right)
$$

yielding the simple solution

$$
\rho_{s o l}=E \tau
$$

where we omit an integration constant without loss of generality. Notice that having all variables - but $\rho$ - vanish is the simplest way to go, the rest of the choices leading to complicated solutions for $r$ or $\chi$.

Since the Virasoro constraint $(1.18)$ is essentially the equation of motion for the worldsheet metric and as such holds independently from the string coordinates' equations of motion, (1.17), it should reflect the same physics, at least classically, if not a more constrained one (since it is of first order). Indeed, enforcing the choice (1.21) onto the Virasoro constraint we acquire

$$
\dot{\rho}^{2}=E^{2}
$$

i.e. the same solution as (1.23). Depending on the particular quality of a system, one can choose to seek for a simple solution on either the standard string equations of motion or on the Virasoro constraint. Regardless, any invariant plane we choose to fluctuate on must be a solution of both the string coordinates' equation of motion and the Virasoro constraint, in order for it to be consistent with our string embedding.

Now, since the simple solution $\rho_{\text {sol }}$ is localized on the point 1.21 , then it is that point around which we study the fluctuations of $r, \chi$. Letting $r(\tau)=0+\epsilon \varrho(\tau)$ into the $r$ equation of motion in (1.17), we expand for $\epsilon \rightarrow 0$ and obtain its NVE at leading order as 


$$
\begin{aligned}
& \ddot{\varrho}(\tau)+\mathcal{B}_{\varrho}(\tau) \dot{\varrho}(\tau)+\mathcal{A}_{\varrho}(\tau) \varrho(\tau)=0, \\
& \mathcal{B}_{\varrho}(\tau)=\left.\frac{E f_{1}^{\prime}}{f_{1}}\right|_{\rho_{\text {sol }}}, \quad \mathcal{A}_{\varrho}(\tau)=\left.\frac{E^{2}+\nu^{2} f_{1}^{2}}{f_{1}^{2}}\right|_{\rho_{\text {sol }}} .
\end{aligned}
$$

In the same manner, letting $\chi(\tau)=0+\epsilon x(\tau)$ we obtain the NVE for $\chi$ as

$$
\begin{gathered}
\ddot{x}(\tau)+\mathcal{B}_{x}(\tau) \dot{x}(\tau)+\mathcal{A}_{x}(\tau) x(\tau)=0, \\
\mathcal{B}_{x}(\tau)=\left.\frac{E f_{2}^{\prime}}{f_{2}}\right|_{\rho_{s o l}}, \quad \mathcal{A}_{x}(\tau)=\left.\frac{\kappa^{2} f_{2}-\kappa E f_{4}^{\prime}}{f_{2}}\right|_{\rho_{s o l}} .
\end{gathered}
$$

Using the change of variable $y=e^{\frac{1}{2} \int \mathcal{B}} z$ in the above differential equations, we deduce two new ones of the kind

$$
z^{\prime \prime}=\mathcal{V} z, \quad \mathcal{V}=\frac{1}{4}\left(2 \mathcal{B}^{\prime}+\mathcal{B}^{2}-4 \mathcal{A}\right)
$$

where $y$ is Liouvillian if and only if $z$ is Liouvillian and, thus, no generality is lost. In this new variable, the NVEs for $r$ and $\chi$ read

$$
\begin{array}{ll}
\ddot{\varrho}=\mathcal{V}_{\varrho} \varrho, & \mathcal{V}_{\varrho}=-\nu^{2}-\frac{E^{2}\left(4+\left(f_{1}^{\prime}\right)^{2}-2 f_{1} f_{1}^{\prime \prime}\right)}{4 f_{1}^{2}}, \\
\ddot{x}=\mathcal{V}_{x} x, & \mathcal{V}_{x}=-\kappa^{2}-\frac{E\left(E\left(f_{2}^{\prime}\right)^{2}-2 f_{2}\left(2 \kappa\left(f_{4}^{\prime}\right)^{2}+E f_{2}^{\prime \prime}\right)\right)}{4 f_{2}^{2}},
\end{array}
$$

Therefore, we end up with two linear, second order, ordinary differential equations. After defining $f_{i}\left(\rho_{\text {sol }}\right)$ in every supergravity class, each $\mathcal{V}$ - which we call the potential - will turn out to be a rational function of $\tau$. Hence, eventually, equations (1.28)- 1.29 for $r$ and $\chi$ are of the appropriate form to be examined by differential Galois theory for Liouvillian integrability.

Differential Galois theory on differential equations boils down to Kovacic's algorithm, [45]. Kovacic provided three criteria on the pole structure of differential equations of the form 
(1.25) and 1.26 that decide if a Liouvillian solution can exist. These conditions are necessary but not sufficient for integrability. In other words, if none of these criteria is satisfied then we deduce with certainty that no Liouvillian solution exists. In that case, the dynamical sector under examination and, thus, the whole theory are non-integrable. On the other hand, even if one of the criteria is satisfied, then such a solution may exist and if it does then Kovacic's algorithm will find it. If the algorithm fails, no Liouvillian solution exists. A detailed analysis is found in Appendix A.

In what follows, we employ the analysis of the present section to examine separately each $\mathrm{AdS}_{3} \times \mathrm{S}^{2} \times \mathbb{R} \times \mathrm{CY}_{2}$ supergravity class of the form [1.1), first presented in 31. After defining each class through the functions $f_{i}(\rho)$ and, thus, specify the corresponding NVEs, we intend to put Kovacic's theorem into action.

\subsection{Class I backgrounds}

Given the general form of the NS-NS sector of the $\mathrm{AdS}_{3} \times \mathrm{S}^{2} \times \mathbb{R} \times \mathrm{CY}_{2}$ massive IIA supergravity, at string frame, as

$$
\begin{gathered}
\mathrm{d} s^{2}=f_{1} \mathrm{~d} s_{\mathrm{AdS}_{3}}^{2}+f_{2} \mathrm{~d} s_{\mathrm{S}^{2}}^{2}+\frac{\mathrm{d} \rho^{2}}{f_{1}}+f_{3} \mathrm{~d} s_{\mathrm{CY}_{2}}^{2}, \\
B_{2}=f_{4} \operatorname{vol}_{\mathrm{S}^{2}}, \quad e^{-\Phi}=f_{5}, \quad f_{i}=f_{i}\left(u, h_{4}, h_{8}\right),
\end{gathered}
$$

then the first supergravity class is defined by the warp factors

$$
\begin{aligned}
& f_{1}=\frac{u}{\sqrt{h_{4} h_{8}}}, \quad f_{2}=f_{1} \frac{h_{4} h_{8}}{4 h_{4} h_{8}+\left(u^{\prime}\right)^{2}}, \quad f_{3}=\sqrt{\frac{h_{4}}{h_{8}}}, \\
& f_{4}=\frac{1}{2}\left(-\rho+\frac{u u^{\prime}}{4 h_{4} h_{8}+\left(u^{\prime}\right)^{2}}\right), \quad f_{5}=\frac{h_{8}^{\frac{3}{4}}}{2 h_{4}^{\frac{1}{4}} \sqrt{u}} \sqrt{4 h_{4} h_{8}+\left(u^{\prime}\right)^{2}} .
\end{aligned}
$$

For simplicity, we treat the functions $h, u$ in a general manner, as in (1.4), i.e.

$$
u(\rho)=c_{3} \rho, \quad h_{4}(\rho)=c_{4}+c_{5} \rho, \quad h_{8}(\rho)=c_{1}+F_{0} \rho,
$$

since their piecewise character, (1.4)-(1.5), can be always assumed. Meaning, whatever result we reach can be assumed to hold for any interval of these functions along the $\rho$ dimension.

Notice that $h_{4}$ and $h_{8}$ can only vanish at the beginning and at the end of the $\rho$ coordinate. Otherwise, the background would degenerate and blow up at points along $\rho$. In fact, both 
of these functions vanish at $\rho=0$ and at least one of them has to vanish on the end of the $\rho$ dimension, $\rho_{f}$, for the space to end in a smooth fashion. This, also, results in a constant dilaton field near and on $\rho=0$. Hence, $h_{4}$ and $h_{8}$ preserve their sign: they begin as positive piecewise linear curves and they remain this way, while their slope decreases along $\rho$. An example is drawn in Figure 1.2

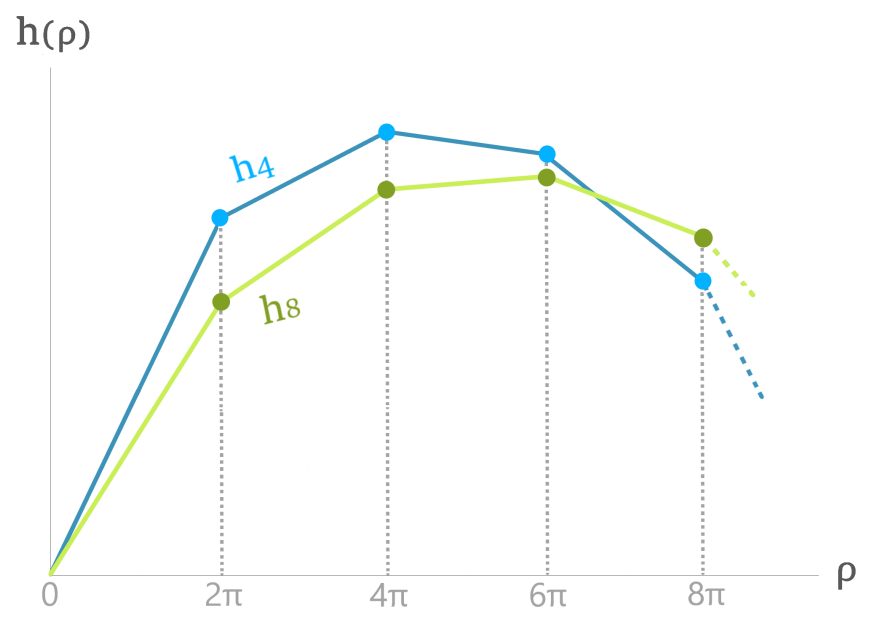

Figure 1.2: An example of the linear functions $h_{4,8}(\rho)$ in Class I backgrounds. These piecewise functions decrease in slope along $\rho$ and at least one of them (or both) has to vanish at the end of the dimension, $\rho_{f}$.

\subsubsection{Abelian T-dual of $\mathrm{AdS}_{3} \times \mathrm{S}^{3} \times \mathrm{T}^{4}$}

Although we chose the functions $u, h_{4}, h_{8}$ such that Class I backgrounds begin and end in a smooth fashion, i.e. (1.32) and Figure 1.2, it is worth breaking that rule for a brief moment. That is, we can trivially choose their most general form (1.4) to reduce to constant functions, i.e. $u=c_{2}, h_{4}=c_{4}$ and $h_{8}=c_{1}$. Then the background reduces to

$$
\begin{aligned}
\mathrm{d} s^{2} & =R^{2}\left(\mathrm{~d} s_{\mathrm{AdS}_{3}}^{2}+\frac{1}{4} \mathrm{~d} s_{\mathrm{S}^{2}}^{2}\right)+\frac{\mathrm{d} \rho^{2}}{R^{2}}+\sqrt{\frac{c_{4}}{c_{1}}} \mathrm{~d} s_{\mathrm{CY}_{2}}^{2}, \\
B_{2} & =-\frac{\rho}{2} \operatorname{vol}_{\mathrm{S}^{2}}, \quad \Phi \sim \text { const. },
\end{aligned}
$$

which is the Abelian T-dual (ATD) of $\mathrm{AdS}_{3} \times \mathrm{S}^{3} \times \mathrm{T}^{4}$. The latter symmetric background is classically integrable, 74. Hence, its Abelian T-dual, this duality being a canonical transformation, will preserve bosonic, classical integrability. This last statement was formally elaborated in [30]. Thus, the trivial choice of constant functions $u, h_{4}, h_{8}$, which is slightly 
outside the smooth choices we consider, leads to an integrable background.

Note, however, that the T-dualized $\mathrm{S}^{1}$, in $\mathrm{S}^{3}$, is contractible. This means that strings oscillating in $\mathrm{S}^{1}$ become massless in the dual vacuum, in the limit of the vanishing radius. Hence, the spectrum should be complemented with those additional massless modes, while the low-energy theory is not supergravity anymore.

Notice, also, that we only picked $\mathrm{CY}_{2}=\mathrm{T}^{4}$, since global metrics on $\mathrm{K} 3$ are not explicitly known. They should exist from Yau's theorem, but this fact is obviously useless wrt examining string dynamics on these surfaces. The same holds, of course, in the next subsection.

\subsubsection{Non-Abelian T-dual of $\mathrm{AdS}_{3} \times \mathrm{S}^{3} \times \mathrm{T}^{4}$}

Before the general treatment, a provoking choice of parameters in 1.32 is $c_{1}=c_{4}=0$, since then $\mathrm{AdS}_{3}$ unwarps from the rest of the space and the background reduces to

$$
\begin{aligned}
\mathrm{d} s^{2} & =R^{2} \mathrm{~d} s_{\mathrm{AdS}_{3}}^{2}+\left(\frac{R^{2} \rho^{2}}{R^{4}+4 \rho^{2}}\right) \mathrm{d} s_{\mathrm{S}^{2}}^{2}+\frac{\mathrm{d} \rho^{2}}{R^{2}}+\sqrt{\frac{c_{5}}{F_{0}}} \mathrm{~d} s_{\mathrm{CY}_{2}}^{2}, \\
B_{2}=\left(-\frac{2 \rho^{3}}{R^{4}+4 \rho^{2}}\right) \operatorname{vol}_{\mathrm{S}^{2}}, & \Phi \sim-\ln \left(1+\rho^{2}\right),
\end{aligned}
$$

where $R^{2}=\frac{c_{3}}{\sqrt{c_{5} F_{0}}}$. This particular background is the non-Abelian T-dual (NATD) of $\mathrm{AdS}_{3} \times \mathrm{S}^{3} \times \mathrm{T}^{4}$, having dualised one of the $\mathrm{SU}(2)$ subgroups of $\mathrm{S}^{3}$, 75. The latter symmetric background is classically integrable, 74. Hence, its non-Abelian T-dual, this duality being a canonical transformation, will preserve bosonic integrability. Therefore, $c_{1}=c_{4}=0$ leads to an integrable background, (1.34), or, more generally, to an integrable interval of this class of backgrounds 6 .

Note that the dilaton field in the vacuum (1.34) ranges from zero, at $\rho=0$, to a negative constant, at $\rho=2 \pi k$, which yields that the string coupling constant has range $g_{s} \in(0,1]$. Hence, the superstring is not strongly coupled on this vacuum and allows for the supergravity approximation.

Now, since this particular choice of parameters gives an integrable structure, this should be reflected on the corresponding $r$ and $\chi$ NVEs. Indeed, this is the case and the details are given in Appendix B.

Recalling that $h_{4}$ and $h_{8}$ are defined piecewise in $\rho,(1.4)-(1.5)$, we realize that the choice $c_{1}=c_{4}=0$ reflects only the first interval, $[0,2 \pi]$, of both the functions. That would be the

\footnotetext{
${ }^{6}$ Letting $c_{1}=c_{4}=0$ be true for all intervals, we inherit an overall NATD integrable theory. Letting it be true for a specific $\rho$-interval means that the background on this particular interval is an integrable NATD of $\mathrm{AdS}_{3} \times \mathrm{S}^{3} \times \mathrm{T}^{4}$. Henceforth, we study all other cases except the trivial one where $c_{1}=c_{4}=0$ everywhere.
} 
first interval for both curves in Figure 1.2. Thus, we conclude that all possible geometries in this supergravity class begin as NATDs of $\mathrm{AdS}_{3} \times \mathrm{S}^{3} \times \mathrm{T}^{4}$ with radius $R^{2}=\frac{c_{3}}{\sqrt{c_{5} F_{0}}}$ and are integrable in that part of their space.

Then $h_{4}$ and $h_{8}$ drive along $\rho$ as positive functions of decreasing slope and, depending on the particular selection of their parameters $\left\{c_{i}, F_{0}\right\}$, they may give various backgrounds associated with appropriate brane set-ups. The positiveness of $h_{4}(\rho)=c_{4}+c_{5} \rho$ and $h_{8}(\rho)=c_{1}+F_{0} \rho$ combined with the decreasing slopes along $\rho$ mean that $c_{1}$ and $c_{4}$ are always non-negative,

$$
c_{1}, c_{4} \geq 0, \quad c_{1} c_{4} \geq 0
$$

while increasing (or staying the same) across the intervals?. This is equivalent to saying that each linear curve on every interval of Figure 1.2 has a non-negative projection on the $\rho=0$ axis. Apart from providing a clearer picture on the overall brane set-up, this statement will define the outcome of the next section where we investigate integrability.

Expanding near $\rho \rightarrow 0^{+}$the space becomes $\mathrm{AdS}_{3} \times \mathbb{R}^{3} \times \mathrm{T}^{4}$, which is symmetric and integrable, as expected for any vicinity of an integrable background like (1.34). Hence, our study of (non-) integrability narrows down to all other intervals except that first NATD one and, from now on, it is those intervals that our study implies.

\subsubsection{NVE for the radial dimension}

Let us begin our integrability analysis on the intervals next to the first NATD one, by first studying the string dynamics along $r$. Letting the warp factors 1.31 roll on the NVE for $r, 1.28$, we obtain

$$
\ddot{\varrho}=\frac{Q_{\mathrm{I}}}{\tau^{2}\left(\tau+\frac{c_{4}}{c_{5} E}\right)^{2}\left(\tau+\frac{c_{1}}{F_{0} E}\right)^{2}} \varrho
$$

where $Q_{\mathrm{I}}=Q_{\mathrm{I}}\left(\tau^{6}, c_{i}, F_{0}, E\right)$ is a long polynomial in the numerator whose explicit form will not concern us. Now, the object that essentially needs to fall under our microscope is the potential $\mathcal{V}_{\varrho}$. Here, it comes with three poles of order two, $\left\{\tau_{1}=0, \tau_{2}=-\frac{c_{4}}{c_{5} E}, \tau_{3}=-\frac{c_{1}}{F_{0} E}\right\}$ and it expands around $\tau \rightarrow \infty$ as

$$
\mathcal{V}_{\varrho}^{\infty}=-\left(\frac{c_{5} F_{0} E^{2}}{c_{3}^{2}}+\nu^{2}\right)-\frac{\left(c_{1} c_{5}+c_{4} F_{0}\right) E}{c_{3} \tau}+\mathcal{O}\left(\frac{1}{\tau^{2}}\right),
$$

\footnotetext{
${ }^{7}$ In case of confusion, $c_{4}$ and $F_{0}$ here represent the constants of $h_{4}$ and $h_{8}$ in a random interval. According to the piecewise definition $1.4-1.5$, these would reflect to the constants $c_{4}^{k}-c_{5}^{k} k$ and $c_{1}^{k}-F_{0}^{k} k$, respectively.
} 
exhibiting zero order behavior there. Thus, $\mathcal{V}_{\varrho}$ satisfies the first and second Kovacic's criteria, implying that the NVE 1.36 may have Liouvillian solutions. However, Kovacic's algorithm fails to solve it as it is.

Nevertheless, the above potential is defined on general parameters whose adjustment equals picking different supergravity backgrounds. Therefore, the failure of Kovacic's algorithm here just states that not all possible backgrounds are integrable. It does not say that there are no integrable ones, among the whole class. This can be also realized by the fact that we have already found, in the previous subsection 1.34, an integrable selection of parameters, i.e. $c_{1}=c_{4}=0$. It's this kind of possible combinations of these parameters (like $c_{1}=c_{4}=0$ ), i.e. particular supergravity backgrounds, that we are interested in, if any (others) exist.

Therefore, we shall utilize the full power of Kovacic's method. This way, if there are any selections of $\left\{c_{i}, F_{0}\right\}$ that allow for Liouvillian solutions of (1.36), we shall find them along with their associated solutions. If such selections are impossible, then we shall safely declare the whole supergravity class as non-integrable.

Kovacic's analytic algorithm is a step-by-step procedure, detailed in Appendix A. Overall, it states that each one of its criteria is associated with a sub-algorithm, called a Case, that may (or may not) solve the equation at hand. As proved above, our NVE (1.36) satisfies the first and second criteria and, thus, must be undertaken by Cases 1 and 2, respectively, of the algorithm.

Since there is nothing intuitive about Kovacic's method, the explicit calculations of the analytic algorithm on all Cases are held in Appendix C. In the main chapter, we just present the results of the algorithm and act with our string-theory considerations on them.

\subsubsection{Case 1}

Case 2 takes into account that Case 1 does not hold, hence we shall always begin by considering Case 1 of Kovacic's theorem. The algorithm for this particular Case is explained in Appendix A.1 and the explicit calculation on our $r$ NVE (1.36) is given in Appendix C.1.

Up to some real constants and signs that we do not care about here, the algorithm produces the quantity

$$
d \sim i \sqrt{\frac{c_{1} c_{4}}{c_{3}^{2}}} \pm \frac{i\left(c_{1} c_{5}+c_{4} F_{0}\right) E}{2 \sqrt{c_{3}^{2} c_{5} F_{0} E^{2}+c_{3}^{4} \nu^{2}}}
$$

and states that $d$ has to be a non-negative integer. If $d$ is such a number, then the algorithm moves on to its next stage. If $d$ is never such a number, then Case 1 cannot give a Liouvillian solution. In other words, integrability demands the above object to be real. 
Therefore, we have reduced our integrability problem to whether there are any interrelations between the supergravity parameters $\left\{c_{i}, F_{0}\right\}$ that let 1.38 to be real. Such a relation would correspond to a specific background. In what follows, we prove that these parameters are constrained by the behavior of the rank functions $h_{4}\left(\rho ; c_{4}, c_{5}\right)$ and $h_{8}\left(\rho ; c_{1}, F_{0}\right)$, in such a way that no such relations can exist.

So, there are three possibilities for 1.38 to be real: either both imaginary terms vanish simultaneously, either they cancel each other out or they both end up real.

The first possibility is excluded since $c_{1}, c_{4} \neq 0$, the opposite being true only on the first $\rho$ interval of the space (the NATD part). Alternatively, if $c_{1}=0$ while $c_{4} \neq 0$ then the first term may vanish but the second one (which also has to vanish) implies $F_{0}=0$, which together lead to $h_{8}=0$. But, as argued repeatedly, $h_{4}, h_{8}=0$ can only happen at the beginning and at the end of the space, otherwise the background degenerates and blows up. The same holds for $c_{1} \neq 0$ while $c_{4}=0$.

The second possibility is also excluded, since the first term is $\nu$-independent and the second $\nu$-dependent. $\nu$ is the string winding number and can be anything, while we want a relation between parameters for all possible string configurations. Notice that this is another good example of why all the available stringy character, in a supergravity (non-) integrability test, is always welcome.

Last but not least, the third possibility is excluded too, since in 1.35 we proved that $c_{1} c_{4} \geq 0$ always and, hence, the first term in 1.38 can never be a positive real number. Since the first term cannot be real nor vanish we don't have to check whether the second term does.

Nevertheless, let us look up the second term of 1.38, for completeness. The second term has a $\nu$-dependent square root, meaning that the root argument cannot be fixed as negative and, thus, cannot produce an $i$ factor in order to end up with a non-zero real number. Therefore, the only possibility left is for this term to vanish. This only happens when

$$
c_{1} c_{5}=-c_{4} F_{0} \quad \Rightarrow \quad c_{1}=-\frac{c_{4} F_{0}}{c_{5}}
$$

which, if we substitute in the first term of $(1.38)$ and demand reality, gives $c_{5} F_{0}>0$. But then, given that $c_{5} F_{0}>0$ together with $c_{1} c_{4}>0$, the initial assumption $c_{1} c_{5}=-c_{4} F_{0}$ can never hold 8 . As expected, we end up with the same result.

One could also argue whether the instantonic mode $E=0$ is an option to vanish the second term in (1.38). The fact is that by choosing $E=0$, we select a particular configuration for our embedding. Even if the $E=0$ mode was integrable it would make no difference, since for $E \neq 0$ the configurations are non-integrable as shown above. While an integrable

\footnotetext{
${ }^{8}$ We can include the possibility that $c_{1} c_{5}+c_{4} F_{0}=0$ when $c_{5}=F_{0}=0$, but then this doesn't stop the first term from being imaginary.
} 
sector of the theory should exhibit its homonymous property on its wholeness, i.e. for all configurations of the string embedding. That is the reason we only look for special selections of $\left\{c_{i}, F_{0}\right\}$, but not of $E, \nu, \kappa$. For the curious mind, the instanton $E=0$ leads here to a non-Liouvillian solution.

Subsequently, $d$ can never be a non-negative integer and, thus, Case 1 cannot provide us a Liouvillian solution. Of course, our NVE (1.36) also satisfies the second Kovacic's criterion and, to that end, we still have a chance to spot integrability through Case 2.

\subsubsection{Case 2}

This Case is explained in Appendix A.2 and the explicit calculation on our $r$ NVE 1.36 is given in Appendix C.2. Here, the algorithm produces the integer quantities $E_{i} \cap \mathbb{Z}$,

$$
E_{1}=\left\{2-4 \sqrt{-\frac{c_{1} c_{4}}{c_{3}^{2}}}, 2,2+4 \sqrt{-\frac{c_{1} c_{4}}{c_{3}^{2}}}\right\}, \quad E_{2}=E_{3}=\{-1,2,5\}
$$

However, as already shown in (1.35) and used on the previous Case, $c_{1} c_{4} \geq 0$. Which means that the quantities under the square roots in $E_{1}$ are non-positive and thus give overall imaginary numbers or 2 . In any case, since $E_{i}$ 's have to be integers, we conclude that $E_{1}=\{2\}$.

Given these $E_{i}$ 's, the algorithm builds a rational function based on the pole structure of $\mathcal{V}_{\varrho}$ as

$$
\theta=\frac{1}{\tau}-\frac{1}{2\left(\tau+\frac{c_{4}}{c_{5} E}\right)}-\frac{1}{2\left(\tau+\frac{c_{1}}{F_{0} E}\right)}
$$

and dictates that the equation

$$
\theta^{\prime \prime}+3 \theta \theta^{\prime}+\theta^{3}-4 \mathcal{V}_{\varrho} \theta-2 \mathcal{V}_{\varrho}^{\prime}=0
$$

must be satisfied, in order for a Liouvillian solution to exist. Replacing $\theta,(1.41)$, into the latter necessary condition we find out that it is not satisfied. Therefore, Case 2 also fails to provide a Liouvillian solution.

Since both Cases failed to expose integrability, we may now declare this class of supergravity backgrounds as non-integrable. Of course, since dynamics along the $r$ dimension is non-integrable we don't have to study the NVE for $\chi$ and our analysis can cease at this point. 
This whole section, dedicated on the $r$ NVE (1.36), was a prototype example of the analytic enforcement of Kovacic's algorithm. Since this differential equation was parametrized by $\left\{c_{1}, . ., c_{5}, F_{0}\right\}$ we employed the algorithm analytically in order to find any special relations between the parameters that would allow for a Liouvillian solution. In our particular case, however, by demanding consistency on those brane-set-up parameters, we proved that no such relations can exist.

The bottom line is that the above procedure is necessary if one wants to study nonintegrability, through differential Galois theory, on a parametrized differential equation. Failure of Kovacic's algorithm without exploring the possible selections between the parameters does not imply the non-integrability of the system. It just states that not all choices of the parameters lead to an integrable system. By which we mean that particular combinations of the parameters may produce Liouvillian solutions. That is, if we are allowed to play with the parameters. If full generality on them is necessary, for any reason, then the analytic application of the algorithm is not needed.

\subsection{Class II backgrounds}

Reminding ourselves for one last time the general form of the NS-NS sector of the $\mathrm{AdS}_{3} \times \mathrm{S}^{2} \times$ $\mathbb{R} \times \mathrm{CY}_{2}$ massive IIA supergravity, at string frame, as

$$
\begin{gathered}
\mathrm{d} s^{2}=f_{1} \mathrm{~d} s_{\mathrm{AdS}_{3}}^{2}+f_{2} \mathrm{~d} s_{\mathrm{S}^{2}}^{2}+\frac{\mathrm{d} \rho^{2}}{f_{1}}+f_{3} \mathrm{~d} s_{\mathrm{CY}_{2}}^{2}, \\
B_{2}=f_{4} \operatorname{vol}_{\mathrm{S}^{2}}, \quad e^{-\Phi}=f_{5}, \quad f_{i}=f_{i}\left(u, h_{4}, h_{8}\right),
\end{gathered}
$$

then the second supergravity class is defined by the warp factors

$$
\begin{aligned}
& f_{1}=\frac{u}{\sqrt{h_{4}^{2}-h_{8}^{2}}}, \quad f_{2}=f_{1} \frac{h_{4}^{2}-h_{8}^{2}}{4\left(h_{4}^{2}-h_{8}^{2}\right)+\left(u^{\prime}\right)^{2}}, \quad f_{3}=\frac{\sqrt{h_{4}^{2}-h_{8}^{2}}}{h_{4}}, \\
& f_{4}=\frac{1}{2}\left(-\rho+\frac{u u^{\prime}}{4\left(h_{4}^{2}-h_{8}^{2}\right)+\left(u^{\prime}\right)^{2}}\right)+\frac{h_{8}}{h_{4}} \hat{J}, \quad f_{5}=\frac{h_{4} \sqrt{4\left(h_{4}^{2}-h_{8}^{2}\right)+\left(u^{\prime}\right)^{2}}}{2 \sqrt{u}\left(h_{4}^{2}-h_{8}^{2}\right)^{\frac{1}{4}}},
\end{aligned}
$$

where $\hat{J}$ is a 2 -form on $\mathrm{CY}_{2}$. For simplicity, again, we treat the functions $h, u$ in a general manner, as in (1.4), i.e. 


$$
u(\rho)=c_{3} \rho, \quad h_{4}(\rho)=c_{4}+c_{5} \rho, \quad h_{8}(\rho)=c_{1}+F_{0} \rho,
$$

since their piecewise character, (1.4)- 1.5 , can be always assumed. Observe that it must be always true that $h_{4} \geq h_{8} \geq 0$.

Notice that, in this supergravity class, the condition for the background to be smooth at the beginning and at the end of the $\rho$ dimension is $\left.h_{4}\right|_{\rho=0}=\left.h_{8}\right|_{\rho=0}=0$ and $\left.h_{4}\right|_{\rho_{f}}=\left.h_{8}\right|_{\rho_{f}}$, respectively. Hence, $h_{4}$ and $h_{8}$ are positive piecewise linear curves that start from $\left.h_{4}\right|_{\rho=0}=$ $\left.h_{8}\right|_{\rho=0}=0$, with $h_{4}>h_{8}$ always, and decrease in slope until they reunite at the end, $\rho_{f}$, as in Figure 1.3 .

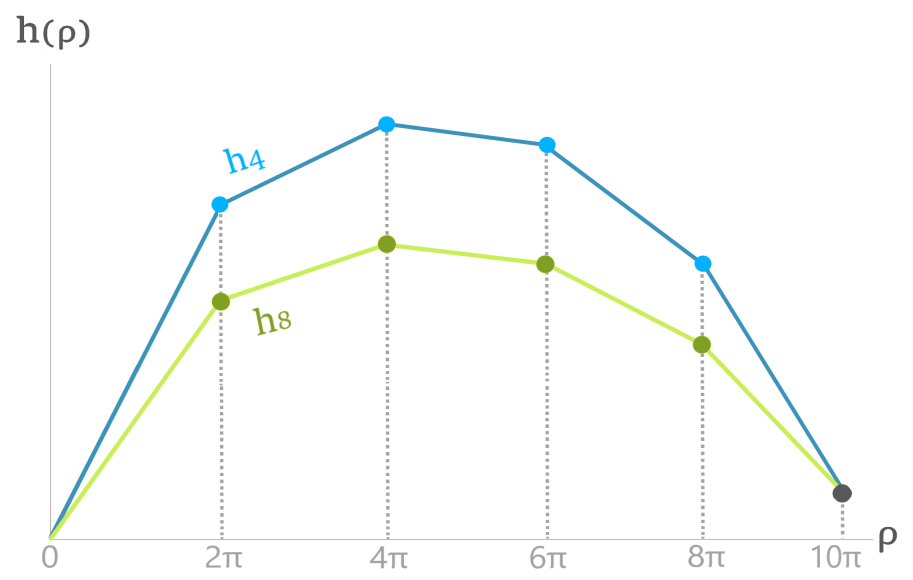

Figure 1.3: An example of the linear functions $h_{4,8}(\rho)$ in Class II supergravity. These piecewise functions start from $\left.h_{4}\right|_{\rho=0}=\left.h_{8}\right|_{\rho=0}=0$, with $h_{4}>h_{8}$ always, and decrease in slope until they reunite at the end, $\rho_{f}=10 \pi$.

\subsubsection{NVE for the radial dimension}

Faithful to the way we treated Class I, let us begin our integrability analysis by first studying the string dynamics along $r$. We again replace the warp factors (1.44) into the NVE for $r$, (1.28), and obtain

$$
\ddot{\varrho}=\frac{Q_{\mathrm{II}}}{\tau^{2}\left(\tau-\frac{c_{1}-c_{4}}{\left(c_{5}-F_{0}\right) E}\right)^{2}\left(\tau+\frac{c_{1}+c_{4}}{\left(c_{5}+F_{0}\right) E}\right)^{2}} \varrho,
$$

where $Q_{\mathrm{II}}=Q_{\mathrm{II}}\left(\tau^{6}, c_{i}, F_{0}, E\right)$ is a long polynomial in the numerator whose explicit form will not concern us. In this class, $\mathcal{V}_{\varrho}$ also comes with three poles of order two, $\left\{\tau_{1}=0, \tau_{2}=\right.$ 
$\left.\frac{c_{1}-c_{4}}{\left(c_{5}-F_{0}\right) E}, \tau_{3}=-\frac{c_{1}+c_{4}}{\left(c_{5}+F_{0}\right) E}\right\}$ and it expands around $\tau \rightarrow \infty$ as

$$
\mathcal{V}_{\varrho}^{\infty}=-\left(\frac{\left(c_{5}^{2}-F_{0}^{2}\right) E^{2}}{c_{3}^{2}}+\nu^{2}\right)-\frac{2\left(c_{4} c_{5}-c_{1} F_{0}\right) E}{c_{3}^{2} \tau}+\mathcal{O}\left(\frac{1}{\tau^{2}}\right)
$$

exhibiting zero order behavior there. Thus, $\mathcal{V}_{\varrho}$ satisfies the first and second Kovacic's criteria, implying that the NVE (1.46) may have Liovillian solutions. However, Kovacic's algorithm fails in this class too to solve it as it is.

Of course, the NVE 1.46$)$ is again parametrized by $\left\{c_{i}, F_{0}\right\}$, whose various inter-relations give different backgrounds in this supergravity class. Therefore, we shall employ for one last time the full power of Kovacic's method to seek out for any such relations that allow for Liouvillian solutions, if any.

Since in this class, the $r$ NVE (1.46) satisfies the first and second Kovacic's criteria too, we will again consider Cases 1 and 2 of Kovacic's theorem.

\subsubsection{Case 1}

As said before, Case 2 takes into account that Case 1 does not hold, thus we again begin by considering Case 1 of Kovacic's theorem. The explicit calculation on our $r$ NVE (1.46) is given in Appendix C.3. Here, up to some real constants and signs, the algorithm produces the quantity

$$
d \sim i \sqrt{\frac{c_{4}^{2}-c_{1}^{2}}{c_{3}^{2}}} \pm \frac{i\left(c_{4} c_{5}-c_{1} F_{0}\right) E}{\sqrt{c_{3}^{2}\left(c_{5}^{2}-F_{0}^{2}\right) E^{2}+c_{3}^{4} \nu^{2}}} .
$$

Again, $d$ has to be a non-negative integer for Case 1 to produce a Liouvillian solution, which in turn means that the above object must be real.

The history repeats itself. There are three possibilities for 1.48) to be real: either both imaginary terms vanish simultaneously, either they cancel each other out or they both end up real. Considering the $\nu$-dependence of the second term, that term can never be a non-zero real number since $\nu$ can be anything for a general string configuration. On the exact same grounds, it can never be canceled against the first term, which is $\nu$-independent. Those arguments exclude the second and third possibility.

The only possibility left is for the second term of 1.48 to vanish, i.e. $c_{4} c_{5}=c_{1} F_{0}$. In turn, the latter condition obligates the first term to give $\left|c_{5}\right| \geq\left|F_{0}\right|$, in the name of reality.

Now, as we argued in the beginning of the section and showed in Figure 1.3, $h_{4}$ and $h_{8}$ are positive piecewise curves that both start from $\left.h_{4}\right|_{\rho=0}=\left.h_{8}\right|_{\rho=0}$ with $h_{4}>h_{8}$ everywhere, and decrease in slope until they reunite at the end, $\left.h_{4}\right|_{\rho_{f}}=\left.h_{8}\right|_{\rho_{f}}$. From simple trigonometry, the fact that $h_{4}$ is always above $h_{8}$ while they both end at the same point $\rho_{f}$ states that: at 
least on the last interval before their reunion, it is true that $c_{4}>c_{1}$. Whatever their slope inter-relation is. Observing Figure 1.3, this statement is equivalent to saying that, on the last interval, $h_{4}$ always has a greater projection on the $\rho=0$ axis than $h_{8}$.

But now, since there has to be at least one region where $c_{4}>c_{1}$, then, combined with the hypothesis $\left|c_{5}\right| \geq\left|F_{0}\right|$, the initial assumption $c_{4} c_{5}=c_{1} F_{0}$ can never hold everywhere.

Therefore, $d$ can never be a non-negative integer and we conclude that Case 1 fails to provide a Liouvillian solution for the second supergravity class. Since $\mathcal{V}_{\varrho}$ satisfies also the second Kovacic's criterion, we move on to examine whether Case 2 can do any better.

\subsubsection{Case 2}

For this last application of Case 2 in Kovacic's theorem, the explicit calculation on our $r$ NVE (1.46) is given in Appendix C.4. Here, the algorithm produces the integer quantities $E_{i} \cap \mathbb{Z}$,

$$
E_{1}=\left\{2-4 \sqrt{\frac{c_{1}^{2}-c_{4}^{2}}{c_{3}^{2}}}, 2,2+4 \sqrt{\frac{c_{1}^{2}-c_{4}^{2}}{c_{3}^{2}}}\right\}, \quad E_{2}=E_{3}=\{-1,2,5\} .
$$

However, as we just showed on the previous subsection, $c_{4}>c_{1}$ at least at the last interval before $h_{4}$ and $h_{8}$ meet at $\rho_{f}$. Thus $c_{1} \geq c_{4}$ can never be always true for any interval, which means that the square root in $E_{1}$ becomes imaginary. Hence, since $E_{i}$ 's have to be integers, we conclude that $E_{1}=\{2\}$.

Since the $E_{i}$ 's are exactly the same with the ones of Class I, the algorithm again builds a rational function, based on the poles of $\mathcal{V}_{\varrho}$ in Class II, as

$$
\theta=\frac{1}{\tau}-\frac{1}{2\left(\tau-\frac{c_{1}-c_{4}}{\left(c_{5}-F_{0}\right) E}\right)}-\frac{1}{2\left(\tau+\frac{c_{1}+c_{4}}{\left(c_{5}+F_{0}\right) E}\right)}
$$

and, the same as the last time, dictates that the equation $\theta^{\prime \prime}+3 \theta \theta^{\prime}+\theta^{3}-4 \mathcal{V}_{\varrho} \theta-2 \mathcal{V}_{\varrho}^{\prime}=0$ should be satisfied. In this class too it does not, therefore Case 2 cannot provide us a Liouvillian solution either, for our $r$ NVE (1.46).

Since both Cases also failed for this class of backgrounds, for any possible selection of the parameters $\left\{c_{i}, F_{0}\right\}$, we declare this supergravity family too as non-integrable. Hence, both supergravity classes are non-integrable and that concludes our integrability adventure on this $\mathrm{AdS}_{3}$ supergravity. 


\subsection{Conclusions}

The apparent conclusion of the present work is the complete, classical, Liouvillian nonintegrability on certain warped backgrounds of the form $\mathrm{AdS}_{3} \times \mathrm{S}^{2} \times \mathbb{R} \times \mathrm{CY}_{2}$, first constructed in [31] and then considered in [33]. Enforcing the full power of Kovacic's theorem, along with simple consistency considerations on the supergravity brane set-ups, we deduced that all possible backgrounds in this warped $\mathrm{AdS}_{3}$ supergravity family are non-integrable.

Note that those considerations were not based on the supergravity approximation of the parameters of the background, which would be an easier but less general way to go. Instead we considered the consistency rules of string theory on Hanany-Witten brane set-ups.

An exception of two integrable choices of backgrounds is when the Class I supergravity solution reduces to the ATD and NATD of $\mathrm{AdS}_{3} \times \mathrm{S}^{3} \times \mathrm{T}^{4}$, for all intervals along the $\rho$ dimension. These unique integrable cases occur when $\mathrm{AdS}_{3}$ unwarps from the rest of the space. Any other warped background for both $\mathrm{AdS}_{3}$ supergravity classes, was proven to be non-integrable.

As a side comment, we note that integrability on AdS supergravity vacua seems to occur only when the AdS part of the space gets unwarped. In the present case, we illustrated that this only happens on Class I, when the background reduces to the integrable ATD and NATD of $\mathrm{AdS}_{3} \times \mathrm{S}^{3} \times \mathrm{T}^{4}$. Then, there is the Sfetsos-Thompson background 62, 75, which is the unwarped integrable case of the Gaiotto-Maldacena $\mathrm{AdS}_{5}$ vacua, [76]. The same also holds for a more recent background [1, among the $\mathrm{AdS}_{7}$ massive IIA supergravity family [38,77]. This argument still holds as just a dominant indication and certainly not as definite statement. However, in [63 and later in [67], it was illustrated that on AdS supergravity vacua that allow for the GKP embedding the AdS space should be unwarped for integrability to occur. This constitutes a strong constraint for many AdS backgrounds, yet it does not apply in our $\mathrm{AdS}_{3}$ family which does not support a GKP vacuum.

Nevertheless, the main aspect of this work is the way we utilize Kovacic's theorem on a differential equation. We illustrated that failure of Kovacic's algorithm on a parametrized equation does not necessarily imply absence of Liouvillian solutions. It just says that there are no such solutions for the full generality of the parameters. If the problem allows to impose any restrictions on its parameters, then a brand new horizon of possibilities appears. On the other hand, if full generality on them is necessary, for any reason, then the analytic application of the algorithm is not needed. In the case when the parameters are adjustable, like with our present supergravity family, then the analytic algorithm must be employed. This way, if there are any selections between the parameters that lead to an integrable result, the algorithm will find them along with the corresponding solutions. Only when this procedure is followed and no such selections are discovered, then we can safely deduce that our system is non-integrable in the Liouvillian sense. 
In our case, the $\mathrm{AdS}_{3}$ supergravity family is defined on general parameters whose adjustment equals picking different supergravity backgrounds. Therefore, the failure of Kovacic's algorithm here just states that not all possible backgrounds are integrable. It does not say that there are no integrable ones, among the whole family. Therefore, we utilized the full power of Kovacic's theorem, by considering its analytic algorithm, and found some necessary conditions - on the background parameters - in order for Liouvillian solutions to exist. By constraining these parameters according to the consistency of the associate brane set-ups, we proved that those necessary conditions can never hold, yielding the complete non-integrability of these vacua. That is, up to the trivial case where the background reduces to the ATD and NATD of $\mathrm{AdS}_{3} \times \mathrm{S}^{3} \times \mathrm{T}^{4}$.

\section{A Differential Galois theory and Kovacic's theorem}

In this appendix we give the basic elements of differential Galois theory that were used by Kovacic [45] to produce his famous algorithm, regarding the existence of Liouvillian solutions on second order linear ordinary differential equations. By a Liouvillian, closed form solution we mean one that is given in terms of algebraic, exponential, trigonometric functions and integrals of those.

The theorem concerns second order linear ordinary differential equations of the form

$$
y^{\prime \prime}(x)+\mathcal{B}(x) y^{\prime}(x)+\mathcal{A}(x) y(x)=0,
$$

where $x \in \mathbb{C}$ and $\mathcal{A}, \mathcal{B}$ are rational complex functions. We can use the variable transformation $y=e^{\frac{1}{2} \int \mathcal{B}} z$ to eliminate the $y^{\prime}$ term and acquire the new equation

$$
z^{\prime \prime}(x)=\mathcal{V}(x) z(x), \quad \mathcal{V}=\frac{1}{4}\left(2 \mathcal{B}^{\prime}+\mathcal{B}^{2}-4 \mathcal{A}\right)
$$

where we shall call $\mathcal{V}$ the potential of the differential equation. Evidently, $y$ exhibits Liouvillian solutions if and only if $z$ does, thus no generality is lost through this change of variable.

The starting point of differential Galois theory on this kind of equations, which is actually Piccard-Vessiot theory, is the group of automorphisms of its solutions, that is $\operatorname{SL}(2, \mathbb{C})$ and its possible subgroups. Letting $G$ be an algebraic subgroup of $\operatorname{SL}(2, \mathbb{C})$, then one of the four cases can occur: 
Case $1 G$ is triangulisable.

Case $2 G$ is conjugate to a subgroup of

$$
\left\{\left(\begin{array}{cc}
c & 0 \\
0 & c^{-1}
\end{array}\right) \mid c \in \mathbb{C}, c \neq 0\right\} \cup\left\{\left(\begin{array}{cc}
0 & c \\
-c^{-1} & 0
\end{array}\right) \mid c \in \mathbb{C}, c \neq 0\right\}
$$

and Case 1 does not hold.

Case $3 G$ is finite and Cases 1 and 2 do not hold.

Case $4 G=S L(2, \mathbb{C})$.

If the differential equation falls into one of the three first cases, it has Liouvillian solutions. On the other hand, if $G=\operatorname{SL}(2, \mathbb{C})$, no such solutions can exist.

The first contribution by Kovacic was to translate Cases 1, 2 and 3 into algebraic arguments on the behavior of $\mathcal{V}$ in 1.52 . These algebraic conditions build up the following theorem.

Theorem 1. The following conditions are necessary for the respective Cases to hold.

Case 1 Every pole of $\mathcal{V}$ must have even order or else have order 1 . The order of $\mathcal{V}$ at $\infty$ must be even or else greater than 2.

Case $2 \mathcal{V}$ must have at least one pole that either has odd order greater than 2 or else has order 2.

Case 3 The order of a pole of $\mathcal{V}$ cannot exceed 2 and the order of $\mathcal{V}$ at $\infty$ must be at least 2.

If $\mathcal{V}=s / t$, then the poles of $\mathcal{V}$ are the zeros of $t$ and the order of the pole is the multiplicity of the zero of $t$. By the order of $\mathcal{V}$ at $\infty$ we shall mean the number $\operatorname{deg} t-\operatorname{deg} s$.

Since these conditions are necessary for the respective cases to hold, then also their failure is sufficient for Case 4 to hold. Therefore we deduce that failure of all three conditions is enough to declare the differential equation (1.52) as non-integrable in the Liouvillian sense.

Nevertheless, if any of the conditions is satisfied, then the respective Case may hold and if it does then a Liovillian solution exists. Hence, when a condition is satisfied we are prompted to the sub-algorithm of the respective Case to examine whether such a solution 
exists and, when it does, use the algorithm to find it. The second contribution by Kovacic was to produce these algorithms for Cases 1, 2 and 3.

\section{A.1 The algorithm for Case 1}

We assume that the necessary condition of Case 1 holds, and we denote by $\Gamma$ the set of poles of $\mathcal{V}$.

Step 1 For each $c \in \Gamma \cup\{\infty\}$ we define a rational function $[\sqrt{\mathcal{V}}]_{c}$ and two complex numbers $\alpha_{c}^{ \pm}$as described below.

$\left(c_{1}\right)$ If $c \in \Gamma$ and $c$ is a pole of order 1 , then

$$
[\sqrt{\mathcal{V}}]_{c}=0, \quad \quad \alpha_{c}^{ \pm}=1
$$

$\left(c_{2}\right)$ If $c \in \Gamma$ and $c$ is a pole of order 2 , then

$$
[\sqrt{\mathcal{V}}]_{c}=0
$$

Let $\beta_{c}$ be the coefficient of $1 /(x-c)^{2}$ in the partial fraction expansion for $\mathcal{V}$. Then

$$
\alpha_{c}^{ \pm}=\frac{1}{2} \pm \frac{1}{2} \sqrt{1+4 \beta_{c}}
$$

$\left(c_{3}\right)$ If $c \in \Gamma$ and $c$ is a pole of order $2 \nu \geq 4$ (necessarily even by the condition for Case 1 ), then $[\sqrt{\mathcal{V}}]_{c}$ is the sum of terms involving $1 /(x-c)^{i}$ for $2 \leq i \leq \nu$ in the Laurent series expansion of $\sqrt{\mathcal{V}}$ at $c$. There are two possibilities for $[\sqrt{\mathcal{V}}]_{c}$, one being the negative of the other, either one may be chosen. Thus

$$
[\sqrt{\mathcal{V}}]_{c}=\frac{a}{(x-c)^{\nu}}+\cdots+\frac{d}{(x-c)^{2}}
$$

Let $\beta_{c}$ be the coefficient of $1 /(x-c)^{\nu+1}$ in $\mathcal{V}$ minus the coefficient of $1 /(x-c)^{\nu+1}$ in $[\sqrt{\mathcal{V}}]_{c}^{2}$. Then

$$
\alpha_{c}^{ \pm}=\frac{1}{2}\left( \pm \frac{\beta_{c}}{a}+\nu\right)
$$

$\left(\infty_{1}\right)$ If the order of $\mathcal{V}$ at $\infty$ is $>2$, then 


$$
[\sqrt{\mathcal{V}}]_{\infty}=0, \quad \alpha_{\infty}^{+}=0, \quad \alpha_{\infty}^{-}=1
$$

$\left(\infty_{2}\right)$ If the order of $\mathcal{V}$ at $\infty$ is 2 , then

$$
[\sqrt{\mathcal{V}}]_{\infty}=0
$$

Let $b_{\infty}$ be the coefficient of $1 / x^{2}$ in the Laurent series expansion of $\mathcal{V}$ at $\infty$. (If $\mathcal{V}=s / t$, where $s, t$ are relatively prime, then $b_{\infty}$ is the leading coefficient of $s$ divided by the leading coefficient of $t$.) Then

$$
\alpha_{\infty}^{ \pm}=\frac{1}{2} \pm \frac{1}{2} \sqrt{1+4 \beta_{\infty}}
$$

$\left(\infty_{3}\right)$ If the order of $\mathcal{V}$ at $\infty$ is $-2 \nu \leq 0$ (necessarily even by the condition of Case 1 ), then $[\sqrt{\mathcal{V}}]_{\infty}$ is the sum of terms involving $x^{i}$ for $0 \leq i \leq \nu$ in the Laurent series for $\sqrt{\mathcal{V}}$ at $\infty$. (Either one of the two possibilities may be chosen.) Thus

$$
[\sqrt{\mathcal{V}}]_{\infty}=a x^{\nu}+\cdots+d
$$

Let $\beta_{\infty}$ be the coefficient of $x^{\nu-1}$ in $\mathcal{V}$ minus the coefficient of $x^{\nu-1}$ in $\left([\sqrt{\mathcal{V}}]_{\infty}\right)^{2}$. Then

$$
\alpha_{\infty}^{ \pm}=\frac{1}{2}\left( \pm \frac{\beta_{\infty}}{a}-\nu\right)
$$

Step 2 For each family $s=(s(c))_{c \in \Gamma \cup\{\infty\}}$, where $s(c)$ is + or - , let

$$
d=\alpha_{\infty}^{s(\infty)}-\sum_{c \in \Gamma} \alpha_{c}^{s(c)}
$$

If $d$ is a non-negative integer, then

$$
\omega=\sum_{c \in \Gamma}\left(s(c)[\sqrt{\mathcal{V}}]_{c}+\frac{\alpha_{c}^{s(c)}}{x-c}\right)+s(\infty)[\sqrt{\mathcal{V}}]_{\infty}
$$

is a candidate for $\omega$. If $d$ is not a non-negative integer, then the family $s$ may be removed from consideration.

Step 3 This step should be applied to each of the families retained from Step 2, until success 
is achieved or the supply of families has been exhausted. In the latter event, Case 1 cannot hold.

For each family, search for a monic polynomial $P$ of degree $d$ (as defined in Step 2) that satisfies the differential equation

$$
P^{\prime \prime}+2 \omega P^{\prime}+\left(\omega^{\prime}+\omega^{2}-\mathcal{V}\right) P=0
$$

This is conveniently done by using undetermined coefficients and is a simple problem in linear algebra, which may or may not have a solution. If such a polynomial exists, then $\eta=P e^{\int \omega}$ is a solution of the differential equation 1.52 . If no such polynomial is found for any family retained from Step 2, then Case 1 cannot hold.

\section{A.2 The algorithm for Case 2}

This algorithm assumes that Case 1 is known to fail. Just as for Case 1, we first collect data for each pole $c$ of $\mathcal{V}$ and also for $\infty$. The form of the data is a set $E_{c}$ (or $E_{\infty}$ ) consisting of from one to three integers. Next we consider families of elements of these sets, perhaps discarding some and retaining others. If no families are retained, Case 2 cannot hold. For each family retained we search for a monic polynomial that satisfies a certain linear differential equation. If no such polynomial exists for any family, then Case 2 cannot hold. If such a polynomial does exist, then a solution to the differential equation 1.52 has been found.

Let $\Gamma$ be the set of poles of $\mathcal{V}$.

Step 1 For each $c \in \Gamma$ we define $E_{c}$ as follows.

$\left(c_{1}\right)$ If $c$ is a pole of order 1 , then $E_{c}=\{4\}$.

$\left(c_{2}\right)$ If $c$ is a pole of order 2 and if $\beta_{c}$ is the coefficient of $1 /(x-c)^{2}$ in the partial fraction expansion of $\mathcal{V}$, then

$$
E_{c}=\left\{2+k \sqrt{1+4 \beta_{c}} \mid k=0, \pm 2\right\} \cap \mathbb{Z}
$$

$\left(c_{3}\right)$ If $c$ is a pole of order $\nu>2$, then $E_{c}=\{\nu\}$.

$\left(\infty_{1}\right)$ If $\mathcal{V}$ has order $>2$ at $\infty$, then $E_{\infty}=\{0,2,4\}$ 
$\left(\infty_{2}\right)$ If $\mathcal{V}$ has order 2 at $\infty$ and $\beta_{\infty}$ is the coefficient of $\mathcal{V}$ in the Laurent series expansion of $\mathcal{V}$ at $\infty$, then

$$
E_{\infty}=\left\{2+k \sqrt{1+4 \beta_{\infty}} \mid k=0, \pm 2\right\} \cap \mathbb{Z}
$$

$\left(\infty_{3}\right)$ If the order of $\mathcal{V}$ at $\infty$ is $\nu<2$, then $E_{\infty}=\{\nu\}$.

Step 2 We consider all families $\left(e_{c}\right)_{c \in \Gamma \cup\{\infty\}}$ with $e_{c} \in E_{c}$. Those families all of whose coordinates are even may be discarded. Let

$$
d=\frac{1}{2}\left(e_{\infty}-\sum_{c \in \Gamma} e_{c}\right)
$$

If $d$ is a non-negative integer, the family should be retained, otherwise the family is discarded. If no families remain under consideration, Case 2 cannot hold.

Step 3 For each family retained from Step 2, we form the rational function

$$
\theta=\frac{1}{2} \sum_{c \in \Gamma} \frac{e_{c}}{x-c} .
$$

Next we search for a monic polynomial $P$ of degree $d$ (as defined in Step 2) such that

$$
P^{\prime \prime \prime}+3 \theta P^{\prime \prime}+\left(3 \theta^{2}+3 \theta^{\prime}-4 \mathcal{V}\right) P^{\prime}+\left(\theta^{\prime \prime}+3 \theta \theta^{\prime}+\theta^{3}-4 \mathcal{V} \theta-2 \mathcal{V}^{\prime}\right) P=0 .
$$

If no such polynomial is found for any family retained from Step 2, then case 2 cannot hold.

Suppose that such a polynomial is found. Let $\varphi=\theta+P^{\prime} / P$ and let $\omega$ be a solution of the equation

$$
\omega^{2}+\varphi \omega+\left(\frac{1}{2} \varphi^{\prime}+\frac{1}{2} \varphi^{2}-\mathcal{V}\right)=0
$$

Then $\eta=e^{\int \omega}$ is a solution of the differential equation 1.52 .

We will not go on to describe the algorithm for Case 3, since we will not be needing it on the present analysis, while it is a bit more of a job than the above Cases 1 and 2. We should note, however, that the necessary algebraic condition that allows for Case 3 to hold is quite restricting and certainly more rare than the others to its satisfaction. If the reader still desires the explicit sub-algorithm for Case 3, Kovacic's original work 45 is the place to visit. 


\section{B NVEs for the non-Abelian T-dual of $\operatorname{AdS}_{3} \times \mathrm{S}^{3} \times \mathrm{T}^{4}$}

Since the particular choice of parameters $c_{1}=c_{4}=0$ gives an integrable structure, this should be reflected on the corresponding $r$ and $\chi$ NVEs. Indeed, replacing this particular choice into the NVE for $r$, 1.28, , the latter becomes

$$
\ddot{\varrho}=-\left(1+\nu^{2}\right) \varrho
$$

which is the harmonic oscillator, integrable as it should. Replacing also into the NVE for $\chi$, (1.29), we acquire

$$
\ddot{x}=\left[-4 \kappa\left(\kappa+\frac{2 E}{R^{2}}\right)-\frac{48 R^{4} E^{2}}{\left(R^{4}+4 E^{2} \tau^{2}\right)^{2}}-\frac{16 R^{2} E \kappa}{R^{4}+4 E^{2} \tau^{2}}\right] x .
$$

This equation satisfies the first and second Kovacic's criteria, but yet the algorithm fails to solve it. However, this not yet the correctly informed NVE. That is, since $c_{1}=c_{4}=0$ reduce the AdS warp factor to a constant, $f_{1}=\frac{c_{3}}{\sqrt{c_{5} F_{0}}}=R^{2}$, then the $t$ equation of motion in 1.17 is solved 9 for the static gaug $e^{10} t=\tau$ and gives $E=R^{2}$ near $r=0$ (around which we fluctuate). Replacing this into 1.55 , we get

$$
\ddot{x}=\left(\frac{-48-4 \kappa\left(1+4 \tau^{2}\right)\left(6+\kappa+4(2+\kappa) \tau^{2}\right)}{\left(1+4 \tau^{2}\right)^{2}}\right) x,
$$

which is now solved by the algorithm 11 , as it should. Note that the above equation is solved for any choice of gauge $t=\lambda \tau, \lambda \in \mathbb{C}$ (and thus every energy $E=\lambda R^{2}$ ), as it is appropriate for equivalent physics. Also, notice that we did not really pick a value for the energy $E-$ the energy depends on the observer, i.e. the choice of gauge - the background picked it by itself and we just informed the system about it.

A special case for the above gauge choice is to set $\lambda=0$, i.e. choose a configuration $t=$ const. . Since the worldsheet theory localizes on target space time $t$, this is an instantonic mode of energy $E=0$. Being one of the legitimate configurations of our string embedding in an integrable space, this instanton has to be integrable as well. Indeed, set-

${ }^{9}$ Equivalently, we can find the energy from the worldsheet conjugate momentum as

$$
E=p_{0}=\int_{0}^{2 \pi} \mathrm{d} \sigma \mathcal{P}_{0}^{\tau}=-\frac{2 \pi}{4 \pi \alpha^{\prime}} 2 g_{00} \dot{t} \stackrel{\alpha^{\prime}=1}{=} \cosh ^{2} r f_{1} \dot{t} \underset{t=\tau}{\stackrel{r=0}{\longrightarrow}} R^{2}
$$

\footnotetext{
${ }^{10}$ This is a privilege of the current situation, where $\left.g_{00}\right|_{r=0}=-R^{2}=$ const. . When $\left.g_{00}(\tau)\right|_{r=0} \neq$ const. , then $t$ behaves as $t=\int \frac{E \mathrm{~d} \tau}{\left.g_{00}(\tau)\right|_{r=0}}$ and thus $E$ cannot be specified as a constant and must remain as it is in the equation.

${ }^{11}$ We omit the solution since it is of substantial size. The curious reader can put the equation in any algebra software to acquire the solution.
} 
ting $E=0$ in the NATD NVE 1.55 we obtain an harmonic oscillator, integrable as it should.

\section{Kovacic's theorem on NVEs}

In this appendix we apply the algorithm presented in Appendix A, to study the $r$ NVE for both supergravity classes. The main body of the chapter was reserved for the essential string theory considerations that exclude integrability. Here we just present the explicit calculations that lead to the necessary conditions on which those considerations act.

\section{C.1 Case 1 for Class I}

First in line is the supergravity Class I, with the $r$ NVE 1.36. We begin by writing the partial fraction expansion of $\mathcal{V}_{\varrho}$ as

$$
\mathcal{V}_{\varrho}=-\left(\frac{c_{5} F_{0} E^{2}}{c_{3}^{2}}+\nu^{2}\right)+\left(\frac{-c_{3}^{2}-4 c_{1} c_{4}}{4 c_{3}^{2}}\right) \frac{1}{\tau^{2}}+\frac{5 / 16}{\left(\tau+\frac{c_{4}}{c_{5} E}\right)^{2}}+\frac{5 / 16}{\left(\tau+\frac{c_{1}}{F_{0} E}\right)^{2}}+\ldots
$$

where the coefficients $\beta_{i}$ of the pole terms $1 /\left(\tau-\tau_{i}\right)^{2}$ are used to construct the complex numbers $\alpha_{i}^{ \pm}=\frac{1}{2} \pm \frac{1}{2} \sqrt{1+4 \beta_{i}}$. In our case these become

$$
\alpha_{1}^{ \pm}=\frac{1}{2} \pm \sqrt{-\frac{c_{1} c_{4}}{c_{3}^{2}}} \quad \alpha_{2}^{ \pm}=\alpha_{3}^{ \pm}=\left\{\begin{array}{c}
\frac{5}{4} \\
-\frac{1}{4}
\end{array}\right.
$$

Next, we move to the $\tau \rightarrow \infty$ regime and define a rational function $\left[\sqrt{\mathcal{V}}_{\varrho}\right]_{\infty}$ which here, since $\mathcal{V}_{\varrho}^{\infty}$ is of zeroth order, it has to be just a complex number, i.e. $\left[\sqrt{\mathcal{V}_{\varrho}}\right]_{\infty}=a$. Then $a$ is found by matching terms between $\left[\sqrt{\mathcal{V}_{\varrho}}\right]_{\infty}^{2}$ and $\mathcal{V}_{\varrho}^{\infty}$ in 1.37 , taking the value $a=i \sqrt{\frac{c_{5} F_{0} E^{2}}{c_{3}^{2}}+\nu^{2}}$. As before, letting $\beta_{\infty}$ be the coefficient of $1 / \tau$ in $\mathcal{V}_{\varrho}^{\infty}$, we construct the complex numbers $\alpha_{\infty}^{ \pm}=\frac{ \pm \beta_{\infty}}{2 a}$ which are now valued

$$
\alpha_{\infty}^{ \pm}= \pm \frac{i\left(c_{1} c_{5}+c_{4} F_{0}\right) E}{2 \sqrt{c_{3}^{2} c_{5} F_{0} E^{2}+c_{3}^{4} \nu^{2}}}
$$

Stepping forward, we gather all our findings $\alpha_{i}^{ \pm}, \alpha_{\infty}^{ \pm}$and, letting $s(\cdot)$ be the sign function, we define the numbers $d=\alpha_{\infty}^{s(\infty)}-\sum_{i} \alpha_{i}^{s(i)}$. Considering all the possible sign combinations, these are $2^{4}=16$ complex numbers. Up to some real constants and signs between their terms, these sixteen numbers are all of the form 12

\footnotetext{
${ }^{12}$ We write $\sqrt{-c_{1} c_{4}} \rightarrow i \sqrt{c_{1} c_{4}}$ for convenience in our following considerations.
} 


$$
d \sim i \sqrt{\frac{c_{1} c_{4}}{c_{3}^{2}}} \pm \frac{i\left(c_{1} c_{5}+c_{4} F_{0}\right) E}{2 \sqrt{c_{3}^{2} c_{5} F_{0} E^{2}+c_{3}^{4} \nu^{2}}}
$$

Kovacic states that $d$ has to be a non-negative integer in order for the algorithm to move on to its next stage. If $d$ is never such a number, then Case 1 cannot give a Liouvillian solution. In other words, the above two terms must be real.

Under the string theory considerations on subsection 1.4.4, we conclude that this can never be the case and, thus, Case 1 cannot hold.

\section{C.2 Case 2 for Class I}

In this Case, we begin by considering the same pole coefficients $\beta_{i}$ that made up the $\alpha_{i}^{ \pm}$ numbers, 1.59. But now $\beta_{i}$ 's construct the coordinates $E_{i}=\left\{2+k \sqrt{1+4 \beta_{i}} \mid k=0, \pm 2\right\} \cap \mathbb{Z}$, which in this case read

$$
E_{1}=\left\{2-4 \sqrt{-\frac{c_{1} c_{4}}{c_{3}^{2}}}, 2,2+4 \sqrt{-\frac{c_{1} c_{4}}{c_{3}^{2}}}\right\} \quad E_{2}=E_{3}=\{-1,2,5\}
$$

Under the string theory considerations on subsection 1.4 .5 we conclude that $E_{1}=\{2\}$.

Next, since our potential at infinity, $\mathcal{V}_{\varrho}^{\infty}$, is of zeroth order, we also define the coordinate $E_{\infty}=\{0\}$. Then, in analogy with Case 1, we gather the coordinates $E_{\infty}, E_{i}$ and define the numbers $d=\frac{1}{2}\left(e_{\infty}-\sum_{i} e_{i}\right)$, where $e_{i} \in E_{i}$ are the particular coordinates. Again, $d$ 's have to be non-integers to be acceptable. Considering all the possible coordinate combinations we calculate $3^{2}=9$ numbers, of which only one is non-negative, i.e. the one for $e_{2}=e_{3}=-1$ ( $e_{\infty}=0$ and $e_{1}=2$ always $)$ that gives $d=0$.

Now, since in this Case we actually obtained a single non-integer $d, d=0$, we may move to the next step. That consists of forming the rational function $\theta=\frac{1}{2} \sum_{i} \frac{e_{i}}{\tau-\tau_{i}}$, in which we use the particular $e_{i}$ 's that made up $d=0$, i.e. $e_{1}=2, e_{2}=e_{3}=-1$. In our case, $\theta$ is

$$
\theta=\frac{1}{\tau}-\frac{1}{2\left(\tau+\frac{c_{4}}{c_{5} E}\right)}-\frac{1}{2\left(\tau+\frac{c_{1}}{F_{0} E}\right)} .
$$

Next we search for a monic polynomial $P$ of degree $d$ such that

$$
P^{\prime \prime \prime}+3 \theta P^{\prime \prime}+\left(3 \theta^{2}+3 \theta^{\prime}-4 \mathcal{V}_{\varrho}\right) P^{\prime}+\left(\theta^{\prime \prime}+3 \theta \theta^{\prime}+\theta^{3}-4 \mathcal{V}_{\varrho} \theta-2 \mathcal{V}_{\varrho}^{\prime}\right) P=0
$$


Since $d=0$ is our only heritage from the previous step, that means $P=1$ and the question reduces to whether $\theta^{\prime \prime}+3 \theta \theta^{\prime}+\theta^{3}-4 \mathcal{V}_{\varrho} \theta-2 \mathcal{V}_{\varrho}^{\prime}=0$. Replacing $\theta$, 1.63 , into the latter necessary condition we find out that it is not satisfied. Therefore, Case 2 also fails to provide a Liouvillian solution.

\section{C.3 Case 1 for Class II}

We begin by writing the partial fraction expansion of $\mathcal{V}_{\varrho}$ as

$\mathcal{V}_{\varrho}=-\left(\frac{\left(c_{5}^{2}-F_{0}^{2}\right) E^{2}}{c_{3}^{2}}+\nu^{2}\right)+\left(\frac{4\left(c_{1}^{2}-c_{4}^{2}\right)-c_{3}^{2}}{4 c_{3}^{2}}\right) \frac{1}{\tau^{2}}+\frac{5 / 16}{\left(\tau-\frac{c_{1}-c_{4}}{\left(c_{5}-F_{0}\right) E}\right)^{2}}+\frac{5 / 16}{\left(\tau+\frac{c_{1}+c_{4}}{\left(c_{5}+F_{0}\right) E}\right)^{2}}+\ldots$

where the coefficients $\beta_{i}$ of the pole terms $1 /\left(\tau-\tau_{i}\right)^{2}$ are used to construct the complex numbers $\alpha_{i}^{ \pm}=\frac{1}{2} \pm \frac{1}{2} \sqrt{1+4 \beta_{i}}$. Here, these become

$$
\alpha_{1}^{ \pm}=\frac{1}{2} \pm \sqrt{\frac{c_{1}^{2}-c_{4}^{2}}{c_{3}^{2}}}, \quad \alpha_{2}^{ \pm}=\alpha_{3}^{ \pm}=\left\{\begin{array}{c}
\frac{5}{4} \\
-\frac{1}{4}
\end{array} .\right.
$$

Next, we move to the $\tau \rightarrow \infty$ regime and define the rational function $\left[\sqrt{\mathcal{V}_{\varrho}}\right]_{\infty}$ which here, since $\mathcal{V}_{\varrho}^{\infty}$ is of zeroth order, it has to be just a complex number, i.e. $\left[\sqrt{\mathcal{V}_{\varrho}}\right]_{\infty}=a$. Then $a$ is found by matching terms between $\left[\sqrt{\mathcal{V}}_{\varrho}\right]_{\infty}^{2}$ and $\mathcal{V}_{\varrho}^{\infty}$ in 1.47 , taking the value $a=$ $i \sqrt{\frac{\left(c_{5}^{2}-F_{0}^{2}\right) E^{2}}{c_{3}^{2}}+\nu^{2}}$. As before, letting $\beta_{\infty}$ be the coefficient of $1 / \tau$ in $\mathcal{V}_{\varrho}^{\infty}$, we construct the complex numbers $\alpha_{\infty}^{ \pm}=\frac{ \pm \beta_{\infty}}{2 a}$ which are now valued

$$
\alpha_{\infty}^{ \pm}= \pm \frac{i\left(c_{4} c_{5}-c_{1} F_{0}\right) E}{\sqrt{c_{3}^{2}\left(c_{5}^{2}-F_{0}^{2}\right) E^{2}+c_{3}^{4} \nu^{2}}} .
$$

We gather all our findings $\alpha_{i}^{ \pm}, \alpha_{\infty}^{ \pm}$and, letting $s(\cdot)$ be the sign function, we define the numbers $d=\alpha_{\infty}^{s(\infty)}-\sum_{i} \alpha_{i}^{s(i)}$. Considering all the possible sign combinations, these are $2^{4}=16$ complex numbers. Up to some real constants and signs between their terms, these sixteen numbers are all of the form 13

$$
d \sim i \sqrt{\frac{c_{4}^{2}-c_{1}^{2}}{c_{3}^{2}}} \pm \frac{i\left(c_{4} c_{5}-c_{1} F_{0}\right) E}{\sqrt{c_{3}^{2}\left(c_{5}^{2}-F_{0}^{2}\right) E^{2}+c_{3}^{4} \nu^{2}}}
$$

\footnotetext{
${ }^{13}$ We write $\sqrt{c_{1}^{2}-c_{4}^{2}} \rightarrow i \sqrt{c_{4}^{2}-c_{1}^{2}}$ for convenience in our following considerations.
} 
Again, $d$ has to be a non-negative integer for Case 1 to produce a Liouvillian solution, which in turn means that the above two terms must be real.

Under the string theory considerations on subsection 1.5.2, we conclude that this can never be the case and, thus, Case 1 cannot hold.

\section{C.4 Case 2 for Class II}

In Case 2, we begin by considering the same pole coefficients $\beta_{i}$ that made up the $\alpha_{i}^{ \pm}$numbers, (1.66). But now $\beta_{i}$ 's construct the coordinates $E_{i}=\left\{2+k \sqrt{1+4 \beta_{i}} \mid k=0, \pm 2\right\} \cap \mathbb{Z}$, which in this case read

$$
E_{1}=\left\{2-4 \sqrt{\frac{c_{1}^{2}-c_{4}^{2}}{c_{3}^{2}}}, 2,2+4 \sqrt{\frac{c_{1}^{2}-c_{4}^{2}}{c_{3}^{2}}}\right\}, \quad E_{2}=E_{3}=\{-1,2,5\}
$$

Under the string theory considerations on subsection 1.5 .3 , we conclude that $E_{1}=\{2\}$.

Since the $E_{i}$ 's are exactly the same with the ones of Class I, we again have a single non-negative integer $d=0$ made out of them, while the rational function $\theta=\frac{1}{2} \sum_{i} \frac{e_{i}}{\tau-\tau_{i}}$ now reads

$$
\theta=\frac{1}{\tau}-\frac{1}{2\left(\tau-\frac{c_{1}-c_{4}}{\left(c_{5}-F_{0}\right) E}\right)}-\frac{1}{2\left(\tau+\frac{c_{1}+c_{4}}{\left(c_{5}+F_{0}\right) E}\right)}
$$

The same as the last time, $\theta$ should satisfy $\theta^{\prime \prime}+3 \theta \theta^{\prime}+\theta^{3}-4 \mathcal{V}_{\varrho} \theta-2 \mathcal{V}_{\varrho}^{\prime}=0$. In this class too it does not, therefore Case 2 cannot provide us a Liouvillian solution either, for our $r$ NVE 1.46. 




\section{Chapter 2}

\section{Non-integrability of the $\Omega$ deformation}

\section{Introduction}

A particular supergravity vacuum that deserves the attention of non-integrability methods was recently discovered in [35]. Neglecting an unimportant warp factor, this background is the holographic dual of the four-dimensional, $\Omega$-deformed $\mathcal{N}=4$ SYM theory. In the same vein of the supergravity realization of the $\Omega$-deformation, a similar study was also recently performed in [78]. $\Omega$-deformation was originally introduced in 79 as a method of calculating the path integral of four-dimensional $\mathcal{N}=2$ gauge theories, through supersymmetric localization. Since then, the deformation and its associated Nekrasov partition function, have produced numerous exact results on supersymmetric quantum field theories on curved manifolds, as well as having laid the foundations for both the Nekrasov-Shatashvili [80] and the AGT [81] correspondences. The background we consider is a deformation of $\mathrm{AdS}_{5} \times \mathrm{S}^{5}$ in type IIB theory that preserves 16 supercharges, while the $\Omega$-deformation manifests itself in this dual gravity as turning on a Kalb-Ramond field (and a $C_{2} \mathrm{RR}$ form). Interestingly, the associated $H_{3}$ flux inter-binds the whole geometry and breaks part of the bosonic symmetries of $\mathrm{AdS}_{5} \times \mathrm{S}^{5}$, both being facts that make this background intractable to classic integrability methods.

The study of non-integrability on this particular background is of interest, since there are significant suggestions linking integrable structures and the $\Omega$-deformation in the present literature. In particular, a connection has been established between the $\Omega$-deformed $\mathcal{N}=2$ gauge theory and quantum integrable Hamiltonian systems, see [80 83] or the more recent [84. Similar work has been done, 85, 86], on a string theory realization [87, 89] of the $\Omega$-deformation, where the resulting models were associated with the TsT subclass of the Yang-Baxter deformation. Considering all these integrable aspects of the $\Omega$-deformation, an indication of non-integrability would consequently suggest an interesting antithesis, worthy of further study.

In this chapter, after a complete symmetry analysis on the ten-dimensional $\Omega$-deformed supergravity background, we accordingly construct string embeddings that are dynamical on 
the asymmetric directions. We do so, in order to have a better chance to spot non-integrable behavior. We then find simple solutions on the equations of motion and let the string fluctuate around them, along each dimension. As it turns out, in each case, one of the fluctuations exhibits a non-Liouvillian solution in terms of the Bessel function of the first kind, yielding the classical non-integrability of our embedding and, therefore, of the whole vacuum under consideration.

\section{The supergravity solution}

The supergravity vacuum dual to the $\Omega$-deformation of $\mathcal{N}=4$ SYM at the conformal point was introduced in 35 . Neglecting a warp factor ${ }^{1}$ which we can set, along with the radii of the space, to unity we obtain the background fields

$$
\begin{aligned}
\mathrm{d} s^{2} & =\frac{\mathrm{d} \vec{x}_{4}^{2}+\mathrm{d} z^{2}}{z^{2}}+\mathrm{d} \theta^{2}-\sin ^{2} \theta \mathrm{d} \phi^{2}+\cos ^{2} \theta \mathrm{d} \Omega_{3}^{2}, \\
B_{2} & =i g_{s} C_{2}=-\frac{\beta e^{-\phi} \sin \theta}{4 z}\left(\mathrm{~d} x_{1} \wedge \mathrm{d} x_{2}+\mathrm{d} x_{3} \wedge \mathrm{d} x_{4}\right), \\
F_{5} & =-\frac{i}{g_{s}}\left(1+\star_{10}\right) \mathrm{d}\left(\frac{1}{z^{4}}\right) \wedge \mathrm{vol}_{4}, \quad e^{\Phi}=g_{s},
\end{aligned}
$$

where $g_{s}$ is the string coupling and vol $_{4}$ the volume of the $\mathbb{R}^{4}$ subspace. $\beta \in \mathbb{R}^{+}$is the deformation parameter in the dual field theory, which was identified with the linear combination $\epsilon_{1}+\epsilon_{2}$ in 90,91$]$. Thus, the $\Omega$-deformation manifests itself as turning on a Kalb-Ramond field (and a $C_{2} \mathrm{RR}$ field) on the integrable $\mathrm{H}_{5} \times \mathrm{dS}_{5}$.

Since the internal space of the IIB background (2.1) is a deformation of the five-dimensional de Sitter space, this implies that the background is actually a solution of type IIB* supergravity 92,93 . Continuing as $\phi \rightarrow i \varphi$, we obtain the Euclidean $\operatorname{AdS}_{5} \times \mathrm{S}^{5}$. The vacuum preserves 16 supercharges and it is the supergravity dual of $\mathcal{N}=4 \mathrm{SYM}$. Interestingly, the non-trivial $H_{3}$ flux inter-binds the geometric subspaces and breaks part of the bosonic symmetries of $\mathrm{AdS}_{5} \times \mathrm{S}^{5}$, both facts that make the background intractable to classic integrability methods.

While the geometry in (2.1) looks like a peculiar continuation of $\operatorname{AdS}_{5} \times S^{5}$, on which the string dynamics could be qualitatively questioned, it is not quite unfamiliar. In fact, it was obtained in 94 by a double Wick rotation on $\mathrm{AdS}_{5} \times \mathrm{S}^{5}$ (in our notation wrt to the $\mathbb{R}^{4}$ time $t \equiv x_{1}$ and $\phi$ ), as a natural formulation on which the holographic principle - for the

\footnotetext{
${ }^{1}$ This is equivalent to setting $w=0$ in 35 . This parametrizes a VEV of a scalar field in a representation of the $\mathrm{SO}(6)$ of $\mathcal{N}=4 \mathrm{SYM}$. In the dual gravity, it generates a distribution of smeared D3-branes.
} 
Penrose limit - naturally associates the bulk with the boundary. In particular, it was shown that, for the BMN string on this geometry, the bulk-to-boundary trajectories are interpreted as a tunneling phenomenon and thus that the BMN boundary-to-boundary correlations are holographically well-defined.

\section{Symmetries of the vacuum}

Since the $\Omega$-deformation is realized on the background as a $B_{2}$-field - that obviously does not respect part of the geometric isometries - it is instructive to perform a symmetry analysis on its associated $H_{3}$ flux. Noting that the geometry (2.1) is a product space and thus its Killing vectors (KVs) are decoupled for the two subspaces, we may vary $H_{3}$ separately along $H^{5}$ and $\mathrm{dS}_{5}$. Here, we present the symmetries of the $\Omega$ vacuum, while the details to extract them are held in Appendix $\mathrm{D}$.

Hence, if $K$ is a $\mathrm{KV}$ on $\mathrm{H}_{5}$, then the vanishing of the Lie derivative $\mathcal{L}_{K} H_{3}=0$ is solved for the vectors

$$
\begin{aligned}
& K_{R_{12}}=x_{1} \partial_{2}-x_{2} \partial_{1}, \\
& K_{R_{34}}=x_{3} \partial_{4}-x_{4} \partial_{3}, \\
& K_{S C_{i}}=\partial_{i}, \quad i=1, \ldots, 4,
\end{aligned}
$$

namely two $\mathrm{SO}(4)$ rotations on $\mathbb{R}^{4}$ and the four $\mathrm{SO}(1,1)$ special conformal Killing vectors ${ }^{2}$ ( $\mathrm{SCKVs}$ ) on $\mathrm{H}_{5}$. As far as the $\mathrm{KVs}_{\mathrm{s}}$ of $\mathrm{dS}_{5}$ are concerned, the only non trivial $\mathrm{KV}$ that leaves $\mathrm{H}_{3}$ invariant is

$$
K_{B}=e^{-\phi}\left(\cot \theta \cos \omega_{1} \partial_{\phi}+\cos \omega_{1} \partial_{\theta}+\tan \theta \sin \omega_{1} \partial_{\omega_{1}}\right)
$$

where $\omega_{1}$ is an angle in $\Omega_{3}$ of $\mathrm{dS}_{5}{ }^{3}$. This rotation is identified as an $\mathrm{SO}(1,1)$ boost of the $\mathrm{SO}(1,5)$ isometry. The rest of the $\mathrm{KVs}$ of $\mathrm{dS}_{5}$ that preserve $H_{3}$ are trivial, namely the six $\mathrm{SO}(4)$ rotations of $\Omega_{3}$ inside $\mathrm{dS}_{5}$.

Note that the symmetry analysis on the background 2.1 is of twofold interest. First, it reveals the action of the $\Omega$-deformation on the symmetry structure of the dual supergravity. Most importantly for our non-integrability method, though, it serves as a beacon on how to push our bosonic string towards a less symmetric embedding, the latter having a better

\footnotetext{
${ }^{2} K=\partial_{i}, i=1, \ldots, 4$, are translations on $\mathbb{R}^{4}$ and special conformal transformations on $\mathrm{H}^{5}$.

${ }^{3}$ There are two more, one for each angle $\omega_{2}, \omega_{3}$ in the 3 -sphere, $\mathrm{d} \Omega_{3}^{2}=\mathrm{d} \omega_{1}^{2}+\sin ^{2} \omega_{1} \mathrm{~d} \Omega_{2}^{2}$, of $\mathrm{d} S_{5}$. It doesn't make any difference in this problem.
} 
chance to exhibit non-integrable dynamics.

\section{String dynamics}

\subsection{The first embedding}

The bosonic string dynamics emerges from the non-linear $\sigma$-model, in conformal gauge,

$$
S_{P}=-\frac{1}{4 \pi \alpha^{\prime}} \int_{\Sigma} \mathrm{d}^{2} \sigma \partial_{a} X^{\mu} \partial_{b} X^{\nu}\left(g_{\mu \nu} \eta^{a b}+B_{\mu \nu} \epsilon^{a b}\right)
$$

where the string coordinates' $X^{\mu}(\tau, \sigma)$ equation of motion is supplemented by the Virasoro constraint $T_{a b}=0$, where the worldsheet energy-momentum tensor is given by

$$
T_{a b}=\frac{1}{\alpha^{\prime}}\left(\partial_{a} X^{\mu} \partial_{b} X^{\nu} g_{\mu \nu}-\frac{1}{2} \eta_{a b} \eta^{c d} \partial_{c} X^{\mu} \partial_{d} X^{\nu} g_{\mu \nu}\right)
$$

with $\tau, \sigma$ being the worldsheet coordinates. Having differential Galois theory in mind, we desire a string embedding that produces second order, ordinary linear differential equations of motion. This means that the string coordinates must be $X^{\mu}=X^{\mu}(\tau)$ or $X^{\mu}=X^{\mu}(\sigma)$. For a closed string in type II theory, this translates into wrapping the string around compact coordinates.

Since $\mathrm{H}_{5} \times \mathrm{dS}_{5}$ is integrable, our chance to spot non-integrable behavior lies along the $H_{3}$ flux. Hence, most importantly, our embedding should incorporate dynamics along the $H_{3}$ flux. The $B_{2}$ field component(s) $B_{x_{1} x_{2}}$ (and $B_{x_{3} x_{4}}$ ) is non-vanishing on the $\sigma$-model (3.32) only for the choice - in these coordinates $-x_{1}=x_{1}(\tau)$ and $x_{2}=x_{2}(\sigma)$, or vice versa. However, such a $\sigma$-dependence produces partial differential equations of motion for a closed string and, thus, it must be excluded.

The resolution comes by changing our coordinates on the $\mathbb{R}^{4}$ subspace of $H_{5}$, from Cartesian to spherical, as

$$
\mathrm{d} \vec{x}_{4}^{2}=d r^{2}+r^{2}\left(\mathrm{~d} \psi^{2}+\sin ^{2} \psi \mathrm{d} \chi^{2}+\sin ^{2} \psi \sin ^{2} \chi \mathrm{d} \xi^{2}\right)
$$

with the old coordinates depending on the new ones as 


$$
\begin{aligned}
& x_{1}=r \cos \psi, \\
& x_{2}=r \sin \psi \cos \chi, \\
& x_{3}=r \sin \psi \sin \chi \cos \xi, \\
& x_{4}=r \sin \psi \sin \chi \sin \xi,
\end{aligned}
$$

In this $\mathbb{R}^{4}$ subspace, we can choose the embedding $r=r(\tau), \chi=\chi(\tau), \xi=\kappa \sigma$, and $\psi=\pi / 2$. Since $H_{3}$ is invariant under only two out of the six $\mathrm{SO}(4)$ rotations of $\mathbb{R}^{4}$, we set $\psi=\pi / 2$ but we leave $\chi=\chi(\tau)$ in order to have some portion of $\mathbb{R}^{4}$ rotations that can bring the equations of motion to the test. The same symmetry analysis also showed that $z$ is non-trivially involved in $H_{3}$ and thus we let $z=z(\tau)$.

As far as $\mathrm{dS}_{5}$ is concerned, we choose $\theta=\theta(\tau)$ and $\phi=\phi(\tau)$ which also parametrize $H_{3}$ non-trivially. The $\Omega_{3}$ of $\mathrm{dS}_{5}$ with line element

$$
\mathrm{d} \Omega_{3}^{2}=\mathrm{d} \omega_{1}^{2}+\sin ^{2} \omega_{1} \mathrm{~d} \omega_{2}^{2}+\sin ^{2} \omega_{1} \sin ^{2} \omega_{2} \mathrm{~d} \omega_{3}^{2},
$$

is not involved in the $H_{3}$ flux, the latter being invariant under its $\mathrm{SO}(4)$ rotations, and thus we set $\omega_{1}=\omega_{2}=\pi / 2$, while we wrap the string as $\omega_{3}=\nu \sigma$ to reinforce the stringy character of the embedding. Indeed, both wrappings - along $\xi$ and $\omega_{3}-$ turn out to play a crucial role in surfacing the full power of the $H_{3}$ dynamical contribution. Also, notice that having non-dynamical $\omega_{i}$ prevents the string soliton from boosting symmetrically as in $(2.3)$. Overall, the string embedding reads

$$
\begin{gathered}
r=r(\tau), \quad \chi=\chi(\tau), \quad \psi=\frac{\pi}{2}, \quad \xi=\kappa \sigma, \quad z=z(\tau), \\
\theta=\theta(\tau), \quad \phi=\phi(\tau), \quad \omega_{1}=\omega_{2}=\frac{\pi}{2}, \quad \omega_{3}=\nu \sigma,
\end{gathered}
$$

where $\kappa, \nu \in \mathbb{Z}$. Translating the $B_{2}$ field according to the map 2.7) and the above embedding as

$$
B_{2}=-\frac{\beta e^{-\phi} \sin \theta}{4}\left(r \sin ^{2} \chi \mathrm{d} r \wedge \mathrm{d} \xi+r^{2} \sin \chi \cos \chi \mathrm{d} \chi \wedge \mathrm{d} \xi\right)
$$

then the $\sigma$-model 3.32$)$ on the embedding 2.9$)$ reduces into the Lagrangian density 


$$
\begin{aligned}
\mathcal{L}=\frac{-\dot{r}^{2}-r^{2} \dot{\chi}^{2}+\kappa^{2} r^{2} \sin ^{2} \chi-\dot{z}^{2}}{z^{2}} & -\dot{\theta}^{2}+\sin ^{2} \theta \dot{\phi}^{2}+\nu^{2} \cos ^{2} \theta \\
& -\frac{\beta \kappa e^{-\phi} \sin \theta}{2 z}\left(r \sin ^{2} \chi \dot{r}+r^{2} \cos \chi \sin \chi \dot{\chi}\right)
\end{aligned}
$$

where the dot implies derivation wrt to the worldsheet time $\tau$. For our particular string embedding, the equations of motion for this Lagrangian are equivalent to those of the $\sigma$ model and read

$$
\begin{gathered}
4 \ddot{r}=\beta \kappa e^{-\phi} r \sin ^{2} \chi(z \sin \theta \dot{\phi}+\sin \theta \dot{z}-z \cos \theta \dot{\theta})-4 r\left(\kappa^{2} \sin ^{2} \chi-\dot{\chi}^{2}\right)+\frac{8 \dot{r} \dot{z}}{z}, \\
4 r \ddot{\chi}=\beta \kappa e^{-\phi} r \cos \chi \sin \chi(z \sin \theta \dot{\phi}+\sin \theta \dot{z}-z \cos \theta \dot{\theta})-4 r \kappa^{2} \cos \chi \sin \chi+\frac{8 r \dot{\chi} \dot{z}}{z}-8 \dot{r} \dot{\chi}, \\
4 z \ddot{z}=-\beta \kappa e^{-\phi} r z \sin \theta \sin \chi(\sin \chi \dot{r}+r \cos \chi \dot{\chi})+4 r^{2}\left(\kappa^{2} \sin ^{2} \chi-\dot{\chi}^{2}\right)+4\left(\dot{z}^{2}-\dot{r}^{2}\right), \\
4 \ddot{\theta}=2 \nu^{2} \sin 2 \theta+\beta \kappa e^{-\phi} r \cos \theta \sin \chi(\sin \chi \dot{r}+r \cos \chi \dot{\chi})-2 \sin 2 \theta \dot{\phi}^{2}, \\
4 \sin \theta \ddot{\phi}=-8 \cos \theta \dot{\theta} \dot{\phi}+\beta \kappa e^{-\phi} r \sin \chi(\sin \chi \dot{r}+r \cos \chi \dot{\chi}) .
\end{gathered}
$$

These equations are constrained by the worldsheet equation of motion, i.e. the Virasoro constraint

$$
\begin{aligned}
2 T_{\tau \tau} & =2 T_{\sigma \sigma}=\frac{\dot{r}^{2}+r^{2} \dot{\chi}^{2}+\kappa^{2} r^{2} \sin ^{2} \chi+\dot{z}^{2}}{z^{2}}-\sin ^{2} \theta \dot{\phi}^{2}+\dot{\theta}^{2}+\nu^{2} \cos ^{2} \theta=0, \\
T_{\tau \sigma} & =0
\end{aligned}
$$

The worldsheet energy-momentum tensor is conserved, $\nabla_{a} T^{a b}=0$, since $\partial_{\tau} T_{\tau \tau}=\partial_{\sigma} T_{\sigma \sigma}=0$ on the equations of motion 2.12 - 3.98). This compliance of the worldsheet constraints with the string coordinates' equations of motion yield, also, the consistency of our embedding.

Transforming into the Hamiltonian formulation, our worldsheet theory reduces to a simple particle system with conjugate momenta

$$
\begin{aligned}
& p_{r}=-\frac{2 \dot{r}}{z^{2}}-\frac{\beta \kappa e^{-\phi} \sin \theta}{2 z} r \sin ^{2} \chi, \quad p_{z}=-\frac{2 \dot{z}}{z^{2}}, \quad p_{\theta}=-2 \dot{\theta}, \\
& p_{\chi}=-\frac{2 r^{2} \dot{\chi}}{z^{2}}-\frac{\beta \kappa e^{-\phi} \sin \theta}{2 z} r^{2} \sin \chi \cos \chi, \quad p_{\phi}=2 \sin ^{2} \theta \dot{\phi}
\end{aligned}
$$


and Hamiltonian density

$$
\begin{aligned}
\mathcal{H}= & -\frac{z^{2}}{4 r^{2}}\left(p_{\chi}+\frac{\beta \kappa e^{-\phi} \sin \theta r^{2} \sin \chi \cos \chi}{2 z}\right)^{2}-\frac{z^{2}}{4}\left(p_{r}+\frac{\beta \kappa e^{-\phi} \sin \theta r \sin ^{2} \chi}{2 z}\right)^{2} \\
& -\frac{z^{2} p_{z}^{2}}{4}+\frac{p_{\phi}^{2}}{4 \sin ^{2} \theta}-\frac{p_{\theta}^{2}}{4}-\kappa^{2} r^{2} \sin ^{2} \chi-\nu^{2} \cos ^{2} \theta
\end{aligned}
$$

Of course, Hamilton's equations of motion on the above system coincide with the EulerLagrange equations 2.12 - 3.98). In this effective particle system, the masses are determined by the geometry and they can be read off through the kinetic terms. The string winding modes manifest themselves as a non-trivial potential on the particle dynamics, while the $\Omega$ deformation (i.e. the $H_{3}$ flux) is realized as a magnetic disturbance on the particle kinematics.

Before we proceed to analyze the dynamics, a crucial comment is in place. Usually, in this kind of Hamiltonian analysis on a string embedding we have a well-defined equation of motion for the target-space time, which always gives the energy of the string as its first integral and so on. Although not often emphasized, this is essential for a string state to be holographically associated with a dual operator, even if we don't know what that operator looks like. And we do desire a consistent holographic realization of our embedding, since we ultimately want to share the argument of (non-) integrability with the dual field theory as well. Hence, one should care about the validity of our embedding (and of every other embedding for that matter) on this kind of space. A first answer has already been provided through [94, where the string trajectories on the geometry (2.1) are shown to naturally realize the holographic principle. The second argument has to do with our particular formulation. The dual field theory lives on $\mathbb{R}^{4}$, in which the target-space time of our interest lives, i.e. $t \equiv x_{1}$. Since we have re-expressed $\mathbb{R}^{4}$ in the spherical coordinates 2.7 , then the radial coordinate $r$ should incorporate (Euclidean) time. Therefore, since we do include $r(\tau)$ into our dynamics, through the equation of motion (2.12), everything is in order and our string should have a well-defined holographic realization.

\subsection{A simple solution}

Next, we desire a simple solution on the equations of motion, around which we can study the fluctuations of the string. In that respect, regardless of having used the symmetries of the background to simplify our embedding (towards a less symmetric truncation), the equations of motion 2.12)-3.98 still possess a rich variety of simple solutions. However, not all of these solutions are consistent with our particular embedding: any consistent solution must also satisfy the worldsheet constraint (2.17). Given, in turn, the set of the consistent simple solutions, not all of those are actually useful since not all of them permit fluctuations 
that include the $B_{2}$ field contribution on the dynamics. The latter being the only possible non-integrable deviation from the integrable $\mathrm{H}_{5} \times \mathrm{dS}_{5}$. The associated $H_{3}$ flux dynamics is reflected on the $\beta$-dependent terms in (2.12)- 3.98$)$, thus our simple solution should let those terms survive in our fluctuating equations.

Under the above considerations, it turns out that there is an infinite set of invariant planes that do the job, for $\theta \in\left(\frac{\pi}{4}, \frac{\pi}{2}\right) \cup\left(\frac{\pi}{2}, \frac{3 \pi}{4}\right)$ and $\chi \in(0, \pi)$. It may seem naively odd, but the most - by far - convenient choice comes with the invariant plane

$$
\left\{r=\dot{r}=\ddot{r}=0, \chi=\frac{\pi}{2}, \dot{\chi}=\ddot{\chi}=0, \theta=\theta_{\star} \equiv \arctan \sqrt{\frac{5}{3}}, \dot{\theta}=\ddot{\theta}=0\right\},
$$

around which the fluctuations simplify tremendously. On this plane, the equations of motion (2.12)-3.98) are satisfied along with the simple solutions

$$
\phi(\tau)=-\nu \tau, \quad z(\tau)=\frac{e^{\frac{\nu}{2} \tau}}{\sqrt{10}}
$$

where the coefficients including the winding number $\nu$ were identified by the Virasoro constraint 2.17), while the signs and the constants were selected to our convenience without loss of generality

Note that the symmetry analysis on the background was not necessary to build an embedding. We just used it to shape a less symmetric string truncation, so as to have a better chance in non-integrability. Had we not used those symmetry considerations, we would have chosen a far more general embedding whose equations of motion would include a large variety of invariant planes. Nevertheless, all of those planes would eventually descend down to the invariant plane 2.20) and its corresponding simple solution (2.21) as the only useful option, just through a way more laborious path.

Next, we expand around the invariant plane in order to study the dynamical behavior of the system there. While the $r, \chi$ and $\theta$ fluctuations around the plane are generally coupled, such complexity is not eventually needed in our case. Stated otherwise, we shall study isolated fluctuations on each one of those dimensions, around the invariant plane 2.20 and on the simple solution (2.21). As in the last chapter, we call such a fluctuation a Normal Variational Equation (NVE).

\footnotetext{
${ }^{4}$ To be precise, there is another choice of signs that gives a similar result, while the rest of the choices turn out quite complicated.
} 


\subsection{Fluctuations around the invariant plane}

To isolate the $\theta$-fluctuations around the invariant plane 2.20$)$, we expand as $\theta(\tau)=\theta_{\star}+\epsilon \vartheta(\tau)$ for $\epsilon \rightarrow 0$ in the $\theta$-equation of motion (2.15), while we keep the other dimensions frozen, i.e. $\left\{r=\dot{r}=\ddot{r}=0, \chi=\frac{\pi}{2}, \dot{\chi}=\ddot{\chi}=0\right\}$. Hence, we obtain the $\theta$-NVE

$$
\ddot{\vartheta}(\tau)=0
$$

which has a Liouvillian solution.

In the same vein, the isolated $\chi$-fluctuations around the invariant plane occur for $\chi(\tau)=$ $\frac{\pi}{2}+\epsilon x(\tau)$ while $\left\{r=\dot{r}=\ddot{r}=0, \theta=\theta_{\star}, \dot{\theta}=\ddot{\theta}=0\right\}$, which however solves the $\chi$-equation of motion 2.13 identically and gives no further insight.

Therefore, we are only left with the $r$-fluctuations around the invariant plane 2.20). To isolate those, we expand as $r(\tau)=0+\epsilon \varrho(\tau)$ for $\epsilon \rightarrow 0$ in the $r$-equation of motion 2.12, while we keep the other dimensions frozen, i.e. $\left\{\chi=\frac{\pi}{2}, \dot{\chi}=\ddot{\chi}=0, \theta=\theta_{\star}, \dot{\theta}=\ddot{\theta}=0\right\}$. Hence, we obtain the $r$-NVE

$$
\ddot{\varrho}(\tau)-\nu \dot{\varrho}(\tau)+\kappa\left(\kappa+\frac{\beta \nu e^{\frac{3 \nu}{2} \tau}}{32}\right) \varrho(\tau)=0,
$$

which is solved for

$$
\begin{aligned}
& \varrho(\tau)=c_{1} \mathrm{~J}_{G}(f(\tau)) e^{\frac{\nu \tau}{2}} \Gamma(1+G)+c_{2} \mathrm{~J}_{-G}(f(\tau)) e^{\frac{\nu \tau}{2}} \Gamma(1-G), \\
& f(\tau)=\sqrt{\frac{\beta \kappa e^{\frac{3 \nu \tau}{2}}}{18 \nu}}, \quad G=\frac{2 \sqrt{-4 \kappa^{2}+\nu^{2}}}{3 \nu}
\end{aligned}
$$

where $c_{1}, c_{2}$ are constants and $\mathrm{J}_{n}(\tau), \Gamma(z)$ are the Bessel function of the first kind and the gamma function, respectively. Before anything, two comments are in place here. First, if the string windings are such that $\kappa \nu<0$, then $f(\tau) \in \mathbb{I}$ and we just work with the modified Bessel functions. Secondly, if the windings are such that $G \in \mathbb{I}$ then $\mathrm{J}_{n}(\tau)$ acquires a purely imaginary order $n \in \mathbb{I}$ and gives a complex number $z_{1}(\tau) \in \mathbb{C}$, while its conjugate function $\mathrm{J}_{-n}(\tau)$ gives $z_{1}^{\star}(\tau)$. Similarly, $\Gamma(z)$ with $z \in \mathbb{C}$ gives a complex number $z_{2} \in \mathbb{C}$, while $\Gamma\left(z^{\star}\right)$ gives $z_{2}^{\star}$. Thus, for $G \in \mathbb{I}$, our $\varrho$-solution 2.24 can be written as

$$
\varrho(\tau)=c_{1} e^{\frac{\nu \tau}{2}} z_{1}(\tau) z_{2}+c_{2} e^{\frac{\nu \tau}{2}} z_{1}^{\star}(\tau) z_{2}^{\star},
$$


which can only be real for $c_{1}=c_{2}$. This is a necessary condition for the physicality of our solution.

The Bessel function is non-Liouvillian except only for half integer order $n$. If $n= \pm G$ is imaginary then it can never be a half integer, anyway. If it is real, on the other hand, $\pm G$ reflects the various configurations of our embedding and thus it cannot be restricted without losing generality. In other words, we should care about the solution (2.24) on every value of the winding numbers $\kappa, \nu$. Even if there are particular string configurations (for appropriate $\kappa, \nu)$ that are Liouvillian, there are always others that are not. Hence, we have ultimately spotted a string embedding that exhibits non-integrable dynamics.

As a consistency check, note that for $\beta=0$ in 2.23 we recover integrability, as we should for an undeformed and symmetric vacuum. The same holds for $\kappa, \nu=0$, where the string reduces to a point particle on $\mathrm{H}_{5} \times \mathrm{dS}_{5}$ that cannot feel the $H_{3}$ flux.

\subsection{A simpler embedding}

Since one is never enough, we shall study another string embedding. We have already mentioned that had we included extra string coordinate dependence than the one we chose before, we would have ultimately ended up studying the embedding (2.9). Hence, we are led to build a simpler truncation this time. It turns out that the most minimal alternative is to localize the coordinates $z=z_{0}=1$ and $\chi=\frac{\pi}{2}$ in our previous embedding, which now becomes

$$
\begin{aligned}
& r=r(\tau), \quad \chi=\frac{\pi}{2}, \quad \psi=\frac{\pi}{2}, \quad \xi=\kappa \sigma, \quad z=1, \\
& \theta=\theta(\tau), \quad \phi=\phi(\tau), \quad \omega_{1}=\omega_{2}=\frac{\pi}{2}, \quad \omega_{3}=\nu \sigma,
\end{aligned}
$$

where $\kappa, \nu \in \mathbb{Z}$. The $B_{2}$ field that couples to the new embedding reduces to

$$
B_{2}=-\frac{\beta}{4} r e^{-\phi} \sin \theta \mathrm{d} r \wedge \mathrm{d} \xi
$$

while the associated Lagrangian density becomes

$$
\mathcal{L}=-\dot{r}^{2}+\kappa^{2} r^{2}-\dot{\theta}^{2}+\sin ^{2} \theta \dot{\phi}^{2}+\nu^{2} \cos ^{2} \theta-\frac{\beta \kappa}{2} r e^{-\phi} \sin \theta \dot{r}
$$

Of course, on this embedding too, the equations of motion for this Lagrangian are equivalent to those of the $\sigma$-model and read 


$$
\begin{gathered}
4 \ddot{r}=-4 \kappa^{2} r+\beta \kappa r e^{-\phi}(\sin \theta \dot{\phi}-\cos \theta \dot{\theta}), \\
4 \ddot{\theta}=2 \sin 2 \theta\left(\nu^{2}-\dot{\phi}^{2}\right)+\beta \kappa e^{-\phi} \cos \theta r \dot{r}, \\
4 \sin \theta \ddot{\phi}=-8 \cos \theta \dot{\theta} \dot{\phi}+\beta \kappa e^{-\phi} r \dot{r} .
\end{gathered}
$$

These equations are constrained by the worldsheet equation of motion, i.e. the Virasoro constraint

$$
\begin{aligned}
2 T_{\tau \tau} & =2 T_{\sigma \sigma}=\dot{r}^{2}+\kappa^{2} r^{2}-\sin ^{2} \theta \dot{\phi}^{2}+\dot{\theta}^{2}+\nu^{2} \cos ^{2} \theta=0 \\
T_{\tau \sigma} & =0 .
\end{aligned}
$$

The worldsheet energy-momentum tensor is conserved, $\nabla_{a} T^{a b}=0$, since $\partial_{\tau} T_{\tau \tau}=\partial_{\sigma} T_{\sigma \sigma}=0$ on the equations of motion 2.29)-2.31, yielding also the consistency of our embedding. Of course, the associated Hamiltonian system here is qualitatively the same as with the previous embedding.

In this particular case however, under the considerations - again - of consistency and of including the $H_{3}$ flux contribution, there is only one invariant plane that serves our cause. That is

$$
\left\{r=\dot{r}=\ddot{r}=0, \theta=\frac{\pi}{4}, \dot{\theta}=\ddot{\theta}=0\right\}
$$

Note that, while for the previous embedding the choice $\theta=\frac{\pi}{4}$ was excluded since it led to useless invariant planes, here it constitutes our only option. This is indeed the unique plane that does the job and on which the equations of motion (2.29)-2.31) are satisfied, along with the simple solution

$$
\phi(\tau)=-\nu \tau,
$$

where the coefficient was identified with the winding number $\nu$ through the Virasoro constraint 2.32), while the sign was again selected to our convenience without loss of generality. For one last time, we move on to study the isolated fluctuations around the invariant plane (2.33) and on its associated simple solution 2.34).

Obviously, the $\theta$-fluctuations are again trivial and so we are left to study the fluctuations along $r$. We expand $r(\tau)=0+\epsilon \varrho(\tau)$ for $\epsilon \rightarrow 0$ in the $r$-equation of motion (2.29), while we 
keep $\theta$ frozen, i.e. $\left\{\theta=\frac{\pi}{4}, \dot{\theta}=\ddot{\theta}=0\right\}$. Hence, we obtain the $r$-NVE

$$
\dddot{\varrho}(\tau)+\kappa\left(\kappa+\frac{\sqrt{2} \beta \nu e^{\nu \tau}}{8}\right) \varrho(\tau)=0
$$

which is solved for

$$
\begin{aligned}
& \varrho(\tau)=c_{1} \mathrm{~J}_{G}(f(\tau)) \Gamma(1+G)+c_{2} \mathrm{~J}_{-G}(f(\tau)) \Gamma(1-G), \\
& f(\tau)=\sqrt{\frac{\beta \kappa e^{\nu \tau}}{\sqrt{2} \nu}}, \quad G=\frac{2 i \kappa}{\nu},
\end{aligned}
$$

where $c_{1}, c_{2}$ are constants and $\mathrm{J}_{n}(\tau), \Gamma(z)$ are the Bessel function of the first kind and the gamma function, respectively. Again, if the string windings are such that $\kappa \nu<0$, then $f(\tau) \in \mathbb{I}$ and we just work with the modified Bessel functions. Also, as explained for the case of the previous solution (2.24), since the order $n= \pm G$ of the Bessel function is purely imaginary, it can never be a half integer (that gives a Liouvillian solution) while it must necessarily hold that $c_{1}=c_{2}$ for the physicality of our solution (2.36). Hence, we have spotted another non-integrable fluctuation of the string.

Again, as a consistency check, note that for $\beta=0$ in 2.35 we recover integrability, as we should for the undeformed vacuum. The same holds for $\kappa, \nu=0$, where the string reduces to a point particle on $\mathrm{H}_{5} \times \mathrm{dS}_{5}$ that does not couple to the Kalb-Ramond field.

As indicated repeatedly, the invariant planes we have studied so far are the unique solutions that consistently incorporate the $H_{3}$ flux contribution. Nevertheless, in case we want to be persistent and make the non-integrable character of the system manifest in an additional way, we could go for a more involved string embedding. In particular, we could build a spinning string by letting

$$
\xi(\tau, \sigma)=\kappa \sigma+\Xi(\tau), \quad \omega_{3}(\tau, \sigma)=\nu \sigma+\Omega(\tau),
$$

in the previous embeddings, 2.9 and 2.26). Choosing that truncation, worldsheet consistency conditions (on necessarily similar invariant planes) drop the dynamics down to the exact same results we found for the simpler embeddings.

As an additional consistency check, we can repeat everything we have done so far in Euclidean signature, i.e. on the Euclidean $\operatorname{AdS}_{5} \times \mathrm{S}^{5}$. In order to do this, we Wick rotate the target space in (2.1) as $\phi \rightarrow i \varphi$ while we pick - for consistency - an also Euclidean worldsheet. Again, we acquire the exact same results up to certain factors. 
Since an integrable structure exhibits its homonymous property on all of its sectors, we deduce that the dynamical sector we studied and, therefore, the whole supergravity background under consideration are classically non-integrable.

\section{Conclusions}

Ultimately, we have proven that string theory on the vacuum dual to the $\Omega$-deformed $\mathcal{N}=4$ SYM, recently proposed in [35], is classically non-integrable in the Liouvillian sense. Using the broken symmetries (by the $H_{3}$ flux) of the background, we constructed appropriate string embeddings and studied their fluctuations around simple solutions of their equations of motion. Since particular fluctuations turned out to be non-Liouvillian for a general string configuration, we declared the whole theory as non-integrable.

Notice that, contrary to the usual method of analytic non-integrability, on this particular analysis we did not have to enforce differential Galois theory and Kovacic's theorem on differential equations of motion. That is, we reached exact non-Liouvillian solutions given in terms of the Bessel function of the first kind, of no half-integer order for a general string configuration.

Since the supergravity background we examined is dual to the $\Omega$-deformed $\mathcal{N}=4 \mathrm{SYM}$, holography dictates that the statement of non-integrability must be shared by the gauge theory as well. Hence, apart from being just a delicate Hamiltonian mechanics problem, the present work suggests that the $\Omega$-deformation does not preserve classical integrability.

However, a non-integrable theory may possess integrable subsectors or limits. In the $\Omega$-deformed theory, this is obviously true on the grounds of the existing literature that associates this deformation with various integrable structures, as noted in the introduction. Therefore, the ontology of the regimes of integrability is worthy of further examination. More interestingly though, given the $\Omega$ dual background (2.1), a valuable study would be based on its Kalb-Ramond field which realizes the $\Omega$-deformation itself. In particular, special vacua or limits of string theory on this supergravity background could investigate the action of this $B_{2}$ field on the associated string states, while - in that case - holography should be in place to shed light on their dual $\Omega$-deformed field theory subsectors.

\section{Symmetries of the $\Omega$ vacuum}

We are interested in the $\Omega$ vacuum $[35], H^{5} \times \mathrm{dS}_{5}$, namely

$$
\mathrm{d} s^{2}=\frac{\mathrm{d} \vec{x}_{4}^{2}+\mathrm{d} z^{2}}{z^{2}}-\sin ^{2} \theta \mathrm{d} \phi^{2}+\mathrm{d} \theta^{2}+\cos ^{2} \theta \mathrm{d} \Omega_{3}^{2},
$$


where we have set both the radii equal to one. This geometry enjoys an $\mathrm{SO}(1,5) \times \mathrm{SO}(1,5)$ isometry and comes along with an NS-NS field

$$
B_{2}=-\frac{\beta}{4} \frac{e^{-\phi} \sin \theta}{z}\left(\mathrm{~d} x_{1} \wedge \mathrm{d} x_{2}+\mathrm{d} x_{3} \wedge \mathrm{d} x_{4}\right)
$$

where $\beta \in \mathbb{R}$ parametrizes (linearly) the $\Omega$-deformation of the dual field theory. For later convenience in calculating the symmetries separately in the two subspaces, $H^{5}$ and $\mathrm{dS}_{5}$, we rewrite $B_{2}$ as

$$
B_{2}=f(\phi, \theta) \frac{\omega_{2}}{z}, \quad f(\phi, \theta)=-\frac{\beta}{4} e^{-\phi} \sin \theta,
$$

where $\omega_{2}$ is an invariant 2-form in $\mathbb{R}^{4}$ of $H^{5}$. Obviously, the $H_{3}=\mathrm{d} B_{2}$ flux does not respect all the symmetries of the gravitational field, which prompt us to investigate the actual symmetry vector flow that is shared by both fields.

Two observations are in place here. First, we note that the geometry (2.38) is a product space and, thus, its Killing vectors (KVs) are decoupled for the two subspaces. This lets us vary $H_{3}$ along $H^{5}$ or $\mathrm{dS}_{5}$, separately. The second point is that we can approach the problem through two distinct paths: we can check which of the KVs are respected by $H_{3}$ or we can independently find the symmetries of $H_{3}$, regardless if they are KVs or not. However, the independent symmetries of $\mathrm{H}_{3}$ will involve vectors that mix up the whole set of coordinates and the calculation (of the Lie derivative) will become quite involved. Therefore, we stick to the first option.

Let $K_{1}$ be the symmetries of $H^{5}$ along $H_{3}$, then it holds that

$$
\begin{aligned}
0=\mathcal{L}_{K_{1}} H_{3} & =d i_{K} H_{3}+\underline{i}_{K_{1}} d H_{3} \\
& =d i_{K_{1}}\left(\mathrm{~d} f \wedge \frac{\omega_{2}}{z}+f d\left(\frac{\omega_{2}}{z}\right)\right) \\
& =d f \wedge d i_{K_{1}}\left(\frac{\omega_{2}}{z}\right)+d f \wedge i_{K_{1}} d\left(\frac{\omega_{2}}{z}\right)+f d i_{K_{1}} d\left(\frac{\omega_{2}}{z}\right)
\end{aligned}
$$

where the first two terms must vanish separately from the last, leading to the conditions

$$
\mathcal{L}_{K_{1}}\left(\frac{\omega_{2}}{z}\right)=0, \quad \mathcal{L}_{K_{1}}\left(\frac{\mathrm{d} z \wedge \omega_{2}}{z^{2}}\right)=0
$$

Now, let $K_{2}$ be the symmetries along $\mathrm{dS}_{5}$, then it holds that 


$$
\begin{aligned}
0=\mathcal{L}_{K_{2}} H_{3} & =d i_{K_{2}} H_{3}+\underline{i}_{K_{2}} d H_{3} 0 \\
& =d i_{K_{2}}\left(\mathrm{~d} f \wedge \frac{\omega_{2}}{z}+f d\left(\frac{\omega_{2}}{z}\right)\right) \\
& =d\left[\left(i_{K_{2}} d f\right) \frac{\omega_{2}}{z}\right]
\end{aligned}
$$

which leads to the condition

$$
\mathcal{L}_{K_{2}} f=i_{K_{2}} d f=0 \text {. }
$$

Therefore, in order to study the above conditions we have to find the KVs of each subspace.

\section{D.1 Constant KVs of $H^{5}$ along the $H_{3}$}

$H^{5}$ enjoys an $\mathrm{SO}(1,5)$ isometry, where the $\mathrm{SO}(5)$ subgroup implies ten rotations supplemented by five $\mathrm{SO}(1,1)$ conformal transformations. The latter, as we are about to see, imply one dilation and four special conformal transformations.

For a symmetric space the KVs are easy to find, since we can always realize it as an embedding in a higher dimensional flat space, which inherits the rotational group on its hypersurface. In our case, $H^{5}$ is a hyperboloid in $\mathbb{R}^{6}$ that inherits its $\mathrm{SO}(1,5)$ isometry from the original $\mathrm{SO}(6)$.

Therefore, we consider the rotations of $\mathbb{R}^{6}$

$$
V_{i} \equiv V_{i}^{A} \partial_{Y^{A}}
$$

where $Y^{A}, A=0, \ldots, 5$ are the embedding coordinates which build the hypersurface

$$
\eta_{A B} Y^{A} Y^{B}=-1
$$

with $\eta_{A B}=\operatorname{diag}(1,1, \ldots,-1)$. These rotations are inherited into $H^{5}$ as the KVs

$$
K_{i} \equiv K_{i}^{\mu} \partial_{\mu}=g^{\mu \nu}\left(\frac{\partial Y^{A}}{\partial x^{\nu}} V_{A}\right)_{i} \partial_{\mu}
$$

where $x^{\mu}$ are the $H^{5}$ coordinates and $g_{\mu \nu}$ its metric, while $i$ runs in the KV space. By choosing the Poicarè solution to eq.2.44, 


$$
\begin{aligned}
Y^{0} & =\frac{z}{2}\left(1+\frac{1}{z^{2}}\left(1+\sum_{i=1}^{4} x_{i}^{2}\right)\right), \\
Y^{i} & =\frac{x_{i}}{z}, \quad i=1, \ldots, 4, \\
Y^{5} & =\frac{z}{2}\left(1-\frac{1}{z^{2}}\left(1-\sum_{i=1}^{4} x_{i}^{2}\right)\right),
\end{aligned}
$$

one can find each one of the fifteen $\mathrm{KVs}$ of $H^{5}$. The first in line are the expected six rotations of the $\mathrm{SO}(4)$ subgroup, namely

$$
K_{R_{i j}}=x_{i} \partial_{j}-x_{j} \partial_{i}
$$

for $i, j=1, \ldots, 4$, defined up to an overall sign.

Next, there are eight KVs composed of the two sets of conformal Killing vectors (CKVs)

$$
\begin{aligned}
\frac{K_{C_{k}}=K_{C_{k}}{ }^{\mu} \partial_{\mu}:}{K_{C_{k}}{ }^{k}} & =\frac{1-x_{k}^{2}+\sum_{i \neq k} x_{i}^{2}+z^{2}}{2}, \\
K_{C_{k}}{ }^{i} & =-x_{k} x_{i}, \\
K_{C_{k}}{ }^{z} & =-x_{k} z, \\
\frac{K_{V_{k}}=K_{V_{k}}{ }^{\mu} \partial_{\mu}:}{K_{V_{k}}{ }^{k}} & =\frac{1+x_{k}^{2}-\sum_{i \neq k} x_{i}^{2}-z^{2}}{2}, \\
K_{V_{k}}{ }^{i} & =x_{k} x_{i}, \\
K_{V_{k}}{ }^{z} & =x_{k} z,
\end{aligned}
$$

one for each value of $k=1, \ldots, 4$, again up to overall signs, accompanied by the unique element of the homothetic group for $H^{5}$, namely the $\mathrm{SO}(1,1)$ dilation

$$
K_{D}=\sum_{i=1}^{4} x_{i} \partial_{i}+z \partial_{z}
$$

While the six rotations and the dilation are in their standard form, the eight CKVs look certainly uglier. This can be easily fixed by a change of their basis. This is achieved by adding 
and subtracting every vector of the first set, $K_{C_{k}}$, with its $k$-counterpart in the second set, $K_{V_{k}}$. By adding them, we acquire the four (conformal) translations 5

$$
K_{S C_{i}}=\partial_{i}, \quad i=1, \ldots, 4,
$$

up to a sign, as expected by the $x_{i}$-invariance of the metric tensor. Apart from the dilation, these are the remaining four $\mathrm{SO}(1,1) \mathrm{SCKVs}$. The last four KVs are found by subtraction and they are what remains of the $\mathrm{SO}(5)$ (conformal) rotations, namely

$$
K_{C R_{k}}=\left(x_{k}^{2}-\sum_{i \neq k} x_{i}^{2}-z^{2}\right) \partial_{k}-\sum_{i \neq k} 2 x_{k} x_{i} \partial_{i}-2 x_{k} z \partial_{z}
$$

for $k=1, \ldots, 4$ and, as always, up to an overall sign.

Without further ado, we plug all of the above KVs into the conditions for invariance along the $H_{3}$ flux, eq.2.41, and observe that the only ones that satisfy them are

$$
\begin{aligned}
& K_{R_{12}}=x_{1} \partial_{2}-x_{2} \partial_{1} \\
& K_{R_{34}}=x_{3} \partial_{4}-x_{4} \partial_{3} \\
& K_{S C_{i}}=\partial_{i} \quad i=1, \ldots, 4
\end{aligned}
$$

namely two rotations of the $\mathrm{SO}(4)$ subgroup rotating the $\mathbb{R}^{4}$ and the four $\mathrm{SO}(1,1) \mathrm{SCKV}$.

\section{D.2 Constant KVs of $\mathrm{dS}_{5}$ along the $H_{3}$}

This case is simpler, since we don't have to find the $\mathrm{KVs}_{\mathrm{s}}$ of $\mathrm{dS}_{5}$. The condition for invariant $\mathrm{dS}_{5}$ vectors along $H_{3}$, eq. 2.42 , has quite a simple form and solution. Thus, it is easier to just use this condition to find the $H_{3}$ symmetries and then constrain them to be KVs of $\mathrm{dS}_{5}$.

Taking the condition

$$
\mathcal{L}_{K} f=i_{K} d f=0
$$

and observing that

$$
d f=e^{-\phi}(\cos \theta \mathrm{d} \theta-\sin \theta \mathrm{d} \phi),
$$

we can easily conclude that the minimal ansatz for a symmetry vector is

\footnotetext{
${ }^{5} K=\partial_{i}, i=1, \ldots, 4$, are translations in $\mathbb{R}^{4}$ and special conformal transformations in $H^{5}$.
} 


$$
K=A(\phi, \theta) \partial_{\phi}+B(\phi, \theta) \partial_{\theta},
$$

which solves eq. 2.53) for

$$
K=A(\phi, \theta) \partial_{\phi}+A(\phi, \theta) \tan \theta \partial_{\theta},
$$

the simpler choice for which is for $A(\phi, \theta)=1$, namely

$$
K=\partial_{\phi}+\tan \theta \partial_{\theta}
$$

However, this family of vectors, eq.2.56), fails to satisfy the Killing equation $K_{(\mu ; \nu)}=0$. The resolution comes by observing the way the Killing equation fails and is actually quite straightforward. We consider the next most-minimal symmetry vector

$$
K=A\left(\phi, \theta, \omega_{1}\right) \partial_{\phi}+B\left(\phi, \theta, \omega_{1}\right) \partial_{\theta}+C\left(\phi, \theta, \omega_{1}\right) \partial_{\omega_{1}},
$$

where $\omega_{1}$ is an angle in the 3 -sphere, $\mathrm{d} \Omega_{3}^{2}=\mathrm{d} \omega_{1}^{2}+\sin ^{2} \omega_{1} \mathrm{~d} \Omega_{2}^{2}$, of $\mathrm{dS}_{5}$. Plugging this vector into the condition eq.2.53), it gets restricted to

$$
K=A\left(\phi, \theta, \omega_{1}\right) \partial_{\phi}+A\left(\phi, \theta, \omega_{1}\right) \tan \theta \partial_{\theta}+C\left(\phi, \theta, \omega_{1}\right) \partial_{\omega_{1}}
$$

Next, we surrender it to the Killing equation for reformation. The $K_{(\phi ; \phi)}=0$ component gives that

$$
\frac{\partial A}{\partial \phi}=-A \quad \Rightarrow \quad A\left(\phi, \theta, \omega_{1}\right)=e^{-\phi} A_{1}(\theta, \omega)
$$

while the $K_{(\theta ; \theta)}=0$ component gives that

$$
A_{1}=-\frac{\partial A_{1}}{\partial \theta} \sin \theta \cos \theta \quad \Rightarrow \quad A_{1}\left(\theta, \omega_{1}\right)=\cot \theta A_{2}\left(\omega_{1}\right) .
$$

Finally, the $K_{\left(\omega_{1} ; \omega_{1}\right)}=0$ component gives

$$
\frac{\partial C}{\partial \omega_{1}}=e^{-\phi} \tan \theta A_{2}\left(\omega_{1}\right) \Rightarrow\left\{\begin{array}{c}
\frac{\partial C_{1}\left(\omega_{1}\right)}{\partial \omega_{1}}=A_{2}\left(\omega_{1}\right) \\
C_{2}(\phi, \theta)=\tan \theta
\end{array}\right.
$$


which, along with the $K_{\left(\phi, \omega_{1}\right)}=K_{\left(\theta, \omega_{1}\right)}=0$ components that demand

$$
\frac{\partial A_{2}\left(\omega_{1}\right)}{\partial \omega_{1}}=-C_{1}\left(\omega_{1}\right)
$$

specifies the last subfunctions as $A_{2}\left(\omega_{1}\right)=\cos \omega_{1}$ and $C_{1}\left(\omega_{1}\right)=\sin \omega_{1}$. The rest of the components of the Killing equation are also satisfied and, therefore, our KV becomes

$$
K_{B}=e^{-\phi} \cot \theta \cos \omega_{1} \partial_{\phi}+e^{-\phi} \cos \omega_{1} \partial_{\theta}+e^{-\phi} \tan \theta \sin \omega_{1} \partial_{\omega_{1}}
$$

and is identified as one of the $\mathrm{SO}(1,1)$ boosts of the $\mathrm{SO}(1,5)$ isometry of $\mathrm{dS}_{5}$. Had we chosen a different angle than $\omega_{1}$ from the 3 -sphere of $\mathrm{dS}_{5}$, i.e. $\omega_{2}$ or $\omega_{3}$, we would have found two more boost KVs. These are the unique non-trivial KVs of $\mathrm{dS}_{5}$ that preserve the $H_{3}$ flux.

The rest of the KVs of $\mathrm{dS}_{5}$ that preserve $H_{3}$ are trivial, since the flux is entirely $\Omega_{3^{-}}$ invariant, and consist of the six $\mathrm{SO}(4)$ rotations of $\Omega_{3}$ inside $\mathrm{dS}_{5}$. This concludes the symmetry analysis on $H_{3}$. 



\section{Chapter 3}

\section{Integrability on $\mathrm{AdS}_{7}$ vacua}

\section{Introduction}

Quantum field theories may be realized in the context of renormalization group (RG) flows, from short distances in the UV to long distances in the IR. The endpoints of these flows are called fixed points and the associated field theories are scale invariant. In $d$-dimensional relativistic field theories with $\mathrm{SO}(1, d-1)$ spacetime symmetry, it is common to assume that the fixed-point theory is a CFT, whose symmetry is enhanced to the conformal algebra $\mathrm{SO}(2, d)$. Aside from free CFTs, there is compelling evidence for a vast landscape of interacting CFTs in diverse dimensions, with many of these theories being non-Lagrangian. The latter are CFTs without a known representation in terms of fields and, in this sense, the best way to learn about those field theories is to employ the AdS/CFT duality [11] and work on their holographically dual vacua. SCFTs in six dimensions, which are the subject of this chapter, resist a Lagrangian realization.

Examples of such six-dimensional theories include $\mathcal{N}=(2,0)$ theories $[9]$, realized on a stack of M5-branes. Less is known about the $\mathcal{N}=(1,0)$ theories. None of those sixdimensional CFTs has a weakly-coupled UV Lagrangian. However, one may always move along the tensor branch of the theory, which corresponds to giving a VEV to the scalar inside the tensor multiplet. In such cases, one can find an effective Lagrangian description for $\mathcal{N}=(1,0)$ theories in terms of a weakly-coupled quiver gauge theory, where the scalars inside the tensor multiplets - controlling the coupling constants of the corresponding gauge groupsare promoted to a set of dynamical fields. Reaching for the origin of the tensor branch typically leads to a strongly-coupled six-dimensional SCFT with $\mathcal{N}=(1,0)$ supersymmetry. Some of those systems have a realization in string theory [95], 96]. The description of these CFTs is well-advanced, see for example [36 43 and 97 107], which deal with the system from the field-theoretical, D-brane or holographic point of view.

The subject of this chapter is to study classical integrability on such six-dimensional $\mathcal{N}=(0,1)$ SCFTs through their holographically-dual supergravity vacua, which were developed in 36 43. Those massive type IIA backgrounds preserve $\mathcal{N}=(0,1)$ supersymmetry, 
they consist of a warped $\mathrm{AdS}_{7} \times \mathcal{M}^{3}$ geometry, where $\mathcal{M}^{3}$ is isomorphic to $\mathrm{S}^{3}$, and are associated with D8-D6-NS5 Hanany-Witten brane set-ups. While the study of a particular bosonic string soliton illustrates that all vacua with a warped geometry exhibit complete non-integrability, in the special case of the unwarped $\mathrm{AdS}_{7} \times \mathcal{M}^{3}$ space we prove the opposite to be true. That is, we observe that the Wess-Zumino-Witten (WZW) model on $\mathcal{M}^{3}$ is an integrable deformation of the same model on $\mathrm{S}^{3}$, which yields that the Neveu-Schwarz sector of the string $\sigma$-model on this special vacuum is classically integrable.

This chapter has the following structure. In Section 2, we summarize the string and field-theoretical realizations and describe the holographic map between those pictures that follow from a Hanany-Witten description 68 of the CFTs. In Section 3, we investigate classical integrability by studying the dynamical evolution of a semiclassical, bosonic string soliton. As in the previous chapters, we employ out tools of analytic non-integrability and prove that all vacua with a warped $\mathrm{AdS}_{7} \times \mathcal{M}^{3}$ geometry are non-integrable. However, the special case of an unwarped space is singled out, on which the string equations of motion exhibit a Liouvillian solution, yielding the possibility of an integrable vacuum. Indeed, in Section 4 we show that the WZW model on $\mathcal{M}^{3}$ is a $\lambda$-deformation of the same model on $\mathrm{S}^{3}$, which, together with the fact that $\mathrm{AdS}_{7}$ is a symmetric space, means that the string $\sigma$-model is classically integrable on $\mathrm{AdS}_{7} \times \mathcal{M}^{3}$. Of course, this is supported by the construction of an explicit Lax connection, through which the equations of motion of the string on the special vacuum are derived. In Section 4.3, we give some of the field theoretical observables associated with this special, integrable background, which serve as a holographic definition of the dual integrable $\mathcal{N}=(1,0)$ six-dimensional SCFT and, finally, we summarize our findings and conclusions in Section 5 ,

\section{Six-dimensional SCFT and holography}

It is useful to recap the main issues afflicting higher dimensional $(d>4)$ field theories. Consider a simple interacting field theory in six dimensions with action,

$$
S=\int d^{6} x\left[-\frac{1}{2}\left(\partial_{\mu} \phi\right)^{2}-V(\phi)\right] .
$$

Here $\phi$ represents a real scalar field with classical dimension $[\phi]=m^{2}$. The potential can be a mass term $V=\frac{m^{2}}{2} \phi^{2}$ or more interestingly a classically marginal interaction term, like $V=g \phi^{3}$, but this would lead to a system without ground state (for $\phi<0$ ). On the other hand, a potential like $V=\lambda \phi^{4}$ has a well-defined vacuum, but the interaction is irrelevant, hence the theory is not well-defined in small scales, i.e. it has no UV completion. The Wilsonian logic, according to which we start from a conformal (not necessarily weakly 
coupled) field theory and deform it by inserting relevant operators into the Lagrangian, flowing to interesting field theories at low energies, does not seem to apply here.

Nevertheless, different string theoretic constructions have suggested that supersymmetric field theories of scalars coupled to gauge fields have an interacting UV fixed point. In fact, considering a Lagrangian like

$$
\mathcal{L} \sim-\frac{1}{2}\left(\partial_{\mu} \phi\right)^{2}-c \phi F_{\mu \nu}^{2}+\text { fermions }
$$

and assuming $\langle\phi\rangle \rightarrow 0$, we are dealing with the strong coupling limit of a gauge field theory (since the scalar $\phi$ takes the role of the inverse coupling of the gauge theory). The presence of fermions in the supersymmetric theory implies the possible existence of gauge anomalies that need to be canceled. This cancellation is possible if the scalar $\phi$ belongs to a tensor multiplet 107], [108 and a certain tuning between the amount of adjoint and fundamental matter must be imposed.

This picture was realised in D-brane constructions. The relevant Hanany-Witten setups 68 were presented in 109. The associated six-dimensional field theories preserve eight Poincare supercharges and have $\mathrm{SO}(1,5)$ Lorentz and $\mathrm{SU}(2)$ R-symmetries. In more detail, the field theories with $\mathcal{N}=(1,0)$ supersymmetry are constructed in terms of the following multiplets:

- Tensor multiplets with field content $\left(B_{\mu \nu}, \lambda_{1}, \lambda_{2}, \phi\right)$. A two form with self-dual curvature $H_{3}=d B_{2}$, two fermions and a real scalar.

- Vector multiplets with field content $\left(A_{\mu}, \hat{\lambda}_{1}, \hat{\lambda}_{2}\right)$, a six-dimensional vector and two fermions.

- Hypermultiplets with field content $\left(\varphi_{1}, \varphi_{2}, \psi_{1}, \psi_{2}\right)$, two scalars and two fermions.

- Linear multiplets with field content $(\vec{\pi}, c, \tilde{\xi})$ an $S U(2)$ triplet and a singlet, together with a fermion.

These field theories have a tensor branch when the scalar $\phi$ gets a non-zero VEV. In this case, the $\mathrm{SU}(2)_{R}$ symmetry is preserved. On the other hand, when the scalars inside the hyper or the linear multiplet get VEVs, we explore the Higgs branch breaking the R-symmetry. In what follows we will be concerned with the tensor branch only.

To reproduce the Lorentz and R-symmetry mentioned above, the authors of [109] distributed D6, NS5, and D8 branes according to Table 3.1.

There are some key differences with Hanany-Witten set-ups in lower dimensions,

- The dimension of the field theory on the NS5-branes is the same as that on the bounded D6-branes. The non-decoupling of the five-branes dynamics adds the dynamical tensor multiplets to the field theories. These are absent in lower dimensional set-ups. 


\begin{tabular}{ccccccccccc} 
& $t$ & $x_{1}$ & $x_{2}$ & $x_{3}$ & $x_{4}$ & $x_{5}$ & $x_{6}$ & $x_{7}$ & $x_{8}$ & $x_{9}$ \\
\hline \hline NS5 & $\bullet$ & $\bullet$ & $\bullet$ & $\bullet$ & $\bullet$ & $\bullet$ & $\cdot$ & $\bullet$ & $\cdot$ & $\cdot$ \\
\hline \hline D6 & $\bullet$ & $\bullet$ & $\bullet$ & $\bullet$ & $\bullet$ & $\bullet$ & $\bullet$ & $\bullet$ & $\cdot$ & $\cdot$ \\
\hline D8 & $\bullet$ & $\bullet$ & $\bullet$ & $\bullet$ & $\bullet$ & $\bullet$ & $\bullet$ & $\bullet$ & $\bullet$ & $\bullet$
\end{tabular}

Table 3.1: The generic brane set-ups. All the branes are extended on the Minkowski $R^{1,5}$ directions. The D6-branes also extend over $x_{6}$ where they have finite size extension between NS5-branes. The D8-branes also extend along the $x_{7}, x_{8}$ and $x_{9}$ directions, preserving the $S O(3)_{R}$ symmetry.

- The bending of the NS5-branes due to other $p$-branes ending on them leads to a Laplace equation in $6-p$ dimensions. In this case, where $p=6$, there is no bending and the field content is always such that anomalies are canceled, namely

$$
N_{D 6, R}+N_{D 6, L}+N_{D 8}=2 N_{D 6, c}
$$

being $N_{D 6, R / L}$ the number of sixbranes to the right/left of a given stack with $N_{D 6, c}$ branes.

- We can consider D2-branes on $\left(t, x_{1}, x_{6}\right)$ that end on the NS5-branes. These branes represent one-dimensional magnetically-charged defects identified with the instantonic strings charged under the self-dual $H_{3}$.

- When the system is in the tensor branch (the difference between the scalars in different tensor multiplets $\left\langle\phi_{i}-\phi_{i-1}\right\rangle$ is non-zero) the instantonic strings are massive and the field theory can be described by an anomaly-free quiver. When $\left\langle\phi_{i}-\phi_{i-1}\right\rangle \rightarrow 0$, the theory is proposed 107 to flow to a strongly-coupled six-dimensional CFT with $(1,0)$ supersymmetry. These are the theories that we study in this paper.

\subsection{The dual $\mathrm{AdS}_{7}$ vacua}

Let us now discuss the holographic description of the SCFTs that appear when we move to the origin of the tensor branch. This description was developed in a set of papers, most notably 36 43]. We adopt the notation of [43].

The six-dimensional SCFTs have $\mathrm{SO}(2,6) \times \mathrm{SU}(2)_{R}$ bosonic symmetries, see for example [110. They are realised as the isometries of a massive type IIA vacuum of the form 


$$
\begin{aligned}
& \mathrm{d} s^{2}=f_{1}(z) \mathrm{d} s_{A d S_{7}}^{2}+f_{2}(z) \mathrm{d} z^{2}+f_{3}(z) \mathrm{d} \Omega_{2}^{2}(\chi, \xi), \\
& B_{2}=f_{4}(z) \operatorname{Vol}_{\Omega_{2}}, \quad F_{2}=f_{5}(z) \operatorname{Vol}_{\Omega_{2}}, \quad e^{\phi}=f_{6}(z), \quad F_{0}=F_{0}(z),
\end{aligned}
$$

where we have defined $\mathrm{d} \Omega_{2}^{2}(\chi, \xi)=\mathrm{d} \chi^{2}+\sin ^{2} \chi \mathrm{d} \xi^{2}$ and $\operatorname{Vol}_{\Omega_{2}}=\sin \chi \mathrm{d} \chi \wedge \mathrm{d} \xi$.

If we impose that $\mathcal{N}=(1,0)$ supersymmetry is preserved by the vacuum, we need the functions $f_{i}(z)$ to satisfy some first-order and nonlinear differential equations. Those are the Bogomol'nyi-Prasad-Sommerfield (BPS) equations, which are solved if the functions $f_{i}(z)$ in (3.3) are all defined in terms of a function $\alpha(z)$ and its derivatives [37, 43], as

$$
\begin{aligned}
& f_{1}(z)=8 \sqrt{2} \pi \sqrt{-\frac{\alpha}{\alpha^{\prime \prime}}}, \quad f_{2}(z)=\sqrt{2} \pi \sqrt{-\frac{\alpha^{\prime \prime}}{\alpha}}, \quad f_{3}(z)=\sqrt{2} \pi \sqrt{-\frac{\alpha^{\prime \prime}}{\alpha}}\left(\frac{\alpha^{2}}{\alpha^{\prime 2}-2 \alpha \alpha^{\prime \prime}}\right) \\
& f_{4}(z)=\pi\left(-z+\frac{\alpha \alpha^{\prime}}{\alpha^{\prime 2}-2 \alpha \alpha^{\prime \prime}}\right), \quad f_{5}(z)=\left(\frac{\alpha^{\prime \prime}}{162 \pi^{2}}+\frac{\pi F_{0} \alpha \alpha^{\prime}}{\alpha^{\prime 2}-2 \alpha \alpha^{\prime \prime}}\right) \\
& f_{6}(z)=2^{\frac{5}{4}} \pi^{\frac{5}{2}} 3^{4} \frac{\left(-\alpha / \alpha^{\prime \prime}\right)^{\frac{3}{4}}}{\sqrt{\alpha^{\prime 2}-2 \alpha \alpha^{\prime \prime}}} .
\end{aligned}
$$

Where $\alpha(z)$ has to satisfy the differential equation

$$
\alpha^{\prime \prime \prime}=-162 \pi^{3} F_{0}
$$

The function $\alpha(z)$ must be piece-wise continuous, which implies that $F_{0}$ is piece-wise constant and may be discontinuous. The internal space $\mathcal{M}^{3}=\left(z, \Omega_{2}\right)$ is a two-sphere fibered over the $z$-interval. The warp factor $f_{3}(z)$ must vanish at the beginning and at the end of the $z$-interval (i.e. $z=0$ and $z=z_{f}$, by convention), in such a way that the two-sphere shrinks smoothly at those points.

For a piece-wise constant and possibly discontinuous $F_{0}(z)$, the general solution to eq. 3.5 in each interval of constant $F_{0}$ is

$$
\alpha(z)=a_{0}+a_{1} z+\frac{a_{2}}{2} z^{2}-\frac{162 \pi^{3} F_{0}}{6} z^{3} .
$$

Since $\alpha(z)$ is piece-wise continuous, a polynomial solution like the one above should be proposed for each interval $\left[z_{i}, z_{i+1}\right]$; each such interval should exhibit its own parameters 
$\left\{a_{i}, F_{0}\right\}$. Imposing that the two-sphere shrinks smoothly at $z=0$ and $z=z_{f}$ implies that $\alpha(0)=\alpha\left(z_{f}\right)=0$. Before we discuss a generic solution, let us find general expressions for the D-brane charges associated to the vacua in (3.3).

\section{Page charges}

To that end, we consider the non-gauge-invariant but localized, conserved and quantized Page charges,

$$
Q_{D p}=\frac{1}{(2 \pi)^{7-p} g_{s}\left(\alpha^{\prime}\right)^{\frac{(7-p)}{2}}} \int \hat{F}_{8-p}, \quad Q_{N S 5}=\frac{1}{4 \pi^{2} g_{s}^{2} \alpha^{\prime}} \int H_{3}
$$

where $\hat{F}=e^{-B_{2}} \wedge F$ is the Page flux. In what follows we set $g_{s}=\alpha^{\prime}=1$. Using that $\alpha(0)=\alpha\left(z_{f}\right)=0$ we find the NS5-brane charge,

$$
Q_{N S 5}=\frac{1}{4 \pi^{2}} \int_{z, \Omega_{2}} \partial_{z} f_{4}=\frac{1}{\pi} \int_{z=0}^{z=z_{f}} \partial_{z} f_{4}=\frac{f_{4}\left(z_{f}\right)-f_{4}(0)}{\pi}=-z_{f} .
$$

Up to an orientation-related sign, the size of the $z$-interval equals the number of fivebranes. Hence we need to choose $z_{f}$ to be a positive integer. We shall take $Q_{N S 5}=z_{f}=N_{5}$ in what follows.

Calculating the charge of D6-branes, we find

$$
Q_{D 6}=\frac{1}{2 \pi} \int_{(\chi, \xi)} F_{2}-F_{0} B_{2}=\left[\frac{\alpha^{\prime \prime}+162 \pi^{3} F_{0} z}{81 \pi^{2}}\right]=\frac{\alpha^{\prime \prime}-z \alpha^{\prime \prime \prime}}{81 \pi^{2}}
$$

This charge gives the charge of D6-branes but includes, also, the charge of D6-branes induced onto the D8-branes. To avoid this 'over-counting' note that we may perform a large gauge transformation in any interval $[k, k+1]$ such that

$$
\hat{B}_{2} \rightarrow B_{2}+k \pi \mathrm{d} \Omega_{2}
$$

We then find that in the interval $[k, k+1]$ the Page charge reads

$$
Q_{D 6}=\frac{1}{2 \pi} \int_{S^{2}} F_{2}-F_{0} \hat{B}_{2}=\frac{1}{2 \pi} \times \frac{\alpha^{\prime \prime}-\alpha^{\prime \prime \prime}(z-k)}{162 \pi^{2}} \times 4 \pi
$$

Using that on the $[k, k+1]$ interval the function $\alpha^{\prime \prime}(z)=-81 \pi^{2}\left[N_{k}+\left(N_{k+1}-N_{k}\right)(z-k)\right]$, we find that 


$$
N_{D 6}=\frac{1}{2 \pi} \times \frac{\alpha^{\prime \prime}-\alpha^{\prime \prime \prime}(z-k)}{162 \pi^{2}} \times 4 \pi=-N_{k}
$$

The sign can be attributed to a choice of orientation for the two-sphere. The expression above indicates that in the $[k, k+1]$ interval, there are $N_{k}$ D6-branes. In other words, we performed a large gauge transformation to subtract the D6-charge induced onto the D8branes in eq. 3.9), in order to have a clear view of the number of D6-branes in their stacks.

We thus find that the number of the distinct D6-branes (and not of the ones in bound states with D8-branes) in the associated Hanany-Witten set-up is given by

$$
N_{D 6}=-\frac{1}{81 \pi^{2}} \int_{0}^{z_{f}} \alpha^{\prime \prime}(z) \mathrm{d} z
$$

This can be verified by explicitly performing this integral for a generic function $\alpha^{\prime \prime}(z)$, observing that it counts the sum of the ranks of the gauge groups (see eq. 3.29 ) for an example of a function $\alpha(z)$ for a generic quiver with four nodes and four flavour groups). On each interval $[k, k+1]$ this gives

$$
-\frac{1}{81 \pi^{2}} \int_{k}^{k+1} \alpha^{\prime \prime} d z=-\int_{k}^{k+1}\left[N_{k}+\left(N_{k+1}-N_{k}\right)(z-k)\right] d z=\frac{N_{k}+N_{k+1}}{2} .
$$

Summing over all the intervals (using that $N_{0}=N_{P+1}=0$ ), gives the total quantity

$$
N_{D 6}=\sum_{k=0}^{P} \frac{N_{k}+N_{k+1}}{2}=N_{1}+N_{2}+\ldots+N_{P}
$$

Considering the Page charge $Q_{D 8}=N_{D 8}=2 \pi \int F_{0}$ and eq. 3.5. , the number of D8branes in any given Hanany-Witten set-up reads

$$
N_{D 8}=\frac{1}{81 \pi^{2}}\left[\alpha^{\prime \prime \prime}(0)-\alpha^{\prime \prime \prime}\left(z_{f}\right)\right]
$$

In other words, the jumps in $\alpha^{\prime \prime \prime}(z)$ (coming from a piece-wise continuous function $\alpha(z)$ ) counts D8-branes across any interval, in accordance with eq.(3.5). Adding these jumps leads to the total number of D8-branes in a given set-up, i.e. eq.3.16.

These expressions are analogous to those derived in [111], for the case of Hanany-Witten set-ups associated with four-dimensional $\mathcal{N}=2$ SCFTs. In Section 2.2 we test the new expressions in eqs. $3.13,3.16$ on some examples. 


\section{Entanglement entropy}

Klebanov, Kutasov and Murugan [112] studied entanglement entropy (EE) in gravity vacua dual to confining large $\mathrm{N}_{c}$ gauge theories, generalizing the Ryu-Takayanagi conjecture 113 to non-conformal theories. They argued that, for this kind of theories, the volume of the $8-d$ compact dimensions and dilaton of a $\mathrm{AdS}_{d+2}$ vacuum are in general not constant and, hence, suggested that the EE in these cases is given by

$$
S_{E E}=\frac{1}{4 G_{N}} \int_{\gamma} \mathrm{d}^{8} \sigma e^{-2 \phi} \sqrt{\operatorname{det} g_{8, i n d}}
$$

where $G_{N}=8 \pi^{6} g_{s}^{2} \alpha^{4}=8 \pi^{6}$ is the ten-dimensional Newton constant, $\gamma$ is all surfaces with a common boundary with the entangling surface and $g_{8, \text { ind }}$ is the induced string frame metric on $\gamma$.

As an entangling surface they considered a strip of length $L_{E E}$ and, hence, the entanglement entropy $S_{E E}$ for a rectangular region of length $L_{E E}$ is found by minimizing an eight-manifold at the infinity of the AdS-radial direction. In this problem, there are two local minima of the action (3.17), given a particular $L_{E E}$ while, also, a regularization is needed, analogously to what happens when calculating Wilson loops. The first minimum reflects a disconnected surface, which consists of two regions which are separated by a distance $L_{E E}$. The second is a connected surface, in which the two regions are connected by a tube whose width depends on $L_{E E}$. EE is generally divergent in the UV but subtracting between its connected and disconnected phases we get a finite result.

For our kind of vacua, the treatment is the same as with [112,114]. That is, we consider an eight-manifold set in a gauge where its worldvolume coordinates equal distinctly spacetime dimensions as

$$
\Sigma_{8}=\left[x_{1}, x_{2}, x_{3}, x_{4}, x_{5}, z, \chi, \xi\right], \quad R=R\left(x_{1}\right)
$$

where $R$ is the AdS-radial coordinate, while we use Poincaré coordinates for the $\mathrm{AdS}_{7}$ space to acquire its induced metric as

$$
\mathrm{d} s_{8, \text { ind }}^{2}=f_{1}\left[R^{2} \mathrm{~d} \vec{x}_{4}^{2}+\mathrm{d} x_{1}^{2}\left(R^{2}+\frac{R^{\prime 2}}{R^{2}}\right)\right]+f_{2} \mathrm{~d} z^{2}+f_{3}\left(\mathrm{~d} \chi^{2}+\sin ^{2} \chi \mathrm{d} \xi^{2}\right) .
$$

Then the entanglement entropy is

$$
S_{E E}=\frac{128 V_{4}}{6561 G_{N}}\left(\int_{0}^{z_{f}} \alpha^{\prime \prime}(z) \alpha(z) \mathrm{d} z\right) \int \mathrm{d} x_{1} R^{5} \sqrt{1+\frac{R^{\prime 2}}{R^{4}}}, \quad V_{4}=\int \mathrm{d} x_{2} \mathrm{~d} x_{3} \mathrm{~d} x_{4} \mathrm{~d} x_{5} .
$$


Following the formalism of [114, we find the regularized entanglement entropy

$$
\begin{aligned}
S_{E E}^{r e g} & =\frac{V_{4}}{2 G_{N}}\left[\int_{1}^{\infty} \mathrm{d} y\left(\frac{y^{8}}{\sqrt{y^{10}-1}}-y^{3}\right)\right] \mathcal{N} R_{0}^{4}=\mu_{1} \mathcal{N} R_{0}^{4}, \\
\mathcal{N} & =-\frac{512}{6561} \int_{0}^{z_{f}} \alpha(z) \alpha^{\prime \prime}(z) \mathrm{d} z
\end{aligned}
$$

and the separation between the two regions

$$
L_{E E}=\left[2 \int_{1}^{\infty} \frac{\mathrm{d} y}{\sqrt{y^{4}\left(y^{10}-1\right)}}\right] \frac{1}{R_{0}}=\frac{\mu_{2}}{R_{0}} .
$$

Substituting $L_{E E}$ into $S_{E E}^{r e g}$ we get the final expression

$$
S_{E E}^{r e g}=\mathcal{N}\left(\frac{\mu_{1} \mu_{2}^{4}}{L^{4}}\right)
$$

The factors $\mu_{1} \mu_{2}^{4}$ are common to all six-dimensional CFTs. The power $L^{-4}$ is the only possible one given conformality and the dimension of the CFT. All the information about the particular CFT in consideration is in the factor $\mathcal{N} \sim \int \alpha \alpha^{\prime \prime}$.

\section{Central charge}

A quantity closely related to that of entanglement entropy and of great importance in CFT is the central charge. To illustrate its holographic expression, we follow the formalism developed in 112, 114 and in 115].

In [112, a generic metric on a type II string theory vacuum was considered, assumingly dual to a $(d+1)$-dimensional CFT, which, in string frame, reads

$$
\mathrm{d} s^{2}=a(r)\left(\mathrm{d} x_{1, d}^{2}+b(r) \mathrm{d} r\right)+g_{i j} \mathrm{~d} \theta^{i} \mathrm{~d} \theta^{j}
$$

The $(d+1)$-dimensional Minkowski space is parametrized by $\mathrm{d} x_{1, d}^{2}, g_{i j}$ is the metric field on the internal manifold and $a, b$ are functions of the AdS radial coordinate $r$, while the vacuum may also be supported by a dilaton field. For this type of background, the holographic central charge is defined by the quantities 


$$
H=e^{-4 \Phi} V_{\text {int }} a^{d}, \quad V_{\text {int }}=\int \mathrm{d} \vec{\theta} \sqrt{\operatorname{det} g_{i j}}
$$

and it is proportional to the volume of the internal space.

Nonetheless, in our kind on vacua the functions $a, b$ (and the dilaton) depend on the

coordinates $\vec{\theta}$ of the internal manifold. In this case, it was proposed in 114 that the above defining quantities should be modified as

$$
\hat{H}=\hat{V}_{\text {int }}^{2}, \quad \hat{V}_{\text {int }}=\int \mathrm{d} \vec{\theta} \sqrt{e^{-4 \Phi} a^{d} \operatorname{det} g_{i j}},
$$

since now $a, \Phi$ are generally dependent on $\vec{\theta}$. Then, the central charge of the $(d+1)$ dimensional CFT is defined to be

$$
c=\frac{d^{d} b^{\frac{d}{2}} \hat{H}^{\frac{2 d+1}{2}}}{G_{N}\left(\hat{H}^{\prime}\right)^{d}},
$$

where $G_{N}=\left(l_{P}\right)^{D-2}$ is the Newton constant in $D$ spacetime dimensions, with $l_{P}$ the Planck length.

Plugging our family of vacua (3.3) into the above expression, as was first done in [61, we acquire the formula

$$
c=-\frac{1}{16 G_{N}}\left(\frac{2}{3}\right)^{8} \int_{0}^{z_{f}} \alpha(z) \alpha^{\prime \prime}(z) \mathrm{d} z
$$

which is the holographic central charge for the kind of CFTs we consider in this chapter. Notice, here, that this charge is proportional to the entanglement entropy in (3.18), up to some numerical factor, which is exactly as it should be since both quantities measure the number of degrees of freedom.

\subsection{The quiver gauge theory}

Before we sail off into the main subject of integrability, let us illustrate the duality between the $\mathrm{AdS}_{7}$ vacua of the form (3.3) and the six-dimensional field theory. Those vacua are parametrized by $\alpha(z)$ and its derivatives and each choice for that function corresponds to a distinct vacuum and, hence, a particular quiver theory.

For clarity, let us work the other way around and reconstruct the $\mathrm{AdS}_{7}$ vacuum from a specific quiver gauge theory. To that end, we consider a quiver structure with gauge and flavor 
groups bound together through bifundamental matter, satisfying condition (3.2) and, hence, being non-anomalous. Moreover, we define the function $R(z)$, a piecewise continuous linear function such that at $z=j$ (with $j$ being a positive integer number) the value $R(j)=N_{j}$ is the rank of the $j$-th gauge group. It was shown in [43] that this rank-function must be convex to satisfy the anomaly cancellation condition in eq. (3.2). $R(z)$ is not necessary to understand or work out the holographic duality; it is merely a matter of convenience, a intermediate step that clarifies the connection between the rank of the quiver gauge groups and the number of D6-branes in string theory.

Therefore, noticing the expression for the number of D6-branes in (3.13), we define the rank-function to be

$$
R(z)=-\frac{1}{81 \pi^{2}} \alpha^{\prime \prime}(z)
$$

Choosing a particular rank-function and considering this identification, equation (3.26), we may determine the function $\alpha(z)$ by imposing boundary conditions and continuity of $\alpha$ and $\alpha^{\prime}$.

In order to understand the ways of holography and comprehend the formalism presented above, we now give a couple of examples. For a larger variety of those, 61 should be consulted]

A simple example Consider the Hanany-Witten set-up, quiver and Rank function $R(z)$ in Figures 3.13 .3$.

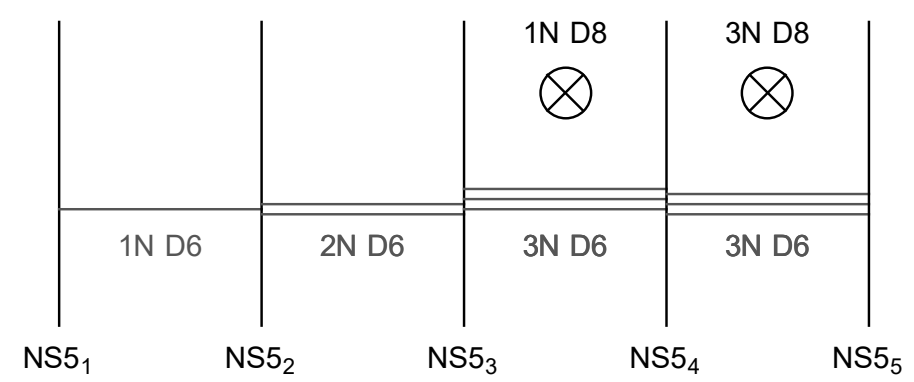

Figure 3.1: The Hanany-Witten set-up for the field theory. The vertical lines denote individual Neveu-Schwarz branes extended on the $\left(x_{4}, x_{5}\right)$ space. The horizontal ones D6-branes, that extend on $x_{6}$, in between fivebranes. The crossed-circles represent D8-branes, that extend on the $\left(x_{7}, x_{8}, x_{9}\right)$ directions. All the branes share the Minkowski directions. This realises the isometries $\mathrm{SO}(1,5) \times \mathrm{SO}(3)$.

\footnotetext{
${ }^{1}$ In order for the supergravity vacuum to capture faithfully the SCFT dynamics one should work with long linear quivers, with large ranks. In this sense, the examples of 61 are trustable, while our examples in this section are not but should be taken as illustrative of the procedure.
} 


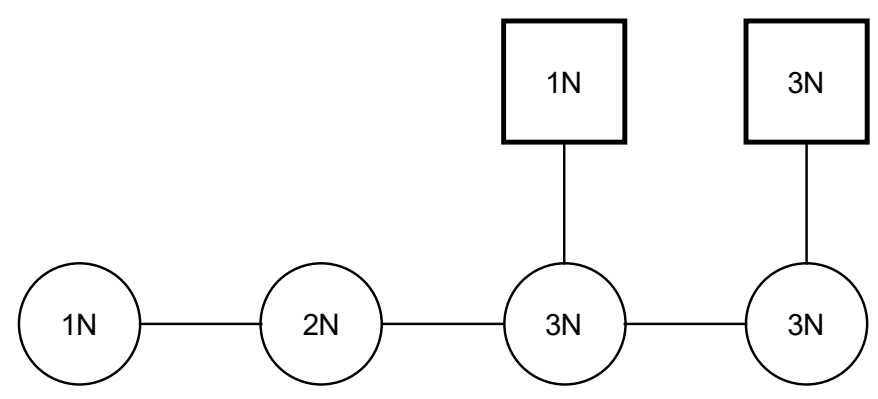

Figure 3.2: The quiver corresponding to the Hanany-Witten set-up above. The circle nodes are gauge groups, individual gauge theories, and the square nodes are flavor symmetries.

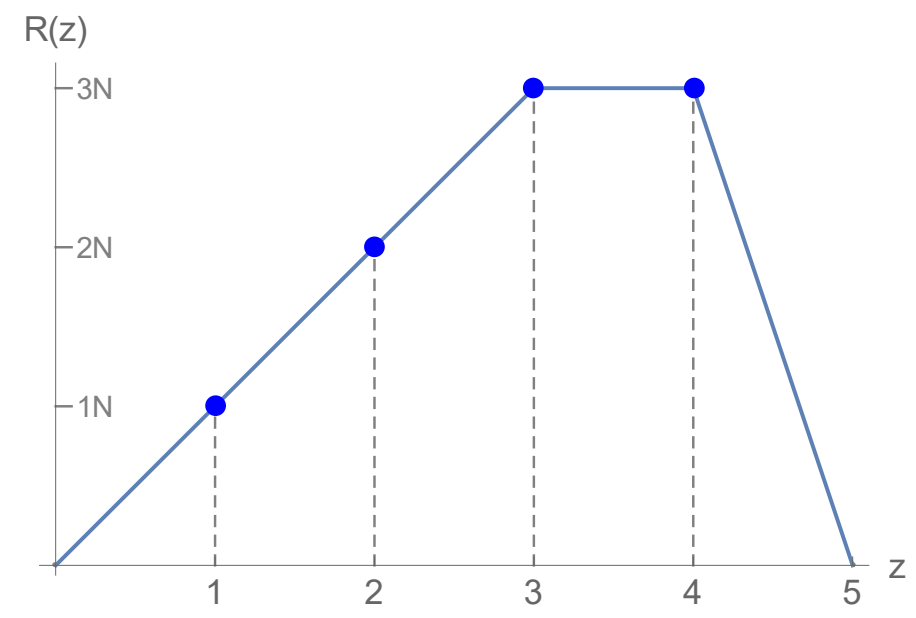

Figure 3.3: The rank-function $R(z)$ corresponding to the field theory, defined by the ranks of the gauge groups on the quiver structure.

In this example, the rank function and the function $\alpha^{\prime \prime}(z)$ are given by,

$$
R(z)=-\frac{1}{81 \pi^{2}} \alpha^{\prime \prime}(z)=N \begin{cases}z & 0 \leq z \leq 1 \\ (z-1)+1 & 1 \leq z \leq 2 \\ (z-2)+2 & 2 \leq z \leq 3 \\ 3 & 3 \leq z \leq 4 \\ 3-3(z-4) & 4 \leq z \leq 5\end{cases}
$$


This implies that the generic function $\alpha(z)$ for this example is,

$$
\alpha(z)=-81 \pi^{2} N \begin{cases}a_{0}+a_{1} z+\frac{z^{3}}{6} & 0 \leq z \leq 1 \\ b_{0}+b_{1}(z-1)+\frac{1}{2}(z-1)^{2}+\frac{1}{6}(z-1)^{3} & 1 \leq z \leq 2 \\ c_{0}+c_{1}(z-2)+2 \frac{(z-2)^{2}}{2}+\frac{1}{6}(z-2)^{3} & 2 \leq z \leq 3 \\ d_{0}+d_{1}(z-3)+3 \frac{(z-3)^{2}}{2} & 3 \leq z \leq 4 \\ p_{0}+p_{1}(z-4)+3 \frac{(z-4)^{2}}{2}-3 \frac{(z-4)^{3}}{6} & 4 \leq z \leq 5 .\end{cases}
$$

To determine the ten integration constants, we need to impose:

- That $\alpha(0)=\alpha(5)=0$. This is to have an internal space that shrinks smoothly at the beginning and end of the $z$-interval. These conditions imply

$$
a_{0}=0, \quad p_{0}+p_{1}+\frac{3}{2}-\frac{3}{6}=0
$$

- That the function $\alpha(z)$ is continuous, this implies the equations

$$
a_{1}+\frac{1}{6}=b_{0}, \quad b_{0}+b_{1}+\frac{1}{2}+\frac{1}{6}=c_{0}, \quad c_{0}+c_{1}+1+\frac{1}{6}=d_{0}, \quad d_{0}+d_{1}+\frac{3}{2}=p_{0} .
$$

- That the function $\alpha^{\prime}(z)$ is continuous. This implies

$$
a_{1}+\frac{1}{2}=b_{1}, \quad b_{1}+1+\frac{1}{2}=c_{1}, \quad c_{1}+2+\frac{1}{2}=d_{1}, \quad d_{1}+3=p_{1} .
$$

Solving these equations we find,

$$
\begin{aligned}
& a_{0}=0, \quad-5 a_{1}=19, \quad-30 b_{0}=109, \quad-10 b_{1}=33, \quad-15 c_{0}=94, \quad-5 c_{1}=9, \\
& -10 d_{0}=69, \quad 10 d_{1}=7, \quad-10 p_{0}=47, \quad 10 p_{1}=37 .
\end{aligned}
$$

which define completely the function $\alpha(z)$.

As a confirmation, we may apply $\alpha(z)$ to acquire the number of NS, D6 and D8-branes. Indeed, using equations (3.8), 3.13) and 3.16 we find

$$
\begin{aligned}
& N_{N S 5}=z_{f}=5, \quad N_{D 8}=\frac{1}{81 \pi^{2}}\left[\alpha^{\prime \prime \prime}(0)-\alpha^{\prime \prime \prime}\left(z_{f}\right)\right]=4 N \\
& N_{D 6}=-\frac{1}{81 \pi^{2}} \int_{0}^{z_{f}} \alpha^{\prime \prime}(z) \mathrm{d} z=9 N .
\end{aligned}
$$


which coincide with the numbers we count from the Hanany-Witten set-up in Figure 3.1. Let us now study a more generic example.

A more generic example Consider the field theory represented by the Hanany-Witten set-up in Figure 3.4 or equivalently, the quiver in Figure 3.5.

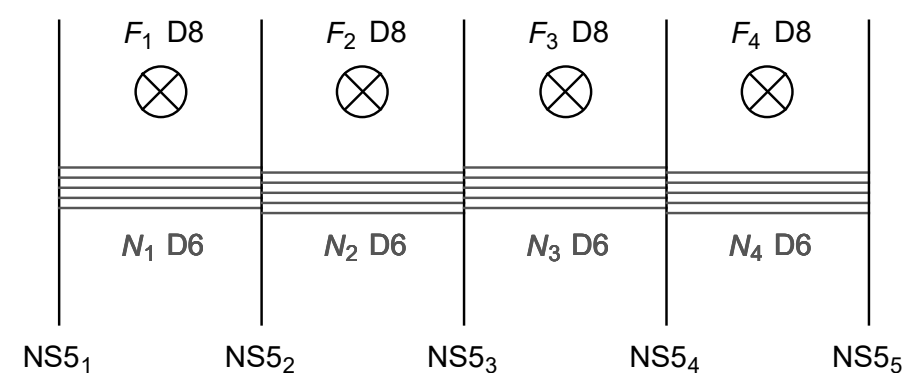

Figure 3.4: The Hanany-Witten set-up corresponding to the generic field theory studied here.

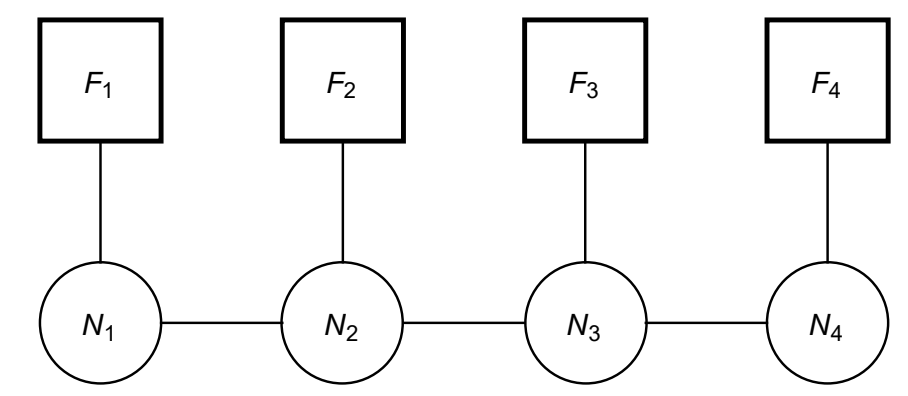

Figure 3.5: The quiver corresponding to the generic field theory studied here.

Notice that, while in the previous example we considered specific numbers for the gauge or flavor groups, we now have set the ranks to be general. Thus, while the previous quiver theory implicitly satisfied condition (3.2) (by construction, for the gauge anomalies to cancel), we now need to impose

$$
\begin{aligned}
& 2 N_{1}-N_{2}=F_{1}, \quad 2 N_{2}-N_{1}-N_{3}=F_{2}, \\
& 2 N_{3}-N_{2}-N_{4}=F_{3}, \quad 2 N_{4}-N_{3}=F_{4} .
\end{aligned}
$$


We construct the rank-function

$$
R(z)= \begin{cases}N_{1} z & 0 \leq z \leq 1 \\ \left(N_{2}-N_{1}\right)(z-1)+N_{1} & 1 \leq z \leq 2 \\ \left(N_{3}-N_{2}\right)(z-2)+N_{2} & 2 \leq z \leq 3 \\ \left(N_{4}-N_{3}\right)(z-3)+N_{3} & 3 \leq z \leq 4 \\ -N_{4}(z-4)+N_{4} & 4 \leq z \leq 5\end{cases}
$$

The function $\alpha(z)$ reads,

$$
\alpha(z)=-81 \pi^{2} \begin{cases}a_{0}+a_{1} z+N_{1} \frac{z^{3}}{6} & 0 \leq z \leq 1 \\ b_{0}+b_{1}(z-1)+N_{1} \frac{(z-1)^{2}}{2}+\left(N_{2}-N_{1}\right) \frac{(z-1)^{3}}{6} & 1 \leq z \leq 2 \\ c_{0}+c_{1}(z-2)+N_{2} \frac{(z-2)^{2}}{2}+\left(N_{3}-N_{2}\right) \frac{(z-2)^{3}}{6} & 2 \leq z \leq 3 \\ d_{0}+d_{1}(z-3)+N_{3} \frac{(z-3)^{2}}{2}+\left(N_{4}-N_{3}\right) \frac{(z-3)^{3}}{6} & 3 \leq z \leq 4 \\ p_{0}+p_{1}(z-4)+N_{4} \frac{(z-4)^{2}}{2}-N_{4} \frac{(z-4)^{3}}{6} & 4 \leq z \leq 5 .\end{cases}
$$

Again, we determine the ten coefficients by imposing that $\alpha(0)=\alpha(5)=0$ and the continuity

of $\alpha(z)$ and $\alpha^{\prime}(z)$. The resolution of the algebraic system is straightforward and, acquiring the function $\alpha(z)$, we may calculate the number of D-branes as

$$
\begin{aligned}
& N_{N S 5}=z_{f}=5, \quad N_{D 8}=\frac{1}{81 \pi^{2}}\left[\alpha^{\prime \prime \prime}(0)-\alpha^{\prime \prime \prime}(5)\right]=N_{1}+N_{4}=F_{1}+F_{2}+F_{3}+F_{4}, \\
& N_{D 6}=-\frac{1}{81 \pi^{2}} \int_{0}^{z_{f}} \alpha^{\prime \prime} \mathrm{d} z=N_{1}+N_{2}+N_{3}+N_{4},
\end{aligned}
$$

which, of course, coincide with the Hanany-Witten set-up in Figure 3.4.

\section{Non-integrability on the generic $\mathrm{AdS}_{7}$ vacua}

We now move on into the main subject of this chapter, which is the study of classical integrability of these $\mathrm{AdS}_{7}$ vacua and, subsequently, of their dual six-dimensional SCFTs. We employ our tools of analytic non-integrability just as we did in Chapters 1 and 2 , by exploring the dynamics of the $\sigma$-model on those vacua and searching for dynamical subsectors of the bosonic string that exhibit non-integrability. We do this because, as stressed on the previous chapters, an integrable theory has all of its dynamical sectors integrable, which means that even a single sector with non-integrable behavior is enough to declare a supergravity vacuum 
(or a whole family of those, in our case) as non-integrable.

Of course, whenever the methods of analytic non-integrability fail to spot the homonymous property on a vacuum, that does not necessarily imply integrability. In other words, even if those methods do not show non-integrability and give way to the possibility of integrability, this possibility is certainly not a certainty. Integrability may or may not be there. As we show below, the $\mathrm{AdS}_{7}$ vacua defined by a generic function $\alpha(z)$ are non-integrable, while there is one and only one vacuum (for a special choice of this function) where nonintegrability recedes and the possibility of integrability pops up. In that particular case, the vacuum is indeed integrable and we prove this in the next section.

We begin by expanding the Neveu-Schwarz sector of the background in eq.(3.3) in global coordinates for $\mathrm{AdS}_{7}$ as

$$
\begin{aligned}
d s^{2}= & f_{1}(z)\left[-\mathrm{d} t^{2} \cosh \rho+\mathrm{d} \rho^{2}+\sinh ^{2} \rho\left(\mathrm{d} \varphi^{2}+\cos ^{2} \varphi \mathrm{d} \theta^{2}+\sin ^{2} \varphi \mathrm{d} \Omega_{3}\right)\right]+f_{2}(z) \mathrm{d} z^{2} \\
& +f_{3}(z)\left(\mathrm{d} \chi^{2}+\sin ^{2} \chi \mathrm{d} \xi^{2}\right), \quad B_{2}=f_{4}(z) \sin \chi \mathrm{d} \chi \wedge \mathrm{d} \xi .
\end{aligned}
$$

Following the logic of the previous chapters, we desire dynamics that is supported by secondorder ordinary differential equations of motion. Therefore, once again, we propose a string embedding of the form

$$
t=t(\tau), \quad \rho=\rho(\tau), \quad \varphi=\varphi(\tau), \quad \theta=\mu \sigma, \quad z=z(\tau), \quad \chi=\chi(\tau), \quad \xi=\kappa \sigma,
$$

where the integers $\kappa$ and $\mu$ indicate the number of times the bosonic string wraps around the $\xi$ and $\theta$-dimensions, respectively. The rest of the variables are set to zero.

We study the equations of motion of this soliton derived from the Polyakov action, supplemented by the Virasoro constraint,

$$
\begin{aligned}
& S_{P}=-\frac{1}{4 \pi \alpha^{\prime}} \int_{\Sigma} \mathrm{d}^{2} \sigma\left(\eta^{a b} G_{\mu \nu}+\epsilon^{a b} B_{\mu \nu}\right) \partial_{a} X^{\mu} \partial_{b} X^{\nu} \\
& T_{a b}=\partial_{a} X^{\mu} \partial_{b} X^{\nu} G_{\mu \nu}-\frac{1}{2} \eta_{a b} \eta^{c d} \partial_{c} X^{\mu} \partial_{d} X^{\nu} G_{\mu \nu}=0 .
\end{aligned}
$$

The equations of motion are 


$$
\begin{aligned}
f_{1}(z) \dot{t}= & \frac{E}{\cosh ^{2} \rho} \\
f_{1}(z) \ddot{\rho}= & -\frac{E^{2}}{f_{1}(z)} \frac{\sinh \rho}{\cosh ^{3} \rho}+f_{1}(z) \sinh \rho \cosh \rho\left(\dot{\varphi}^{2}-\mu^{2} \sin ^{2} \varphi\right)-f_{1}^{\prime}(z) \dot{\rho} \dot{z} \\
f_{1}(z) \ddot{\varphi}= & -f_{1}(z)\left(2 \frac{\cosh \rho}{\sinh \rho} \dot{\varphi} \dot{\rho}+\mu^{2} \cos \varphi \sin \varphi\right)-f_{1}^{\prime}(z) \dot{z} \dot{\varphi} \\
f_{3}(z) \ddot{\chi}= & \kappa f_{4}^{\prime}(z) \dot{z} \sin \chi-f_{3}^{\prime}(z) \dot{z} \dot{\chi}-\kappa^{2} f_{3}(z) \sin \chi \cos \chi \\
2 f_{2}(z) \ddot{z}= & f_{1}^{\prime}(z)\left(-\frac{E^{2}}{f_{1}(z)^{2} \cosh ^{2} \rho}+\dot{\rho}^{2}+\sinh ^{2} \rho\left(\dot{\varphi}^{2}-\mu^{2} \sin ^{2} \varphi\right)\right)-f_{2}^{\prime}(z) \dot{z}^{2} \\
& +f_{3}^{\prime}(z)\left(\dot{\chi}^{2}-\kappa^{2} \sin ^{2} \chi\right)-2 \kappa \dot{\chi} \sin \chi f_{4}^{\prime}(z) .
\end{aligned}
$$

where the dot indicates derivatives with respect to $\tau$ and the prime derivatives with respect to $z$. We have used the first equation above, to replace for $\dot{t}$ in the other four equations.

The Virasoro constraints for the string soliton are

$$
\begin{aligned}
& T_{\sigma \tau}=0, \quad T_{\tau \tau}=T_{\sigma \sigma}=0 \rightarrow \\
& f_{1}(z)\left(-\cosh ^{2} \rho \dot{t}^{2}+\dot{\rho}^{2}+\sinh ^{2} \rho\left(\dot{\varphi}^{2}+\mu^{2} \sin ^{2} \varphi\right)\right)+f_{2}(z) \dot{z}^{2}+f_{3}(z)\left(\dot{\chi}^{2}+\kappa^{2} \sin ^{2} \chi\right)=0 .
\end{aligned}
$$

This constraint holds regardless of the equations of motion and, thus, it is a primary constraint. The energy-momentum tensor is preserved on shell, $\nabla_{a} T^{a b}=0$, since $\partial_{\tau} T_{\tau \tau}=$ $\partial_{\sigma} T_{\sigma \sigma}=0$ on the equations of motion (1.17). Note, also, that the compliance of the worldsheet constraints with the equations of motion yield the consistency of our embedding.

We proceed by noticing that the invariant plane of motion with

$$
\ddot{\varphi}(\tau)=\dot{\varphi}(\tau)=\varphi(\tau)=\ddot{\chi}(\tau)=\dot{\chi}(\tau)=\chi(\tau)=\ddot{\rho}(\tau)=\dot{\rho}(\tau)=\rho(\tau)=0 .
$$

automatically solves the equations of motion in $(3.33)$, while it reduces the equation for $z(\tau)$ to 


$$
2 f_{2}(z) \ddot{z}=-\frac{f_{1}^{\prime}(z)}{f_{1}(z)^{2}} E^{2}-\dot{z}^{2} f_{2}^{\prime}(z)
$$

After inserting the expressions for the functions $f_{1}(z)$ and $f_{2}(z)$ in terms of $\alpha(z)$, the above equation reads

$$
\ddot{z}-\left(\frac{\alpha \alpha^{\prime \prime \prime}-\alpha^{\prime} \alpha^{\prime \prime}}{4 \alpha^{2}}\right)\left(\frac{\alpha}{\alpha^{\prime \prime}}\right)\left(\frac{E^{2}}{16 \pi^{2}}-\dot{z}^{2}\right)=0,
$$

which has a simple solution

$$
z_{s o l}(\tau)=\frac{E}{4 \pi} \tau
$$

This also solves the constraint in eq. 3.34 using the first of eqs. 3.33 for $\dot{t}$ and the expressions for $f_{1}(z)$ and $f_{2}(z)$.

Hence, we have a 'base solution' around which we perturb the other variables. This leads to the Normal Variational Equation (NVE) for the different coordinates and it is those fluctuations that we use to reveal the dynamics of the system.

\subsection{NVE for a spherical coordinate}

In spite of the fact that we have previously, in Chapter 1, used exclusively the radial dimension to study a NVE, it is now more suitable (for reasons explained in Appendix E) to handle one of the spherical coordinates of the $\mathrm{AdS}_{7}$ space.

Hence, we allow for small fluctuations $\varphi(\tau)=0+\epsilon f(\tau)$ over the equation of motion for $\ddot{\varphi}(\tau)$ in (3.33), into which we insert the simple solution $z_{\text {sol }}(\tau),(3.38)$, while we keep all other variables to zero, according to the base solution. The resulting NVE, at leading order of the small parameter $\epsilon$, reads

$$
\begin{aligned}
\ddot{f}(\tau)+\mathcal{B}(\tau) \dot{f}(\tau) & +\mathcal{A}(\tau) f(\tau)=0 \\
\mathcal{B}_{f}(\tau) & =\left.\frac{f_{1}^{\prime}(z)}{f_{1}(z)} \frac{E}{4 \pi}\right|_{z_{s o l}}=\left.\frac{E}{8 \pi}\left(\frac{\alpha^{\prime}}{\alpha}-\frac{\alpha^{\prime \prime \prime}}{\alpha^{\prime \prime}}\right)\right|_{z_{s o l}} \\
\mathcal{A}_{f}(\tau) & =\mu^{2}
\end{aligned}
$$

where the function $\alpha(z)$ - with $z=z(\tau)=z_{\text {sol }}(\tau)$ - is taken, of course, in its fully general 
form, (3.6). The analysis on the Liouville integrability of this equation is relegated to Appendix E and the final result is that it does not admit any Liouvillian solutions, therefore yielding a non-integrable dynamical system. That is, since we chose $\alpha(z)$ to be general (i.e. rendered by generic polynomial parameters $\left\{a_{i}, F_{0}\right\}$ ), the result is general too and yields the complete non-integrability of the whole family of these supergravity vacua. That is, if $\alpha(z)$ is a polynomial of the form 3.6 .

At this point, we make a simple observation: if the warp factor $f_{1}(z)$ is equal to a constant, then $\mathcal{B}_{f}=0$ and the above differential equation is that of a harmonic oscillator, which admits a Liouvillian solution of the form $f(\tau)=\exp (i \mu \tau)$. The exact same fact holds for the NVE of $\rho(\tau)$, together posing a hint for integrability. On the other hand, we only study a dynamical subsector of a particular soliton (and not the complete dynamics of all possible string configurations), which does not provide any real proof of classical integrability for this special vacuum. As a matter of fact, this observation is actually an expected result, considering that a constant $f_{1}(z)$ unwarps the $\mathrm{AdS}_{7}$ subspace from the internal $\mathcal{M}^{3}$ (and so fluctuations on the symmetric AdS, of variables $\rho$ and $\varphi$, are expected to be integrable).

Nonetheless, a hint is a hint and we should follow it through to see if there is any deeper truth underneath it. And in our case, finally, there is. This is the subject of the next section.

\section{$4 \quad$ Integrability on a special vacuum}

The analysis above strongly suggests that the case of a constant $\mathrm{AdS}_{7}$ warp factor is quite special. Considering the $\mathrm{AdS}_{7}$ warping by $f_{1}(z)$, this implies a background-defining function

$$
\alpha(z)=A \sin (\omega z)
$$

for which both functions $f_{1}(z)$ and $f_{2}(z)$ are constant. This solution does not fall within the class of solutions defined by $\alpha(z)$ in (3.6) and studied in Section 2.1. We will understand what this change in the definition of $\alpha(z)$ implies, momentarily, by the new form of the $F_{0}$ flux that it is produced.

When $\alpha(z)=A \sin (\omega z)$, the $z$-coordinate varies in the interval $0 \leq z \leq \frac{\pi}{\omega}$, where we choose $\omega=\frac{\pi}{N_{5}}$ with $N_{5}$ being a large integer. Plugging this into the full vacuum defined by (3.3) and (3.4), we get 


$$
\begin{aligned}
& \mathrm{d} s^{2}=\frac{\sqrt{2} \pi}{\omega}\left(8 \mathrm{~d} s_{A d S_{7}}^{2}+\omega^{2} \mathrm{~d} z^{2}+\left(\frac{\sin ^{2} \omega z}{1+\sin ^{2} \omega z}\right) \mathrm{d} \Omega_{2}\right), \\
& e^{-2 \phi}=e^{-2 \phi_{0}}\left(1+\sin ^{2} \omega z\right), \quad B_{2}=\pi\left(-z+\frac{\sin \omega z \cos \omega z}{\omega\left(1+\sin ^{2} \omega z\right)}\right) \mathrm{d} \Omega_{2}, \\
& F_{0}=\frac{A \omega^{3} \cos \omega z}{162 \pi^{3}}, \quad \quad F_{2}=-\frac{A \omega^{2}}{81 \pi^{2}}\left(\frac{\sin ^{3} \omega z}{1+\sin ^{2} \omega z}\right) \mathrm{d} \Omega_{2} .
\end{aligned}
$$

The expression for $F_{0}$ suggests that we have a continuous distribution of D8-branes, along the $z$-dimension. Indeed, in contrast with the examples in Section 2.1, $F_{0}$ in (3.41) is a continuous function, instead of a piece-wise constant and discontinuous one that reflects (through the associated Bianchi identity) localized D8-branes.

What makes the vacuum 3.41 special is the fact that $\mathrm{AdS}_{7}$ and $\mathcal{M}^{3}$ are now decoupled. The geometry becomes a direct product $\mathrm{AdS}_{7} \times \mathcal{M}^{3}$ and bosonic string theory factorizes into a $\sigma$-model on $\mathrm{AdS}_{7}$ times a $\sigma$-model on $\mathcal{M}^{3}$ coupled to a $B_{2}$ field. All this, up to the Virasoro constraint that glues together the total worldsheet theory, acting as a primary constraint on the target-space equations of motion. On the level of the action, we have

$$
\begin{aligned}
S_{P} & =S_{P}^{\mathrm{AdS}_{7}}+S_{P}^{\mathcal{M}^{3}} \\
& =-\frac{1}{4 \pi \alpha^{\prime}} \int_{\Sigma} d^{2} \sigma \eta^{a b} G_{\alpha \beta}^{\mathrm{AdS}_{7}} \partial_{a} X^{\alpha} \partial_{b} X^{\beta}-\frac{1}{4 \pi \alpha^{\prime}} \int_{\Sigma} d^{2} \sigma\left(\eta^{a b} G_{\mu \nu}^{\mathcal{M}^{3}}+\epsilon^{a b} B_{\mu \nu}^{\mathcal{M}^{3}}\right) \partial_{a} X^{\mu} \partial_{b} X^{\nu}
\end{aligned}
$$

where the Latin indices range over the worldsheet coordinates and the Greek indices over the target-space ones. In particular, $(\alpha, \beta)$ are in $\mathrm{AdS}_{7}$ and $(\mu, \nu)$ run over $z, \chi$ and $\xi$ of the internal space $\mathcal{M}^{3}$.

Now, since $\mathrm{AdS}_{7}$ is a symmetric space and, as such, should host integrable dynamics for the bosonic string, we bring our attention to subspace $\mathcal{M}^{3}$. We may approach the problem with the following logic. As already emphasized, integrability depends on the existence of a Lax connection on the cotangent bundle of the theory, while no standard recipe is provided to acquire such a construction. In fact, there is not even an a priori reason to decide whether such a connection does exist. Therefore, integrable systems are mainly obtained as structure-preserving deformations of known integrable theories, 27 30]. Having acknowledged that, we recall that $\mathcal{M}^{3}$ is isomorphic to $\mathrm{S}^{3}$, which may suggest that, if integrability is indeed present (and not just a hint of the calculation in the previous section), it could be the case that $\mathcal{M}^{3}$ is an integrable deformation of $S^{3}$, the latter being symmetric (as a geometry, 
without a $B_{2}$ field) and hosting integrable dynamics for the bosonic string. The familiar paradigm for such a scenario is the $\lambda$-deformation of the WZW model on $\mathrm{SU}(2) \cong \mathrm{S}^{3},[116$, and our chance here would be that the string $\sigma$-model on $\mathcal{M}^{3}$ is a $\lambda$-deformed WZW model on $\mathrm{S}^{3}$, for a particular value of the $\lambda$ parameter. This is indeed the case.

\section{$4.1 \mathcal{M}^{3}$ as a $\lambda$-deformation of $\mathrm{S}^{3}$}

As it turns out, the Neveu-Schwarz sector of the bosonic string on $\mathcal{M}^{3}$, for the vacuum (3.41), is exactly equal to a $\lambda$-deformed WZW model on $\mathrm{SU}(2)$. The $\lambda$-deformation is an integrable deformation of the WZW model proposed by Sfetsos in 28. The WZW model on a Lie group $G$ is given by an action

$$
S_{W Z W, k}=\frac{k}{2 \pi} \int_{\partial \mathcal{B}} \operatorname{Tr}\left[j_{a} j^{a}\right]+\frac{k}{6 \pi} \int_{\mathcal{B}} \epsilon_{a b c} \operatorname{Tr}\left[j^{a} j^{b} j^{c}\right]
$$

where $k \in \mathbb{Z}$ is the level of the model and $j_{a}$ is an algebra-valued current form on $G$. The first term is the action of the Principal Chiral Model (PCM), which may be realized as the metric-field part of the string $\sigma$-model on a group manifold. The second WZ-term may be realized as the dynamics generated by a $B_{2}$ field. Both the PCM and the WZW model on a Lie group $\mathrm{G}$ are integrable and their features and structure are introduced in Appendix $\mathrm{F}$.

The $\lambda$-model is practically the sum of the PCM and WZW model. Since both of those ingredient models are integrable, so is their combination. The level $k$ of the WZW model combines with the coupling parameter of the PCM and make up what we call the $\lambda$ parameter. The action of the $\lambda$-model on a Lie group $G$ then reads

$$
S_{\lambda}=S_{W Z W, k}+\frac{k}{\pi} \int_{\partial \mathcal{B}} \tilde{\mathrm{j}}_{+}^{A}\left(\lambda^{-1}-D^{T}\right)_{A B}^{-1} j_{-}^{B}
$$

where $j_{a}$ is a left-invariant current and $\tilde{\mathrm{j}}_{a}=\partial_{a} g g^{-1}$ is a right-invariant current. Notice that these currents are algebra-valued and the indices $A$ and $B$ range over the components of the

$G$-algebra. The matrix $D_{A B}=\operatorname{Tr}\left[T_{A} g T_{B} g^{-1}\right]$ relates the left and right invariant currents as $j_{a}^{A}=D_{B}^{A} \tilde{\mathrm{J}}_{a}^{B}$, with $T_{A}$ the generators of the group $G$. Based on its form, this action may be realized as an (integrable) deformation of the WZW model on level $k$ and this is what makes up the concept of the $\lambda$-deformation.

Getting back on our track, we choose the $\lambda$-model $\left[3.44\right.$ on $\mathrm{SU}(2) \cong \mathrm{S}^{3}[116]$, whose action is equivalent to bosonic string theory on the vacuum 


$$
\begin{aligned}
\mathrm{d} s_{\lambda}^{2} & =2 k\left(\frac{1+\lambda}{1-\lambda} \mathrm{d} z^{2}+\frac{1-\lambda^{2}}{\Delta} \sin ^{2} z \mathrm{~d} \Omega_{2}^{2}\right), \\
B_{2}^{\lambda} & =-2 k\left(z-\frac{(1-\lambda)^{2}}{\Delta} \cos z \sin z\right) \operatorname{vol} \Omega_{2}, \\
e^{-2 \Phi^{\lambda}} & =e^{-2 \Phi_{0}} \Delta,
\end{aligned}
$$

where $\Delta=1+\lambda^{2}-2 \lambda \cos 2 z$ and $\lambda \in[0,1]$. For $\lambda=0$ we obtain the original WZW model. The action we obtain for $\lambda \rightarrow 1$ is related to the non-Abelian T-dual of the WZW model in eq. (3.43), see [116 for a detailed explanation.

The SU(2) $\lambda$-model for $\lambda=3-2 \sqrt{2}$

Our intuition paid off. Comparing the $\mathrm{SU}(2) \lambda$-model in 3.45 with the special vacuum in (3.41), we notice that for $\lambda=3-2 \sqrt{2}$ those two coincide on the $\mathcal{M}^{3}$ part of the supergravity vacuum. In particular, for this value of $\lambda$, which implies $\Delta=4 \lambda\left(1+\sin ^{2} z\right)$, the $\lambda$-model 3.45 reads

$$
\begin{aligned}
\mathrm{d} s_{\lambda}^{2} & =2 \sqrt{2} k\left(\mathrm{~d} z^{2}+\frac{\sin ^{2} z}{1+\sin ^{2} z} \mathrm{~d} \Omega_{2}^{2}\right), \\
B_{2}^{\lambda} & =-2 k\left(z-\frac{\sin z \cos z}{1+\sin ^{2} z}\right) \operatorname{vol} \Omega_{2}, \\
e^{-2 \Phi^{\lambda}} & =e^{-2 \Phi_{0}^{\lambda}}(12-8 \sqrt{2})\left(1+\sin ^{2} z\right) .
\end{aligned}
$$

which is identical to the Neveu-Schwarz sector of the internal space $\mathcal{M}^{3}$ in 3.41, if we identify $\omega=\frac{\pi}{2 k}$ and choose a convenient dilaton field, $e^{-2 \Phi_{0}^{\lambda}}$. The holographic limit $\omega \rightarrow 0$, associated to long quivers, corresponds to $k \rightarrow \infty$, the semi-classical limit of the WZW model.

A word of caution here. $\mathrm{AdS}_{7} \times \mathrm{S}^{3}$ is not a supergravity solution, $\mathrm{AdS}_{7} \times \mathcal{M}^{3}$ as in 3.41 is. The $\lambda$-deformation was only used to connect (a part of) our vacuum to an integrable structure (the WZW on $\mathrm{S}^{3}$, in this case), not surf along different supergravity solutions.

Therefore, we have indeed spotted classical integrability for the special vacuum, following from the choice $\alpha(z)=A \sin (\omega z)$. It is for this choice of the function $\alpha(z)$ where the geometry becomes a direct product $\mathrm{AdS}_{7} \times \mathcal{M}^{3}$ and the Polyakov action factorizes into two 
separate $\sigma$-models (up to the Virasoro constraint), one for $\mathrm{AdS}_{7}$ and one for $\mathcal{M}^{3}$ coupled to a $B_{2}$ field. The first is bosonic-string dynamics in a symmetric space and, as also illustrated below, is integrable. The second, as was shown in this section, is an integrable $\lambda$-model. Thus, the only formal ingredient left to validate this particular story of classical integrability is a Lax connection and this is what we show next.

\subsection{The Lax connection}

The Lax connection is a connection on the cotangent bundle of the theory, whose flatness condition equals the dynamical equations of motion. This, together with the involution between all the analogous independent conserved quantities, reflects classical, Liouvillian integrability of the theory. In this section, we give some basic concepts and results, while a more thorough analysis and references are held in Appendix F.

\section{Lax connection on $\mathrm{AdS}_{7}$}

As stated repeatedly in this section, the $\sigma$-model on a symmetric space is classically integrable. In other words, the Polyakov action on an $\mathrm{AdS}_{n}$ target space, without a $B_{2}$-field, is integrable. In order to see this, we first introduce the PCM on a semisimple Lie group $G$,

$$
S_{P C M}=-\frac{\kappa^{2}}{2 \pi} \int d^{2} \sigma \operatorname{Tr}\left[\partial_{a} g \partial^{a} g^{-1}\right], \quad g \in G
$$

which exhibits a $G_{L} \times G_{R}$ global symmetry and can be written in terms of the Maurer-Cartan form $j_{a}$, an algebra-valued connection on the group manifold $G$,

$$
S_{P C M}=\frac{\kappa^{2}}{2 \pi} \int d^{2} \sigma \operatorname{Tr}\left[j_{a} j^{a}\right], \quad j_{a}=g^{-1} \partial_{a} g \in \mathfrak{g}
$$

where $\mathfrak{g}$ is the Lie algebra of $G$. This Maurer-Cartan form is by construction flat. The flatness condition together with the equations of motion for the action in eq.(3.48) read

$$
\begin{aligned}
\partial_{+} j_{-}+\partial_{-} j_{+} & =0, \\
\partial_{-} j_{+}-\partial_{+} j_{-}-\left[j_{+}, j_{-}\right] & =0 .
\end{aligned}
$$

Here we used lightcone coordinates on the string worldsheet. The above eqs. (3.49) combine to construct a Lax connection

$$
\mathcal{L}_{ \pm}=\frac{1}{1 \mp Z} j_{ \pm}
$$


where $Z \in \mathbb{C}$ is the spectral parameter, such that the flatness of the Lax connection

$$
d \mathcal{L}+\mathcal{L} \wedge \mathcal{L}=0
$$

is equivalent to the equations of motion (3.49) obtained from the action (3.48).

Now, while there is the isomorphism

$$
\mathrm{AdS}_{n+1} \cong \frac{\mathrm{SO}(2, n)}{\mathrm{SO}(1, n)}
$$

which implies the example of $\mathrm{AdS}_{3} \cong \mathrm{SO}(1,2) \cong \mathrm{SL}(2, \mathbb{R})$, our case at hand, $\mathrm{AdS}_{7}$, is not a group manifold $G$ but a symmetric coset $F=G / H$. That means that there is a $\mathbb{Z}_{2}$ automorphism of the algebra of $G$, under which the latter decomposes as $\mathfrak{g}=\mathfrak{f} \oplus \mathfrak{h}$. Thus, the right action of $H$ is realized as a gauge symmetry and, by introducing a $\mathfrak{h}$-valued gauge field $B_{a}$, the new gauge-invariant PCM action reads

$$
S_{P C M}=\frac{\kappa^{2}}{2 \pi} \int d^{2} \sigma \operatorname{Tr}\left[J_{a} J^{a}\right], \quad J_{a}=j_{a}-B_{a}
$$

where we have defined the projection $J_{a}=P_{\mathfrak{f}}\left(j_{a}\right)$. The resulting equations of motion are

$$
D_{a} J^{a}=0, \quad D_{a}=\partial_{a}+\left[B_{a}, \cdot\right]
$$

while the new flatness condition

$$
\partial_{a} B_{b}-\partial_{b} B_{a}+\left[B_{a}, B_{b}\right]+D_{a} J_{b}-D_{b} J_{a}+\left[J_{a}, J_{b}\right]=0
$$

uses the commutation relations $[\mathfrak{h}, \mathfrak{h}] \subset \mathfrak{h},[\mathfrak{h}, \mathfrak{f}] \subset \mathfrak{f}$ and $[\mathfrak{f}, \mathfrak{f}] \subset \mathfrak{h}$ to decompose into two separate projections on the algebras $\mathfrak{h}, \mathfrak{f}$ as

$$
\begin{aligned}
\partial_{a} B_{b}-\partial_{b} B_{a}+\left[B_{a}, B_{b}\right]+\left[J_{a}, J_{b}\right] & =0, \\
D_{a} J_{b}-D_{b} J_{a} & =0 .
\end{aligned}
$$

As before, the flatness condition (3.56) together with the equations of motion (3.54) combine into a Lax connection for the coset space, 


$$
\mathcal{L}_{ \pm}=B_{ \pm}+Z^{ \pm 1} J_{ \pm}
$$

whose flatness condition is equivalent the equations of motion of the PCM on the symmetric coset. This demonstrates that the string on a symmetric space, in the absence of a $B_{2}$ field, is classically integrable.

In Appendix F we introduce another natural but more geometric environment to realize the symmetric PCM and, hence, its Lax connection. We now move on to the $\mathcal{M}^{3}$ part of the vacuum.

\section{Lax connection on $\mathcal{M}^{3}$}

Since we proved that the $\mathcal{M}^{3}$ part of the vacuum is an (integrable) $\mathrm{SU}(2) \lambda$-model, for $\lambda=3-2 \sqrt{2}$, we may just write down its Lax connection, 116, as

$$
\mathcal{L}_{ \pm}=-\left(1+\frac{\sqrt{2}}{2}\right) \frac{A_{ \pm}}{1 \mp Z}
$$

where $Z$ is the spectral parameter and $A$ is an algebra-valued gauge field on $\mathrm{SU}(2)$ transforming as $A \rightarrow h^{-1} A h-h^{-1} \mathrm{~d} h$, with $h \in \mathrm{SU}(2)$. In our case, this gauge connection reads

$A_{ \pm}=\left(\begin{array}{c} \pm \sin \chi \sin \xi z_{ \pm} \pm \frac{\sin 2 z}{2\left(1+\sin ^{2} z\right)}\left(\cos \chi \sin \xi \chi_{ \pm}+\sin \chi \cos \xi \xi_{ \pm}\right)-\frac{\sin ^{2} z}{\sqrt{2}\left(1+\sin ^{2} z\right)}\left(2 \cos \xi \chi_{ \pm}-\sin 2 \chi \sin \xi \xi_{ \pm}\right) \\ \mp \sin \chi \cos \xi z_{ \pm} \pm \frac{\sin 2 z}{2\left(1+\sin ^{2} z\right)}\left(\cos \chi \cos \xi \chi_{ \pm}-\sin \chi \sin \xi \xi_{ \pm}\right)-\frac{\sin ^{2} z}{\sqrt{2}\left(1+\sin ^{2} z\right)}\left(2 \sin \xi \chi_{ \pm}+\sin 2 \chi \cos \xi \xi_{ \pm}\right) \\ \mp \cos \chi z_{ \pm}+\frac{\sin \chi}{2\left(1+\sin ^{2} z\right)}\left( \pm \sin 2 z \chi_{ \pm}+2 \sqrt{2} \sin ^{2} z \sin \chi \xi_{ \pm}\right)\end{array}\right)$

Here, for the variables, we use the notation $f_{+}=\partial_{+} f, f_{-}=\partial_{-} f$ and $f_{+-}=f_{-+}=\partial_{+} \partial_{-} f$, while we wrote $A_{ \pm}$in the adjoint representation of $S U(2)$. One may check that the flatness condition for this Lax connection is equivalent to the equations of motion for the Polyakov action on the internal space $\mathcal{M}^{3}$. Those dynamical equations are

$z_{+-}=\frac{1}{2 \sqrt{2}\left(\sin ^{2} z+1\right)^{2}}\left(\sqrt{2} \sin 2 z\left(\chi_{+} \chi_{-}+\sin ^{2} \chi \xi_{+} \xi_{-}\right)-\left(5+\sin ^{2} z\right) \sin ^{2} z \sin \chi\left(\chi_{+} \xi_{-}-\chi_{-} \xi_{+}\right)\right)$ 


$$
\begin{aligned}
& \chi_{+-}=\frac{1}{2} \sin 2 \chi \xi_{+} \xi_{-}+\frac{1}{2 \sqrt{2}}\left(1+\frac{4}{1+\sin ^{2} z}\right) \sin \chi\left(z_{+} \xi_{-}-z_{-} \xi_{+}\right)-\frac{\cot z}{1+\sin ^{2} z}\left(z_{+} \chi_{-}+z_{-} \chi_{+}\right), \\
& \xi_{+-}=-\frac{1}{2 \sqrt{2}}\left(1+\frac{4}{1+\sin ^{2} z}\right) \sin ^{-1} \chi\left(z_{+} \chi_{-}-z_{-} \chi_{+}\right)-\frac{\cot z}{1+\sin ^{2} z}\left(z_{+} \xi_{-}+z_{-} \xi_{+}\right)-\frac{\cos \chi}{\sin \chi}\left(\chi_{+} \xi_{-}+\chi_{-} \xi_{+}\right)
\end{aligned}
$$

and this concludes the formal construction of a Lax connection for our special vacuum, demonstrating classical integrability for bosonic string theory on it.

As a closing comment, we note that there are other examples in the literature of integrable supergravity vacua, whose geometries are direct products of integrable sub-spaces. Those examples are the Sfetsos-Thompson vacuum [75] in the Gaiotto-Maldacena class of supergravity solutions, the Lunin-Maldacena real $\beta$-deformations [27 and, of course, the special cases of [2] in Chapter 1; in all those cases the AdS warp factor is a constant. Similar observations have been made in [64].

\subsection{The dual field theory}

We now present an approach to the six-dimensional SCFT dual to the special, integrable vacuum in 3.41 . Since the derivative of the rank function, $R^{\prime}(z)=-\frac{\alpha^{\prime \prime \prime}(z)}{81 \pi^{2}}$, is not piecewise discontinuous and constant for this vacuum, a description in terms of a well-defined, six-dimensional quiver structure with gauge and flavor nodes, as those given in Section 2 , is not a suitable one. This is because the discontinuity of $R^{\prime}(z)$, the jumps in the slope of $R(z)$, is the feature that translates into ranks of gauge and flavor groups; this is absent for the continuous $R^{\prime}(z)$ of the special vacuum. Instead, we will define this particular SCFT by calculating some of its characterizing quantities.

First, it is illustrative to acquire the function $\alpha(z)=A \sin (\omega z)$ through a different path, other than the one of this section where the choice of $\alpha(z)$ followed from demanding a constant AdS warp factor. We begin from the defining equation for $\alpha(z)$, (3.5), that is

$$
\alpha^{\prime \prime \prime}=-162 \pi^{3} F_{0},
$$

which may also be regarded as an equation of motion. The solution of this equation, with $F_{0}$ being piece-wise constant (and possibly discontinuous), is a polynomial 


$$
\alpha(z)=a_{0}+a_{1} z+\frac{a_{2}}{2} z^{2}-\frac{162 \pi^{3} F_{0}}{6} z^{3},
$$

which is piece-wise continuous along the $P$ intervals to which the $z$-coordinate is divided. And, of course, every polynomial may be expanded in a Fourier series. In particular, demanding $F_{0}$ to be an even (periodic) function on that coordinate (with period $T=2 P$ ), equation 3.61) implies the Fourier expansion $\alpha^{\prime \prime \prime}(z)=\sum_{n} b_{n} \cos \left(\omega_{n} z\right)$. Integrating three times, we acquire

$$
\alpha(z)=\sum_{n=1}^{\infty} c_{n} \sin \left(\frac{n \pi}{N_{5}} z\right) .
$$

where we set all integration constants to zero, in order to satisfy the boundary conditions $\alpha(z=0)=\alpha\left(z_{f}\right)=0$. We also used that $Q_{N S 5}=N_{5}=z_{f}$, coming from (3.8). It is this infinite sum of harmonics that reproduces the piece-wise continuous function $\alpha(z)$, made out of cubic polynomials in each interval. In reverse, it may be the case that a particular polynomial $\alpha(z)$ may be well approximated by a particular harmonic and, hence, this is a way to realize $\alpha(z)=A \sin (\omega z)$ which defines the special, integrable vacuum. The physical content of such an harmonic, as discussed below the vacuum (3.41) and is evident from the form of $F_{0}$ in (3.61), is associated with D8-branes that are smeared along the $z$-dimension. This is in contrast with the case of sharply-localized D8-branes, which characterize the generic vacua of this supergravity family and correspond to a piece-wise constant $\alpha^{\prime \prime \prime}$, as with the examples in Section 2.1. A situation of this sort was also suggested in 61] and 43]. In [43], it was observed that a possible scaling under which the vacua of the form (3.3) are trustable representations of $\mathcal{N}=(1,0)$ SCFTs, involved taking the number of D8-branes to infinity and creating a continuous distribution; in that case, it was emphasized that anomaly cancellation still holds true.

Let us now outline the special, six-dimensional SCFT by exploring some basic quantities in its dual vacuum. We begin by considering the solution derived from $\alpha(z)=A \sin (\omega z)$ and choosing $\omega=\frac{n \pi}{N_{5}}$, which makes the $z$-coordinate range between $0 \leq z \leq \frac{N_{5}}{n}$. We work with $n=1$ only (the first harmonic) in what follows. Using again that $Q_{N S 5}=N_{5}=z_{f}$, we may employ equations (3.13) and (3.16) to calculate the number of D6 and D8-branes in this vacuum, 


$$
\begin{aligned}
& N_{D 6}=-\frac{1}{81 \pi^{2}} \int_{0}^{z_{f}=N_{5}} \alpha^{\prime \prime}(z) \mathrm{d} z=\frac{2 A \pi}{81 N_{5}}, \\
& N_{D 8}=\frac{1}{81 \pi^{2}}\left[\alpha^{\prime \prime \prime}(0)-\alpha^{\prime \prime \prime}\left(N_{5}\right)\right]=-\frac{2 A \pi}{81 N_{5}^{3}} .
\end{aligned}
$$

where we included a factor of $\pi$ in the first equation, coming from (3.13). (3.13) is a proposed formula, originally constructed by integration of the Page charge; it, now, has to include an appropriate factor of $\pi$ in order for the result to be a rational number. In absolute value, those expressions imply relations among the quantities,

$$
A=\frac{81}{2 \pi} N_{5} N_{D 6}, \quad A=\frac{81}{2 \pi} N_{5}^{3} N_{D 8} \quad \rightarrow \quad N_{D 6}=N_{D 8} N_{5}^{2},
$$

which defines the constant $A$ inside $\alpha(z)$. Subsequently, we may use 3.25 to find the holographic central charge for the special vacuum and its dual SCFT,

$$
c=-\frac{2^{8}}{3^{8} 16 G_{N}} \int_{0}^{z_{f}=N_{5}} \alpha(z) \alpha^{\prime \prime}(z) \mathrm{d} z=\frac{8}{3^{8} G_{N}} A^{2} \omega^{2} N_{5}=\frac{N_{D 6}^{2} N_{5}}{4 \pi^{2}},
$$

in terms of the number of D6 and NS5-branes, where we also used the convention $G_{N}=8 \pi^{6}$. In the same vein, we may use (3.18) to find the (regularized) entanglement entropy for this field theory,

$$
S_{E E}^{r e g}=\left(\frac{\mu_{1} \mu_{2}^{4}}{L^{4}}\right) 64 \pi^{4} N_{D 6}^{2} N_{5}
$$

which has the same scaling of $N_{5}$ and $N_{D 6}$ with the central charge. Of course, this common dependence on the matter content of the theory is as it should, since both quantities measure the degrees of freedom.

As already argued, $\alpha(z)=A \sin (\omega z)$ does not determine the ranks of gauge and flavor groups and a quiver-theory description is obscure for the SCFT dual to the special, integrable vacuum. Hence, the objects calculated in this subsection may serve as a description of the six-dimensional $\mathcal{N}=(1,0) \mathrm{SCFT}$, in the limit where the number of D-branes are large. 


\section{Conclusions}

In this chapter, we studied classical integrability on a class of type IIA vacua that preserve $\mathcal{N}=(0,1)$ supersymmetry, consist of a warped $\mathrm{AdS}_{7} \times \mathcal{M}^{3}$ geometry, with $\mathcal{M}^{3}$ isomorphic to $\mathrm{S}^{3}$, and are associated with D8-D6-NS5 Hanany-Witten brane set-ups, 36 43. The aim here was to spot any vacua in this supergravity family that may exhibit integrability and use holography to learn about their dual, six-dimensional $\mathcal{N}=(0,1)$ SCFTs.

Applying our methods of non-integrability on the dynamics of a particular bosonic string soliton, we illustrated that all vacua with a warped geometry are non-integrable, with the bright exception of a special vacuum with an unwarped $\mathrm{AdS}_{7} \times \mathcal{M}^{3}$ space, for which we proved the opposite to be true. In the latter case, that is when the geometry becomes a direct product $\mathrm{AdS}_{7} \times \mathcal{M}^{3}$, the Polyakov action factorizes into two separate $\sigma$-models (up to the Virasoro constraint), one for $\mathrm{AdS}_{7}$ and one for $\mathcal{M}^{3}$ coupled to a $B_{2}$ field. The first reflects bosonic-string dynamics in a symmetric space and is integrable, while the second proved to be an (integrable) $\mathrm{SU}(2) \lambda$-model, for a particular value of $\lambda$. The classical integrability of the special vacuum was formally concluded by the construction of a Lax connection, whose flatness condition reproduces the equations of motion of the dynamical system.

As a matter of fact, we noted that there are various examples in the literature of integrable supergravity vacua, in all of which cases the AdS warp factor is a constant and the geometries reduce to direct products of integrable sub-spaces. Those examples are the Sfetsos-Thompson vacuum $[75]$ in the Gaiotto-Maldacena class of supergravity solutions, the Lunin-Maldacena real $\beta$-deformations [27] and, of course, the special cases of [2] in Chapter 1, while similar observations were made in 64].

Nonetheless, while the six-dimensional field theories, dual to these $\mathrm{AdS}_{7}$ type IIA vacua, are described by quiver chains of gauge theories coupled through bifundamental matter, the special SCFT dual to the integrable vacuum cannot be represented by such a structure. This is because, in terms of string theory, this vacuum is flooded with D8-branes which make up a continuous distribution along the $z$-dimension. In terms of holography, this situation cannot determine the flavor and gauge groups for the dual field theory and, instead, this particular $\mathcal{N}=(1,0)$ SCFT was defined by some of its universal quantities, the central charge and entanglement entropy, which depend on the matter content in the field theory.

Finally, this chapter concludes the study of classical, Liouvillian integrability on string theory vacua. We mostly utilized our methods of non-integrability and, indeed, proved several classes of supergravity vacua as non-integrable, but, in the end, we also managed to spot a particular integrable $\mathrm{AdS}_{7}$ vacuum through the $\lambda$-deformations. Integrability itself is quite rare, considering the rich variety of supergravity vacua, and, certainly, relatively hard to prove, all of which facts render the tools of non-integrability reasonably appealing. As 
far as those tools are concerned, an important contribution comes from Chapter 1 where the proper use of Kovacic's theorem was illustrated, an analytic method that can handle parametrized systems of equations. In the context of string theory, whenever we have a parametrized supergravity family of solutions (where the parameters range over the various vacua in a family), this method informs us about possible, integrable solutions. Nonetheless, despite its rich structure in the physics of the string, integrability is, most notably, important for field theory. This stems from the fact that integrable field theories are solvable for any value of their coupling constant. In other words, the exploration of integrable structures in string theory is immensely useful in the context of holography, through which we can spot integrable CFTs, a project, if not impossible, otherwise quite demanding on its own right. 


\section{E Analytic non-integrability}

We begin by examining the NVE for $\varphi(\tau)$, following the concepts and notation of Chapter 1 and its Appendix A. That is, as in (3.39), we allow for small fluctuations $\varphi(\tau)=0+\epsilon f(\tau)$ in the $\varphi(\tau)$ equation of motion in 3.33 and acquire

$$
\begin{aligned}
& \ddot{f}(\tau)+\mathcal{B}(\tau) \dot{f}(\tau)+\mathcal{A}(\tau) f(\tau)=0 \\
& \mathcal{B}_{f}(\tau)=\left.\frac{f_{1}^{\prime}(z)}{f_{1}(z)} \frac{E}{4 \pi}\right|_{z_{s o l}}=\left.\frac{E}{8 \pi}\left(\frac{\alpha^{\prime}}{\alpha}-\frac{\alpha^{\prime \prime \prime}}{\alpha^{\prime \prime}}\right)\right|_{z_{s o l}} \\
& \mathcal{A}_{f}(\tau)=\mu^{2}
\end{aligned}
$$

Looking forward to apply Kovacic's theorem [45, we follow Chapter 1 and change variables as $y=e^{\frac{1}{2} \int \mathcal{B}} x$ in the above differential equation, deducing

$$
x^{\prime \prime}=\mathcal{V}_{x} x, \quad \mathcal{V}_{x}=\frac{1}{4}\left(2 \mathcal{B}_{y}^{\prime}+\mathcal{B}_{y}^{2}-4 \mathcal{A}_{y}\right)
$$

where $y$ is Liouvillian if and only if $x$ is Liouvillian and, thus, no generality is lost. In this new variable, the NVE for $\varphi$ is characterized by

$$
V_{f}(\tau)=\frac{-36 a_{3}^{4} E^{8} \mu^{2} \tau^{8}-384 a_{2} a_{3}^{3} E^{7} \pi \mu^{2} \tau^{7}+\ldots}{4\left(4 a_{2} \pi+3 a_{3} E \tau\right)^{2}\left(64 a_{0} \pi^{3}+16 a_{1} E \pi^{2} \tau+4 a_{2} E^{2} \pi \tau^{2}+a_{3} E^{3} \tau^{3}\right)^{2}}
$$

where we have replaced $z=z(\tau)=z_{\text {sol }}(\tau)$ inside $\alpha(z)$. Kovacic's automatic (software) algorithm fails to solve this as it is, which means that the system is non-integrable for a generic choice of its parameters. Hence, we have to employ the analytic method of the theorem, in order to decide whether there are any special choices for the parameters $a_{i}$ that lead to Liouvillian solutions.

Before even going into the pole structure of $\mathcal{V}_{f}$, we may just notice that both the numerator and denominator contain terms with $\tau^{8}$, which means that the order of $V_{f}(\tau)$ at infinity is zero, as $V_{f}(\tau) \sim-\mu^{2}$ when $\tau \rightarrow \infty$. Hence, following Kovacic's theorem in Appendix A, $V_{f}$ may only satisfy the first and second of Kovacic's criteria and not the third one, which justifies our choice to pick the $\varphi$-NVE (instead of the rest) as the simplest case to work on. Going into the pole structure of $\mathcal{V}_{f}$, except the obvious pole at $\tau=-4 a_{2} \pi / 3 a_{3} E$, includes taking the cubic polynomial to a depressed form, $\tau^{3}+p \tau+q$ and then follow Car- 
dano's formula for cubic equations. Finding those poles (which are given in terms of cubic roots), following the steps in Kovacic's analytic method and respecting the constraints on the parameters $a_{i}$ opposed by our supergravity construction (through and around condition (3.6) ), we deduce that no Liouvillian solutions may occur. In other words, there is not even a single choice for the parameters $a_{i}$ that leads to integrability. The procedure is quite long and the expressions not enlightening at all, but the quality of the process is identical to the calculations in Appendix C of Chapter 1 and, thus, we omit its details here.

\section{F Integrability on the Symmetric $\sigma$-model}

Classical Liouvillian integrability for a Hamiltonian dynamical system, or for a field theory, emerges over the existence of a flat Lax connection $\mathcal{L}$, that is

$$
d \mathcal{L}+\mathcal{L} \wedge \mathcal{L}=0
$$

on the cotangent bundle $\mathcal{T}^{*} \mathcal{M}$ (phase space), together with the involution of all the analogous independent conserved quantities. Generally, though, there is no particular prescription for finding such a connection and one has to rely on their inspiration to address the problem.

However, given a 2-dimensional scalar field theory in a homogeneous space for a connected semisimple Lie group $G$, the action can be reformulated in terms of its underlying group structure as

$$
S_{P C M} \equiv-\frac{\kappa^{2}}{\pi} \int \operatorname{Tr} j_{a} j^{a}
$$

where the Lie-algebra-valued current $j \in \mathfrak{g}(G)$,

$$
j_{ \pm} \equiv g^{-1} \partial_{ \pm} g=j_{ \pm}^{i} \mathfrak{t}_{i}, \quad g \in G, \quad \mathfrak{t}_{i} \in \mathfrak{g},
$$

is defined over the group element $g=e^{X^{i} t_{i}}$, that is all the point transformations on the scalar field worldsheet, on the group manifold. This one-form current is by construction flat and its flatness condition, together with the equations of motion,

$$
\begin{array}{r}
\partial_{+} j_{-}+\partial_{-} j_{+}=0, \\
\partial_{+} j_{-}-\partial_{-} j_{+}+\left[j_{+}, j_{-}\right]=0,
\end{array}
$$


may be combined in a parametrized Lax connection

$$
\mathcal{L}_{ \pm}=\frac{j_{ \pm}}{1 \mp Z}
$$

where $Z \in \mathbb{C}$ is the spectral parameter, whose flatness condition, eq. 3.72 , is equivalent to the equations of motion, eq. 3.75 . Then, one also defines the holonomy of $\mathcal{L}$ for constant time, i.e. the monodromy

$$
M(Z)=\mathcal{P} \exp \int \mathcal{L}
$$

which defines a parallel transport on the group manifold $\Sigma(G)$ and whose eigenvalues are conserved, which means that by expanding in $Z$ at infinity we can obtain an infinite set of conserved charges. This is known in the literature as the Principal Chiral Model (PCM), it exhibits a global $G_{L} \times G_{R}$ symmetry and it is obviously integrable.

Moreover, the $\sigma$-model (in the presence of a $B_{2}$ field) in a homogeneous space for a group $G$ can be represented by the WZW model as

$$
S_{W Z W, k}=\frac{k}{2 \pi} \int_{\partial \mathcal{B}} \operatorname{Tr} j_{a} j^{a}+\frac{k}{6 \pi} \int_{\mathcal{B}} \epsilon_{a b c} \operatorname{Tr} j^{a} j^{b} j^{c}, \quad j \in \mathfrak{g}(G)
$$

which exhibits an $G_{L, \text { cur }} \times G_{R, \text { cur }}$ current algebra symmetry, it is an exact CFT and thus integrable.

The situation becomes even more elegant in the case of the non-linear $\sigma$-model in a symmetric homogeneous space. Symmetric spaces are backgrounds with rich underlying group structure, which can be exploited in a natural way to make the integrability of the $\sigma$-model manifest. From the group theoretical point of view, a symmetric space is a coset space $G / H$, where the isometry $G$ is a connected Lie group and the subgroup $H \subset G$ is its isotropy group. Then the $\sigma$-model (without a B-field) can be recast as a PCM with currents projected on the coset algebra. The WZW model on a symmetric coset, on the other hand, does not correspond to the $\sigma$-model on that space (except in the case of a group manifold) and exhibits alternative interpretations.

In what follows we will illustrate the classical integrability of the string worldsheet on a symmetric space. To study this in more detail see [44], for a more general review of integrability in the context of string theory [117] and AdS/CFT correspondence 24]. 


\section{Integrability of AdS space}

The $\sigma$-model on AdS space is integrable. We know this as a fact, since, as we illustrated above, the $\sigma$-model is integrable on every symmetric homogeneous space. Of course, an uneasy mind shall always ask for an explicit Lax formulation given a specific background, something that proves to be quite challenging as we climb higher in dimensions of the target space. The difficulty rests in the fact that finding the gauged group element (matrix) of the coset space becomes an involved task in higher dimensions.

Nevertheless, if one desires to make this portrait more delicate, they shall preserve the rich underlying group structure of the PCM, adopting at the same time a more geometric point of view.

In particular, one can realize the element of a group $G$ abstractly as

$$
g \equiv \exp X^{i} \mathfrak{t}_{i}
$$

where $\mathfrak{t}_{i} \in \mathfrak{g}(G)$ and $X^{i}$ parametrize the adjoint space, which produces another formulation of the PCM action as

$$
S_{P C M}=-\frac{\kappa^{2}}{\pi} \int d^{2} \sigma \eta_{i j} e_{\mu}^{i}(X) e_{\nu}^{j}(X) \partial_{+} X^{\mu} \partial_{-} X^{\nu}
$$

where $\eta_{i j}=\left\langle\mathfrak{t}_{i}, \mathfrak{t}_{j}\right\rangle$ is the metric on the Lie algebra $\mathfrak{g}$, defined by $\left[\mathfrak{t}_{i}, \mathfrak{t}_{j}\right]=f_{i j}{ }^{k} \mathfrak{t}_{k}$, while the vielbein

$$
e_{\mu}^{i}=\frac{\partial X^{i}}{\partial X^{\mu}}
$$

represents the relationship between the adjoint and the target space $\mathrm{e}^{2}$

Therefore, in this context, the vielbeins $e^{i}{ }_{\mu}$ represent the components of the symmetry transformations of $G$ or, equivalently, the Killing vectors of the manifold at hand. Subsequently, the vielbein is realized as the Maurer-Cartan connection

$$
j_{ \pm}^{i} \equiv e_{ \pm}^{i}=e_{\mu}^{i} \partial_{ \pm} X^{\mu}
$$

where $j_{ \pm}=j_{ \pm}^{i} \mathfrak{t}_{i}$, and satisfies the structural flatness condition

\footnotetext{
${ }^{2} i$ runs in the adjoint space of $G$ while $\mu$ spans the target space dimensions. The vielbeins represent a relationship between different bases, i.e. they express an object in different frames. As such, this relationship can exist between any kind of spaces.
} 


$$
\partial_{\mu} e^{i}{ }_{\nu}-\partial_{\nu} e^{i}{ }_{\mu}+f^{i}{ }_{j k} e^{j}{ }_{\mu} e^{k}{ }_{\nu}=0 \text {. }
$$

As in the standard case, this flatness identity together with the equations of motion of the PCM

$$
\partial_{+}\left(e^{i}{ }_{\mu} \partial_{-} X^{\mu}\right)+\partial_{-}\left(e^{i}{ }_{\mu} \partial_{+} X^{\mu}\right)=0
$$

construct the Lax connection

$$
\mathcal{L}_{ \pm}=\frac{j_{ \pm}}{1 \mp Z}
$$

where $Z \in \mathbb{C}$ is the spectral parameter, and whose flatness condition

$$
\left[\partial_{+}+\mathcal{L}_{+}, \partial_{-}+\mathcal{L}_{-}\right]=0
$$

is equivalent to equations of motion.

Thus, we conclude that in order to specify a particular Lax connection for the $\sigma$-model on a symmetric space, one only needs the Killing vectors of the background manifold 3

The reader could argue that the Lax connection eq. 3.85 works only for the PCM on a group $G$, since it is not of the appropriate coset form, i.e. it doesn't project on separately the isotropy and coset algebras. However, this is not the case since, as we argued above, the Killing vectors are a special coset parametrization, constrained by the target space metric. In other words, as the Lax connection is defined up to a gauge transformation, one could gauge transform our Lax eq. 3.85) into a traditional coset Lax connection.

Next, finding the Killing vectors is, thankfully, a simple task for a symmetric space. This is because a symmetric space can always be realized as an embedding in a higher dimensional space, the former inheriting most of the isometries of the latter. A standard example is $S^{2}$ which inherits the $\mathrm{SO}(3)$ isometries from $\mathbb{R}^{3}$ (but not the translations).

$\mathrm{AdS}_{n}$ space is a hypersurface in $\mathbb{R}^{2, n-1}$ onto which only the Lorentz group is tangent. Therefore, the boosts and the rotations of $\mathbb{R}^{2, n-1}$,

$$
V_{i} \equiv V_{i}^{A} \partial_{Y^{A}}
$$

\footnotetext{
${ }^{3}$ One could be naively troubled about the fact that a symmetric space has less degrees of freedom than the number of its Killing vectors, e.g. $S^{2}$ has two d.o.f. and three Killing vectors. In reality, the Killing vectors - the space isometries - are constrained by the metric and encode the actual degrees of freedom.
} 
where $Y^{A}, A=0, \ldots, n$ are the embedding coordinates which build the hypersurface

$$
\eta_{A B} Y^{A} Y^{B}=-l^{2}
$$

with $\eta_{A B}=\operatorname{diag}(-1,1, \ldots,-1)$, are inherited into $\operatorname{AdS}_{n}$ as the Killing vectors

$$
\xi_{i} \equiv \xi_{i}^{\mu} \partial_{\mu}=g^{\mu \nu}\left(\frac{\partial Y^{A}}{\partial x^{\nu}} V_{A}\right)_{i} \partial_{\mu}
$$

where $x^{\mu}, \mu=0, \ldots, n-1$ are the $\mathrm{AdS}_{n}$ coordinates and $g_{\mu \nu}$ its metric, while $i$ runs in the vector space. By choosing one of the solutions to eq.(3.88), like the global embedding

$$
\begin{aligned}
Y^{0} & =l \cosh \rho \cos t, \\
Y^{j} & =l \sinh \rho \Omega^{j}, \\
Y^{n} & =l \cosh \rho \sin t,
\end{aligned}
$$

where $\Omega^{j}$ are the Euclidean coordinates for the unit sphere $\left(\Omega^{j} \Omega_{j}=1\right)$, one can find each one of the $n(n+1) / 2$ Killing vectors of $\mathrm{AdS}_{n}$.

It's worth emphasizing that the Killing vectors that are inherited into a symmetric space, through an embedding, are constrained by the metric tensor. This means that while their number (number of isometries) exceeds the dimension of the space, in reality they encode the actual degrees of freedom. In other words, the PCM metric

$$
G_{\mu \nu}=\eta_{i j} e_{\mu}^{i} e_{\nu}^{j} \partial_{+} X^{\mu} \partial_{-} X^{\nu}=\eta_{i j} \xi_{\mu}^{i} \xi^{j}{ }_{\nu} \partial_{+} X^{\mu} \partial_{-} X^{\nu}
$$

matches the target space metric (it has not redundant degrees of freedom). Thus, while in a matrix realization of the PCM we would, traditionally, have to gauge the isotropy group $H$ out of the isometry group $G$ to obtain the element of the coset $G / H$, the Killing vectors constitute a natural environment to describe a symmetric space.

Since we have identified the Killing vectors $\xi_{i}$ of the background space of the PCM with the vielbeins $e_{i}$ in eq. 3.80 , then one can explicitly check that the equations of motion of this action, eq. (3.84), are equivalent to the standard equations of motion of the $\sigma$-model in the same background, as they should. Therefore, the Killing vectors can be used to build up an explicit Lax connection through equations (3.82) and (3.85), as promised. 


\section{The $\mathrm{AdS}_{3}$ example}

While $\operatorname{AdS}_{n}$ can give frustrating results as we climb up the ladder of $n$, $\mathrm{AdS}_{3}$ constitutes a relatively compact example of the above methodology. The reader should not be worried about the special case of $\mathrm{AdS}_{3}$, it being a group manifold. As we argued above, our construction holds for every symmetric coset and, in fact, it was also tested for higher dimensions, successfully as it should.

Choosing a global $\mathrm{AdS}_{3}$ embedding in $\mathbb{R}^{2,2}$ as

$$
\begin{aligned}
& Y^{0}=\cosh \rho \cos t, \\
& Y^{1}=\sinh \rho \cos t \sin \phi, \\
& Y^{2}=\sinh \rho \cos t \cos \phi, \\
& Y^{3}=\cosh \rho \sin t,
\end{aligned}
$$

then the six corresponding Killing vectors are

$$
\begin{aligned}
& \xi_{1}=\partial_{t}, \\
& \xi_{2}=\partial_{\phi}, \\
& \xi_{3}=\tanh \rho \sin t \sin \phi \partial_{t}+\operatorname{coth} \rho \cos t \cos \phi \partial_{\phi}+\cos t \sin \phi \partial_{\rho}, \\
& \xi_{4}=\tanh \rho \sin t \cos \phi \partial_{t}-\operatorname{coth} \rho \cos t \sin \phi \partial_{\phi}+\cos t \cos \phi \partial_{\rho}, \\
& \xi_{5}=\tanh \rho \cos t \sin \phi \partial_{t}+\operatorname{coth} \rho \sin t \cos \phi \partial_{\phi}+\sin t \sin \phi \partial_{\rho}, \\
& \xi_{6}=\tanh \rho \cos t \cos \phi \partial_{t}-\operatorname{coth} \rho \sin t \sin \phi \partial_{\phi}+\sin t \cos \phi \partial_{\rho},
\end{aligned}
$$

where the curved indices of the components $\xi_{i}{ }^{\mu}$ can be lowered, as usual, with the global $\mathrm{AdS}_{3}$ metric $g_{\mu \nu}$. These Killing vectors $\xi_{i}$, as discussed before, are the vielbeins $e_{i}$ of the PCM action eq. 3.80 that construct the flat current eq. 3.82 , namely

$$
j_{ \pm}^{i}=\xi_{\mu}^{i} \partial_{ \pm} X^{\mu}
$$

from which the Lax connection in eq. 3.85 is built as

$$
\mathcal{L}_{ \pm}^{i}=\frac{j_{ \pm}^{i}}{1 \mp Z}
$$

The flatness eq. 3.86) of the PCM Lax connection results in two sets of equations, the first 
being the flatness eq. (3.83) of the Maurer-Cartan current, which is a structural fact as it can be easily checked by the reader. This is an identity to be expected, since this flatness equation can be realized as just the Cartan's first structure equation applied on Killing vectors.

The second set of equations are the equations of motion eq. (3.84) of the PCM, the necessary condition for an integrable model.

If one desires to further validate all the above, all they have to do is to secure the fact that the equations of motion of the PCM coincide with the equations of motion of the bosonic string, on $\mathrm{AdS}_{3}$.

For that purpose, we use the $\mathrm{AdS}_{3}$ Killing vectors, eq. 3.93), on the PCM equations of motion, eq. (3.84), that is

$$
\partial_{+}\left(\xi_{\mu}^{i} \partial_{-} X^{\mu}\right)+\partial_{-}\left(\xi_{\mu}^{i} \partial_{+} X^{\mu}\right)=0
$$

In particular, $\xi_{1}=e_{1}$ (which lifts to a boost in the $Y^{0}-Y^{3}$ plane of $\mathbb{R}^{2,2}$ ) gives

$$
\cosh \rho \partial_{+} \partial_{-} t=-\sinh \rho\left(\partial_{+} \rho \partial_{-} t+\partial_{+} t \partial_{-} \rho\right)
$$

which is the correct equation of motion for $t$, while $\xi_{2}=e_{2}$ (which lifts to a rotation in the $Y^{1}-Y^{2}$ plane of $\mathbb{R}^{2,2}$ ) gives

$$
\sinh \rho \partial_{+} \partial_{-} \phi=-\cosh \rho\left(\partial_{+} \rho \partial_{-} \phi+\partial_{+} \phi \partial_{-} \rho\right)
$$

which is the correct equation of motion for $\phi$. Last but not least, $\xi_{6}=e_{6}$ (which lifts to a rotation in the $Y^{2}-Y^{3}$ plane of $\mathbb{R}^{2,2}$ ), supplemented with the above equations for $t$ and $\phi$, gives

$$
\partial_{+} \partial_{-} \rho=\cosh \rho \sinh \rho\left(\partial_{+} \phi \partial_{-} \phi-\partial_{+} t \partial_{-} t\right)
$$

which, of course, is the correct equation of motion for $\rho$.

In accordance with what we have discussed so far, the fact that it took just three of the six Killing vectors of $\mathrm{AdS}_{3}$ to deduce the equations of motion is just another manifestation of the actual degrees freedom encoded in the Killing vectors. 




\section{Part II}

\section{Holographic duality}





\section{Part II preface}

In this second part, we employ holography and, in particular, the AdS/CFT duality, in order to explore the features of certain supersymmetric quantum field theories in two spacetime dimensions. This part is dedicated to the publication [4].

In Chapter 1 of the second part, the final chapter of this thesis, we study the duality between $\mathrm{AdS}_{3}$ massive Type IIA supergravity vacua and two-dimensional $\mathcal{N}=(0,4)$ quiver structures. More precisely, after categorizing all kinds of gravity solutions, we demystify the ones that seem to reflect anomalous gauge theories. In particular, we prove that there are bound states of D-branes on the boundary of the space which provide the dual quiver theory with exactly the correct amount of matter in order to cancel its gauge anomalies. Then we propose that the structure of the field theory should be complemented with additional bifundamental matter, which we argue it may only be $\mathcal{N}=(4,4)$ hypermultiplets. Finally, we construct a BPS string configuration and use the old and new supersymmetric matter to build its dual ultraviolet operator. During this holographic synthesis, we uncover some interesting features of the quiver superpotential and associate one of the proposed operators with the same classical mass of its dual BPS string. 



\section{Chapter 1}

\section{Holography for two-dimensional QFT}

\section{Introduction}

The AdS/CFT duality constitutes a primo realization of the holographic principle while it ties string theory to the most well-studied particle theories we possess. In other words, besides being a conceptual breakthrough on its own right, holography brings strong confidence that a complete quantum theory of gravity shines upon the physics of the superstring. Nonetheless, the power of this duality does not limit itself in supporting quantum gravity but also unravels the properties of certain supersymmetric quantum field theories that otherwise are yet out of our reach through the standard methods or techniques.

While over the years many type II supergravity solutions have made their appearance in the holographic arena, there is a certain kind that has recently been popping up more frequently and has become quite popular. These are supergravity backgrounds whose entirety of fields is defined by functions of the coordinates of the internal manifolds and are dual to supersymmetric quiver gauge theories. These are the kind of vacua that we considered in Chapter 1 and 3 . Studying those backgrounds ultimately boils down to understanding their defining functions. The dual physics of these vacua is generally described by SCFTs, which for $d<4$ are assumed to be strongly coupled IR fixed points that flow to better-understood ultraviolet quiver field theories through the renormalization group equations. The latter are defined on supersymmetric multiplets of fundamental fields, whose interactions are usually well-defined and provide an understandable particle theory.

SCFTs exist exclusively in $d<7$ dimensions 110 and there has been intensive work on all of their diversity, both field theoretically and holographically. In six dimensions, an infinite family of $\mathcal{N}=(0,1)$ theories has been discussed in [1, 36 43, 77, 97, 107, 109, as in Chapter 3. In five dimensions, solutions in a variety of supersymmetry were analyzed in 118 125. For $\mathcal{N}=2$ supersymmetry in four dimensions there has been a fruitful study in 62, 111, 126 130, while three dimensional $\mathcal{N}=4$ theories were discussed in 131 135.

The case of $\mathrm{AdS}_{3}$ supergravity solutions is somewhat unique. Three-dimensional gravity as well as the algebra of two-dimensional field theory make the study of $\mathrm{AdS}_{3}$ holography 
of particular interest and this is reflected on the rich literature regarding the subject, some representatives of which are 31, 136 150.

Another family of such $\mathrm{AdS}_{3}$ solutions was recently introduced in [31 34]. Those were the vacua considered in Chapter 1 and which we are about to consider in this chapter too, in more detail this time, focusing on different aspects of their structure. These massive IIA vacua are associated with D2-D4-D6-D8 Hanany-Witten brane set-ups 68 and were first build in 31. The D2 and D6-branes exist as fluxes and they are dual to gauge symmetries, while the D4 and D8-branes live explicitly in the background and provide dual flavor symmetries. In 33] a particular class of them that exhibits the local geometry $\mathrm{AdS}_{3} \times \mathrm{S}^{2} \times \mathrm{CY}_{2} \times \mathbb{R}$ was distinguished and was proposed to be dual to two-dimensional quiver quantum field theories with $\mathcal{N}=(0,4)$ supersymmetry. Some holographic aspects of these quivers were studied in 69, 151.

The defining functions of a supergravity solution render the form of the fields on the gravity side of the holographic duality, while they accordingly shape the exact structure of the dual quiver field theory. In order to validate the duality and study the whole range of its potential, one should explore the various properties of these functions and confirm that every single time they make perfect sense on their dual field-theoretical attribution. This makes up the starting point of this chapter, where we take the most unusual choice of such defining functions which seems to give an anomalous dual quantum field theory. By carefully focusing on the right regions of the supergravity background we discover D-branes that are realized as extra matter with new global symmetries in the dual quiver structure, providing exactly the flavors needed to cancel the apparent gauge anomalies. Due to strong Ramond-Ramond fluxes on the boundary of the space these D-branes come exclusively in bound states, forming polarizations that provide flavor symmetries in an idiosyncratic way.

Observing the quiver structure of the theories under consideration, we realize that there must be some linking multiplets missing. Such multiplets bind color D2 with flavor D4-branes and color D6 with flavor D8-branes, while it is shown that those may only be $\mathcal{N}=(4,4)$ hypermultiplets corresponding to suspended superstrings between D2 and D4-branes or D6 and D8-branes in the ancestral Hanany-Witten set-up.

The existence of this new matter complements the quiver structure, while it seems to be also vital in the construction of the dual operator for a particular BPS string state. To be precise, after picking a semiclassical string configuration connecting two stacks of D-branes in the background, we prove that this is a BPS state and propose a string of scalar fields as its dual UV operator. While two-dimensional scalars have mass dimension zero implying a vanishing conformal dimension for that operator, we conclude that the latter property is attained non-perturbatively. That is, we bring to the surface the superpotential of the UV quiver theory to find interactions between the scalars inside the operator, supporting the idea of a totally non-perturbative anomalous dimension at the IR of the RG flow. Next, we find 
that scalars inside the vector superfields should obtain a VEV through a Fayet-Iliopoulos term due to the $\mathrm{U}(1)$ theory inside each $\mathrm{U}(N)$ gauge group. Superpotential interactions between the vector and hypermultiplets then dictate that bifundamental matter acquires a mass, ultimately associating the dual UV operator with a classical mass equal to that of the BPS string. Since the operator mass is a sum of all the individual scalar field masses, this renders the operator very much alike to a classical bound state of particles dual to a bound string state between D-branes. Finally, we construct an alternative choice of a dual operator, made out of spinor scalars which ultimately synthesize a bosonic quantity, which also seems to be a good holographic fit for the dual BPS string state.

The plan of this chapter is as follows. In Section 2 we review the massive IIA supergravity backgrounds and quantum field theory first constructed in [31]. While the basics of these vacua are repeated from Chapter 1, we go beyond them and present new features, focusing on the field theory aspects of the duality. We also give a brief but complete summary of two-dimensional $\mathcal{N}=(0,4)$ quantum field theory that is useful in understanding gauge anomalies, R-current charges and superpotentials between multiplets, all basic ingredients for the self-containment of the present work. In Section 3 we study special solutions of vacua that naively give anomalous quiver theories and show how these are canceled by flavor symmetries produced by dielectric branes on the boundary of the space. In Section 4 we illustrate that new matter should be added in the structure of the field theory in the form of $\mathcal{N}=(4,4)$ hypermultiplets. Finally, in Section 5 we construct a BPS string soliton and propose a couple of dual operators, one of which both seems to be associated with the same classical mass of the dual soliton.

\section{$2 \quad \mathbf{A d S}_{3}$ massive IIA vacua vs $\mathcal{N}=(0,4)$ theory}

\subsection{The supergravity solutions}

In Chapter 1, a new family of $\mathrm{AdS}_{3}$ massive IIA supergravity solutions with $\mathcal{N}=(0,4)$ supersymmetry was introduced. A subclass of these solutions with local geometry $\mathrm{AdS}_{3} \times \mathrm{S}^{2} \times \mathrm{CY}_{2} \times \mathrm{I}_{\rho}$ was conjectured in $32-34$ to be dual to $\mathcal{N}=(0,4)$ quiver quantum field theories in two dimensions. These vacua have an NS NS sector, in string frame,

$$
\begin{aligned}
& \mathrm{d} s^{2}=\frac{u}{\sqrt{h_{4} h_{8}}}\left(\mathrm{~d} s_{\mathrm{AdS}_{3}}^{2}+\frac{h_{4} h_{8}}{4 h_{4} h_{8}+\left(u^{\prime}\right)^{2}} \mathrm{~d} s_{\mathrm{S}^{2}}^{2}\right)+\frac{\sqrt{h_{4} h_{8}}}{u} \mathrm{~d} \rho^{2}+\sqrt{\frac{h_{4}}{h_{8}}} \mathrm{~d} s_{\mathrm{CY}_{2}}^{2}, \\
& B_{2}=\frac{1}{2}\left(2 k \pi-\rho+\frac{u u^{\prime}}{4 h_{4} h_{8}+\left(u^{\prime}\right)^{2}}\right) \operatorname{vol}\left(\mathrm{S}^{2}\right), \quad \quad e^{-\phi}=\frac{h_{8}^{\frac{3}{4}}}{2 h_{4}^{\frac{1}{4}} \sqrt{u}} \sqrt{4 h_{4} h_{8}+\left(u^{\prime}\right)^{2}},
\end{aligned}
$$


where $u, h_{4}, h_{8}$ are functions of the coordinate $\rho$, defining this family of supergravity backgrounds. Note that we also allow for large gauge transformations $B_{2} \rightarrow B_{2}+\pi k \mathrm{vol}_{S^{2}}$, every time we cross a $\rho$-interval $[2 \pi k, 2 \pi(k+1)]$, for $k=0, \ldots, P$. The RR sector reads

$$
\begin{aligned}
& \hat{F}_{0}=h_{8}^{\prime}, \quad \hat{F}_{2}=-\frac{1}{2}\left(h_{8}-h_{8}^{\prime}\left(\rho-2 \alpha^{\prime} \pi k\right)\right) \operatorname{vol}\left(\mathrm{S}^{2}\right), \\
& \hat{F}_{4}=\left(\partial_{\rho}\left(\frac{u u^{\prime}}{2 h_{4}}\right)+2 h_{8}\right) \mathrm{d} \rho \wedge \operatorname{vol}\left(\mathrm{AdS}_{3}\right)-h_{4}^{\prime} \operatorname{vol}\left(\mathrm{CY}_{2}\right),
\end{aligned}
$$

where $\hat{F}=e^{-B_{2}} \wedge F$ is the Page flux. These functions are locally constrained as

$$
h_{4}^{\prime \prime}=h_{8}^{\prime \prime}=u^{\prime \prime}=0
$$

where the first two equations come from the Bianchi identities, while the last comes from supersymmetry. This results in piecewise linear functions

$$
\begin{gathered}
h_{4}(\rho)=\left\{\begin{array}{cc}
\alpha_{0}+\frac{\beta_{0}}{2 \pi} \rho & 0 \leq \rho \leq 2 \pi \\
\alpha_{k}+\frac{\beta_{k}}{2 \pi}(\rho-2 \pi k) & 2 \pi k \leq \rho \leq 2 \pi(k+1) \\
\alpha_{P}+\frac{\beta_{P}}{2 \pi}(\rho-2 \pi P) & 2 \pi P \leq \rho \leq 2 \pi(P+1)
\end{array} \quad k=1, \ldots, P-1,\right. \\
h_{8}(\rho)=\left\{\begin{array}{cc}
\mu_{0}+\frac{\nu_{0}}{2 \pi} \rho & 0 \leq \rho \leq 2 \pi \\
\mu_{k}+\frac{\nu_{k}}{2 \pi}(\rho-2 \pi k) & 2 \pi k \leq \rho \leq 2 \pi(k+1) \\
\mu_{P}+\frac{\nu_{P}}{2 \pi}(\rho-2 \pi P) & 2 \pi P \leq \rho \leq 2 \pi(P+1)
\end{array}\right.
\end{gathered}
$$

while $u=a+b \rho$ globally, for supersymmetry to be preserved. Note that $P, \alpha_{k}, \mu_{k}$ have to be large for the supergravity limit to be trusted, while continuity of these equations along $\rho$ implies $\mu_{k}=\sum_{i}^{k-1} \nu_{i}$ and $\alpha_{k}=\sum_{i}^{k-1} \beta_{i}$.

Nonetheless, the defining functions have to be chosen with some care for the space to properly close on the $\rho$-dimension. Considering a linear $u$ function, both $h_{4}, h_{8}$ need to be zero at the $\rho=0$ endpoint whereas at $\rho=2 \pi(P+1) \equiv \rho_{f}$ only one of them needs to vanish. For a constant $u$ function, on the other hand, just one of them has to vanish at any endpoint. The study in 32,33 focused exclusively on solutions where both of these defining functions vanish at the endpoints, i.e. for $\alpha_{0}=\mu_{0}=a=0$ and $\nu_{P}=-\mu_{P}, \beta_{P}=-\alpha_{P}$ in the above definitions (1.4) and 1.5), a particular choice being represented by Figure 1.1. In Section 3 of the present chapter, we investigate all other possible cases, where $h_{4}$ and $h_{8}$ generically do not vanish at the endpoints of the $\rho$-coordinate. 


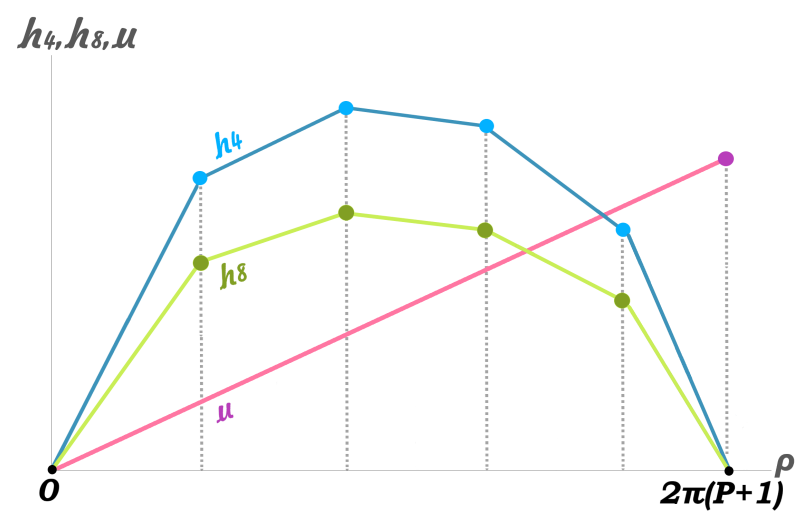

Figure 1.1: An example of piecewise linear functions $h_{4}, h_{8}$ and of $u$, defining a particular supergravity background. Here, both $h_{4}$ and $h_{8}$ vanish at the endpoints of the $\rho$-dimension.

This particular choice of backgrounds - where $h_{4}$ and $h_{8}$ are both zero at the endpoints of the $\rho$-dimension - start in a smooth fashion on this coordinate as the non-Abelian T-duals of $\mathrm{AdS}_{3} \times \mathrm{S}^{3} \times \mathrm{CY}_{2}$ 31. Near the endpoint $\rho=2 \pi(P+1)-x$ with $x \rightarrow 0$, on the other hand, the space becomes

$$
\mathrm{d} s^{2} \sim \frac{s_{1}}{x} \mathrm{~d} s_{\mathrm{AdS}_{3}}^{2}+s_{3} \mathrm{~d} s_{\mathrm{CY}_{2}}^{2}+\frac{x}{s_{1}}\left(\mathrm{~d} x^{2}+s_{1} s_{2} \mathrm{~d} s_{\mathrm{S}^{2}}^{2}\right), \quad e^{-4 \phi}=s_{4} x^{2},
$$

where $s_{i}$ are constants. According to the extremal $p$-brane solutions, classified in Appendix $\mathrm{G}$, this space is a superposition of $\mathrm{O} 2 / \mathrm{O} 6$ planes, where the $\mathrm{O} 2$ are smeared over O6.

In order to gain a better grip on the parameters of the system, let us consider the RR charges on the intervals $[2 \pi k, 2 \pi(k+1)]$. For $\alpha^{\prime}=g_{s}=1$, a $\mathrm{D} p$-brane is charged under $Q_{D p}=(2 \pi)^{p-7} \int_{\Sigma_{8-p}} \hat{F}_{8-p}$, thus in our set-up they read

$$
\begin{array}{ll}
Q_{D 2}=\frac{1}{32 \pi^{5}} \int_{\mathrm{CY}_{2} \times \mathrm{S}^{2}} \hat{F}_{6}=h_{4}-h_{4}^{\prime}(\rho-2 \pi k)=\alpha_{k}, & Q_{D 4}=\frac{1}{8 \pi^{3}} \int_{\mathrm{CY}_{2}} \hat{F}_{4}=\beta_{k}, \\
Q_{D 6}=\frac{1}{2 \pi} \int_{\mathrm{S}^{2}} \hat{F}_{2}=h_{8}-h_{8}^{\prime}(\rho-2 \pi k)=\mu_{k}, & Q_{D 8}=2 \pi F_{0}=2 \pi h_{8}^{\prime}=\nu_{k},
\end{array}
$$

Also, $Q_{N S}=\frac{1}{4 \pi^{2}} \int_{\rho \times S^{2}} H_{3}=1$, while we used that $\operatorname{vol}\left(\mathrm{CY}_{2}\right)=16 \pi^{4}$. These results imply that $\alpha_{k}, \beta_{k}, \mu_{k}, \nu_{k}$ are integers. A study of the Bianchi identities in the next section reveals that no explicit D2 and D6 branes are present in the geometry, just their fluxe: 1 . This associates their amount, $\alpha_{k}$ and $\mu_{k}$ respectively, with the ranks of the (color) gauge groups in the

\footnotetext{
${ }^{1}$ This is true when the worldvolume gauge field on the D8, D4 branes is absent. When it is on, as we are about to see, there is D6 and D2 flavor charge induced on the D8's and D4's.
} 


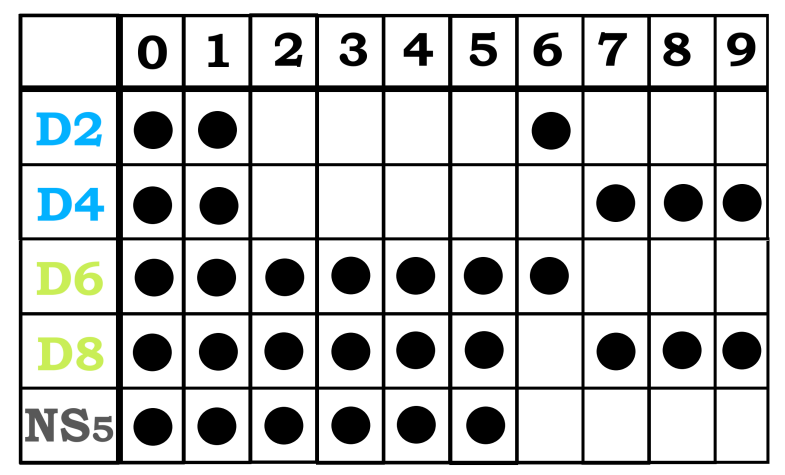

Table 1.1: $\frac{1}{8}$-BPS brane set-up, generator of our supergravity backgrounds. The dimensions $\left(x_{0}, x_{1}\right)$ are where the $2 \mathrm{~d}$ CFT lives. The dimensions $\left(x_{2}, \ldots, x_{5}\right)$ span the $\mathrm{CY}_{2}$, on which the D6 and the D8-branes are wrapped. The coordinate $x_{6}$ is associated with $\rho$. Finally $\left(x_{7}, x_{8}, x_{9}\right)$ are the transverse directions realizing an $\mathrm{SO}(3)$-symmetry associated with the isometries of $\mathrm{S}^{2}$.

dual field theory. On the other hand, as restated, D8 and D4 branes do exist in the geometry and modify the Bianchi identities by a delta function. Thus, $\beta_{k}$ and $\nu_{k}$ are associated with the ranks of the (flavor) global symmetries of the dual field theory.

\subsection{Bianchi identities}

The above story is conjectured 32,34 to be generated by a certain Hanany-Witten brane set-up 68. However, in this case the D-branes are not distributed across flat space as usual but along flat dimensions and a $\mathrm{CY}_{2}$ manifold instead, as indicated by Table 1.1 .

The family of supergravity backgrounds (1.1) comes to be as the near-horizon limit of this brane set-up, given always a large portion of each of the D-branes. Nevertheless, not all D-branes are explicitly present in the near-horizon limit of a Hanany-Witten set-up; some are there while others exist only as RR fluxes. This distinction is immensely important to Section 3 and, thus, to clarify the situation we turn our attention to the Bianchi identities.

We begin by noticing that $\mathrm{d} F_{0}=h_{8}^{\prime \prime} \mathrm{d} \rho$ and $\mathrm{d} \hat{F}_{4}=h_{4}^{\prime \prime} \mathrm{d} \rho \wedge \operatorname{vol}\left(\mathrm{CY}_{2}\right)$ where, according to the Bianchi identites encoded in (1.3), $h_{4}^{\prime \prime}=h_{8}^{\prime \prime}=0$ at a generic point along $\rho$. However, $h_{4}$ and $h_{8}$ are piecewise functions, given by 1.4 and (1.5), which means that at the points where their slope changes we get

$$
h_{4}^{\prime \prime}=\sum_{k=1}^{P}\left(\frac{\beta_{k-1}-\beta_{k}}{2 \pi}\right) \delta(\rho-2 k \pi), \quad h_{8}^{\prime \prime}=\sum_{k=1}^{P}\left(\frac{\nu_{k-1}-\nu_{k}}{2 \pi}\right) \delta(\rho-2 k \pi) .
$$


These give the source equations

$$
\begin{aligned}
& \mathrm{d} F_{0}=h_{8}^{\prime \prime} \mathrm{d} \rho, \quad \mathrm{d} \hat{F}_{6}=\mathrm{d} \hat{f}_{6}=\frac{1}{2} h_{4}^{\prime \prime}(\rho-2 k \pi) \mathrm{d} \rho \wedge \operatorname{vol}\left(\mathrm{S}^{2}\right) \wedge \operatorname{vol}\left(\mathrm{CY}_{2}\right), \\
& \mathrm{d} \hat{F}_{4}=\mathrm{d} \hat{f}_{4}=h_{4}^{\prime \prime} \mathrm{d} \rho \wedge \operatorname{vol}\left(\mathrm{CY}_{2}\right), \quad \mathrm{d} \hat{F}_{2}=\mathrm{d} \hat{f}_{2}=\frac{1}{2} h_{8}^{\prime \prime}(\rho-2 k \pi) \mathrm{d} \rho \wedge \operatorname{vol}\left(\mathrm{S}^{2}\right),
\end{aligned}
$$

indicating that there are localized D4 and/or D8 branes at points $\rho=2 k \pi$, whenever the slope between the intervals $[k-1, k]$ changes. In fact, the D4-branes are smeared over $\mathrm{CY}_{2}$, while note that $f_{p}$ represents the magnetic part of a RR flux $F_{p}$. We also use that $x \delta(x)=0$, which yields that there are no sources present for the D6 and D2-branes. This is because of the large gauge transformations of the Kalb-Ramond field.

The above source equations suggest that the D2 and D6-branes play the role of color branes, while the D4 and D8-branes that of flavor branes. Since gauge transformations vanish at infinity, it is the gauge fields fluctuating on the D4 or D8-branes in the bulk that are realized as global (flavor) symmetries in the dual field theory. Ultimately, the essential feature of the Bianchi identities which becomes crucial in the forthcoming analysis is that the derivatives of $h_{4}$ and $h_{8}$ source D4 and D8-branes, respectively.

In the above source equations, however, we have not considered the gauge fields living on the D4 and D8 branes. Switching on a gauge field $\tilde{f}_{2}$ on both kinds of D-branes, we form the gauge invariant field strength $\mathcal{F}_{2}=B_{2}+\lambda \tilde{f}_{2}$, where $\lambda=2 \pi l_{s}^{2}$, and the Bianchi identities now become

$$
\begin{aligned}
& \mathrm{d} \hat{f}_{2}=\lambda \tilde{f}_{2} \wedge \mathrm{d} F_{0}, \\
& \mathrm{~d} \hat{f}_{4}=h_{4}^{\prime \prime} \mathrm{d} \rho \wedge \operatorname{vol}\left(\mathrm{CY}_{2}\right)+\frac{\lambda^{2}}{2} \tilde{f}_{2} \wedge \tilde{f}_{2} \wedge \mathrm{d} F_{0}, \\
& \mathrm{~d} \hat{f}_{6}=\lambda \tilde{f}_{2} \wedge\left(h_{4}^{\prime \prime} \mathrm{d} \rho \wedge \operatorname{vol}\left(\mathrm{CY}_{2}\right)\right)+\frac{\lambda^{3}}{3 !} \tilde{f}_{2} \wedge \tilde{f}_{2} \wedge \tilde{f}_{2} \wedge \mathrm{d} F_{0}
\end{aligned}
$$

In regard to the gauge field dynamics, it being of order $l_{s}^{2}$, one may neglect it and keep only the zeroth order contribution, that is the Bianchi identities 1.9 that give only D8 and D4-branes; this is what was assumed in [32]. In Section 3 of the present chapter, however, we deal with cases where the gauge field does become important and completely redefines the supergravity picture on the boundaries of the space. 


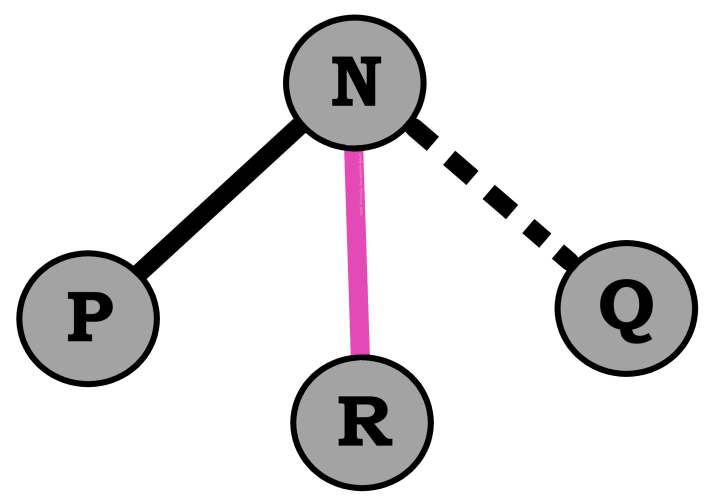

Figure 1.2: The building block of our quiver field theories. The solid black line represents a $\mathcal{N}=(4,4)$ hypermultiplet, the maroon line a $\mathcal{N}=(0,4)$ hypermultiplet and the dashed line represents a $\mathcal{N}=(0,2)$ Fermi multiplet. Inside the node representing an $\mathrm{SU}(N)$ gauge theory lives a $\mathcal{N}=(4,4)$ vector multiplet. The groups $\mathrm{SU}(P), \mathrm{SU}(Q)$ and $\mathrm{SU}(R)$ can be gauge or global symmetries.

\section{$2.3 \mathcal{N}=(0,4)$ SCFT}

The conjecture of [33] is that the above family of supergravity vacua is dual to a set of twodimensional SCFTs with $\mathcal{N}=(0,4)$ supersymmetry. These SCFTs are considered to be the low-energy fixed points on the RG flows of well-defined quantum field theories. Here, we just introduce the basic idea on those better-understood UV particle theories, ultimately aiming to cancel gauge anomalies that shall arise and also to unravel some interesting properties of the quiver superpotential. The $\mathcal{N}=(0,4)$ superfields are summarized in Appendix $\mathrm{H}$, at the end of this chapter, while a more thorough analysis on this kind of supersymmetry is held in Appendix 1, at the end of the thesis.

\section{Gauge anomalies}

The quiver gauge theory of 33 may be outlined by its fundamental building block of superfields, given by Figure 1.2. The field content and action of those multiplets is given in Appendix H.1 and, besides giving basic insight on the quiver structure, it is used in Section 5 to build an operator and challenge its interacting properties.

Each $\mathrm{SU}(N)$ gauge theory living on $N$ D2 or D6 color branes is represented by a gauge node that yields a $\mathcal{N}=(4,4)$ vector multiplet. In $\mathcal{N}=(0,2)$ language, each gauge node includes a vector, a Fermi and two twisted chiral multiplets in the adjoint representation of $\mathrm{SU}(N)$. A gauge node connects with other (gauge or flavor) nodes which in turn represent theories of (gauge or global) symmetry groups $\mathrm{SU}(P), \mathrm{SU}(R)$ and $\mathrm{SU}(Q)$, providing altogether a quiver network that reflects superstrings suspended between branes.

In the notation of Figure 1.2, the $\mathrm{SU}(N)$ gauge node connects to the $\mathrm{SU}(P)$ (gauge or flavor) node through a $\mathcal{N}=(4,4)$ hypermultiplet. In $\mathcal{N}=(0,2)$ language, each such 
hypermultiplet includes two Fermi and two chiral multiplets. Since there are $N P$ kinds of strings between the $\mathrm{SU}(N)$ and the $\mathrm{SU}(P)$ brane stacks, we realize $2 N P$ of each of these Fermi and chiral multiplets. The $\mathrm{SU}(N)$ gauge node also connects to a $\mathrm{SU}(R)$ node, through a $\mathcal{N}=(0,4)$ hypermultiplet. That is, through two $\mathcal{N}=(0,2)$ chiral multiplets. Since there are $N R$ kinds of strings between the $\mathrm{SU}(N)$ and the $\mathrm{SU}(R)$ brane stacks, we realize $2 N R$ chiral multiplets connecting the two nodes. In the same manner, the $\mathrm{SU}(N)$ gauge node connects to a $\mathrm{SU}(Q)$ node, through $N Q \mathcal{N}=(0,2)$ Fermi multiplets. All that being said, we may consider the superfield content of Appendix H.1 to find the overall anomaly of the gauge group $\mathrm{SU}(N)$ and impose that it cancels.

In two dimensions, quantum anomalies are one-loop-exact products of two point current correlations. The calculation is quite simple 152,153 and, given a non-Abelian symmetry acting on $\psi_{+i}$ right-moving and $\lambda_{-a}$ left-moving fermions with charges $Q_{R i}$ and $Q_{L a}$ respectively, it gives

$$
<J_{\mu}^{A} J_{\nu}^{B}>\sim \operatorname{Tr}\left[\gamma_{3} J_{\mu}^{A} J_{\nu}^{B}\right]=\operatorname{Tr}\left[T^{A} T^{B}\right]\left(\sum_{i} Q_{R i}^{2}-\sum_{a} Q_{L a}^{2}\right) \delta_{\mu \nu}
$$

where $T^{A}$ are the non-Abelian generators. For $\mathrm{SU}(N)$, the algebra has a metric

$$
\operatorname{Tr}\left[T^{A} T^{B}\right]=N \delta^{A B} \quad \text { or } \quad \operatorname{Tr}\left[T^{A} T^{B}\right]=\frac{1}{2} \delta^{A B}
$$

depending on the generators being in the adjoint or in the (anti-) fundamental representation, respectively. Obviously, no mixing between non-Abelian currents takes place.

Since gauge anomalies need to be always canceled for a consistent quantum field theory, chiral theories like ours require us to carefully study the anomaly contribution of each multiplet. Considering the field content previously presented, the $\mathrm{SU}(N)$ anomaly coming from the $\mathcal{N}=(0,2)$ superfields comes as follows:

- Vector superfield: they are in the adjoint representation of the gauge group $\mathrm{SU}(N)$ and thus they contribute with a factor of $-N$.

- Chiral superfield: if they are in the adjoint representation of the gauge group $\mathrm{SU}(N)$ they contribute with a factor of $N$. If they are in the (anti-) fundamental representation they contribute with a factor of $\frac{1}{2}$.

- Fermi superfield: if they are in the adjoint representation of the gauge group $\mathrm{SU}(N)$ they contribute with a factor of $-N$. If they are in the (anti-) fundamental representation they contribute with a factor of $\frac{1}{2}$. 
Having established all the gauge anomaly contributions, the next step is to use them on our kind of quantum field theories and see if there are any restrictions coming off the anomaly cancellation condition. This is a simple task since the proposed holographic duality describes a particular building block of supersymmetric multiplets that supports our quiver field theories. That is the one on Figure 1.2 .

With the quiver building block in mind, Figure 1.2 , we may calculate the overall anomaly of the gauge group $\mathrm{SU}(N)$ and impose that it cancels. Of course, the same job is to be done for each gauge group in a quiver field theory. For $\mathrm{SU}(N)$, as in Figure 1.2, the contributions come from the multiplets that couple to its gauge current, that is:

- $\mathcal{N}=(4,4)$ vector multiplet: the adjoint fields contribute as $2 N-N-N=0$. This is as it must since this is a vectorial multiplet, with equal amount of right and left-moving fermions.

- $\mathcal{N}=(4,4)$ hypermultiplet: the bifundamental fields connecting to $\mathrm{SU}(P)$ contribute as $2 N P\left(\frac{1}{2}-\frac{1}{2}\right)=0$. Again, this is expected since this hypermultiplet is too vectorial.

- $\mathcal{N}=(0,4)$ hypermultiplet: this connects to $\mathrm{SU}(R)$ and contributes as $2 N R \frac{1}{2}=N R$

- $\mathcal{N}=(0,2)$ Fermi multiplet: it connects to $\mathrm{SU}(Q)$ and contributes as $N Q\left(-\frac{1}{2}\right)$.

Requiring the gauge anomaly cancellation, we reach the condition

$$
2 R=Q
$$

which analogously must hold for each gauge group in a consistent quiver gauge theory.

If all the above is to hold, then the anomaly cancellation condition must agree with the dual situation on the supergravity side of the story. That is, since anomaly cancellation requires certain relationships between the ranks of the gauge and global symmetry groups, the amounts of branes (represented field theoretically by these ranks) in the supergravity side should be in total agreement with (1.13). This is indeed the case. Choosing an arbitrary supergravity solution where both $h_{4}$ and $h_{8}$ vanish at the end points of the $\rho$-dimension as

$h_{4}(\rho)=\left\{\begin{array}{cc}\frac{\beta_{0}}{2 \pi} \rho & 0 \leq \rho 2 \pi \\ \left(\beta_{0}+\beta_{1}+\ldots+\beta_{k-1}\right)+\frac{\beta_{k}}{2 \pi}(\rho-2 \pi k) & 2 \pi k \leq \rho \leq 2 \pi(k+1) \\ \alpha_{P}-\frac{\alpha_{P}}{2 \pi}(\rho-2 \pi P) & 2 \pi P \leq \rho \leq 2 \pi(P+1)\end{array} \quad k=1, \ldots, P-1\right.$, 


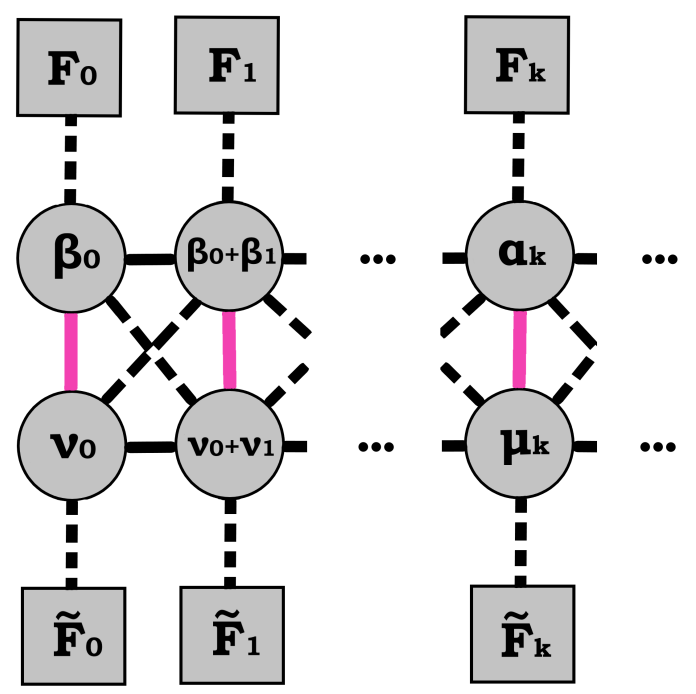

Figure 1.3: A standard example of a quiver field theory, dual to the proposed family of massive type IIA $\mathrm{AdS}_{3}$ supergravity solutions. This particular quiver theory reflects the solution defined by the functions $(1.14)$ and $(1.15)$. Circle nodes indicate gauge groups while square ones indicate global (flavor) symmetries. This figure is schematic in the sense that flavor nodes are actually far apart in the supergravity limit.

$h_{8}(\rho)=\left\{\begin{array}{cc}\frac{\nu_{0}}{2 \pi} \rho & 0 \leq \rho \leq 2 \pi \\ \left(\nu_{0}+\nu_{1}+\ldots+\nu_{k-1}\right)+\frac{\nu_{k}}{2 \pi}(\rho-2 \pi k) & 2 \pi k \leq \rho \leq 2 \pi(k+1) \\ \mu_{P}-\frac{\mu_{P}}{2 \pi}(\rho-2 \pi P) & 2 \pi P \leq \rho \leq 2 \pi(P+1)\end{array} \quad k=1, \ldots, P-1\right.$,

and $u=\frac{b_{0}}{2 \pi} \rho$, we make use of the Page charges 1.7 to decode these functions into portions of D-branes that give a dual quiver theory as in Figure 1.3 .

Applying the anomaly cancellation condition 1.13 on the gauge currents of the first gauge nodes of the above quiver chain we find

$$
\begin{array}{ll}
F_{0}+\nu_{0}+\nu_{1}=2 \nu_{0} & \Rightarrow \quad F_{0}=\nu_{0}-\nu_{1} \\
\tilde{F}_{0}+\beta_{0}+\beta_{1}=2 \beta_{0} & \Rightarrow \quad \tilde{F}_{0}=\beta_{0}-\beta_{1}
\end{array}
$$

which are precisely the results that we get from the Bianchi identities 1.8 for the portions of the D4 and D8-branes, validating further the proposed duality. 


\section{U(1) R-current anomaly}

Non critical for the consistency of the gauge theory but as much instructive is the anomaly produced by the R-symmetry current. Focusing on the $\mathrm{SU}(N)$ gauge theory of our building block and considering the $\mathrm{U}(1)_{R}$ R-charges that are given in Appendix H.2, we consider the anomaly contributions through $\operatorname{Tr}\left[\gamma_{3} Q_{i}^{2}\right]$ on each multiplet:

- For the fields in the adjoint representation of $\mathrm{SU}(N)$, the only contribution comes from the fermions inside the vector and Fermi multiplets. This amounts to a contribution of $-2\left(N^{2}-1\right)$. This coincides with (minus) twice the number of $\mathcal{N}=(0,4)$ vector multiples in $\mathrm{SU}(N)$.

- The contribution coming from the bifundamentals joining $\mathrm{SU}(N)$ with $\mathrm{SU}(P)$ is $2 N P$, due to both of the right-handed fermions inside each hypermultiplet. This is the number of $\mathcal{N}=(0,4)$ hypermultiplets in that link.

- The contribution coming from the fields running inside the maroon line, joining $\mathrm{SU}(N)$ with $\mathrm{SU}(R)$, is accordingly $2 N R$, once again counting the number of $\mathcal{N}=(0,4)$ hypermultiplets running on that connection.

- Finally, the fields running over the dashed line do not contribute as the left-handed fermion is uncharged under R-symmetry.

Summarizing, we find that the total R-anomaly reads

$$
\operatorname{Tr}\left[\gamma_{r} Q_{i}^{2}\right] \sim 2\left(n_{\text {hyp }}-n_{v e c}\right)
$$

which is proportional to the difference between the hypermultiplets and the vector superfields of the building block. As derived in 32,154 this anomaly is linked to the central charge of the theory

$$
c=6\left(n_{\text {hyp }}-n_{v e c}\right)
$$

which will be useful to us in Section 4, where we want to add matter in the theory while leaving this charge intact.

\section{Quiver superpotential}

As promised, we now realize a superpotential on our quiver theory by focusing on its building block given by Figure 1.2. In particular, we just take one simple connection of it, that is the 
link between a hypermultiplet and a vector superfield. All other links on the quiver structure can be deduced as generalizations of this connection. In fact, a particular two-dimensional superpotential was developed in 156 that serves exactly our case; we briefly reproduce this here, in order to extract the field interactions which furnish a certain operator in Section 5 with special features.

Through $\mathcal{N}=(0,2)$ supersymmetric eyes, a $\mathcal{N}=(4,4)$ vector superfield breaks into a vector multiplet $\mathcal{V}$, a Fermi multiplet $\Theta$ and two (twisted) chiral multiplets $\Sigma, \tilde{\Sigma}$. On the other hand, a $\mathcal{N}=(4,4)$ hypermultiplet breaks into two chiral multiplets $\Phi, \tilde{\Phi}$ and two Fermi multiplets $\Gamma, \tilde{\Gamma}$. First things first, considering transformation properties under the R-symmetry, the Fermi multiplet $\Theta$ inside the vector superfield may only be defined through $\overline{\mathcal{D}}_{+} \Theta=E_{\Theta}$ by the holomorphic function

$$
E_{\Theta}=[\Sigma, \tilde{\Sigma}]
$$

and by the superpotential $\mathcal{W}_{\Theta}=\tilde{\Phi} \Theta \Phi$, where $J_{\Theta}=\tilde{\Phi} \Phi$ is another holomorphic function.

On the contrary, the R-symmetry representations furnishing the $\mathcal{N}=(4,4)$ hypermultiplet, define its Fermi multiplets as

$$
E_{\Gamma}=\Sigma \Phi, \quad E_{\tilde{\Gamma}}=-\tilde{\Phi} \Sigma
$$

and let for the superpotential $\mathcal{W}_{\Gamma}+\mathcal{W}_{\tilde{\Gamma}}=\tilde{\Phi} \tilde{\Sigma} \Gamma+\tilde{\Gamma} \tilde{\Sigma} \Phi$, where $J_{\Gamma}=\tilde{\Phi} \tilde{\Sigma}$ and $J_{\tilde{\Gamma}}=\tilde{\Sigma} \Phi$.

In reality, it is not just the R-symmetry representations that we took into account to shape the above functions, but also the constraining condition $E \cdot J=\sum_{a} E_{a} J^{a}=0$ that should hold for supersymmetry to be preserved; of course, it is easy to see that this is satisfied for the given functions. The holomorphic functions $E_{a}$ and $J^{a}$ give the potential terms $\sim\left|E_{a}\left(\phi_{i}\right)\right|^{2}$ and $\sim\left|J_{a}\left(\phi_{i}\right)\right|^{2}$ in the action and produce an interesting interactive sector in our theory that is going to become decisively important in Section 5 .

\section{Dielectric branes on the boundary}

The case studied in 32,33 and in the previous section is dedicated to supergravity solutions defined by functions $h_{4}, h_{8}$ that vanish at the endpoints of the $\rho$-dimension, as in Figure 1.1 . Nevertheless, this is just one choice among many.

To classify all other possible kinds of solutions we must first consider the restrictions that apply on the functions $h_{4}, h_{8}$ and $u$. That is, these defining functions have to be chosen in such a way that the space properly closes on the $\rho$-dimension. Considering a linear $u$ function, 
both $h_{4}, h_{8}$ need to be zero at the $\rho=0$ endpoint whereas at $\rho=\rho_{f}$ only one of them needs to vanish. For a constant $u$ function, on the other hand, just one of them has to vanish at any endpoint. As we are about to find out, the physical set-up significantly changes depending on whether the function $u$ is linear or just a constant, both being legitimate solutions of the BPS equation $u^{\prime \prime}(\rho)=0$.

While all those novel cases are totally valid as supergravity solutions (i.e. they satisfy the equations of motion (1.3), a particular ambiguity arises in their dual quiver field theories. The ambiguity is that the gauge anomalies for these new quivers do not seem to cancel. In particular, it is the color nodes on the edges of the quivers that - naively - seem anomalous.

A promising answer to this riddle arises by focusing back on the supergravity side and observing the limiting geometry at the endpoints of the $\rho$-dimension (where the physics is dual to the aforementioned color nodes at the quiver edges). On those limiting vicinities, in contrast with the original paradigm of the previous section where the limiting space is either smooth or has O-planes, we now find D-branes. This is promising because explicit D-branes correspond to flavor symmetries (i.e. flavor nodes) that may contribute in the necessary way to cancel the gauge anomalies. Indeed, this is exactly what happens. But let us better realize all this through some solid examples. 


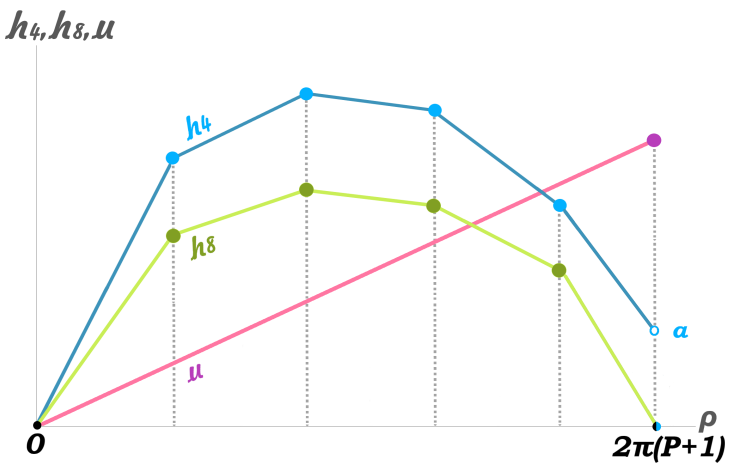

(a) A background with linear $u$ and a nonvanishing $h_{4}$ at the endpoint. $h_{4, h}, \boldsymbol{l}, \boldsymbol{l}$

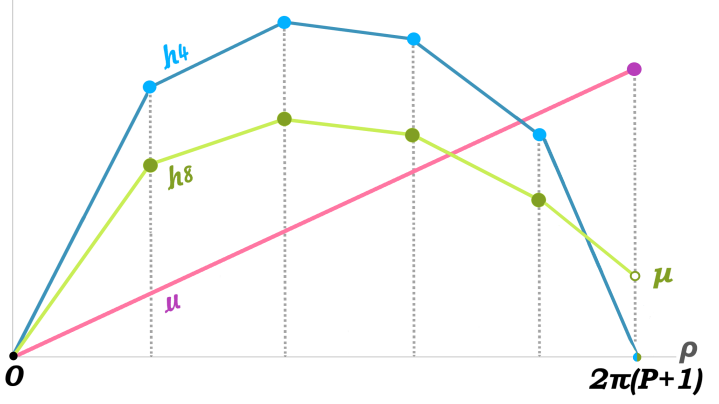

(b) A background with linear $u$ and a nonvanishing $h_{8}$ at the endpoint.

Figure 1.4: All the possible classes of backgrounds defined by a linear function $u(\rho)$ and a non-vanishing function $h_{4}$ or $h_{8}$ at the endpoint $\rho=\rho_{f}$.

\subsection{Linear $u(\rho)$}

As restated, the physics of the supergravity solutions changes depending on whether the function $u$ is linear or just a constant. Therefore, we split our analysis into two distinct parts, with regards to this property. The possible classes of backgrounds with linear $u$ and a non-vanishing $h_{4}$ or $h_{8}$ at the endpoint $\rho=\rho_{f}$ are classified in Figure 1.4 .

\section{Example I}

We begin by studying the class of backgrounds that is defined by a linear function $u$ and a non-vanishing function $h_{4}$ at the endpoint $\rho=\rho_{f}$, that is Figure 1.4a Nevertheless, because all the interesting action takes place in the last interval of the $\rho$-dimension (and its dual quiver gauge end-node) whose behavior we essentially care about, we shall study the simplest version of this class. That would be Figure $1.5 \mathrm{a}$.

The class of backgrounds represented by Figure $1.5 \mathrm{a}$ are defined by a linear function $u$ and by the functions

$$
\begin{aligned}
& h_{4}(\rho)=\left\{\begin{array}{cl}
\frac{\beta}{2 \pi} \rho & 2 \pi k \leq \rho \leq 2 \pi(k+1) \quad k=0, \ldots, P-1, \\
\alpha-\frac{\beta P-\alpha}{2 \pi}(\rho-2 \pi(P+1)) & 2 \pi P \leq \rho \leq 2 \pi(P+1)
\end{array}\right. \\
& h_{8}(\rho)=\left\{\begin{array}{cl}
\frac{\nu}{2 \pi} \rho & 2 \pi k \leq \rho \leq 2 \pi(k+1) \quad k=0, \ldots, P-1 . \\
\frac{\nu P}{2 \pi}(2 \pi(P+1)-\rho) & 2 \pi P \leq \rho \leq 2 \pi(P+1)
\end{array}\right.
\end{aligned}
$$




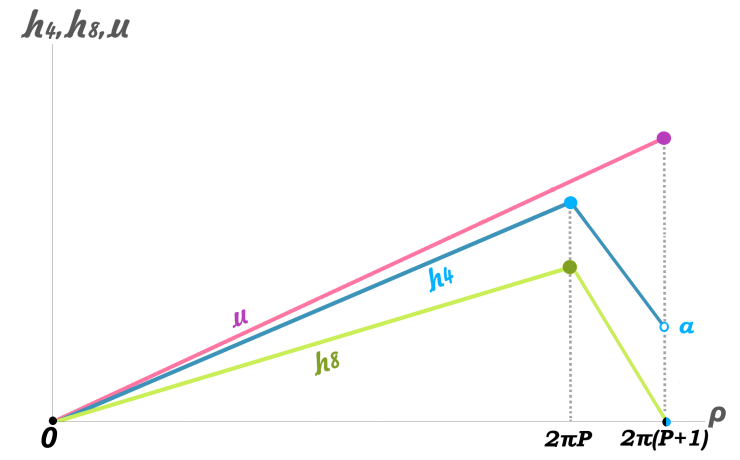

(a) A simplified version of Figure 1.4a. The function $u$ is linear, $h_{8}$ starts and closes with a vanishing value and $h_{4}$ vanishes at zero but not at $\rho=\rho_{f}$.

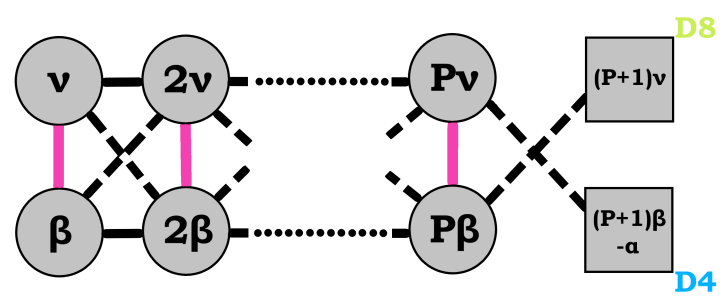

(b) This is the naive quiver dual to the background defined by (1.21), 1.22). In reality, there is one more flavor node, canceling the gauge anomalies for the last D6 gauge node.

Figure 1.5: A simplified version of the background given in Figure 1.4a and its dual quiver theory. Here, besides a linear function $u, h_{8}$ starts and closes with a vanishing value, while $h_{4}$ starts at zero but finishes at a non-zero value.

The background defined by these functions is - naively - dual to the quiver theory given by Figure $1.5 \mathrm{~b}$. The fact that this quiver is not the right one can be easily seen by observing the last D6 gauge node, i.e. the one with gauge rank $P \nu$; using the anomaly cancellation condition 1.13, the gauge anomalies on this node do not cancel. On the contrary, anomaly cancellation would occur if the gauge node was to connect with an additional flavor node of rank $\alpha$ through a $\mathcal{N}=(0,2)$ Fermi multiplet.

This raises a puzzle, since the standard Hanany-Witten brane set-up introduced in 32,33 (and represented by Figure 1.1) does not include any additional D-branes at the endpoints of the $\rho$-dimension, which would support such an additional flavor symmetry. Nonetheless, in contrast to that particular case, our solution defined by 1.21 and 1.22 has the novelty of a non-vanishing function $h_{4}$ at $\rho=\rho_{f}$. Hence, we shall focus on that vicinity of the supergravity background, which is dual to the problematic D6 gauge node, and see whether there is anything interesting there. That is, we focus near the end point $\rho=2 \pi(P+1)-x$, for $x \rightarrow 0$, where the geometry and the dilaton read

$$
\mathrm{d} s^{2}=\frac{1}{\sqrt{x}}\left(s_{1} \mathrm{~d} s_{\mathrm{AdS}_{3}}^{2}+s_{2} \mathrm{~d} s_{\mathrm{CY}_{2}}^{2}\right)+\sqrt{x}\left(s_{3} \mathrm{~d} x^{2}+s_{4} \mathrm{~d} s_{\mathrm{S}^{2}}^{2}\right), \quad e^{\phi}=s_{5} x^{-\frac{3}{4}}
$$

with $s_{i}$ real constants. As foreseen, we reached an interesting outcome since this background corresponds to D6-branes on $\mathrm{AdS}_{3} \times \mathrm{CY}_{2}$ and smeared over $\mathrm{S}^{2}$. To be exact, the above metric and dilaton also correspond to O6-planes, however only D6-branes can host open strings on their worldvolume and, thus, we only consider those to deduce global symmetries. That is, 
being explicit branes, these D6's contribute to the flavor structure of the quiver theory and, in principle, they should cancel the gauge anomalies on the last D6 gauge node.

On the other hand, the Bianchi identities yield no explicit D6-branes in our supergravity construction. According to the violation of these identities, the $h_{4}$ function - that appears here to feed the boundary of the space with D6-branes - may only give rise to D4-branes. Hence, since we do know we should have D4-branes at the endpoint where $h_{4}$ does not vanish, while we do not see them, we go on and study their sources. That is, we look upon their full Chern-Simons action 157

$$
\begin{aligned}
S_{\mathrm{CS}}^{\mathrm{D} 4} & =\mu_{4} \int \operatorname{Tr} \sum e^{i \lambda \imath_{\Phi} \imath_{\Phi}} C_{(n)} e^{\mathcal{F}_{2}} \\
& =\mu_{4} \int \operatorname{Tr} C_{5}^{e l}+C_{3}^{e l} \wedge \mathcal{F}_{2}+i \lambda\left(\imath_{\Phi} \imath_{\Phi}\right) C_{7}^{e l}-\lambda^{2}\left(\imath_{\Phi} \imath_{\Phi}\right)^{2}\left(C_{9}^{e l}+C_{7}^{e l} \wedge \mathcal{F}_{2}+\ldots\right),
\end{aligned}
$$

where the sum keeps only five-form terms that may source D4-branes. $C^{e l}$ is the electric part of a potential form, $\mathcal{F}_{2}=B_{2}+\lambda \tilde{f}_{2}$ is the gauge invariant field strength that incorporates the D4 worldvolume gauge field and $\imath_{\Phi}$ reflects the inner product with the D4-brane transverse modes $\Phi^{i}$. Dimensional analysis here implies $\lambda=2 \pi l_{s}^{2}$, up to a proportionality constant. The first term in the second line sources standard D4-branes, the second term reflects a D4/D2 bound state, while the third gives a D4/D6 bound state and so on. While the object $C_{3} \wedge \mathcal{F}_{2}$ realizes D2-charge induced into the D4-brane worldvolume, the seminal work by Myers 157 showed that an RR potential coupled to the transverse modes $\Phi^{i}$ represents a polarization of lower-dimensional D-branes into a higher-dimensional one.

Taking into account the RR fluxes of 1.2 and the functional forms $1.21,1.22$ near the endpoint $\rho \rightarrow \rho_{f}$, we pick a convenient gauge choice and deduce that

$$
\begin{aligned}
C_{3}^{e l}, C_{5}^{e l} & \rightarrow \text { const. } \quad C_{7}^{e l} \propto\left(\frac{-1}{\rho_{f}-\rho}\right) \operatorname{vol}\left(\mathrm{AdS}_{3}\right) \wedge \operatorname{vol}\left(\mathrm{CY}_{2}\right) \rightarrow-\infty \\
C_{9}^{e l} & \propto\left(\log \left(\rho_{f}-\rho\right)\right) \operatorname{vol}\left(\mathrm{AdS}_{3}\right) \wedge \operatorname{vol}\left(\mathrm{CY}_{2}\right) \wedge \operatorname{vol}\left(\mathrm{S}^{2}\right) \rightarrow-\infty
\end{aligned}
$$

Since $C_{7}^{e l}$ and $C_{9}^{e l}$ blow up at the boundary, then their corresponding source terms in the Chern-Simons action 1.24 dominate the game as opposed to the rest. Between those two potentials, $C_{7}^{e l}$ scales infinitely faster as we approach $\rho_{f}$ and therefore we argue that, at the boundary, the D4-branes couple to an infinitely strong $C_{7}^{e l} \mathrm{RR}$ potential and condense out 
into D6-branes, yielding the analogous background 1.23 . In fact, it should be the fifth term in the expansion of (1.24) that prevails; it is this particular term that yields bound states of D6-branes that are smeared over $S^{2}$ (under the coupling to $\mathcal{F}_{2}$ ), which agrees with the background (1.23). The third term in (1.24) gives just ordinary (not smeared) bound states of D6-branes. Finally, notice the fact that we have a non-vanishing $C_{5}^{e l}$; this is vital for the very existence of the constituent D4-branes on the D4/D6 bound state.

Recalling our original goal, we want to find the way this D4/D6 bound state contributes to the flavor symmetry of the theory. That is, the strings on the condensed D4-branes form a $\mathrm{U}\left(N_{4}\right)$ gauge theory under certain conditions, $N_{4}$ being the number of those branes given by the Bianchi identity

$$
\mathrm{d} \hat{f}_{4}=h_{4}^{\prime \prime} \mathrm{d} \rho \wedge \operatorname{vol}\left(\mathrm{CY}_{2}\right)
$$

The $\mathrm{U}\left(N_{4}\right)$ flavor gauge group is what we are after and anticipate of it canceling the gauge anomalies in the quiver theory.

To calculate (1.26) at the boundary, we have to handle things delicately. This is because the number of four-branes is associated with $h_{4}^{\prime}$ and a derivative is not well defined on the endpoint of a closed interval. Therefore, we shall demand that $\left.h_{4}\right|_{\rho_{f}}=0$, so that the derivative becomes well-defined near the endpoint $\rho_{f} \bigsqcup^{3}$. This is not a physical requirement of any short; it is just a trick to calculate the D-branes at the end of the space. Thus we now have the derivative

$$
\left.h_{4}^{\prime}\right|_{\rho \rightarrow \rho_{f}}=\lim _{x \rightarrow 0} \frac{h_{4}\left(\rho_{f}\right)-h_{4}\left(\rho_{f}-x\right)}{x}=\lim _{x \rightarrow 0} \frac{-\alpha}{x}
$$

and, in order to calculate all the four-branes on the endpoint, the D4 Page charge in (1.7) has to be integrated ${ }^{4}$ towards $\rho_{f}$ as

$$
N_{4}=-\int_{\rho_{f}-x}^{\rho_{f}} h_{4}^{\prime}=\alpha
$$

\footnotetext{
${ }^{2} \mathrm{~A}$ more elaborate proof of this is based in the string length $(\lambda-)$ order of those $C_{7}^{e l}$-terms and comes through the analogous case of the upcoming Section 3.2 , which is thoroughly analyzed in Appendix 1 . There, we will show that only terms of, at least, order $\mathcal{O}\left(\lambda^{2}\right)$ can provide non-trivial solutions for the D-brane bound states.

${ }^{3}$ The essence of differentiation is to realize how a function changes. In our particular context, the measure of this change is associated with the number of branes at a point. Since the background is defined on a closed interval, it makes sense to realize the absence of branes out of it as a shift of the defining function to a vanishing value. Stated otherwise, we exchange emptiness for a zero.

${ }^{4}$ The trick we applied on the $h_{4}$ function, forms a situation where the branes appear smeared near the endpoint, instead of being localized with a delta function as with the rest of the D4-brane stacks along the $\rho$ dimension. This is merely an artifact of our particular handling that is resolved just by adding up (integrating over) all the branes near that endpoint.
} 


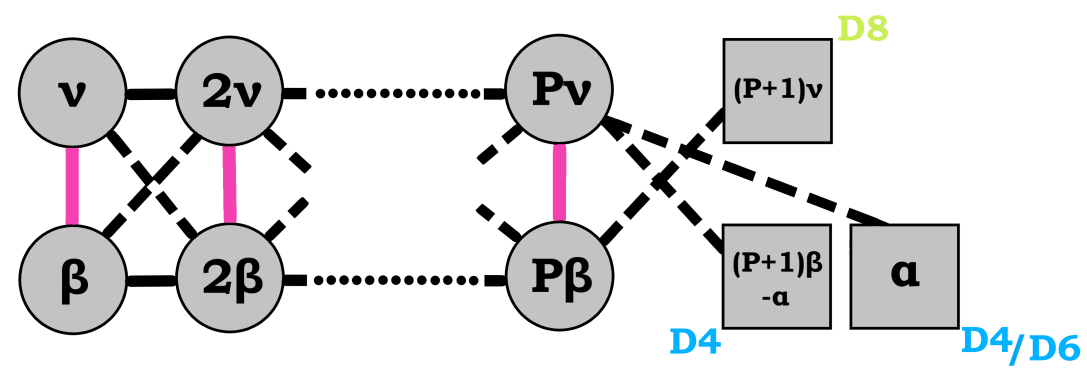

Figure 1.6: This is the actual quiver dual to the background defined by $(1.21),(1.22)$. Here, the extra four-brane flavor node cancels the gauge anomalies for the last $h_{8}(\mathrm{D} 6)$ gauge node.

Bottom line, we found $\alpha$ D4-branes sitting on the endpoint of the $\rho$-interval and being in a D4/D6 bound state.

The polarization that takes place should raise the question whether the D4-branes are enough in number, throughout the bound state, to support massless string modes and thus a unitary gauge theory. In reality, though, we are not obligated to know the precise geometry of the polarized branes, just that they are enough in number to be close to one another so that the modes do not get massive. And fortunately we do know that the D4-branes are a lot, since $\alpha$ must be large in the supergravity limit by construction. Therefore $\mathrm{U}(\alpha)$ should be the gauge group we have anticipated.

Being explicit branes, the worldvolume theory of those D4-branes feeds, through a $\mathcal{N}=(0,2)$ Fermi multiplet, the D6 color chain of the quiver with flavor. In particular, this $\mathrm{U}(\alpha)$ gauge group is dual to a global symmetry in the quiver theory which, using (1.13), gives exactly the flavor needed in order to cancel the gauge anomalies of the last D6 color chain node. This is all visualized in Figure 1.6, where the quiver theory is now consistent.

Focusing on the starting point $\rho=0$ of the $\rho$-interval, the background becomes the nonAbelian T-dual of $\mathrm{AdS}_{3} \times \mathrm{S}^{3} \times \mathrm{CY}_{2}$, which yields no D-branes there. This is to be expected from the supergravity side, since everything is obviously smooth there. But even by just looking at the field theory, the quiver is non-anomalous at its beginning (and now everywhere for that matter), which means that no additional D-branes should be there. If there were any, these would contribute with flavor and spoil the anomaly cancellation balance. 


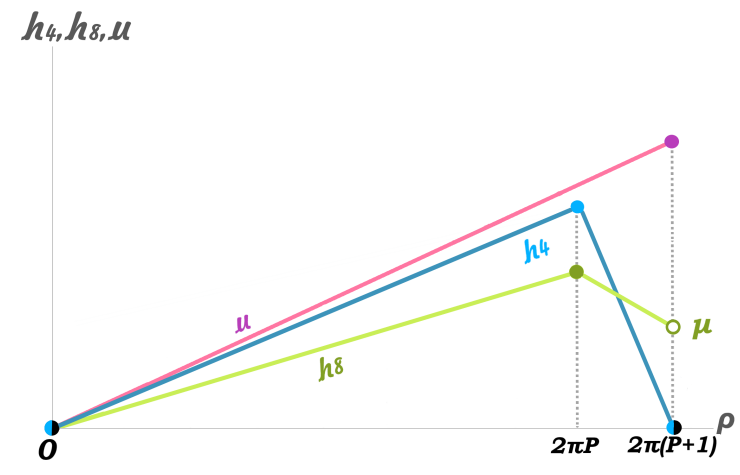

(a) A simplified version of Figure $1.4 \mathrm{~b}$. The function $u$ is linear, $h_{4}$ starts and closes with a vanishing value and $h_{8}$ vanishes at zero but not at $\rho=\rho_{f}$.

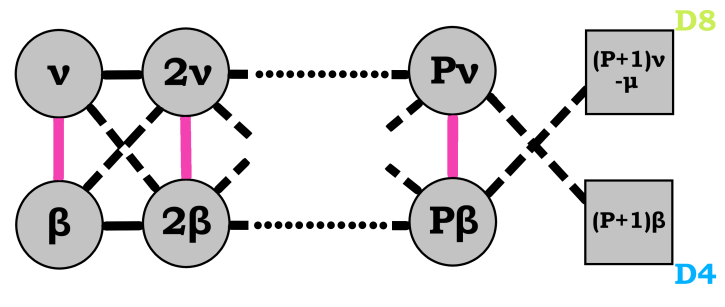

(b) This is the naive quiver dual to the background defined by $(1.29), 1.30)$. In reality, there is one more flavor node, canceling the gauge anomalies for the last D2 gauge node.

Figure 1.7: A simplified version of the background given in Figure 1.4b and its dual quiver theory. Here, besides a linear function $u, h_{4}$ starts and closes with a vanishing value, while $h_{8}$ starts at zero but finishes at a non-zero value.

\section{Example II}

Next, let us study the case represented by Figure 1.4b. Again, we consider Figure 1.7a instead which falls into the same class of backgrounds but is way simpler. This is the class of backgrounds where $h_{8}$ does not vanish at the end of the $\rho$-interval while $h_{4}$ does.

Therefore, according to Figure $1.7 \mathrm{a}$ the defining functions read

$$
\begin{aligned}
& h_{4}(\rho)=\left\{\begin{array}{cc}
\frac{\beta}{2 \pi} \rho & 2 \pi k \leq \rho \leq 2 \pi(k+1) \quad k=0, \ldots, P-1, \\
\frac{\beta P}{2 \pi}(2 \pi(P+1)-\rho) & 2 \pi P \leq \rho \leq 2 \pi(P+1)
\end{array}\right. \\
& h_{8}(\rho)=\left\{\begin{array}{cc}
\frac{\nu}{2 \pi} \rho & 2 \pi k \leq \rho \leq 2 \pi(k+1) \quad k=0, \ldots, P-1, \\
\mu-\frac{\nu P-\mu}{2 \pi}(\rho-2 \pi(P+1)) & 2 \pi P \leq \rho \leq 2 \pi(P+1)
\end{array}\right.
\end{aligned}
$$

The background defined by these functions is - naively - dual to the quiver theory given by Figure 1.7b. Again, this quiver cannot be the right one and this can be seen by using the anomaly cancellation condition 1.13 on the last D2 gauge node, i.e. the one with gauge rank $P \beta$. For that node the gauge anomalies do not cancel. On the contrary, anomaly cancellation would occur if it connected to a flavor node of rank $\mu$ through a $\mathcal{N}=(0,2)$ Fermi multiplet.

We go on and focus on the dual geometric vicinity of the 'anomalous' gauge node, an- 
ticipating again to find the necessary portion of D-branes that cancel the gauge anomalies. We find that near the endpoint, $\rho=2 \pi(P+1)-x$, for $x \rightarrow 0$, the backgrounds reads

$$
\mathrm{d} s^{2}=\frac{s_{1}}{\sqrt{x}} m_{1} \mathrm{~d} s_{\mathrm{AdS}_{3}}^{2}+\sqrt{x}\left(m_{2} \mathrm{~d} \rho^{2}+m_{3} \mathrm{~d} s_{\mathrm{S}^{2}}^{2}+m_{4} \mathrm{~d} s_{\mathrm{CY}_{2}}^{2}\right), \quad e^{\phi}=m_{5} x^{\frac{1}{4}},
$$

with $m_{i}$ real constants, which corresponds to D2-branes on $\mathrm{AdS}_{3}$ and smeared over $\mathrm{CY}_{2} \times \mathrm{S}^{2}$. To be exact, this background also corresponds to O2-planes, but strings may live only on D2-branes and, thus, we only consider those to search for global symmetries. Being explicit branes, these D2-branes contribute to the flavor structure of the quiver theory and, in principle, they should cancel the gauge anomalies.

However, we encounter the same problem as with Example I. That is, the Bianchi identities yield that the $h_{8}$ function only gives rise to D8-branes and certainly not to D2-branes. Therefore, since we do know we should have D8-branes at the endpoint $\rho=\rho_{f}$ where the $h_{8}$ function is non-vanishing, while we do not see them, we look up the D8-branes' source terms, that is their Chern-Simons action

$$
S_{\mathrm{CS}}^{\mathrm{D} 8}=\mu_{8} \int \operatorname{Tr} C_{9}^{e l}+C_{7}^{e l} \wedge \mathcal{F}_{2}+C_{5}^{e l} \wedge \mathcal{F}_{2} \wedge \mathcal{F}_{2}+C_{3}^{e l} \wedge \mathcal{F}_{2} \wedge \mathcal{F}_{2} \wedge \mathcal{F}_{2}
$$

where the first term sources standard D8-branes and the rest reflect eight-branes as bound states of D6, D4 and D2-branes, respectively. Here, we omitted the coupling to the single D8 transverse mode since there is no object into which this brane could possibly polarize.

Taking into account the RR sector 1.2 near the endpoint $\rho=\rho_{f}$, we again pick a convenient gauge and deduce

$$
\begin{gathered}
C_{7}^{e l}, C_{9}^{e l} \rightarrow \text { const. }, \quad C_{5}^{e l} \propto\left(\log \left(\rho_{f}-\rho\right)\right) \operatorname{vol}\left(\mathrm{AdS}_{3}\right) \wedge \operatorname{vol}\left(\mathrm{S}^{2}\right) \rightarrow-\infty \\
C_{3}^{e l} \propto\left(\frac{-1}{\rho_{f}-\rho}\right) \operatorname{vol}\left(\mathrm{AdS}_{3}\right) \rightarrow-\infty
\end{gathered}
$$

Since $C_{5}^{e l}$ and $C_{5}^{e l}$ blow up at the boundary, then their corresponding source terms in the Chern-Simons action 1.32 dominate the game as opposed to the rest. Between those two potentials, $C_{3}^{e l}$ scales infinitely faster as we approach $\rho_{f}$ and therefore we argue that, at the boundary, the D8-brane gauge field couples to an infinitely strong $C_{3}^{e l} \mathrm{RR}$ potential and 
induces D2-charge on its worldvolume, yielding the analogous background (1.31). Additionally, the smearing of those D2-branes can be understood by the coupling of $C_{3}^{e l}$ to $\left(\wedge \mathcal{F}_{2}\right)^{3}$, in the D8/D2 source term of 1.32 .

We conclude that the D8-branes' gauge field couples to D2-charge through the term

$$
S_{\mathrm{CS}}^{\mathrm{D} 8 / \mathrm{D} 2}=\frac{\mu_{2}}{(2 \pi)^{3}} \int \operatorname{Tr} C_{3}^{e l} \wedge \tilde{f}_{2} \wedge \tilde{f}_{2} \wedge \tilde{f}_{2}
$$

together forming a D8/D2 bound state. The D8 gauge flux on $\mathrm{CY}_{2} \times \mathrm{S}^{2}$ should be quantized as

$$
\frac{1}{(2 \pi)^{3}} \int_{\mathrm{CY}_{2} \times \mathrm{S}^{2}} \tilde{f}_{2} \wedge \tilde{f}_{2} \wedge \tilde{f}_{2}=N_{2} \quad \text { for } \quad N_{2} \in \mathbb{Z}
$$

and the D2-branes are explicitly given by the Bianchi identity

$$
\mathrm{d} \hat{f}_{6}=\frac{\lambda^{3}}{3 !} \tilde{f}_{2} \wedge \tilde{f}_{2} \wedge \mathrm{d} F_{0}=\frac{\lambda^{3}}{3 !} N_{2} \operatorname{vol}\left(\mathrm{CY}_{2}\right) \wedge \operatorname{vol}\left(\mathrm{S}^{2}\right) \wedge\left(h_{8}^{\prime \prime} \mathrm{d} \rho\right)
$$

Hence, we conclude that every eight-brane on the boundary should exist exclusively in a D8/D2 bound state, sourced by

$$
S_{\mathrm{CS}}^{1 \times \mathrm{D} 8 / \mathrm{D} 2}=N_{2}\left(\mu_{2} \int C_{3}^{e l}\right)
$$

that is each D8-brane contains $N_{2}$ units of D2-charge.

Nonetheless, there is no just one D8-brane (with an Abelian gauge field) but there should be multiple coincident D8-branes at the boundary. The number of these branes is given by the Bianchi identity

$$
\mathrm{d} \hat{F}_{0}=h_{8}^{\prime \prime} \mathrm{d} \rho
$$

where, following the same procedure for $h_{8}^{\prime}$ as in Example I with $h_{4}^{\prime}$, we find that at the boundary $\rho=\rho_{f}$ they amount to

$$
\left.N_{8}\right|_{\rho=\rho_{f}}=\mu
$$




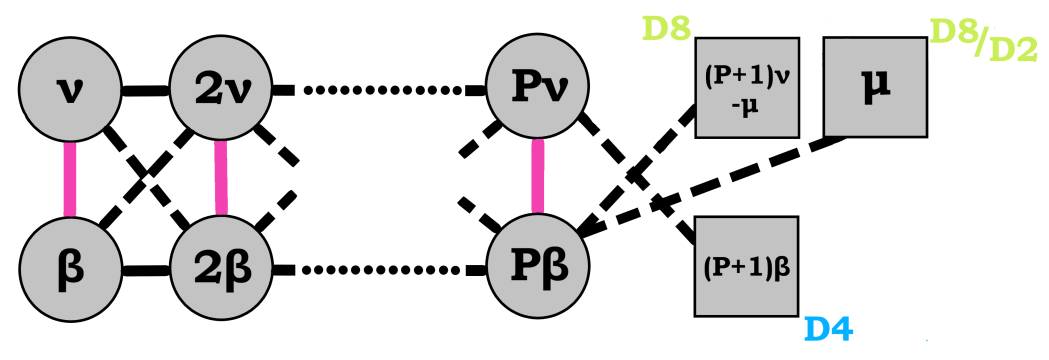

Figure 1.8: This is the actual quiver dual to the background defined by $(1.29),(1.30)$. Here, the extra D2 and D6 flavor nodes cancel the gauge anomalies for the first D6 and the last D2 gauge nodes.

Since those D8-branes are coincident and thus their gauge field is non-Abelian, a $\mathrm{U}(\mu)$ gauge theory arises that is realized as a global symmetry in the dual quiver theory and which should cancel the apparent gauge anomalies there.

Indeed, the D8-branes, as D8/D2 bound states, feed with flavor the end of the D2 color chain of the quiver through a $\mathcal{N}=(0,2)$ Fermi multiplet, as usual. As expected, using the anomaly cancellation condition (1.13), they give exactly the flavor needed in order to cancel the gauge anomalies of the last D2 node. This is all visualized in Figure 1.8, where the quiver theory is now consistent.

\subsection{Constant $u(\rho)$}

The class of supergravity backgrounds with constant function $u(\rho)$ is analogous but, at the same time, dissimilar to the linear case. The representative kinds of backgrounds in this class are the ones presented in Figures 1.9, distinguished by their constant $u(\rho)$ curve. Instead of going through both examples again, we now combine them into one that includes all the interesting behavior. That is, at the beginning of the $\rho$-dimension $h_{4}$ does not vanish while $h_{8}$ does, the opposite being true at the other endpoint. Of course, we again realize simplified versions of these cases as in the previous examples and, depending on the behavior of the defining functions at each endpoint, the precise form of $h_{4}$ and $h_{8}$ can be read off from (1.21), 1.22 and 1.29, 1.30). Accordingly, for this new background, we seek for $\mathrm{U}(\alpha)$ and $\mathrm{U}(\mu)$ flavor symmetries at $\rho=0$ and $\rho=\rho_{f}$ respectively, in order to cure the apparent gauge anomalies at the dual edge-nodes of the quiver chain. 


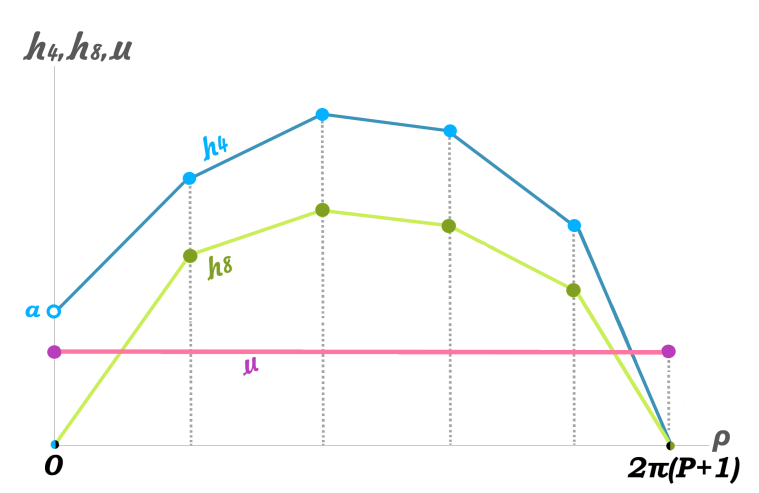

(a) A background with constant $u$ and a nonvanishing $h_{4}$ at the beginning $\rho=0$.

\section{$h_{4, h}, \boldsymbol{l}, \boldsymbol{l}$}

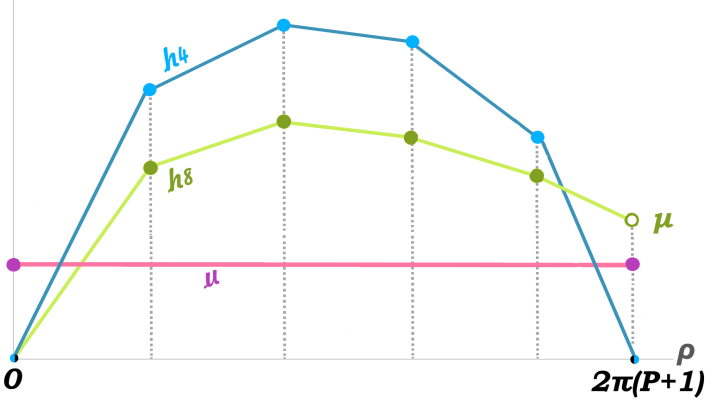

(b) A background with constant $u$ and a nonvanishing $h_{8}$ at the endpoint $\rho=\rho_{f}$.

Figure 1.9: The representative backgrounds defined by a constant $u(\rho)$ and a non-vanishing $h_{4}$ or $h_{8}$ at either endpoint. The roles of $h_{4}$ and $h_{8}$ may be exchanged in (a) and (b).

\section{At the beginning of the $\rho$-dimension}

The background we consider begins on its $\rho$-dimension, for $\rho=x$ while $x \rightarrow 0$, with a vanishing $h_{8}$ but a non-vanishing $h_{4}$ function, giving

$$
\mathrm{d} s^{2}=\frac{1}{\sqrt{x}}\left(m_{1} \mathrm{~d} s_{\mathrm{AdS}_{3}}^{2}+m_{2} \mathrm{~d} s_{\mathrm{S}^{2}}^{2}+m_{3} \mathrm{~d} s_{\mathrm{CY}_{2}}^{2}\right)+m_{4} \sqrt{x} \mathrm{~d} x^{2}, \quad e^{\phi}=m_{5} x^{-\frac{5}{4}}
$$

that corresponds to D8-branes on $\mathrm{AdS}_{3} \times \mathrm{S}^{2} \times \mathrm{CY}_{2}$, which again seems odd since $h_{4}$ only gives D4-branes. Our experience gained from the precious sections drives us to study the full Chern-Simons source action of $N_{4}$ D4-branes, including the coupling of the transverse string modes to the higher dimensional RR fields, as

$$
S_{\mathrm{CS}}^{D 4}=\mu_{4} \int \operatorname{Tr} C_{5}^{e l}+C_{3}^{e l} \wedge \mathcal{F}_{2}+i \lambda\left(\imath_{\Phi} \imath_{\Phi}\right) C_{7}^{e l}-\lambda^{2}\left(\imath_{\Phi} \imath_{\Phi}\right)^{2}\left(C_{9}^{e l}+C_{7}^{e l} \wedge \mathcal{F}_{2}+\ldots\right)
$$

where the first term represents standard D4-branes and the second D4/D2 bound states, while the rest reflect polarized D4-branes into higher dimensional ones. Considering the RR sector $(1.2)$ near the beginning $\rho=0$, we deduce

$$
C_{3}^{e l}, C_{7}^{e l} \rightarrow 0, \quad C_{5}^{e l} \rightarrow \text { const. }, \quad C_{9}^{e l} \rightarrow-\infty
$$


at the vicinity of that boundary, where again a convenient gauge was chosen.

Therefore, at $\rho \rightarrow 0$, only the first and fourth term survive in (1.41), which stand for standard D4-branes and D4/D8 bound states, respectively. Since the potential $C_{9}^{e l}$ blows up, without any competition this time, the fourth term in the above action dominates the first and this is why the background metric and dilaton behave according to 1.40 . That is, the D4-branes couple to an infinitely strong $\mathrm{RR}$ potential $C_{9}^{e l}$ and condense out into an eight-brane, forming a D8/D4 bound state while giving a D8-brane background on that boundary. Of course, the non-vanishing $C_{5}^{e l}$ is vital for the very existence of those constituent D4-branes. As it is the case with Example I and (1.24), both the coupling to the transverse scalars and the string length order in the Chern-Simons action (1.41) would make here a more detailed treatment instructive, a calculation that is held in Appendix I.

Casting the usual trick on $h_{4}^{\prime}$, we count $\alpha$ D4-branes on $\rho=0$, on which open strings end and make up a $\mathrm{U}(\alpha)$ gauge theory. The polarization that takes place over $\mathrm{CY}_{2}$ should raise the question whether the D4-branes are enough in number, throughout the bound state, to support massless string modes and thus a unitary gauge theory. As restated though, we do know that the D4-branes are a lot since $\alpha$ must be also large in the supergravity limit, by construction. Therefore $\mathrm{U}(\alpha)$ is the flavor group we anticipated for the beginning node of the quiver chain, canceling exactly the gauge anomalies there through a $\mathcal{N}=(0,2)$ Fermi multiplet.

\section{At the end of the $\rho$-dimension}

Focusing on the other endpoint, $\rho=2 \pi(P+1)-x$ while $x \rightarrow 0$, the same background ends on its $\rho$-dimension with a vanishing $h_{4}$ but a non-vanishing $h_{8}$, giving

$$
\mathrm{d} s^{2}=\frac{1}{\sqrt{x}}\left(s_{1} \mathrm{~d} s_{\mathrm{AdS}_{3}}^{2}+s_{2} \mathrm{~d} s_{\mathrm{S}^{2}}^{2}\right)+\sqrt{x}\left(s_{3} \mathrm{~d} x^{2}+s_{4} \mathrm{~d} s_{\mathrm{CY}_{2}}^{2}\right), \quad e^{\phi}=s_{5} x^{-\frac{1}{4}},
$$

which corresponds to D4-branes smeared over $\mathrm{CY}_{2}$. While this seems odd since $h_{8}$ only produces D8-branes, our wisdom off the previous section guides us to study the source terms

$$
S_{\mathrm{CS}}^{\mathrm{D} 8}=\mu_{8} \int C_{9}^{e l}+C_{7}^{e l} \wedge \mathcal{F}_{2}+C_{5}^{e l} \wedge \mathcal{F}_{2} \wedge \mathcal{F}_{2}+C_{3}^{e l} \wedge \mathcal{F}_{2} \wedge \mathcal{F}_{2} \wedge \mathcal{F}_{2}
$$

where the first term sources a standard D8-brane and the rest reflect a D8-brane in a bound 
state with D6, D4 and D2-branes, respectively.

Studying the RR fluxes (1.2) at $\rho \rightarrow \rho_{f}$ for a constant function $u$ again, the potentials behave as

$$
C_{7}^{e l} \rightarrow 0, \quad C_{3}^{e l}, C_{9}^{e l} \rightarrow \text { const. }, \quad C_{5}^{e l} \rightarrow-\infty,
$$

where we again chose a convenient gauge. The fact that $C_{7}^{e l}$ vanishes excludes the D8/D6 bound state whatsoever. Between the rest of the terms in 1.44 , the one that couples to $C_{5}^{e l}$ dominates since it is this potential that blows up at the vicinity of that endpoint.

We conclude that the D8-brane gauge field couples to D4-charge through the term

$$
S_{\mathrm{CS}}^{\mathrm{D} 8 / \mathrm{D} 4}=\frac{\mu_{4}}{4 \pi^{2}} \int \operatorname{Tr} C_{5}^{e l} \wedge \tilde{f}_{2} \wedge \tilde{f}_{2}
$$

together forming a D8/D4 bound state. The fact that $C_{5}^{e l}$ is infinitely strong makes the source term 1.46 dominant in (1.44) and this is why the eight-branes are geometrically realized as smeared D4-branes. The D8 gauge flux on $\mathrm{CY}_{2}$ should be quantized as

$$
\frac{1}{4 \pi^{2}} \int_{\mathrm{CY}_{2}} \tilde{f}_{2} \wedge \tilde{f}_{2}=N_{4} \quad \text { for } \quad N_{4} \in \mathbb{Z}
$$

and the D4-branes are explicitly given by the Bianchi identity

$$
\mathrm{d} \hat{f}_{4}=\frac{\lambda^{2}}{2} \tilde{f}_{2} \wedge \tilde{f}_{2} \wedge \mathrm{d} F_{0}=\frac{\lambda^{2}}{2} N_{4} \operatorname{vol}\left(\mathrm{CY}_{2}\right) \wedge\left(h_{8}^{\prime \prime} \mathrm{d} \rho\right)
$$

Hence, we conclude that every eight-brane on the boundary should exist exclusively in a D8/D4 bound state, sourced by

$$
S_{\mathrm{CS}}^{1 \times \mathrm{D} 8 / \mathrm{D} 4}=N_{4}\left(\mu_{4} \int C_{5}^{e l}\right)
$$

that is each D8-brane contains $N_{4}$ units of D4-charge.

Nonetheless, there is no just one D8-brane but there should be multiple coincident D8branes at the boundary. The number of these branes, same as in the last section with Example II, is given by $N_{8}=\mu$. Since those D8-branes are coincident and thus their gauge field is non-Abelian, a $\mathrm{U}(\mu)$ gauge theory arises that is realized as a global symmetry in the dual field theory and which cancels exactly the gauge anomalies in the end of the quiver 
chain through a $\mathcal{N}=(0,2)$ Fermi multiplet.

Note that the smeared D4 and the D8-branes in this section are backgrounds equivalent to smeared O4 and O8-planes, respectively. Of course, strings may only live on the former which is why we only consider those to find the desired flavor symmetries.

As a last remark on the whole section, let us clarify a few details about the RR potentials. Firstly, the fact that we chose a particular gauge does not change any of the results. Indeed, by studying the RR fluxes we realize that had we picked any other gauge choice would have made no difference; the qualitative relationship between the $C_{p}$ forms (which one is stronger at the endpoints) would have stayed the same. Secondly, one may wonder whether such objects blowing up test the supergravity approximation. However, as argued in [33], singularities are bound to exist when D-branes do, while they are not dangerous as long as they are regulated and stay far apart from each other (here, along the $\rho$-dimension). This is exactly the case with the Ricci scalar (which diverges at the positions of localized sources) and with the RR potentials, as long as $\beta_{k}, \nu_{k}, P$ are large. Indeed, large $\beta_{k}, \nu_{k}$ control all divergences, while large $P$ keeps the singularities far apart (for the backgrounds we considered, RR potentials only blow up at the endpoints, anyway). Nonetheless, we believe that the particular divergence of some of the RR potentials at the endpoints is an artifact of the functions $h_{4}, h_{8}$ being defined on a closed interval; this was the case when we counted D-branes at those endpoints, where we had to go around the fact that $h_{4}^{\prime}, h_{8}^{\prime}$ are not well-defined there. The essence of those infinities in our context is that some potentials are profoundly stronger than others.

Aside from curing a problem and better realizing the way the dual field theory works, this section has an additional value. Since the discovery of particular flavor branes was the exact thing that made the quiver theory consistent, this calculation provides an additional validity check of the whole field theoretical structure. Further validation of the quantum quiver structure is especially important here, since the matter content of these quiver theories is by no means trivial. This is the subject of the following section.

\section{Adding matter in the quiver field theory}

The quantum quiver theory dual to the $\mathrm{AdS}_{3}$ supergravity vacua we consider was presented in Section 2.3. In 33 these linear-quiver theories were thoroughly analyzed and tested, while our previous section suits as further validation. Nevertheless, there is more to their story to tell. That is they are ultimately characterized by additional structure.

Let us address the problem in a constructive way. In a Hanany-Witten brane set-up, we have all possible kinds of oscillating strings stretched between the branes. In the dual quiver theory, these kinds of strings correspond to supersymmetric multiplets that bind the gauge 
theories (gauge nodes) together and constitute the matter content of the overall field theory. Thus, when we try to build the correct dual field theory of a particular kind of brane set-up, the problem boils down to finding all the possible matter content.

Establishing the quiver theory introduced in 32,34 as a well tested structure, we realize that there are two kinds of superfield connection missing. These are the multiplets connecting D2 gauge with D4 flavor nodes and the ones connecting D6 gauge with D8 flavor nodes, respectively representing D2-D4 and D6-D8 strings. Instead of quantizing, we may just ask what multiplets can possibly fill this gap. The problem gets quickly simplified, since we know we do not want to consider additional $\mathcal{N}=(0,4)$ hyper multiplets nor $\mathcal{N}=(0,2)$ Fermi multiplets. This is because their presence would spoil the fragile balance of the gauge anomaly cancellation once and for all, a balance that was further confirmed to holographically hold by the last section. Therefore, we should only consider $\mathcal{N}=(4,4)$ hyper multiplets.

Nonetheless, our unique choice should be in harmony with the central charge of the field theory. In particular, since the central charge was found in 33 to be holographically correct for the (original) quiver theory, then the new matter content we want to add should change nothing and be entirely invisible to it. Indeed, this is exactly the case. The central charge of the quiver field theory reads

$$
c=6\left(n_{\text {hyp }}-n_{\text {vec }}\right)=6\left(\sum_{j=1}^{P}\left(\alpha_{j} \mu_{j}-\alpha_{j}^{2}-\mu_{j}^{2}+2\right)+\sum_{j=1}^{P-1}\left(\alpha_{j} \alpha_{j+1}+\mu_{j} \mu_{j+1}\right)\right)
$$

which means that it is sensitive to the number of the hyper multiplets. This may sound discouraging wrt adding new $\mathcal{N}=(4,4)$ hyper multiplets, since we want to leave the central charge intact, but it is not. This is because we work in the supergravity limit, i.e. for $P \rightarrow \infty$, which means that we are eligible to add new hyper multiplets as long as their number is sub-leading in $P$ wrt to the old ones.

In the supergravity limit the sources (flavor nodes) should exist far apart along the linear quiver, which means that the new hyper multiplets escorting them are much less than the old ones that exist between the flavor positions (connecting the gauge nodes). The proposed, enhanced quiver theory is visualized in Figure 1.10.

In order to prove that the new hyper multiplets are always of lower order in $P$ than the old ones, we expand the already existing ones as

$$
n_{\text {hyp }}=\sum_{j=1}^{P}\left(\sum_{k=0}^{j-1} \beta_{k} \cdot \sum_{l=0}^{j-1} \nu_{l}\right)+\sum_{j=1}^{P-1}\left[\left(\sum_{k=0}^{j-1} \beta_{k} \cdot \sum_{l=0}^{j} \beta_{l}\right)+\left(\sum_{k=0}^{j-1} \nu_{k} \cdot \sum_{l=0}^{j} \nu_{l}\right)\right]
$$




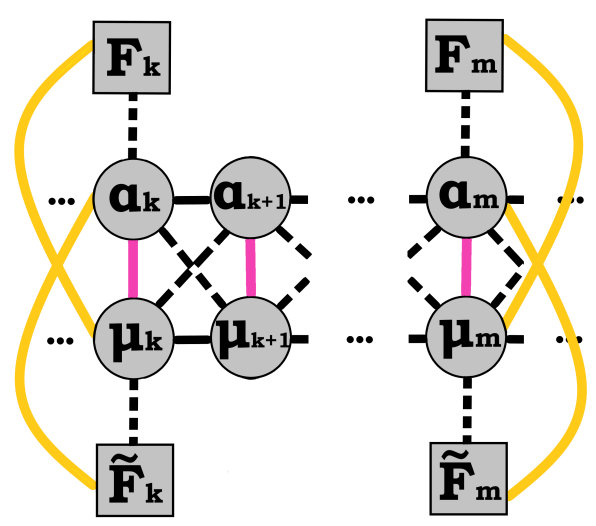

Figure 1.10: This is the new dual quiver theory, with additional $\mathcal{N}=(4,4)$ hyper multiplets binding the D4 and D8 flavor nodes with the D2 and D6 gauge nodes, respectively. The already existing $\mathcal{N}=(4,4)$ hyper multiplets are represented with black solid lines, while the new additional ones with orange solid lines.

while the new ones, $n_{\text {hyp }}^{\star}$, read

$$
\begin{aligned}
n_{h y p}^{\star} & =\sum_{j=i_{1}}^{i_{M}} \alpha_{j} \tilde{F}_{j-1}+\sum_{j=i_{1}}^{i_{N}} \mu_{j} F_{j-1} \\
& =\sum_{j=i_{1}}^{i_{M}}\left(\sum_{k=0}^{j-1} \beta_{k}\left(\beta_{j-1}-\beta_{j}\right)\right)+\sum_{j=i_{1}}^{i_{N}}\left(\sum_{k=0}^{j-1} \nu_{k}\left(\nu_{j-1}-\nu_{j}\right)\right),
\end{aligned}
$$

where $j=i_{1}, \ldots, i_{M, N}$ are the $M, N$ intervals with sources for the D4 and D8 branes, respectively. The fact that in the supergravity limit the sources (flavor nodes) should exist far apart along the linear quiver means $M, N \ll P$.

In order to compare $n_{\text {hyp }}$ and $n_{\text {hyp }}^{\star}$ we can just focus into similar terms between them. These are, for instance, the second term of 1.51 and the first of 1.52 . For them, we observe that their first summation is to $P-1$ and $i_{M}$, respectively. Since $M, N \ll P$, this means that the former is of order $P$ while the latter is not. Focusing on the inner summations of the same terms, we realize that their summing products are of the same order, whatever that is. Therefore, overall, $n_{\text {hyp }}$ is always an order higher in $P$ than $n_{\text {hyp }}^{\star}$, which makes the latter invisible in the central charge for $P \rightarrow \infty$.

The whole situation would be immediately cleared out if we quantized the system of Dbranes. What is more, quantizing the D2-D4 and D6-D8 systems in flat space seems to indeed reproduce the new $\mathcal{N}=(4,4)$ hypermultiplets that we just proposed to exist. However, this particular Hanany-Witten set-up is assumed to live in $\mathrm{CY}_{2}$ dimensions as well, which makes the standard quantization techniques obscure in the case at hand and, therefore, such a study 
remains on the sidelines at this point.

Another link that we intentionally left out is the multiplet corresponding to superstrings between D4 and D8 flavor branes. Not giving gauge groups, these links are allowed to be any multiplet as far as the gauge anomaly balance is concerned. Therefore, this situation demands to be properly quantized and thus eludes the present work.

Truth be told, there is another path through which we might have imagined that the additional matter is an essential ingredient to our theory. This argument too surfaces from the supergravity side of the duality, but in order to illustrate it we need to consider a particular state of the bosonic string. This is what we deal with in the following section.

\section{The meson string}

Having worked out even the most exotic parts of the duality between the massive IIA vacua and the dual quantum field theory, we are certainly in desire of testing their holographic performance. In that vein, we look for a simple object to construct, starting off with the supergravity side of the story.

\subsection{A BPS state}

The most accessible state in our theory of gravity is a semiclassical string stretching between D-branes. That is, we consider a meson string soliton $\mathcal{M}_{k, m}$ on the supergravity background, that extends between stacks of flavor branes at $\rho=2 \pi k$ and $\rho=2 \pi m$, respectively, and which is a point on the rest of the dimensions sitting at the center $r=0$ of $\mathrm{AdS}_{3}$. An analogous calculation was performed in [158].

Therefore, we allow a string embedding with $\tau=t, \sigma=\rho$, whose mass is essentially its length

$$
M_{\mathcal{M}}=\frac{1}{2 \pi} \int \mathrm{d} \sigma \sqrt{-\operatorname{det} g_{a b}}=\frac{1}{2 \pi} \int_{2 \pi k}^{2 \pi m} \mathrm{~d} \rho \sqrt{-\operatorname{det} g_{a b}}=m-k
$$

where $g_{a b}$ is the worldsheet pullback of the metric in (1.1). If $F_{k}$ and $F_{m}$ are the number of D-branes in the respective stacks on which the string endpoints are, then this configuration transforms in the bi-fundamental representation of $\mathrm{SU}\left(F_{k}\right) \times \mathrm{SU}\left(F_{m}\right)$.

Since we are always interested in states that preserve some supersymmetry, we may upgrade the above configuration to a BPS state just by considering the suspended string to fluctuate on the two-sphere, whose $\mathrm{SU}(2)$ isometry corresponds to the dual R-symmetry. This is done by including $\phi=\omega \tau$ in the above configuration, where we let this fluctuation to be small - i.e. $\omega \ll 1-$ so that the embedding simplifies still into the expression (1.53). Note, of course, that small $\omega$ does not imply small angular momentum $J_{R}$ for the semiclassical 
string; after all, $J_{R} \sim M$ for a BPS state. Indeed, as we show below, small angular velocity $\omega$ of the large semiclassical soliton reflects large angular momentum.

Picking a $\mathrm{U}(1)_{R}$ inside $\mathrm{SU}(2)_{R}$, we now seek the R-charge of the above state. Since the generator of the $\mathrm{U}(1)$ on the two-sphere is associated to the 1 -form $\cos \theta \mathrm{d} \phi$, then we look for the string coupling terms

$$
S_{R} \propto \int \cos \theta \mathrm{d} \phi
$$

As far as the R-charge is concerned, it may be read off the source terms of the form $\int J_{R} A_{1}=$ $Q_{R} \int A_{1}$, with $A_{1}=\cos \theta \mathrm{d} \phi$. The relevant term in the worldsheet action is

$$
S_{\mathcal{M}}=\frac{1}{2 \pi} \int_{\Sigma} B_{2}
$$

where $\Sigma=[2 \pi k, 2 \pi m] \times \mathbb{R}$. Ultimately, after some manipulation given in Appendix J this term may be actually seen as the source term

$$
S_{\mathcal{M}}=(m-k) \int_{\mathbb{R}} \cos \theta \mathrm{d} \phi,
$$

which yields an R-charge

$$
Q_{R}=m-k
$$

Comparing this with the string mass in (1.53), we conclude that this is indeed a BPS state.

\subsection{An ultraviolet operator}

Now, we want to look for the operator dual to this BPS state. To this end - since the IR SCFT is completely unknown - we consider the UV quiver theory on the $\rho$-interval $[2 \pi k, 2 \pi m]$ and pick the appropriate field excitations inside the supersymmetric multiplets.

Since we are dealing with a purely bosonic state, we are immediately led to consider the complex scalars $\phi_{i}$ inside the $\mathcal{N}=(0,2)$ chiral multiplets $\Phi_{i}$, since these are the obvious on-shell bosonic degrees of freedom in our theory. In particular, we choose to excite one scalar in each of the $(m-k)+2 \mathcal{N}=(4,4)$ hypermultiplets that connect two flavor nodes; this makes a perfect fit with the fact that string fluctuations transverse to the worldvolumes of branes are also scalar modes wrt these worldvolume theories. It also illustrates why we need the additional $\mathcal{N}=(4,4)$ matter, as promised in the beginning of this section; if it 


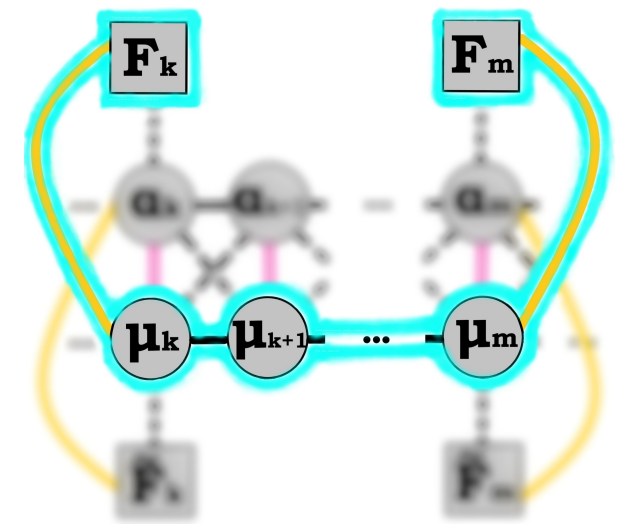

Figure 1.11: The meson operator $\mathcal{M}$ consists of the supersymmetric multiplets that are highlighted with blue, while the rest of the quiver structure is left blurred. If $k$ and $m$ are the positions of the flavor nodes along the quiver chain, then this operator runs over $m-k+2$ $\mathcal{N}=(4,4)$ hypermultiplets and $m-k+1 \mathcal{N}=(4,4)$ vector multiplets. Such an operator may also connect D4 with D8 flavors, by jumping through $\mathcal{N}=(0,4)$ hypermultiplets.

was not for these new hypermultiplets, there would be no way to build a string of bosonic field excitations that connect two flavor nodes. And such a dual bosonic connection must somehow exist, given that the meson string we consider is a legitimate BPS state.

Shortly, however, we spot a problem. As illustrated in Appendix H.2, the $\phi_{i}$ scalars inside any of the $\mathcal{N}=(0,4)$ hypermultiplets are uncharged under R-symmetry, while we do need an R-charge - according to (1.57), proportional to $(m-k)$ - for our proposed operator. In fact, the only scalars that are charged under the $\mathrm{U}(1)_{R}$ subgroup of the R-symmetry are the ones in the $\mathcal{N}=(0,4)$ twisted hypermultiplets $\left(\Sigma_{i}, \tilde{\Sigma}_{i}\right)$, inside the $\mathcal{N}=(4,4)$ vector superfields of the gauge nodes. This leads us to consider these scalars, let us call them $\sigma_{i}$, as well. The inclusion of these scalar fields is also somewhat compelling, since these are the ones that let the $\phi_{i}$ scalars interactively talk to each other; this realizes an interactive continuance among the string of fields in the operator, holographically analogous to the compactness of the string. These supersymmetric interactions will become apparent shortly.

All in all, choosing a $\sigma_{i}$ excitation as well in each gauge node between the $\mathcal{N}=(4,4)$ hypermultiplets, we acquire the meson operator

$$
\mathcal{M}_{k, m}=\pi_{k}\left(\prod_{i=k}^{m-1} \sigma_{i} \phi_{i}\right) \sigma_{m} \tilde{\pi}_{m}
$$

which transforms in the bifundamental representation of $\mathrm{SU}\left(F_{k}\right) \times \mathrm{SU}\left(F_{m}\right)$, with $F_{k}$ and $F_{m}$ the ranks of the flavor groups in the corresponding positions of the quiver chain. Here we named $\pi_{i}$ the scalars inside the end-point hypermultiplets connecting to the flavor nodes and also chose them to be in conjugate representations of each gauge group. Such an operator 
has two $\pi_{i}$ 's, $(m-k) \phi_{i}$ 's and $(m-k+1) \sigma_{i}$ 's, which in the supergravity limit - where sources are far apart - account for $2(m-k)$ complex scalars. Since only half of those (the $\sigma_{i}$ 's) are R-charged, this is the desired R-charge considering the BPS string charge (1.57). For clarity, the operator is highlighted in Figure 1.11 .

The only quantities left to compare are the mass 1.53 of the BPS state and the conformal dimension of the operator $\mathcal{M}_{k, m}$. At this point, of course, we may have an actual problem; scalar fields in two dimensions have mass dimension zero. At least classically. At first sight, this degrades our proposal for the operator which seems to have a vanishing scaling dimension. However, before rushing into conclusions, we remind ourselves that we have actually considered the UV operator and not the actual IR situation; it is the IR operator the one that should necessarily acquire the appropriate scaling dimension. Therefore, if the choice of operator is correct, our only way out is the possibility of the operator acquiring an anomalous dimension through quantum effects. Whatever the case is with the IR SCFT, such quantum effects should be present in the UV Lagrangian, pointing towards an anomalous dimension $\gamma(g)$ that scales with energy.

On the other hand, studying quantum corrections is obscure in our case. This is exactly because it is the UV theory that we use to organize fields into an operator; therefore even if we assume a completely anomalous dimension $\Delta_{\mathcal{M}}=\gamma(g)$, our SCFT is assumed to be strongly coupled which discredits any perturbative calculation. To be exact, it is the non-integrability of our $\mathrm{AdS}_{3}$ backgrounds 2 that prohibits surfing along the range of the coupling constant, as it is possible with e.g. the work of BMN 22] in the $\mathrm{AdS}_{5} \times \mathrm{S}^{5}$ correspondence. Regardless, the possibility itself of a non-perturbative anomalous dimension requires certain interactions to be there, between the fields of interest; finding whether those exist is essential to our proposal. Interestingly, such interactions indeed exist.

The interactions between the $\phi_{i}$ 's of the hypermultiplets and the $\sigma_{i}$ 's of the twisted hypermultiplets have actually already appeared in our study of the Fermi multiplet interactions. As seen in Section 2.3, Fermi multiplets defined by $\overline{\mathcal{D}}_{+} \Gamma_{a}=E_{a}\left(\Phi_{i}, \Sigma_{i}\right)$ give a potential $\left|E_{a}\left(\phi_{i}, \sigma_{i}\right)\right|^{2}$, which for our interactive chain of multiplets exhibits quite a few components. From those, the ones that couple $\phi_{i}$ 's and $\sigma_{i}$ 's are the

$$
E_{\Gamma_{i}}\left(\phi_{i}, \sigma_{i}\right)=\sigma_{i} \phi_{i}
$$

or $E_{\tilde{\Gamma}_{i}}=-\tilde{\phi}_{i} \sigma_{i}$, depending on which scalar field we excite inside a certain hypermultiplet. Accordingly, if we choose to excite $\tilde{\sigma}_{i}$ inside a twisted hypermultiplet, instead of its twin $\sigma_{i}$, then these scalars couple through the superpotential term $\left|J_{a}\left(\phi_{i}, \sigma_{i}\right)\right|^{2}$ and, in particular, through the components 


$$
J_{\tilde{\Gamma}_{i}}\left(\phi_{i}, \tilde{\sigma}_{i}\right)=\tilde{\sigma}_{i} \phi_{i}
$$

or $J_{\Gamma_{i}}=\tilde{\phi}_{i} \tilde{\sigma}_{i}$.

These are all the interactions present between the different scalars we choose to excite and which furnish our operator (1.58) with quantum effects. We presume that those are capable of correcting it non-perturbatively to the desired conformal dimension $\Delta_{\mathcal{M}}=\gamma(g)=m-k$.

\subsection{Dual mass}

While the scaling dimension of the meson operator stands as a proposal, there is another insight as to the mass of the BPS state that both enforces the proposed duality and digs out an interesting feature of the field theory.

It is simpler to explore things heuristically here. While coincident branes give massless modes, a superstring suspended between two distanced D2 or D6-branes gives a BPS hypermultiplet (in our kind of theory, presumably of $\mathcal{N}=(4,4)$ supersymmetry) of mass $\sqrt{|\vec{x}|}$, where $\vec{x}$ is the spatial vector connecting the branes. While a hypermultiplet is massless, a mass is obtained by its coupling to a vector superfield, since the latter obtains a VEV through a Fayet-Iliopoulos $D$-term lying on the U(1) gauge theory in the brane worldvolume. That is, as seen from 1.30 and $(1.33)$, for a $\mathrm{U}(1)$ vector superfield we have a $D$-related action

$$
S_{D}=\int \frac{1}{g^{2}} D^{2}+\sigma D \bar{\sigma}-\xi D
$$

where the last term is the Fayet-Iliopoulos term. After integrating out the auxiliary field $D$, the potential energy $V=g^{2}\left(|\sigma|^{2}-\xi\right)^{2}$ is formed which yields the new classical vacuum

$$
\langle\sigma\rangle=\sqrt{\xi},
$$

which in turn couples to the hypermultiplet and is felt as a mass.

When instead we have two stacks, one of $n_{1}$ and another of $n_{2}$ D-branes, we acquire $n_{1} n_{2}$ hypermultiplets that transform under the $\left(n_{1}, \bar{n}_{2}\right)$ representation of $\mathrm{U}\left(n_{1}\right) \times \mathrm{U}\left(n_{2}\right)$. In Hanany-Witten set-ups we have parallel stacks of branes distanced and bordered by NS fivebranes, where the gauge group actually breaks down to $\mathrm{SU}\left(n_{i}\right) \times \mathrm{U}(1)$; the non-trivial $\mathrm{U}(1)$ center provides a Fayet-Iliopoulos $D$-term whose coupling is identified with $\xi=|\vec{x}|$. That is, the $D$-term coupling is given by the distances between the NS fivebranes 68,109 


$$
\xi=\rho_{i+1}-\rho_{i}
$$

Each $\mathrm{U}(1)$ is actually the center of mass of the stack of branes and $D$ is really its Hamiltonian function, where the Fayet-Iliopoulos coupling reflects the fact that we may always add a constant to such a function. While this story is generally studied, let us bring it down onto our case and clarify how it actually works.

By adding a Fayet-Iliopoulos D-term to the $\mathcal{N}=(4,4)$ vector superfield action and integrating out $D$, we acquire the new vacuum $\left\langle\sigma_{i}\right\rangle=\sqrt{\rho_{i+1}-\rho_{i}}=1 / 2$. As restated, $\sigma_{i}$ is one of the scalars of the $\mathcal{N}=(0,4)$ twisted hypermultiplet inside the vector superfield on a stack of D2 or D6-branes, placed between the $(i+1)$ th and $i$ th stack of NS fivebranes. Notice here that we also normalized, by a redefinition, the fundamental $\rho$-interval distance $\rho_{i+1}-\rho_{i}=2 \pi$ to $1 / 4$, for convenience that will become apparent momentarily. Now, this VEV gives a mass to a $\mathcal{N}=(4,4)$ hypermultiplet coupled to it and, in particular for our operator of interest, this is achieved through the interactive terms 1.59 and 1.60 that we brought up in the previous section. That is, if we choose to consider the $\sigma_{i}$ scalar inside the vector superfield and the $\phi_{i}$ scalar inside the hypermultiplet then a mass is acquired by the latter as

$$
\left|E_{\Gamma_{i}}\right|^{2}=\left\langle\sigma_{i}\right\rangle^{2}\left|\phi_{i}\right|^{2}=\frac{1}{4}\left|\phi_{i}\right|^{2}
$$

Accordingly, for other choices of scalar fields inside those multiplets the mass is obtained through other $E$-terms or superpotential $|J|^{2}$ terms with $J$ as in 1.60 .

Now, each such hypermultiplet is actually linked to two stacks of D-branes (gauge nodes), one on its left and one on its right along the $\rho$ dimension. This means that the mass that is gained comes from two VEV contributions, that is

$$
\left|E_{\Gamma_{i}}\right|^{2}+\left|E_{\Gamma_{i+1}}\right|^{2}=\left(\left\langle\sigma_{i}\right\rangle^{2}+\left\langle\sigma_{i+1}\right\rangle^{2}\right)\left|\phi_{i}\right|^{2}=\frac{1}{2}\left|\phi_{i}\right|^{2}
$$

where the mass is now unity. Notice that the value of the mass comes from normalization and thus it is a matter of convention on absolute distances along the $\rho$-dimension. What really matters though is the relative positions of NS fivebranes; changing those shifts the masses of the hypermultiplets in between. Since all the NS fivebranes in our brane set-up are equally separated along $\rho$, accordingly all masses will be the same. Moreover, note that there are as many massive hypermultiplets as the U(1)'s. That is, all hypermultiplets between the gauge nodes along the quiver chain are massive. Therefore we only care about the number of those 
hypermultiplets that contribute to our operator.

Ultimately, the meson operator 1.58 contains $m-k$ scalar fields $\phi_{i}$ which are massive, associating the operator itself with a total classical mass

$$
M_{\mathcal{M}}=m-k
$$

which exactly agrees with the mass 1.53 of the BPS string.

In regard to our particular choice of the BPS operator, besides the agreement on the dual masses it is worth emphasizing the way that this equality is supported. That is, as with the R-charge (or even the presumable anomalous dimension), it again takes both scalar fields $\phi_{i}$ and $\sigma_{i}$ to holographically reflect a dual semiclassical soliton; the $\sigma_{i}$ 's adjust a mass (and a R-charge) and the $\phi_{i}$ 's realize it.

Again, it is the UV particle theory that shapes the proposed meson operator $\mathcal{M}$ and not the actual IR SCFT that sits on the dual side of our $\mathrm{AdS}_{3}$ supergravity backgrounds. While this cautions us to be careful about our statements on what the actual dual BPS operator looks like, we are encouraged by the agreement in mass to make an otherwise bold conjecture: if the choice of operator is correct, then the operator mass somehow transforms into a scaling dimension. This is not as presumptuous as it may sound if we consider that the non-perturbative anomalous dimension $\Delta_{\mathcal{M}}=\gamma(g)=m-k$, that we expect, should be generated by the same interactions that produced the Fayet-Iliopoulos mass. Thus the aforementioned transformation is really thought to be a change on how we realize the same field interactions at different energy scales. That is, the interactions given by 1.59 and 1.60 may be realized as a classical mass in the UV or an anomalous dimension in the IR. This idea is strongly advocated by the fact that the coupling is relevant at the IR of the two-dimensional quantum theory, where the quantum corrections should be important and the scalar masses get integrated out.

As a final comment, the BPS string is a semiclassical bound state which inspires us to assume that its dual operator should too reflect a bound state of two-dimensional fields. That being said, we notice that the operator mass is a sum of all the individual scalar field masses, a fact which renders the UV operator indeed very much alike to a classical bound state of particles. This is a statement on classical bound states in the sense that we neglect an unimportant interaction energy, as we already did with the implicit quantum corrections between fields inside the operator or with the sphere fluctuations on the string mass. While the latter is geometrically obvious through (1.53), the former may be supported by the fact that the gauge coupling is irrelevant at the UV of two-dimensional quantum field theory. 


\subsection{An alternative operator}

Although the last two sections follow the standard examples in the literature (e.g. see (158), there is an alternative choice of bosonic operator dual to the suspended string. Such an operator may be built out of spinor products, which render it bosonic, as long as it satisfies the desired holographic features, i.e. the correct conformal dimension and R-charge.

This can be achieved through products of left and right-handed spinors inside the $\mathcal{N}=$ $(4,4)$ hypermultiplets that connect the two flavor nodes at stake. Ultimately, the operator reads

$$
\mathcal{M}_{k, m}=\bar{\chi}_{+}^{(k)} \cdot \bar{\chi}_{-}^{(k)}\left(\prod_{i=k}^{m-1} \bar{\psi}_{+}^{(i)} \cdot \bar{\lambda}_{-}^{(i)}\right) \bar{\chi}_{+}^{(m)} \cdot \bar{\chi}_{-}^{(m)}
$$

where $\chi_{ \pm}, \psi_{+}$and $\lambda_{-}$are chiral spinors inside the $(4,4)$ hypermultiplets. Again, $\chi_{ \pm}$are spinors inside the end-point hypermultiplets connecting to the flavor nodes. The operator transforms in the bi-fundamental representation of $\mathrm{SU}\left(N_{k}\right) \times \mathrm{SU}\left(N_{m}\right)$ and comprises of mass dimension $\Delta_{\mathcal{M}}^{0}=m-k$ (since $[\psi]=m^{\frac{1}{2}}$ in two dimensions) and $\mathrm{R}[\mathcal{M}]=m-k$, since $\mathrm{R}\left[\psi_{+}\right]=-1$ and $\mathrm{R}\left[\lambda_{-}\right]=0$. Both of those features are exactly what we need.

Though unusual, the new UV operator constitutes a good holographic fit for the suspended string; maybe, it is even better than the more conventional choice of the previous sections, considering that we do not have to assume an IR anomalous dimension or anything else. Nonetheless, there is no obvious reason to choose between the given options of dual operators; as long as the IR SCFT is in the shadows, both of them could be correct. In fact, we could also build operators that are combinations of those two, which would also fit the desired standards. As a final remark, note that even if the scaling dimension of the operator 1.67) exhibits small corrections in the IR, this holographically agrees with the small mass corrections of the BPS string due to its $\mathrm{S}^{2}$-fluctuations that we neglected in 1.53 .

\section{Conclusions}

Summarizing, in Section 3 we studied all possible categories of vacua within a particular $\mathrm{AdS}_{3}$ family of massive IIA supergravity solutions, first given in [34]. Apart from the original solutions introduced there, we presented the remaining types of vacua in the same family which all naively seem to give anomalous dual quiver gauge theories. We proved that these erratic solutions imply D-branes on the boundary of the space, which in turn correspond to flavor symmetries that exactly cancel the apparent gauge anomalies. A special feature of the situation is that, due to strong RR fluxes on the boundary of the space, these D-branes 
come exclusively in bound states forming polarizations that provide the quiver with flavor in a quite idiosyncratic way.

After dealing with all possible kinds of solutions and quiver theories, in Section 4 we supplement the quiver structure with additional matter in the form of bifundamental links between color and flavor nodes. These, we argue, may only be $\mathcal{N}=(4,4)$ hypermultiplets corresponding to suspended superstrings between D2 and D4-branes or D6 and D8-branes in the ancestral Hanany-Witten set-up.

Having introduced the complementary bifundamental matter too, in Section 5 we put holography to the test by considering a semiclassical string inside the $\mathrm{AdS}_{3}$ background stretched between two D-branes. We call this a meson string and by finding its mass and R-charge we show it is a BPS state. Next, we propose a UV operator out of fundamental scalar fields, which we argue it may be dual to the BPS soliton. Moreover, crucial to the construction of this operator is the additional bifundamental matter we have introduced. While the R-charge of the proposed operator seems to get along with our expectations, its conformal dimension is classically zero since scalar fields in two spacetime dimensions have a vanishing mass dimension. What is more, since the two-dimensional SCFT we are assuming is strongly coupled and these $\mathrm{AdS}_{3}$ vacua have been proven to be non-integrable, the perturbative regime of calculations is out of our reach. Nonetheless, by bringing to the surface the superpotential of the UV quiver theory, we find interactions between the scalars inside the operator and we are led to the conclusion that the latter could be acquiring a totally non-perturbative anomalous dimension at the IR, equal to the mass of the BPS string.

Pursuing the holographic picture of the meson string, we focus on the quiver structure and find that scalars inside the vector superfields should obtain a VEV through a Fayet-Iliopoulos term. The latter is due to the $\mathrm{U}(1)$ theory inside the $\mathrm{U}(N)$ gauge group of each stack of branes in the set-up. Superpotential interactions between the vector and hypermultiplets then dictate that bifundamental matter acquires a mass, ultimately associating the dual meson operator with a classical mass equal to that of the BPS string. Since the operator mass is a sum of all the individual scalar field masses, this renders the operator indeed very much alike to a classical bound state of particles dual to a bound string state between D-branes.

Finally, we propose an alternative operator dual to the BPS soliton. This new object is made out of spinor products, which ultimately renders it a bosonic quantity. A simple calculation shows that this operator has the correct scaling dimension and R-charge, which makes it a good holographic fit for the dual BPS string, but, nonetheless, there is no a priori reason to choose this operator over the other or, even, over a combination of both. 


\section{G Extremal $p$-brane solutions}

Extremal $p$-branes are supergravity solutions that in the context of superstring theory are identified with stacks of D $p$-branes. These are distinct from O-planes that essentially constitute boundary conditions for strings. The leading order backgrounds for all the above read

$$
\begin{array}{llll}
p \text {-brane } & : \quad \mathrm{d} s^{2} \sim x^{\frac{7-p}{2}} \mathrm{~d} s_{\mathrm{M}^{1, p}}^{2}+x^{\frac{p-7}{2}}\left(\mathrm{~d} x^{2}+x^{2} \mathrm{~d} s_{\Sigma^{8-p}}^{2}\right), & e^{\phi} \sim x^{\frac{(3-p)(p-7)}{4},} \\
p \text {-brane } \tilde{\Sigma}^{s}: & \mathrm{d} s^{2} \sim x^{\frac{7-p-s}{2}} \mathrm{~d} s_{\mathrm{M}^{1, p}}^{2}+x^{\frac{p+s-7}{2}}\left(\mathrm{~d} x^{2}+\mathrm{d} s_{\tilde{\Sigma}^{s}}^{2}+x^{2} \mathrm{~d} s_{\Sigma^{8-p-s}}^{2}\right), & e^{\phi} \sim x^{\frac{(3-p)(p+s-7)}{4},}, \\
\text { Op-plane } \quad: & \mathrm{d} s^{2} \sim \frac{1}{\sqrt{x}} \mathrm{~d} s_{\mathrm{M}^{1, p}}^{2}+\sqrt{x}\left(\mathrm{~d} x^{2}+\mathrm{d} s_{\Sigma^{8-p}}^{2}\right), & e^{\phi} \sim x^{\frac{3-p}{4},}
\end{array}
$$

where we schematically acknowledge constants. Here $\mathrm{M}^{1, p}$ is a manifold that the brane fills, $\Sigma^{8-p}$ is a compact space - on which one integrates to obtain the associated charge of the brane - and $\tilde{\Sigma}^{s}$ is the manifold over which a brane may be smeared.

\section{H Two dimensional $\mathcal{N}=(0,4)$ superfields}

\section{H.1 Field content and action}

Traditionally, extended supersymmetric theories are best realized through constituent, minimal supersymmetric multiplets. $\mathcal{N}=(0,4)$ supersymmetry is no different and boils down to $\mathcal{N}=(0,2)$ superfields, which we now introduce. The language and content we present is mainly based on [155, 156], which both hold excellent reviews on the subject.

Gauge multiplet This is a real superfield, $\mathcal{V}$, which comprises of an adjoint-valued complex left-handed fermion $\zeta_{-}$, a real auxiliary field $D$ and a gauge field $A$. The standard kinetic term for the gauge multiplet expands into the action

$$
S_{\text {gauge }}=\frac{1}{g^{2}} \operatorname{Tr} \int \mathrm{d}^{2} x\left(\frac{1}{2} F_{01}^{2}+i \bar{\zeta}_{-}\left(\mathcal{D}_{0}+\mathcal{D}_{1}\right) \zeta_{-}+D^{2}\right)
$$

Chiral multiplet A $\mathcal{N}=(0,2)$ chiral superfield, $\Phi$, comprises of a right-moving fermion $\psi_{+}$and a complex scalar $\phi$, which both transform in the same gauge group representation. The kinetic term for the gauged chiral multiplet expands into 


$$
S_{\text {chiral }}=\int \mathrm{d}^{2} x\left(-\left|D_{\mu} \phi\right|^{2}+i \bar{\psi}_{+}\left(D_{0}-D_{1}\right) \psi_{+}-i \bar{\phi} \zeta_{-} \psi_{+}+i \bar{\psi}_{+} \bar{\zeta}_{-} \phi+\bar{\phi} D \phi\right)
$$

Fermi multiplet This is an anticommuting superfield, $\Psi$, containing a left-moving spinor $\psi_{-}$and a complex auxiliary field $G$. The Fermi superfield is constrained by $\overline{\mathcal{D}}_{+} \Psi=E$ where $\mathcal{D}_{+}=\partial_{\theta^{+}}-i \bar{\theta}^{+}\left(\mathcal{D}_{0}+\mathcal{D}_{1}\right)$, with $\mathcal{D}_{0,1}=\partial_{0,1}+i A_{0,1}$ and $E=E\left(\Phi_{i}\right)$ a holomorphic function of the chiral superfields $\Phi_{i}$. The kinetic term for the Fermi multiplet expands into

$$
S_{\mathrm{Fermi}}=\int \mathrm{d}^{2} x\left(i \bar{\psi}_{-}\left(D_{0}+D_{1}\right) \psi_{-}+|G|^{2}-\left|E\left(\phi_{i}\right)\right|^{2}-\bar{\psi}_{-} \frac{\partial E}{\partial \phi_{i}} \psi_{+i}+\bar{\psi}_{+i} \frac{\partial \bar{E}}{\partial \bar{\phi}_{i}} \psi_{-}\right)
$$

The holomorphic function $E\left(\phi_{i}\right)$ comes up as a potential $\sim\left|E\left(\phi_{i}\right)\right|^{2}$ inside the action and thus its particular choice, along with superpotential terms, determine the interactions of the theory.

Superpotentials Considering multiple Fermi superfields $\Psi_{a}$ which couple to scalar chiral superfields $J^{a}\left(\Phi_{i}\right)$ through $S_{J} \sim \int \Psi_{a} J^{a}$ over half of the superspace, supersymmetry dictates that superfields are constrained as $E \cdot J=\sum_{a} E_{a} J^{a}=0 . \quad J^{a}(\phi)$ produce potential terms $\sim\left|J^{a}\left(\phi_{i}\right)\right|^{2}$ which are usually referred to as the superpotential in $\mathcal{N}=(0,2)$ theories. Therefore, besides the $E$-terms, the $J$-terms also give potential terms in $\mathcal{N}=(0,2)$ supersymmetric theories, all of them directly connected to Fermi multiplets. The attachment $E \cdot J=0$ when multiple Fermi and chiral multiplets are present, decides for the particular interactions in the theory. But to see how this plays out we must first introduce $\mathcal{N}=(0,4)$ supersymmetric multiplets.

Two dimensional $\mathcal{N}=(0,4)$ supersymmetry has four real right-moving supercharges that rotate in the $(\mathbf{2}, \mathbf{2})_{+}$representation of a $\mathrm{SO}(4)_{R} \cong \mathrm{SU}(2)_{R} \times \mathrm{SU}(2)_{R}$ R-symmetry, where the plus sign indicates the chirality under the $\mathrm{SO}(1,1)$ Lorentz group. The superfields in this kind of theories are the following.

$\mathcal{N}=(0,4)$ vector multiplet Since in two dimensions the gauge field is not propagating it is natural that two-dimensional $\mathcal{N}=(0,4)$ vector superfields are composed of left-handed spinors, which don't transform under right-moving supersymmetry. Thus, a $\mathcal{N}=(0,4)$ vector superfield consists of an adjoint-valued $\mathcal{N}=(0,2)$ Fermi superfield $\Theta$ and a $\mathcal{N}=(0,2)$ vector superfield . 
Besides the gauge field, there are two left-handed complex fermions, $\zeta_{-}^{a}$ and three auxiliary fields, transforming in the $(\mathbf{2}, \mathbf{2})_{-}$and $(\mathbf{3}, \mathbf{1})$ R-symmetry representations, respectively. The Fermi superfield is constrained through $\overline{\mathcal{D}}_{+} \Theta=E_{\Theta}$ with $E_{\Theta}$ depending on the matter content, i.e. the chiral superfields present in the theory.

$\mathcal{N}=(0,4)$ hypermultiplet The first way to couple matter fields to a $\mathcal{N}=(0,4)$ vector multiplet (essentially to its constituent $\mathcal{N}=(0,2)$ Fermi multiplet) is to consider a $\mathcal{N}=(0,4)$ hypermultiplet that consists of two $\mathcal{N}=(0,2)$ chiral superfields, $\Phi$ and $\tilde{\Phi}$, which transform in conjugate gauge group representations and whose pairs of complex scalars and right-handed spinors transform in the $(\mathbf{2}, \mathbf{1})$ and $(\mathbf{1}, \mathbf{2})+$ representations, respectively, under the R-symmetry.

$\mathcal{N}=(0,4)$ twisted hypermultiplet Another possible way to couple matter fields to a $\mathcal{N}=(0,4)$ vector multiplet $\mathcal{N}=(0,4)$ is through a twisted hypermultiplet. This consists of a pair of $\mathcal{N}=(0,2)$ chiral multiplets, $\Sigma$ and $\tilde{\Sigma}$, which too transform in conjugate gauge group representations. Now, nonetheless, different R-charge is being enforced by the coupling to the Fermi field $\Theta$. In contrast to hypermultiplets, the scalars and right-handed spinors now transform in the $(\mathbf{1}, \mathbf{2})$ and $(\mathbf{2}, \mathbf{1})_{+}$representations of R-symmetry.

$\mathcal{N}=(0,4)$ Fermi multiplet Those contain two $\mathcal{N}=(0,2)$ Fermi superfields, $\Gamma$ and $\tilde{\Gamma}$, which transform in conjugate gauge group representations and whose left-moving spinors transform in the $(\mathbf{1}, \mathbf{1})_{-}$R-symmetry representation.

$\mathcal{N}=(0,2)$ Fermi multiplet Finally, it is acceptable in $\mathcal{N}=(0,4)$ supersymmetric theories to consider $\mathcal{N}=(0,2)$ Fermi multiplets, as long as their left-moving spinors are $\mathrm{SO}(4)_{R}$ singlets and, according to that R-symmetry transformation, couple appropriately to the rest of the matter in the theory.

As we are about to see, our quantum field theory also contains $\mathcal{N}=(4,4)$ superfields that decompose under $\mathcal{N}=(0,4)$ supersymmetry into their $\mathcal{N}=(0,4)$ superfield constituents. The $\mathcal{N}=(4,4)$ vector multiplet splits into an $\mathcal{N}=(0,4)$ vector multiplet and an adjointvalued $\mathcal{N}=(0,4)$ twisted hypermultiplet. The chiral superfields $\Sigma$ and $\tilde{\Sigma}$ inside the twisted hypermultiplet couple to the Fermi multiplet $\Theta$ inside the $\mathcal{N}=(0,4)$ vector superfield. Finally, a $\mathcal{N}=(4,4)$ hypermultiplet decomposes into an $\mathcal{N}=(0,4)$ hypermultiplet, $\Phi$ and $\tilde{\Phi}$, 
and an $\mathcal{N}=(0,4)$ Fermi multiplet, $\Gamma$ and $\tilde{\Gamma}$.

\section{H.2 U(1) R-charge}

From the $\mathrm{SU}(2)_{R} \times \mathrm{SU}(2)_{R}$ R-symmetry of the $\mathcal{N}=(0,4)$ theory, we single out a $\mathrm{U}(1)_{R}$ inside one $\mathrm{SU}(2)_{R}$ and give the $\mathrm{U}(1)_{R}$ charge of each fermion in the above multiplets.

For the $\mathcal{N}=(0,4)$ vector multiplet we have that the left-handed fermion inside the vector has $R\left[\zeta_{-}\right]=+1$ while the same holds for the left-handed fermion inside the Fermi multiplet, i.e. $\mathrm{R}\left[\psi_{-}\right]=+1$. On the contrary, both right-handed fermions inside the $\mathcal{N}=(0,4)$ twisted hypermultiplet have $\mathrm{R}\left[\psi_{+}\right]=0$. For both right-handed fermions inside the $\mathcal{N}=(0,4)$ hypermultiplet we have $\mathrm{R}\left[\psi_{+}\right]=-1$. Finally, the fermion inside the $\mathcal{N}=(0,2)$ Fermi multiplet is uncharged under R-symmetry.

\section{The D8/D4 bound state}

We consider the background of the case with a constant $u$ function and study the beginning of its $\rho$-dimension where D4-branes seem to polarize into a D8/D4 bound state. The fact

that $C_{9}^{e l}$ field becomes infinitely strong at that endpoint reasonably makes the D8/D4 bound state dominant, yet a more formal proof of it being the true vacuum is in order.

Comparing to Myers's original calculation [157], here we are dealing with higher dimensional branes. Furthemore, the method developed in [157 holds in the flat space limit, whereas our bound state takes place in $\mathrm{AdS}_{3} \times \mathrm{S}^{2} \times \mathrm{CY}_{2} \times \mathrm{I}_{\rho}$. What is more, Calabi-Yau manifolds lack a particular metric tensor whatsoever.

However, the situation is less dramatic than it may look. First of, the Chern-Simons term

$$
S_{\mathrm{CS}}^{\mathrm{D} 4}=\mu_{4} \int \operatorname{Tr} \sum e^{i \lambda \imath_{\Phi} \imath_{\Phi}} C_{(n)} e^{\mathcal{F}_{2}}
$$

gets only deformed away from the flat space limit by terms coupled to the $B_{2}$ field. These terms would be unimportant compared to our infinitely strong $C_{9}^{\text {el }}$ potential coupling, but the Kalb-Ramond field vanishes at $\rho=0$ for constant $u(\rho)$ anyway. Next, the Dirac-Born-Infeld (DBI) action

$$
S_{\mathrm{DBI}}^{\mathrm{D} 4}=-T_{4} \int \mathrm{d}^{4} \xi \operatorname{Tr} e^{-\phi} \sqrt{-\operatorname{det}\left(G_{a b}+G_{a i}\left(Q^{-1}-\delta\right)^{i j} G_{j b}+\lambda \tilde{f}_{a b}\right) \operatorname{det}\left(Q_{j}^{i}\right)},
$$


where

$$
Q_{j}^{i}=\delta_{j}^{i}+i \lambda\left[\Phi^{i}, \Phi^{k}\right] G_{k j}
$$

The $a, b$ are indices pulled-back on the D4-brane worldvolumes, while $i, j$ are their transverse dimensions. That is, $G_{\mu \nu}=\left(G_{a b}, G_{i j}\right)$ where $G_{a i}=0$ and the transverse field $G_{i j}$ includes the $\rho$-dimension and an independent $\mathrm{CY}_{2}$ block.

Choosing a static gauge where the D4-branes' worldvolumes fill up $\mathrm{AdS}_{3} \times \mathrm{S}^{2}$, i.e. choosing worldvolume coordinates and the transverse modes (which are scalars in the D4 worldvolume) as

$$
\xi^{a}=X^{a}=(t, x, r, \theta, \phi), \quad X^{i}\left(\xi^{a}\right)=\lambda \Phi^{i}\left(\xi^{a}\right),
$$

where the $\lambda$ was included on dimensional grounds, then the DBI action reads

$$
S_{\mathrm{DBI}}^{\mathrm{D} 4}=-T_{4} \int \mathrm{d}^{4} \xi \operatorname{Tr} e^{-\phi} \sqrt{-\operatorname{det}\left(G_{a b}+\lambda^{2} \partial_{a} \Phi^{i} \partial_{b} \Phi^{j} G_{i j}\right) \operatorname{det}\left(\delta_{j}^{i}+i \lambda\left[\Phi^{i}, \Phi^{k}\right] G_{k j}\right)},
$$

where we ignored the D4-brane gauge field $\tilde{f}$ as unimportant. Using the fact that the determinant behaves like $\operatorname{det}(A+\lambda B)=\operatorname{det} A+\lambda \operatorname{Tr} B+\ldots$ for small $\lambda$, we obtain the potential energy

$$
V(\Phi)=N_{4} T_{4} M_{4}-\frac{T_{4} M_{4} \lambda^{2}}{4} \operatorname{Tr}\left[\Phi^{i}, \Phi^{j}\right]^{2}-i \frac{T_{4} M_{4} \lambda^{3}}{12} \operatorname{Tr}\left[\Phi^{i}, \Phi^{j}\right]^{3}+\ldots
$$

where the ellipsis contains higher-order potential terms and contractions with the transverse metric $G_{i j}$ are implied. $N_{4}$ is the number of D4-branes and $M_{4}$ comes from the factor $e^{-\phi} \operatorname{det} G$, which for our background 1.40 at $\rho \rightarrow 0$ scales as

$$
e^{-\phi} \operatorname{det} G \stackrel{\rho \rightarrow 0}{\longrightarrow} M_{4} \rho^{\frac{5}{4}} \rho^{-\frac{5}{4}}=M_{4}
$$

which goes to a constant. Notice that in the flat space limit, the second term of 1.77 reflects the familiar SYM potential.

So far, the sole deviation from the flat space analysis is the contraction of indices in the potential (1.77) with the transverse metric $G_{i j}$. This field includes the $\rho$-dimension 
component and an independent $\mathrm{CY}_{2}$ block. The former is known but unimportant since the $\Phi^{\rho}$ modes will not be ultimately involved in the potential energy and thus no such indices will need to contract, while the latter is essential but lacks a particular metric tensor. We could maybe realize some generic algebraic constraints on the Calabi-Yau block, like its Ricci flatness, but we do need a particular metric tensor which makes it is easier to assume $\mathrm{CY}_{2}=$ $\mathrm{T}^{4}$ and thus let for a Euclidean $\mathbb{R}^{4}$ metric.

Our study significantly simplifies by choosing a convenient gauge for the RR potential as

$$
C_{9}^{e l}=-\frac{u^{2}}{h_{8}} \operatorname{vol}\left(\mathrm{AdS}_{3}\right) \wedge \operatorname{vol}\left(\mathrm{S}^{2}\right) \wedge \operatorname{vol}\left(\mathrm{CY}_{2}\right)
$$

On these grounds, while picking the static gauge 1.75 , we can expand the source term

$$
\begin{aligned}
S_{\mathrm{CS}}^{\mathrm{D} 8 / \mathrm{D} 4}=-\frac{\lambda^{2}}{2} \mu_{8} \int \operatorname{Tr}\left(\imath_{\Phi} \imath_{\Phi}\right)^{2} C_{9}^{e l} & =-\frac{\lambda^{2}}{2} \mu_{4} \int \mathrm{d}^{5} \xi \operatorname{Tr} \Phi^{i} \Phi^{j} \Phi^{k} \Phi^{l} C_{i j k l t x r} \phi \phi \\
& =-\frac{\lambda^{2}}{8} \mu_{4} \int \mathrm{d}^{5} \xi \operatorname{Tr}\left[\Phi^{i}, \Phi^{j}\right]\left[\Phi^{k}, \Phi^{l}\right] C_{9},
\end{aligned}
$$

where we redefine the Latin letters $i, j, k, l$ to denote only $\mathrm{CY}_{2}$ directions. The transverse modes $\Phi^{i}$ are in general anticommuting matrices, where the diagonal elements are the positions of the D4-branes, while the non-diagonal ones reflect their quantum geometry due to the superposition of strings ending on them. The fact that $\Phi^{i}$ are oscillations in non-flat dimensions is not restrictive in any way, since we fundamentally assume those modes as generic anticommuting matrices that may (and actually do) give a fuzzy geometry. Also, note that in general we should include $\Phi^{\rho}$ too, but not in our particular gauge of $C_{9}^{e l}$.

Now we want to focus on $\rho=0$ where all the action takes place, i.e. expand $C_{9}^{e l}$ around that endpoint. It being a singular endpoint implies a Laurent expansion but, since it is also the endpoint of a closed interval, this series is not well defined around it. Thus, we just pick a point $x$ close to $\rho=0$ and expand around it, inside a circular region (of the complex domain) - of radius $x$ too - which touches the singularity. That is, the expansion reduces to a Taylor series around $x$ as

$$
S_{\mathrm{CS}}^{\mathrm{D} 8 / \mathrm{D} 4}=-\frac{\lambda^{2}}{8} \mu_{4} \int \mathrm{d}^{5} \xi \operatorname{Tr}\left[\Phi^{i}, \Phi^{j}\right]\left[\Phi^{k}, \Phi^{l}\right]\left(\left.C_{9}\right|_{\rho=x}+\left.\lambda \Phi^{\rho} F_{10}\right|_{\rho=x}+\ldots\right)
$$

Since $h_{8} \rightarrow 0$ for small $x$, the RR fields $C_{9}$ and $F_{10}$ blow up there and thus from now on we will consider them as largely-valued quantities.

The above source term adds to the interactions (1.77) of the DBI action and hence, taking 
into account the full D4-brane action $S=S_{\mathrm{DBI}}+S_{\mathrm{CS}}$, we acquire the potential energy

$$
\begin{aligned}
V(\Phi)= & -\frac{\lambda^{2}}{4} \operatorname{Tr}\left[\Phi^{i}, \Phi^{j}\right]^{2}+\left.\frac{\lambda^{2}}{8} \operatorname{Tr}\left[\Phi^{i}, \Phi^{j}\right]\left[\Phi^{k}, \Phi^{l}\right] C_{9}\right|_{\rho=x} \\
& -i \frac{\lambda^{3}}{12} \operatorname{Tr}\left[\Phi^{i}, \Phi^{j}\right]^{3}+\left.\frac{\lambda^{3}}{8} \operatorname{Tr}\left[\Phi^{i}, \Phi^{j}\right]\left[\Phi^{k}, \Phi^{l}\right] \Phi^{\rho} F_{10}\right|_{\rho=x}
\end{aligned}
$$

where we have assumed a constant mode $\Phi^{\rho}$ to simplify the game and reparametrized the fields conveniently to absorb numerical factors. Reparametrizing once more, the potential gets an order by order variation $\frac{\partial V}{\partial \Phi}=0$ as

$$
\begin{array}{ll}
\mathcal{O}\left(\lambda^{2}\right): & {\left[\Phi^{i}, \Phi^{j}\right]=\left[\Phi^{k}, \Phi^{l}\right] C_{i j k l \ldots},} \\
\mathcal{O}\left(\lambda^{3}\right): & {\left[\Phi^{i}, \Phi^{j}\right]\left[\Phi^{j}, \Phi^{k}\right]=-i\left[\Phi^{l}, \Phi^{m}\right] F_{i k l m \ldots}}
\end{array}
$$

which has a trivial solution $\left[\Phi^{i}, \Phi^{j}\right]=0$ giving $V_{0}=0$, corresponding to separated D4-branes. Alternatively, combining both of these equations, the potential also exhibits the non-trivial solution

$$
\left[\Phi^{i}, \Phi^{j}\right]=-i \epsilon^{i j} \partial_{\rho}
$$

which in momentum space reads

$$
\left[\Phi^{i}, \Phi^{j}\right]=\epsilon^{i j} p_{\rho}
$$

where we abuse the antisymmetric tensor just to sustain the antisymmetry of the commutator into the rhs. Placing this solution back into the SYM potential we get

$$
\left.V_{\star} \cong \lambda^{2} p_{\rho}^{2} C_{9}\right|_{\rho \rightarrow 0}+\mathcal{O}\left(\lambda^{3}\right)
$$

where we used the fact that $C_{9}$ is large at $\rho \rightarrow 0$.

As a matter of fact, $C_{9}$ is not only large but also negative at that endpoint, which means that $V_{\star}<0$. Since the separated D4-branes correspond to the null energy state $V_{0}=0$, the latter is unstable and condenses out into the non-trivial D8/D4 bound state with $V_{\star}$ which is the true stable vacuum at $\rho=0$. Also, notice the fact that specifically $V_{\star} \rightarrow-\infty$, due to the strong RR potential $C_{9} \rightarrow-\infty$ at $\rho \rightarrow 0$, which saves us from having to also investigate 
other bound states. In our case, $C_{3}^{e l}, C_{7}^{e l} \rightarrow 0$ at $\rho \rightarrow 0$ anyway, but even if this was not the case there just cannot be any lower energy than $V_{\star}$.

\section{J R-charge of the BPS state}

Naively, the $B_{2}$ field in (1.1) has nothing to do with the 1-form $\cos \theta \mathrm{d} \phi$. However, $B_{2}$ exhibits large gauge transformations across the $\rho$-intervals $[2 \pi k, 2 \pi(k+1)]$, which are explicitly realized through the 1 -form

$$
\Lambda_{1}=\Theta(\rho-2 \pi k) \Theta(2 \pi(k+1)-\rho) \pi k \cos \theta \mathrm{d} \phi
$$

Therefore, the large gauge transformations $B_{2} \rightarrow B_{2}+\mathrm{d} \Lambda_{1}$ read

$$
\begin{aligned}
B_{2} \longrightarrow B_{2}+\Theta(\rho-2 \pi k) & \Theta(2 \pi(k+1)-\rho) \pi k \mathrm{~d} \Omega_{2} \\
& +[\delta(\rho-2 \pi k)-\delta(2 \pi(k+1)-\rho)] \pi k \mathrm{~d} \rho \wedge \cos \theta \mathrm{d} \phi
\end{aligned}
$$

where, in this explicit formulation, the only difference now is the novel delta-terms, $B_{2}^{\delta}$. The latter, which are the ones producing the R-charge, are integrated over a $\rho$-interval as

$$
\begin{aligned}
\frac{1}{2 \pi} \int B_{2}^{\delta}=\frac{2}{2 \pi} \int_{\mathbb{R}} \cos \theta \mathrm{d} \phi \int_{2 \pi k}^{2 \pi(k+1)} \mathrm{d} \rho\{ & {[\delta(\rho-2 \pi k)-\delta(2 \pi(k+1)-\rho)] \pi k } \\
& -\delta(2 \pi k-\rho) \pi(k-1)+\delta(\rho-2 \pi(k+1)) \pi(k+1)\},
\end{aligned}
$$

where the first line is the contribution coming from $B_{2}^{\delta}$ defined on the interval $[2 \pi k, 2 \pi(k+1)]$ as expected, while the second line includes the contributions coming from the intervals prior and next to that. Considering $\int_{0}^{\infty} \delta(x) \mathrm{d} x=1 / 2$, the above integral gives

$$
\frac{1}{2 \pi} \int B_{2}^{\delta}=\int_{\mathbb{R}} \cos \theta \mathrm{d} \phi
$$

and the whole meson string $\mathcal{M}_{k, m}$ acquires the R-charge source term

$$
S_{\mathcal{M}}=(m-k) \int_{\mathbb{R}} \cos \theta \mathrm{d} \phi
$$


which yields its R-charge

$$
Q_{R}=m-k .
$$





\section{Part III}

\section{Appendices and Bibliography}





\section{Chapter 1}

\section{Supersymmetry in two dimensions}

In this appendix we briefly review supersymmetry and its realization in two spacetime dimensions. After summarizing the basics of superspace and superfields, we dive into twodimensional superfields and, in particular, we introduce $\mathcal{N}=(0,2)$ supersymmetric multiplets. From the latter we build up $\mathcal{N}=(0,4)$ and $\mathcal{N}=(4,4)$ superfields in two dimensions, while we show the way those decompose in a theory of smaller supersymmetry. Finally, we point out the different realizations of R-symmetry while we dimensionally reduce a supersymmetric theory. Standard references on these subjects are $154,156,159,160$.

\section{A Superspace and superfields}

In usual spacetime, the symmetry generators of a Lie algebra produce appropriate transformations on spacetime coordinates and leave the theory intact. The simplest example is translation invariance, that is invariance under the transformation

$$
x^{\prime \mu}=x^{\mu}+a^{\mu},
$$

where $a^{\mu}$ parametrize the translations. In particular, they parametrize the (differential) translation generators on a group element, $U=\exp \left(i a^{\mu} P_{\mu}\right)$, which acts on spacetime fields and coordinates.

Supersymmetry, on the other hand, is generated by anticommuting (Grassmann) generators $Q_{\alpha}, \bar{Q}_{\dot{\alpha}}$ which are parametrized analogously by spinors $\epsilon_{\alpha}, \bar{\epsilon}_{\dot{\alpha}}$, building up transformations $U=\exp (i \epsilon \cdot Q+i \bar{\epsilon} \cdot \bar{Q})$. Therefore, if we want to realize supersymmetry charges as producing a translation too on the fields, we may treat $\epsilon, \bar{\epsilon}$ as displacement vectors in an extended spacetime with extra Grassmann coordinates. We choose the supersymmetry charges to be minimal spinors and so the same follows for $\epsilon, \bar{\epsilon}$ that parametrize them. Thus, the superspace that we are looking for has extra Grassmann coordinates $\theta^{\alpha}, \bar{\theta}^{\dot{\alpha}}$ in addition to the usual spacetime coordinates $x^{\mu}$. 
A superfield is a field with dependence on the whole superspace as $\mathcal{S}(x, \theta, \bar{\theta})$. Since, in a theory of states, a (super) field transforms unitarily as

$$
\left\langle s_{1}\left|\mathcal{S}\left(x^{\prime}, \theta^{\prime}, \bar{\theta}^{\prime}\right)\right| s_{2}\right\rangle=\left\langle s_{1}\left|U(a, \epsilon, \bar{\epsilon}) \mathcal{S}(x, \theta, \bar{\theta}) U^{\dagger}(a, \epsilon, \bar{\epsilon})\right| s_{2}\right\rangle
$$

then, using the superalgebra, we find that a translation in superspace reads

$$
\begin{aligned}
x^{\prime \mu} & =x^{\mu}+a^{\mu}+i \epsilon \sigma^{\mu} \bar{\theta}-i \theta \sigma^{\mu} \bar{\epsilon}, \\
\theta^{\prime} & =\theta+\epsilon, \\
\bar{\theta}^{\prime} & =\bar{\theta}+\bar{\epsilon} .
\end{aligned}
$$

Through a general supersymmetric transformation $U=\exp \left(i a^{\mu} P_{\mu}+i \epsilon \cdot Q+i \bar{\epsilon} \cdot \bar{Q}\right)$, this reveals the differential form of the supercharges,

$$
\begin{aligned}
Q_{\alpha} & =\frac{\partial}{\partial \theta^{\alpha}}-i \sigma_{\alpha \dot{\alpha}}^{\mu} \bar{\theta}^{\dot{\alpha}} \frac{\partial}{\partial x^{\mu}} \\
\bar{Q}_{\dot{\alpha}} & =-\frac{\partial}{\partial \bar{\theta}^{\dot{\alpha}}}+i \sigma_{\alpha \dot{\alpha}}^{\mu} \theta^{\alpha} \frac{\partial}{\partial x^{\mu}}
\end{aligned}
$$

where $\alpha, \dot{\alpha}$ are the two chiralities of spinor indices.

The simplest kind of superfield that may live in superspace is obviously a scalar (commuting) superfield $\Phi(x, \theta, \bar{\theta})$. However this field has a large field content, since it expands as

$$
\Phi(x, \theta, \bar{\theta})=\phi(x)+\theta \cdot \psi(x)+\bar{\theta} \cdot \chi(x)+\theta \cdot \theta A(x)+\ldots
$$

up to nine terms. If we want to use superfields to build the simplest kind of theories (i.e. the Wess-Zumino kind of Lagrangians of a scalar with a spinor superpartner and an auxiliary field) we must somehow constrain $\Phi(x, \theta, \bar{\theta})$ to a minimal field content. Observing (1.5), we notice that if $\Phi$ is a function of $x$ and $\theta$ only, then it becomes substantially minimal. Thus, we can naively demand $\Phi$ to be independent of $\theta$ or $\bar{\theta}$, that is

$$
\frac{\partial}{\partial \bar{\theta}^{\dot{\alpha}}} \Phi=0
$$

However, this is not consistent with supersymmetry since, considering (1.5), even if we assume $\Phi=\Phi(x, \theta)$ then a supersymmetric transformation shall spread it back over all 
superspace. It turns out that, if we demand a general constraint

$$
\bar{D} \mathcal{S}(x, \theta, \bar{\theta})=0
$$

then the operation $D$ has to obey $\{D, Q\}=\{D, \bar{Q}\}=0$ for supersymmetry to be preserved. This leads to the operators

$$
\begin{aligned}
& D_{\alpha}=\frac{\partial}{\partial \theta^{\alpha}}+i \sigma_{\alpha \dot{\alpha}}^{\mu} \bar{\theta}^{\dot{\alpha}} \frac{\partial}{\partial x^{\mu}}, \\
& \bar{D}_{\dot{\alpha}}=-\frac{\partial}{\partial \bar{\theta}^{\dot{\alpha}}}-i \sigma_{\alpha \dot{\alpha}}^{\mu} \theta^{\alpha} \frac{\partial}{\partial x^{\mu}} .
\end{aligned}
$$

Therefore, the simplest superfield we can realize is a (left-) chiral superfield which obeys $\bar{D}_{\dot{\alpha}} \Phi=0$ and expands as

$$
\Phi(x, \theta, \bar{\theta})=\phi(x)+\theta \cdot \psi(x)+\theta \cdot \theta F(x)+\ldots,
$$

where the dots indicate derivative terms of the above fields, of higher order in $\theta, \bar{\theta}$. Observing that $\bar{D}_{\dot{\alpha}}$ leaves the coordinate $y^{\mu}=x^{\mu}+i \theta \sigma^{\mu} \bar{\theta}$ invariant, i.e. $\bar{D}_{\dot{\alpha}} y^{\mu}=0$, then the left-chiral superfield may be written in the compact form

$$
\Phi(y, \theta)=\phi(y)+\theta \cdot \psi(y)+\theta \cdot \theta F(y)
$$

while its complex conjugate $\bar{\Phi}$ is an antichiral superfield obeying $D_{\alpha} \bar{\Phi}=0$.

\section{A.1 Supersymmetric action terms}

Taking the supersymmetric variation of a chiral superfield

$$
\Phi\left(x^{\prime}, \theta^{\prime}, \bar{\theta}^{\prime}\right)=\Phi(x, \theta, \bar{\theta})+i\left(\epsilon \sigma^{\mu} \bar{\theta}-\theta \sigma^{\mu} \bar{\epsilon}\right) \partial_{\mu} \Phi+\epsilon \cdot \partial \Phi+\bar{\epsilon} \cdot \bar{\partial} \Phi+\ldots
$$

and expanding in component fields, we find that the auxiliary field transforms as a total derivative, i.e. $\delta F \sim \bar{\epsilon} \cdot \not \partial \psi$. Stated otherwise, the coefficient of the $\theta \cdot \theta$ term in the expansion of a left-chiral superfield $\Phi(x, \theta, \bar{\theta})$ transforms as a total derivative under supersymmetry. These we call the $F$-terms of chiral superfields and in the simple case of using just one left-chiral superfield $\Phi$ we can construct a supersymmetry-invariant action term as $\int \mathrm{d}^{2} \theta \Phi=\left.\Phi\right|_{F}=F$. 
Of course, there is no interest in a term containing just an auxiliary field, which means we desire more complicated left-chiral superfields accompanied by more interesting $F$-terms. The key observation is that the product of any number of left-chiral superfields is a left-chiral superfield itself. Therefore, if the quantity $\mathcal{W}\left(\Phi_{i}\right)$ is a holomorphic function of left-chiral superfields $\Phi_{i}$, then it is a left-chiral superfield itself and it produces supersymmetry-invariant terms as

$$
\left.\mathcal{W}\left(\Phi_{1}, \Phi_{2}, \ldots\right)\right|_{F}
$$

which, in terms of its component fields, reads

$$
\left.\mathcal{W}\left(\Phi_{i}\right)\right|_{F}=\frac{\partial \mathcal{W}\left(\phi_{i}\right)}{\partial \phi_{j}} F_{j}-\frac{1}{2} \frac{\partial^{2} \mathcal{W}\left(\phi_{i}\right)}{\partial \phi_{j} \partial \phi_{k}} \chi_{i} \cdot \chi_{j}+\text { h.c. }
$$

and it is what we call a superpotential. It turns out that the interaction terms of the minimal supersymmetric (Wess-Zumino) model can be obtained from the superpotential terms

$$
\mathcal{L}_{i n t}^{W Z}=\left.\left(\frac{1}{2} m_{i j} \Phi_{i} \Phi_{j}+\frac{1}{3 !} y_{i j k} \Phi_{i} \Phi_{j} \Phi_{k}\right)\right|_{F}+\text { h.c. }
$$

The kinetic terms, on the other hand, are obtained as the coefficients of the term with $\theta \cdot \theta \bar{\theta} \cdot \bar{\theta}$ from

$$
\mathcal{L}_{k i n}^{W Z}=\int \mathrm{d}^{4} \theta \Phi^{\dagger} \Phi=\left.\Phi^{\dagger} \Phi\right|_{D}
$$

which is a supersymmetry-invariant quantity that we call a $D$-term, a name that will acquire its meaning in the next subsection, where we introduce the vector superfield.

\section{A.2 Supersymmetric gauge theory}

Introducing a $\mathrm{U}(1)$ phase on the chiral superfields as

$$
\Phi \longrightarrow e^{2 i q \Lambda} \Phi
$$

where $q$ is the charge, we understand that $\Lambda(x)$ must be a chiral superfield as well, since a phase cannot change the chirality of a superfield (otherwise it is not a phase). As far as 
the kinetic term 1.15 is concerned, it acquires a phase $\Phi^{\dagger} \Phi \longrightarrow e^{2 i q\left(\Lambda-\Lambda^{\dagger}\right)} \Phi^{\dagger} \Phi$ which is compensated by the introduction of a superfield $\mathcal{V}$ as $\Phi^{\dagger} e^{2 q \mathcal{V}} \Phi$, which transforms under a gauge transformation as

$$
\mathcal{V} \longrightarrow \mathcal{V}-i\left(\Lambda-\Lambda^{\dagger}\right)
$$

Observing in the above expression that $\mathcal{V}$ transforms into real gauges, we can choose it to be a real superfield, i.e. $\mathcal{V}=\mathcal{V}^{\dagger}$. Given that, we can restraint $\mathcal{V}$ to the Wess-Zumino (WZ) gauge as

$$
\mathcal{V}=-\theta \sigma^{\mu} \bar{\theta} A_{\mu}+\theta \cdot \theta \bar{\theta} \cdot \bar{\zeta}-i \bar{\theta} \cdot \bar{\theta} \theta \cdot \zeta+\frac{1}{2} \theta \cdot \theta \bar{\theta} \cdot \bar{\theta} D
$$

which is what we call the vector (gauge) superfield. In WZ gauge, there is still one redundant gauge freedom left, $\Lambda(x)=a(x)$, which is the ordinary freedom of the spacetime gauge field $A_{\mu}(x) \longrightarrow A_{\mu}(x)+\partial_{\mu} a(x)$. Since we are dealing with a $\mathrm{U}(1)$ theory and we have already introduced a gauge field through the coupling to the vector superfield, the action is gauge invariant as it is (the covariant derivative $\mathcal{D}_{\mu}$ naturally forms for the $\phi$ and $\chi$ inside $\Phi$, on the expanded kinetic action) while supersymmetry is not affected by the gauge freedom.

Note that last argument: for Abelian gauge transformations, supersymmetry does not conflict with gauge freedom. We can see that on the level of the supersymmetric variations, which include the field strength $F_{\mu \nu}$ that is gauge invariant under $\mathrm{U}(1)$. This also means that, while $\Phi$ (if present) is charged under $\mathrm{U}(1)$, the spinor $\lambda$ inside the vector superfield $(1.18)$ is not $\mathrm{U}(1)$-charged. In the non-Abelian case things must be modified, since $F_{\mu \nu}$ is not gauge invariant anymore and thus the spinor and the auxiliary field must also transform under the gauge transformations in order to compensate and leave the supersymmetric variations gauge invariant. This is achieved by just advancing all the (super) derivatives into (super) covariant derivatives $\mathcal{D}_{\alpha}, \overline{\mathcal{D}}_{\dot{\alpha}}$ and $\mathcal{D}_{\mu}$.

Except the new kinetic term for the left-chiral superfields,

$$
\left.\Phi_{i}^{\dagger} e^{2 q_{i} \mathcal{V}} \Phi_{i}\right|_{D}
$$

gauge invariance of the action must also hold for their superpotential, 1.13). Therefore, since the superpotential is a holomorphic function of the chiral superfields, then it can only be gauge-invariant if

$$
\sum_{i} q_{i}=0
$$


The only thing left is to construct the field strength of the Abelian vector superfield, that gives the kinetic terms its component fields. The strength reads

$$
\mathcal{F}_{\alpha} \equiv \bar{D} \cdot \bar{D} D_{\alpha} \mathcal{V}
$$

which, observing that $\bar{D}_{\dot{\alpha}} \mathcal{F}_{\beta}=0$, is a spinor (anticommuting) left-chiral superfield. Being a left-chiral superfield, it naturally builds the supersymmetry-invariant kinetic $F$-term

$$
\left.\frac{1}{4} \mathcal{F} \cdot \mathcal{F}\right|_{F}
$$

which expands in the usual kinetic terms for component fields of the gauge multiplet 1.18 .

\section{B $\mathcal{N}=(0,2)$ superfields in two dimensions}

Superspace in $d=1+1$ dimensions is parametrized by the spacetime coordinates $x^{ \pm}=$ $x^{0} \pm x^{1}$ and the complex (one-component ${ }^{1}$ ) Grassmann coordinates $\theta^{ \pm}, \bar{\theta}^{ \pm}$. The latter can be realized as the reduction of the anticommuting coordinates in four dimensions, which are two-component Weyl spinors, as

$$
\theta_{\alpha} \equiv\left(\begin{array}{c}
\theta^{1} \\
\theta^{2}
\end{array}\right) \stackrel{K K}{\longrightarrow}\left\{\begin{array}{l}
\theta^{+} \\
\theta^{-}
\end{array}\right.
$$

and their complex conjugates. While the four-dimensional $\theta_{\alpha}$ coordinate is Weyl, i.e. a chiral spinor, its reduced two-dimensional children $\theta^{ \pm}$have opposite chiralities. This antithesis is insightful in the context of the dimensionally reduced R-symmetry, which we analyze in the last section.

$\mathcal{N}=(0,2)$ superspace is the restriction of life on the single right-moving complex coordinate $\theta^{+}$and its conjugate $\bar{\theta}^{+}, 154-156$. The supercharges are always chosen to be minimal spinors, i.e. one-component real Majorana-Weyl spinors in two spacetime dimensions, which means that the amount of supersymmetry $\mathcal{N}$ also represents the number of the supercharges. Thus, in this case we have the real chiral supercharges

$$
Q_{+}=\frac{\partial}{\partial \theta^{+}}+i \bar{\theta}^{+}\left(\partial_{0}+\partial_{1}\right), \quad \bar{Q}_{+}=-\frac{\partial}{\partial \bar{\theta}^{+}}-i \theta^{+}\left(\partial_{0}+\partial_{1}\right)
$$

These commute with

\footnotetext{
${ }^{1}$ In $d$ spacetime dimensions, a Dirac spinor has $2^{\left[\frac{d}{2}\right]}$ complex components.
} 


$$
\mathcal{D}_{+}=\frac{\partial}{\partial \theta^{+}}-i \bar{\theta}^{+}\left(\mathcal{D}_{0}+\mathcal{D}_{1}\right), \quad \overline{\mathcal{D}}_{+}=-\frac{\partial}{\partial \bar{\theta}^{+}}+i \theta^{+}\left(\mathcal{D}_{0}+\mathcal{D}_{1}\right)
$$

where $\mathcal{D}_{0}+\mathcal{D}_{1}=\partial_{0}+\partial_{1}+i A_{0}+i A_{1}$, that is we introduced (super) covariant derivatives to handle with a non Abelian theory from now on. These derivatives obey

$$
\overline{\mathcal{D}}_{+}^{2}=\mathcal{D}_{+}^{2}=0, \quad\left\{\mathcal{D}_{+}, \overline{\mathcal{D}}_{+}\right\}=2 i\left(\mathcal{D}_{0}+\mathcal{D}_{1}\right)
$$

Both $\mathcal{N}=(0,2)$ and $\mathcal{N}=(0,4)$ supersymmetry algebras have the feature of containing only right-moving supercharges. This means that right-handed spinors are paired with bosonic fields in the familiar supersymmetric manner. In contrast, left-moving spinors can be loners; they need not have bosonic companions, in which case supersymmetry acts only to restrict their interactions with the right-handed spinors.

Therefore, two-dimensional theories with $\mathcal{N}=(m, n)$ supersymmetry exhibit an $\mathrm{SO}(m)_{R} \times \mathrm{SO}(n)_{R}$ $\mathrm{R}$-symmetry, which rotates left and right-moving supercharges respectively. Thus a $\mathcal{N}=$ $(0,2)$ theory has a $\mathrm{U}(1)_{R}$ R-symmetry, under which $\theta^{+}$has charge +1 and $\bar{\theta}^{+}$has -1 . Rsymmetry is important since its R-current anomaly is directly associated with the central charge of the theory. The different R-symmetry realizations are discussed in Section $\mathrm{D}$.

\section{B.1 Gauge multiplet}

The gauge multiplet is a real superfield, $\mathcal{V}$. It comprises of a gauge field $A$, an adjoint-valued complex left-handed fermion $\zeta_{-}$and a real auxiliary field $D$. The component expansion is

$$
\mathcal{V}=\left(A_{0}-A_{1}\right)-i \theta^{+} \bar{\zeta}_{-}-i \bar{\theta}^{+} \zeta_{-}+\theta^{+} \bar{\theta}^{+} D
$$

where the bosonic and fermionic degrees of freedom (dof) do not have to match (and they don't), since the right-moving supercharges of $\mathcal{N}=(0,2)$ supersymmetry does not act on left-handed spinors.

Having introduced the two-dimensional vector superfield, we can also define the lefthanded super covariant derivative

$$
\mathcal{D}_{-}=\mathcal{D}_{0}-\mathcal{D}_{1}=\partial_{0}-\partial_{1}-i \mathcal{V},
$$

which may be used to write down the kinetic term for a chiral superfield and the superfield 
strength

$$
\Upsilon=\left[\overline{\mathcal{D}}_{+}, \mathcal{D}_{-}\right]=-\zeta_{-}-i \theta^{+}\left(D-i F_{01}\right)-i \theta^{+} \bar{\theta}^{+}\left(\mathcal{D}_{0}+\mathcal{D}_{1}\right) \zeta_{-}
$$

This satisfies $\overline{\mathcal{D}}_{+} \Upsilon=0$, which means that $\Upsilon$ is a spinor left-chiral superfield. Equivalently, it is a special case of a Fermi multiplet, which will be introduced below.

The standard kinetic action for the gauge multiplet reads

$$
\begin{aligned}
S_{\text {gauge }} & =\frac{1}{8 g^{2}} \operatorname{Tr} \int \mathrm{d}^{2} x \mathrm{~d}^{2} \theta \bar{\Upsilon} \Upsilon \\
& =\frac{1}{g^{2}} \operatorname{Tr} \int \mathrm{d}^{2} x\left(\frac{1}{2} F_{01}^{2}+i \bar{\zeta}_{-}\left(\mathcal{D}_{0}+\mathcal{D}_{1}\right) \zeta_{-}+D^{2}\right)
\end{aligned}
$$

\section{B.2 Chiral multiplet}

$\mathcal{N}=(0,2)$ chiral multiplets contain a right-moving fermion $\psi_{+}$and a single complex scalar $\phi$, each transforming in the same representation of the gauge group. These synthesize a complex-valued bosonic chiral superfield $\Phi$ obeying

$$
\overline{\mathcal{D}}_{+} \Phi=0
$$

with component expansion

$$
\Phi=\phi+\theta^{+} \psi_{+}-i \theta^{+} \bar{\theta}^{+}\left(\mathcal{D}_{0}+\mathcal{D}_{1}\right) \phi
$$

Note, here, the matching between the bosonic and fermionic dof. Since $\psi_{+}$is one-component, the Dirac equation may still be a matrix equation but there is no mixing between spinor components.

The kinetic terms for the gauged chiral multiplet are now given by

$$
\begin{aligned}
S_{\text {chiral }} & =\int \mathrm{d}^{2} x \mathrm{~d}^{2} \theta \bar{\Phi}\left(\mathcal{D}_{0}-\mathcal{D}_{1}\right) \Phi \\
& =\int \mathrm{d}^{2} x\left(-\left|D_{\mu} \phi\right|^{2}+i \bar{\psi}_{+}\left(D_{0}-D_{1}\right) \psi_{+}-i \bar{\phi} \zeta_{-} \psi_{+}+i \bar{\psi}_{+} \bar{\zeta}_{-} \phi+\bar{\phi} D \phi\right) .
\end{aligned}
$$




\section{B.3 Fermi multiplet}

$\mathcal{N}=(0,2)$ theories have the property that left-moving spinors are not necessarily accompanied by propagating, bosonic superpartners. A spinor of this kind, $\psi_{-}$, sits in an anticommuting superfield $\Psi$, obeying the condition

$$
\overline{\mathcal{D}}_{+} \Psi=E,
$$

where, considering 1.26 and $\overline{\mathcal{D}}_{+}^{2}=0, E$ is some chiral superfield

$$
\overline{\mathcal{D}}_{+} E=0 \text {. }
$$

We call $\Psi$ a Fermi multiplet. In our context, we always take $E=E\left(\Phi_{i}\right)$ to be a holomorphic function of the chiral superfields $\Phi_{i}$. This function must be chosen so that $E\left(\Phi_{i}\right)$ transforms in the same manner as $\Psi$ under any symmetries; we will realize this in the context of Rsymmetry, below. Being a chiral superfield, $E$ expands as

$$
E\left(\Phi_{i}\right)=E\left(\phi_{i}\right)+\theta^{+} \frac{\partial E}{\partial \phi_{i}} \psi_{+i}-i \theta^{+} \bar{\theta}^{+}\left(D_{0}+D_{1}\right) E\left(\phi_{i}\right)
$$

The choice of $E$ determines the interaction of the theory and also appears in the component expansion of the superfield,

$$
\Psi=\psi_{-}-\theta^{+} G-i \theta^{+} \bar{\theta}^{+}\left(D_{0}+D_{1}\right) \psi_{-}-\bar{\theta}^{+} E\left(\phi_{i}\right)+\theta^{+} \bar{\theta}^{+} \frac{\partial E}{\partial \phi_{i}} \psi_{+i}
$$

Here $G$ is a complex auxiliary field. As mentioned before, the superfield strength $\Upsilon$ is of this type, with $\overline{\mathcal{D}}_{+} \Upsilon=(E=) 0$.

The kinetic terms for the Fermi multiplet are given by

$$
\begin{aligned}
S_{\mathrm{Fermi}} & =\int \mathrm{d}^{2} x \mathrm{~d}^{2} \theta \bar{\Psi} \Psi \\
& =\int \mathrm{d}^{2} x\left(i \bar{\psi}_{-}\left(D_{0}+D_{1}\right) \psi_{-}+|G|^{2}-\left|E\left(\phi_{i}\right)\right|^{2}-\bar{\psi}_{-} \frac{\partial E}{\partial \phi_{i}} \psi_{+i}+\bar{\psi}_{+i} \frac{\partial \bar{E}}{\partial \bar{\phi}_{i}} \psi_{-}\right) .
\end{aligned}
$$


Note that the holomorphic function $E\left(\phi_{i}\right)$ appears as potential terms in the Lagrangian. Thus, just by constraining the Fermi superfield $\Psi$ on the chiral superfields $\Phi_{i}$, as in (1.34), we acquire interactions. However, as we now explain, this is not the only way to introduce potential terms though chiral multiplets.

\section{B.4 Superpotentials}

Each Fermi multiplet contains an auxiliary complex scalar $G$, which transforms as a total derivative. Thus, we may consider the supersymmetric $G$-terms

$$
S \sim \int \mathrm{d} \theta^{+} \Psi
$$

which however is uninteresting. To spice things up, we notice that, like the $F$-terms of the scalar chiral superfields are supersymmetry-invariant, so are the $G$-terms of anticommuting chiral superfields $2^{2}$,

$$
\left.\int \mathrm{d}^{2} x \mathrm{~d} \theta^{+}(\ldots)\right|_{\bar{\theta}^{+}=0}+\text { h.c. }
$$

where $(\ldots)$ is an anticommuting superfield, annihilated by $\overline{\mathcal{D}}_{+}$. Given that a product of chiral superfields is itself a chiral superfield and, also, that a product of an anticommuting and a commuting (scalar) superfield is itself an anticommuting superfield ${ }^{3}$, an obvious candidate for $(\ldots)$ is

$$
\left.\int \mathrm{d}^{2} x \mathrm{~d} \theta^{+}(\Psi J)\right|_{\bar{\theta}^{+}=0}+\text { h.c. }
$$

where $J=J\left(\Phi_{i}\right)$ is a holomorphic function of the $\Phi_{i}$ 's, while $\overline{\mathcal{D}}_{+} \Psi=\overline{\mathcal{D}}_{+} J=0$. But this only works for the special case of a chiral Fermi superfield, i.e. with $E=0$. Therefore, we consider multiple Fermi superfields $\Psi_{a}$ corresponding to multiple scalar chiral superfields $J^{a}$, together building the sum $\sum_{a} \Psi_{a} J^{a}$ that has a supersymmetric $G$-term only when it is a chiral superfield, that is iff

$$
\overline{\mathcal{D}}_{+}\left(\Psi_{a} J^{a}\right)=0
$$

\footnotetext{
${ }^{2}$ In the special case of $11.39, \Psi$ does not have to be chiral, i.e. $\overline{\mathcal{D}}_{+} \Psi=E=0$, for this $G$-term to be supersymmetric. That is, we only extract a single auxiliary field $G$ which transforms as a total derivative on its own right.

${ }^{3}$ Following the obvious rule: odd $\cdot$ even $=$ odd.
} 
This, in turn, is satisfied if

$$
E \cdot J \equiv \sum_{a} E_{a} J^{a}=0
$$

which constitutes a highly strong constraint for a supersymmetric theory. This shows that there is some tension when introducing both $E$-type potentials and $J$-type potentials associated to the same Fermi multiplet. If we only have one Fermi multiplet, then this necessarily means that $(J \neq 0$ and) $E=0$, as we argued for $(1.41)$. In general, however, $E \neq 0$ if we can arrange some cancellation between other Fermi multiplets. This condition will prove crucial in our construction of $\mathcal{N}=(0,4)$ theories below.

Overall, we obtain the supersymmetric term

$$
S_{J}=\left.\int \mathrm{d}^{2} x \mathrm{~d} \theta^{+} \sum_{a} \Psi_{a} J^{a}\left(\Phi_{i}\right)\right|_{\bar{\theta}^{+}}+\text {h.c. }
$$

which expands in

$$
S_{J}=\sum_{a} \int \mathrm{d}^{2} x\left(G_{a} J^{a}\left(\phi_{i}\right)+\sum_{i} \psi_{-a} \frac{\partial J^{a}}{\partial \phi_{i}} \psi_{+i}\right)+\text { h.c. . }
$$

After integrating out the auxiliary fields $G_{a}$, this results in a potential term $\sim\left|J^{a}\left(\phi_{i}\right)\right|^{2}$. Notice that the fields indexed by $i$ all come from the function $J^{a}\left(\Phi_{i}\right)$. 11.45) is usually referred to as the superpotential in $\mathcal{N}=(0,2)$ theories. In what follows, we will also use the notation

$$
\mathcal{W}=\Psi_{a} J^{a}(\Phi)
$$

Hence, we see that there are two ways to construct potential terms in theories with $\mathcal{N}=(0,2)$ supersymmety. Both are associated to Fermi multiplets and both involve holomorphic functions, $E\left(\phi_{i}\right)$ and $J\left(\phi_{i}\right)$. The difference between them is not visible in the bosonic Lagrangian alone; it only shows through the subtle difference in the Yukawa terms in $(1.38)$ and $(1.45)$. This difference will be important in the context of $\mathcal{N}=(0,4)$ theories. 


\section{B.5 $\mathcal{N}=(2,2)$ decomposition}

The $\mathcal{N}=(0,2)$ vector superfield may also be realized as the dimensional reduction of the $\mathcal{N}=$ 1 four-dimensional vector superfield (1.18). The latter reduces into a two-dimensional $\mathcal{N}=$ $(2,2)$ vector superfield $]^{4}$ (a $\mathcal{N}=4$ multiplet in two dimensions), which in turn breaks under $\mathcal{N}=(0,2)$ supersymmetry into the vector multiplet 1.27$)$ with the non supersymmetric left-moving spinor $\zeta_{-}$and the $\mathcal{N}=(0,2)$ chiral multiplet 1.32 with the supersymmetric right-moving $\zeta_{+}$.

The $\mathcal{N}=1$ four-dimensional chiral superfield 1.10 reduces into a two-dimensional $\mathcal{N}=(2,2)$ chiral superfield 5 which in turn breaks under $\mathcal{N}=(0,2)$ supersymmetry into the chiral multiplet 1.32 with the supersymmetric right-moving spinor $\psi_{+}$and a $\mathcal{N}=(0,2)$ Fermi multiplet (1.37) with the non supersymmetric left-moving $\psi_{-}$.

\section{C $\mathcal{N}=(0,4)$ superfields in two dimensions}

Theories in two dimensions with $\mathcal{N}=(0,4)$ supersymmetry have four, real, right-moving supercharges. Correspondingly, they have an R-symmetry

$$
\mathrm{SO}(4)_{R} \cong \mathrm{SU}(2)_{R}^{-} \times \mathrm{SU}(2)_{R}^{+}
$$

The supercharges transform in the $(\mathbf{2}, \mathbf{2})+$ representation, where the subscript denotes their chirality under the $\mathrm{SO}(1,1)$ Lorentz group.

We now move one to build $\mathcal{N}=(0,4)$ theories using $\mathcal{N}=(0,2)$ superfields. These theories are composed so that they enjoy an enhanced $\mathrm{SO}(4)_{R}$ R-symmetry, which ensures that there is an extended supersymmetry.

\section{C.1 Vector multiplets}

Since in two dimensions the gauge field is not propagating it is natural that two-dimensional $\mathcal{N}=(0,4)$ vector multiplets are composed of left-handed spinors, which don't transform under supersymmetry ${ }^{6}$. We first indicated this for the $\mathcal{N}=(0,2)$ vector superfields 1.27). Thus, a $\mathcal{N}=(0,4)$ vector superfield consists of a $\mathcal{N}=(0,2)$ vector and an adjoint-valued

\footnotetext{
${ }^{4}$ This comprises of a real auxiliary field $D$, a complex scalar $\phi$ and two complex fermions $\zeta_{ \pm}$that lose half of their dof under their common Dirac equation.

${ }^{5}$ This comprises of a complex auxiliary field $F$, a complex scalar $\phi$ and two complex fermions $\psi_{ \pm}$that lose half of their dof under their common Dirac equation.

${ }^{6}$ A two-dimensional $\mathcal{N}=(0,4)$ vector superfield has no right-handed spinors, since these would transform under supersymmetry while their multiplet has no on-shell bosonic dof.
} 
$\mathcal{N}=(0,2)$ Fermi multiplet $\Theta$. Besides the gauge field, there are a pair of left moving complex fermions, $\zeta_{-}^{a}, a=1,2$, transforming as $(\mathbf{2}, \mathbf{2})-7$ under the R-symmetry and a triplet of auxiliary fields transforming as $(\mathbf{3}, \mathbf{1})^{8}$

The Fermi superfield obeys

$$
\overline{\mathcal{D}}_{+} \Theta=E_{\Theta},
$$

where $E_{\Theta}$ depends on the matter content, i.e. the chiral superfields present in the theory. The $\mathcal{N}=(0,4)$ action is obviously given by the sum of 1.30 and 1.38 .

\section{C.2 Hypermultiplets and twisted hypermultiplets}

There are two distinct ways to couple matter fields to a $\mathcal{N}=(0,4)$ vector multiplet (essentially to its constituent $\mathcal{N}=(0,2)$ Fermi multiplet). For this reason, we distinguish between hypermultiplets and twisted hypermultiplets.

An $\mathcal{N}=(0,4)$ hypermultiplet consists of a pair of $\mathcal{N}=(0,2)$ chiral multiplets, $\Phi$ and $\tilde{\Phi}$ , transforming in conjugate representations of the gauge group. The pair of complex scalars transforms as $(\mathbf{2}, \mathbf{1})$ under the R-symmetry, while the pair of right-handed spinors transforms as $(\mathbf{1}, \mathbf{2})_{+}$.

The kinetic terms for both chiral multiplets are given by (1.33). In addition, there is a coupling to Fermi superfield in the $\mathcal{N}=(0,4)$ vector multiplet. This takes the form of a superpotential (1.46) with

$$
J_{\Theta}=\Phi \tilde{\Phi} \quad \Rightarrow \quad \mathcal{W}_{\Theta}=\Phi \Theta \tilde{\Phi}
$$

The $\mathcal{N}=(0,4)$ twisted hypermultiplet also consists of a pair of $\mathcal{N}=(0,2)$ chiral multiplets, $\Phi^{\prime}$ and $\tilde{\Phi}^{\prime}$, transforming in conjugate representations of the gauge group. The differ-

\footnotetext{
${ }^{7}$ Naively, the left-handed spinors which are singlets under the right-moving supersymmetry would also be singlets under the R-symmetry. However, since both the $\mathcal{N}=(0,2)$ vector and the Fermi multiplets couple to chiral superfields, as in 1.33 and 1.38 , that do transform under R-symmetry, the former must transform as well for an R-symmetric theory. As we will see below, the reason that these left-handed spinors transform under both $\mathrm{SU}(2)$ 's is that the various chiral multiplets (to which they couple) transform under both groups. Another starting point could be the supersymmetric variations. There, by requiring that the supersymmetry-variation parameters $\epsilon^{ \pm}$are charged appropriately, we find the R-charges of the component fields. This procedure however may be bypassed, since we know that the gauge field - and thus the vector superfield - are not charged under R-symmetry and so we can require $R[\Upsilon]=+1$.

${ }^{8}$ The $\mathcal{N}=(0,2)$ vector and Fermi multiplets together have 3 real auxiliary dof, which transform under one of the $\mathrm{SO}(3)_{R} \cong \mathrm{SU}(2)_{R}$.
} 
ence from the hypermultiplet lies in the R-symmetry transformation of the fields. The pair of scalars transform as $(\mathbf{1}, \mathbf{2})$ while the pair of right-moving fermions transforms as $(\mathbf{2}, \mathbf{1})_{+}$.

The kinetic terms for the two chiral multiplets are again given by (1.33). The different R-symmetry transformations are enforced by the coupling to the Fermi field $\Theta$. In contrast to the hypermultiplet, the coupling is no longer through the superpotential but instead via the relation 1.48 , with

$$
E_{\Theta}=\Phi^{\prime} \tilde{\Phi}^{\prime}
$$

with the combination $\Phi^{\prime} \tilde{\Phi}^{\prime}$ arranged so that it transforms in the adjoint of the gauge group.

To realize why the hypermultiplet and the twisted hypermultiplet transform differently, we just observe their coupling on the level of the action in 1.45). There, the Fermi multiplet (which couples to the $E$ function) couples to the $J$ function. Requiring the action to be R-symmetric under an operator $R$, we find that $R[\Phi]=1-R\left[\Phi^{\prime}\right]$, where $\Phi, \Phi^{\prime}$ represent chiral superfields coming from hypermultiplets and twisted hypermultiplets, respectively. This means that whenever a hypermultiplet is charged as +1 the other is a singlet and vice versa.

To preserve $\mathcal{N}=(0,2)$ supersymmetry, we must have $E \cdot J=0$. If we have only a single vector multiplet, this condition reads

$$
E_{\Theta} J^{\Theta}=0
$$

Obviously, as we commented when we introduced the superpotential, this is not satisfied if we naively try to couple both hypermultiplets and twisted hypermultiplets to the same gauge group (except if the Fermi multiplet is a chiral superfield, i.e. $E_{\Theta}=0$, which is not the case here). To do this in a manner consistent with supersymmetry, we need to introduce further multiplets.

\section{C.3 Fermi Multiplets}

We define the $\mathcal{N}=(0,4)$ Fermi multiplets to consist of a pair of $\mathcal{N}=(0,2)$ Fermi multiplets, $\Gamma$ and $\tilde{\Gamma}$, transforming in conjugate representations of the gauge group. The left-handed spinors transform as $(\mathbf{1}, \mathbf{1})_{\text {- }}$ under the R-symmetry?

\footnotetext{
${ }^{9}$ In contrast with the Fermi multiplet $\Theta$ inside the $\mathcal{N}=(0,4)$ vector superfield, the Fermi multiplets $\Gamma, \tilde{\Gamma}$ couple, as we see below, in a mixed manner with the hyper and twisted hypermultiplets. This mixing cancels the R-charges between them, in the level of the action, applying no pressure anymore to the Fermi multiplets to be R-charged (in order for the action to be R-symmetric). Thus, these Fermi's are singlets under R-symmetry.
} 
The kinetic terms for each fermion are given by 1.38 ; no further coupling between $\Gamma, \tilde{\Gamma}$ and $\Theta$ is needed (nor, indeed, possible, since both contain left-moving fermions only). It is, however, possible to introduce other couplings for the Fermi multiplet through the potentials $E$ and $J$ in a manner that preserves $\mathrm{SO}(4)_{R}$ R-symmetry. While we have not determined the most general such interaction, the one that will be relevant for our purposes couples a Fermi multiplet to a hypermultiplet and a twisted hypermultiplet. It arises, as we explain shortly, when we look at the better studied $\mathcal{N}=(4,4)$ gauge theories through $\mathcal{N}=(0,4)$ eyes.

Finally, we note that it is possible to have a single $\mathcal{N}=(0,2)$ Fermi multiplet which is consistent with $\mathcal{N}=(0,4)$ supersymmetry. For this to happen, the chiral fermion should be a singlet under the $\mathrm{SO}(4)_{R}$ R-symmetry. Of course, the coupling to other matter multiplets must also respect this.

\section{C.4 $\mathcal{N}=(4,4)$ Decomposition}

To understand $\mathcal{N}=(0,4)$ theories better, it is useful to see how $\mathcal{N}=(4,4)$ multiplets decompose into their $\mathcal{N}=(0,4)$ counterparts.

The $\mathcal{N}=(4,4)$ vector multiplet splits into an $\mathcal{N}=(0,4)$ vector multiplet and an adjointvalued $\mathcal{N}=(0,4)$ twisted hypermultiplet. We will denote the $\mathcal{N}=(0,2)$ chiral multiplets in this twisted hypermultiplet as $\Sigma$ and $\tilde{\Sigma}$. They couple to the Fermi mutliplet $\Theta$ (inside the $\mathcal{N}=(0,4)$ vector superfield) through,

$$
E_{\Theta}=[\Sigma, \tilde{\Sigma}]
$$

An $\mathcal{N}=(4,4)$ hypermultiplet decomposes into an $\mathcal{N}=(0,4)$ hypermultiplet, $\Phi$ and $\tilde{\Phi}$, and an $\mathcal{N}=(0,4)$ Fermi multiplet, $\Gamma$ and $\tilde{\Gamma}$. As described above, there is a superpotential term

$$
\mathcal{W}_{\Theta}=\tilde{\Phi} \Theta \Phi
$$

The remaining couplings are associated to the $\mathcal{N}=(0,4)$ Fermi multiplet and provide the example of an interaction between a Fermi multiplet $\Gamma, \tilde{\Gamma}$, a hypermultiplet $\Phi, \tilde{\Phi}$ and a twisted hypermultiplet $\Sigma, \tilde{\Sigma}$ that we promised above. The interaction makes use of both superpotentials and $E$ terms. The former are given by

$$
\mathcal{W}_{\tilde{\Gamma}}+\mathcal{W}_{\Gamma}=\tilde{\Gamma} \tilde{\Sigma} \Phi+\tilde{\Phi} \tilde{\Sigma} \Gamma
$$


These are combined with the $E$-term couplings

$$
E_{\Gamma}=\Sigma \Phi \quad E_{\tilde{\Gamma}}=-\tilde{\Phi} \Sigma .
$$

Note that the constraint $(1.43)$ is satisfied, as required by supersymmetry, since

$$
E \cdot J=(\tilde{\Phi}[\Sigma, \tilde{\Sigma}] \Phi+\tilde{\Phi} \tilde{\Sigma} \Sigma \Phi-\tilde{\Phi} \Sigma \tilde{\Sigma} \Phi)=0
$$

\section{R-symmetry}

R-symmetry is the internal symmetry of a superalgebra, under which the supercharges rotate into each other. In the level of the action, where the superalgebra is realized through fields, R-symmetry manifests as a global symmetry on those fields. Therefore, in general, a supersymmetric theory with $\mathcal{N}$ supersymmetries exhibits at most a $\mathrm{U}(\mathcal{N})$ R-symmetry 10,160$]$. If the supercharges are real (Majorana or Majorana-Weyl), then obviously R-symmetry is realized as $\mathrm{SO}(\mathcal{N})$.

The most familiar example of $\mathrm{R}$-symmetry is for a $\mathcal{N}=1$ supersymmetric four-dimensional theory, where it rotates the supercharges by a $\mathrm{U}(1)$ factor as

$$
[Q, R]=Q, \quad[\bar{Q}, R]=-\bar{Q},
$$

keeping $\{Q, \bar{Q}\}=2 i \sigma^{\mu} P_{\mu}$ invariant. Thus, the standard $\mathrm{U}(1) \mathrm{R}$-symmetry is chiral. Since, in superspace, the supercharges generate a supersymmetric translation of the form

$$
\begin{aligned}
x^{\prime \mu} & =x^{\mu}+a^{\mu}+i \epsilon \sigma^{\mu} \bar{\theta}-i \theta \sigma^{\mu} \bar{\epsilon}, \\
\theta^{\prime} & =\theta+\epsilon, \\
\bar{\theta}^{\prime} & =\bar{\theta}+\bar{\epsilon},
\end{aligned}
$$

then $\mathrm{U}(1)$-charged supercharges imply charged supersymmetric parameters $\epsilon, \bar{\epsilon}$ and grassmann coordinates $\theta, \bar{\theta}$ as well. Therefore, if $Q$ and $\bar{Q}$ are charged with +1 and -1 , then it follows that $\epsilon, \theta$ and $\bar{\epsilon}, \bar{\theta}$ also have charges +1 and -1 , respectively. Assigning these charges

\footnotetext{
${ }^{10}$ For the special case of $\mathcal{N}=4$ in four dimensions, the $\mathrm{U}(1)$ subalgebra commutes with the supercharges and thus R-symmetry reduces to $\mathrm{SU}(\mathcal{N})$.
} 
on the supersymmetric variations of the fields of the theory, we may find the R-charges of these fields.

Nonetheless, R-symmetry also manifests through the dimensional reduction of a supersymmetric theory. This is because the reduced spatial dimensions are realized as fields in the reduced theory, while the previously rotational-symmetry group now becomes a global symmetry rotating those fields. A standard example is the ten-dimensional $\mathcal{N}=1 \mathrm{SYM}$ which reduces to the four-dimensional $\mathcal{N}=4 \mathrm{SYM}$. There, the six reduced spatial dimensions become six scalar fields and the original rotational $\mathrm{SO}(6)$ symmetry becomes a global symmetry rotating the reduced scalar fields. This is another way to realize the $\mathrm{SU}(4) \cong$ $\mathrm{SO}(6)$ R-symmetry of the four-dimensional $\mathcal{N}=4 \mathrm{SYM}$.

Hence, if we dimensionally reduce a theory we may consider two different realizations of R-symmetry. First, it is the original R-symmetry of the unreduced theory that is inherited to the lower-dimensional one. Secondly, it is the R-symmetry that manifests as a realization of the previously rotational group of the reduced spatial dimensions. These are two different realizations of R-symmetry and their distinction manifests in the way they act on the Grassmann dimensions $\theta, \bar{\theta}$ (and on $\epsilon, \bar{\epsilon}$ ).

Let us ground all this on the example of our two-dimensional $\mathcal{N}=(0,4)$ theory. This theory (actually, its $\mathcal{N}=(2,2)$ sibling) is obtained from dimensional reduction on fourdimensional $\mathcal{N}=1$ gauge theory. In four dimensions the $\mathrm{U}(1) \mathrm{R}$-symmetry acts on $\theta, \bar{\theta}$ with charges $+1,-1$ and thus when the spinors get dimensionally reduced as

$$
\theta_{\alpha} \equiv\left(\begin{array}{c}
\theta^{1} \\
\theta^{2}
\end{array}\right) \stackrel{K K}{\longrightarrow}\left\{\begin{array}{l}
\theta^{+} \\
\theta^{-}
\end{array}\right.
$$

then obviously $\theta^{ \pm}, \bar{\theta}^{ \pm}$are also charged with +1 and -1 , respectively.

The second kind of R-symmetry is identified with the $\mathrm{SO}(2)$ rotational group of the two reduced spatial dimensions. In the reduced theory it interchanges the scalars that popped up from dimensionally reducing the gauge field. But how does this R-symmetry acts on spinors (i.e. on supercharges and $\theta, \bar{\theta}$ ) of our reduced theory? The answer lies, of course, on the way $\mathrm{SO}(2)$ acts - as a group of spatial rotations - on the spinors of the unreduced theory.

The situation would be simpler if we were dealing with the reduction of a six-dimensional theory to a two-dimensional one, since then we would have a $\mathrm{SO}(4) \cong \mathrm{SU}(2) \times \mathrm{SU}(2) \mathrm{R}$ symmetry - remnant of the four reduced spatial dimensions - where the left and rightmoving spinors transform independently under the two distinct SU(2)'s.

However, when the 'remnant' R-symmetry is not a decomposable group, like in our $\mathrm{SO}(2)$ case, we have to find how the two spinor chiralities are charged under it. This is simply done by observing the subalgebra (of the unreduced theory) 


$$
\left[Q_{\alpha}, J_{3}\right]=\ldots=+\frac{1}{2} \sigma_{\alpha \beta}^{3} Q_{\beta} \longrightarrow\left\{\begin{array}{l}
{\left[Q_{1}, J_{3}\right]=+\frac{1}{2} Q_{1}} \\
{\left[Q_{2}, J_{3}\right]=-\frac{1}{2} Q_{2}}
\end{array}\right.
$$

where we chose specifically $J_{3}$ which is the helicity operator ${ }^{11}$. This shows that the fourdimensional spinor $\theta_{\alpha}$ has its components $\theta^{1}, \theta^{2}$ charged with +1 and -1 respectively. Equivalently, when we dimensionally reduce as in 1.59 , the reduced Grassmann directions $\theta^{+}, \theta^{-}$ realize opposite R-charges.

Nevertheless, our two-dimensional theory enjoys a $\mathcal{N}=(0,4)$ supersymmetry, which obviously exhibits a $\mathrm{SO}(4)$ right-moving R-symmetry, which in turn rotates the right-moving real supercharges. Focusing on a $\mathrm{U}(1)$ subalgebra of this right-moving R-symmetry, we should be able to obtain it from the previously discussed realizations of R-symmetry. This is achieved by taking a suitable linear combination of those two realizations, in order to construct a right-moving R-symmetry operator, i.e. a $\mathrm{U}(1)$ operator under which $\theta^{+}$is charged with +1 while $\theta^{-}$stays invariant. More details on this can be found in 155 .

\footnotetext{
${ }^{11} J_{3}$ corresponds to $\sigma^{3}$ which is diagonalized in the standard basis and acts on states to reveal their spin. $J_{1}$ and $J_{2}$, on the other hand, mix the spinor components under a rotation.
} 




\section{Bibliography}

[1] K. Filippas, C. Núñez and J. Van Gorsel, JHEP 06 (2019), 069 doi:10.1007/JHEP06(2019)069 [arXiv:1901.08598 [hep-th]].

[2] K. Filippas, JHEP 02 (2020), 027 doi:10.1007/JHEP02(2020)027 [arXiv:1910.12981 [hep-th]].

[3] K. Filippas, Phys. Rev. D 101 (2020) no.4, 046025 doi:10.1103/PhysRevD.101.046025 [arXiv:1912.03791 [hep-th]].

[4] K. Filippas, Phys. Rev. D 103 (2021) no.8, 086003 doi:10.1103/PhysRevD.103.086003 [arXiv:2008.00314 [hep-th]].

[5] S. Weinberg and E. Witten, Phys. Lett. B 96 (1980), 59-62 doi:10.1016/03702693(80)90212-9

[6] M. B. Green, J. H. Schwarz and E. Witten,

[7] M. B. Green, J. H. Schwarz and E. Witten, doi:10.1017/CBO9781139248570

[8] J. Polchinski, Phys. Rev. Lett. 75 (1995), 4724-4727 doi:10.1103/PhysRevLett.75.4724 [arXiv:hep-th/9510017 [hep-th]].

[9] E. Witten, Nucl. Phys. B 443 (1995), 85-126 doi:10.1016/0550-3213(95)00158-O [arXiv:hep-th/9503124 [hep-th]].

[10] A. Sen, Int. J. Mod. Phys. A 9 (1994), 3707-3750 doi:10.1142/S0217751X94001497 [arXiv:hep-th/9402002 [hep-th]].

[11] J. M. Maldacena, Adv. Theor. Math. Phys. 2 (1998), 231-252 doi:10.1023/A:1026654312961 [arXiv:hep-th/9711200 [hep-th]].

[12] S. S. Gubser, I. R. Klebanov and A. M. Polyakov, Phys. Lett. B 428 (1998), 105-114 doi:10.1016/S0370-2693(98)00377-3 [arXiv:hep-th/9802109 [hep-th]]. 
[13] E. Witten, Adv. Theor. Math. Phys. 2 (1998), 253-291 doi:10.4310/ATMP.1998.v2.n2.a2 [arXiv:hep-th/9802150 [hep-th]].

[14] C. B. Thorn, [arXiv:hep-th/9405069 [hep-th]].

[15] C. R. Stephens, G. 't Hooft and B. F. Whiting, Class. Quant. Grav. 11 (1994), 621-648 doi:10.1088/0264-9381/11/3/014 [arXiv:gr-qc/9310006 [gr-qc]].

[16] L. Susskind, J. Math. Phys. 36 (1995), 6377-6396 doi:10.1063/1.531249 [arXiv:hepth/9409089 [hep-th]].

[17] J. D. Bekenstein, Phys. Rev. D 23 (1981), 287 doi:10.1103/PhysRevD.23.287

[18] J. D. Bekenstein, Phys. Rev. D 49 (1994), 1912-1921 doi:10.1103/PhysRevD.49.1912 [arXiv:gr-qc/9307035 [gr-qc]].

[19] A. Strominger and C. Vafa, Phys. Lett. B 379 (1996), 99-104 doi:10.1016/03702693(96)00345-0 [arXiv:hep-th/9601029 [hep-th]].

[20] G. 't Hooft, Nucl. Phys. B 72 (1974), 461 doi:10.1016/0550-3213(74)90154-0

[21] M. Blau, J. M. Figueroa-O'Farrill, C. Hull and G. Papadopoulos, JHEP 01 (2002), 047 doi:10.1088/1126-6708/2002/01/047 [arXiv:hep-th/0110242 [hep-th]].

[22] D. E. Berenstein, J. M. Maldacena and H. S. Nastase, JHEP 04 (2002), 013 doi:10.1088/1126-6708/2002/04/013 [arXiv:hep-th/0202021 [hep-th]].

[23] J. A. Minahan and K. Zarembo, JHEP 03 (2003), 013 doi:10.1088/11266708/2003/03/013 [arXiv:hep-th/0212208 [hep-th]].

[24] N. Beisert, C. Ahn, L. F. Alday, Z. Bajnok, J. M. Drummond, L. Freyhult, N. Gromov, R. A. Janik, V. Kazakov and T. Klose, et al. Lett. Math. Phys. 99 (2012), 3-32 doi:10.1007/s11005-011-0529-2 [arXiv:1012.3982 [hep-th]].

[25] O. Babelon, D. Bernard and M. Talon, "Introduction to classical integrable systems", Cambridge University Press, 2003.

[26] A. Torrielli, J. Phys. A 49 (2016) no.32, 323001 doi:10.1088/1751-8113/49/32/323001 [arXiv:1606.02946 [hep-th]].

[27] O. Lunin and J. M. Maldacena, JHEP 0505, 033 (2005) doi:10.1088/11266708/2005/05/033 [hep-th/0502086].

[28] K. Sfetsos, Nucl. Phys. B 880, 225 (2014) doi:10.1016/j.nuclphysb.2014.01.004 [arXiv:1312.4560 [hep-th]]. 
[29] F. Delduc, M. Magro and B. Vicedo, JHEP 1410, $132 \quad$ (2014) doi:10.1007/JHEP10(2014)132 [arXiv:1406.6286 [hep-th]].

[30] R. Borsato and L. Wulff, Phys. Rev. Lett. 117, no. 25, 251602 (2016) doi:10.1103/PhysRevLett.117.251602 [arXiv:1609.09834 [hep-th]].

[31] Y. Lozano, N. T. Macpherson, C. Nunez and A. Ramirez, JHEP 01 (2020), 129 doi:10.1007/JHEP01(2020)129 [arXiv:1908.09851 [hep-th]].

[32] Y. Lozano, N. T. Macpherson, C. Nunez and A. Ramirez, Phys. Rev. D 101 (2020) no.2, 026014 doi:10.1103/PhysRevD.101.026014 [arXiv:1909.09636 [hep-th]].

[33] Y. Lozano, N. T. Macpherson, C. Nunez and A. Ramirez, JHEP 01 (2020), 140 doi:10.1007/JHEP01(2020)140 [arXiv:1909.10510 [hep-th]].

[34] Y. Lozano, N. T. Macpherson, C. Nunez and A. Ramirez, JHEP 12 (2019), 013 doi:10.1007/JHEP12(2019)013 [arXiv:1909.11669 [hep-th]].

[35] N. Bobev, F. F. Gautason and K. Hristov, Phys. Rev. D 100, no. 2, 021901(R) (2019) doi:10.1103/PhysRevD.100.021901 [arXiv:1903.05095 [hep-th]].

[36] D. Gaiotto and A. Tomasiello, JHEP 1412, 003 (2014) doi:10.1007/JHEP12(2014)003 [arXiv:1404.0711 [hep-th]].

[37] F. Apruzzi, M. Fazzi, A. Passias, D. Rosa and A. Tomasiello, JHEP 1411, 099 (2014) Erratum: [JHEP 1505, 012 (2015)] [arXiv:1406.0852 [hep-th]].

[38] F. Apruzzi, M. Fazzi, A. Passias, A. Rota and A. Tomasiello, Phys. Rev. Lett. 115, no. 6, 061601 (2015) doi:10.1103/PhysRevLett.115.061601 [arXiv:1502.06616 [hep-th]].

[39] A. Passias, A. Rota and A. Tomasiello, JHEP 1510, 187 (2015) doi:10.1007/JHEP10(2015)187 [arXiv:1506.05462 [hep-th]].

[40] F. Apruzzi and M. Fazzi, JHEP 1801, 124 (2018) [arXiv:1712.03235 [hep-th]].

[41] N. T. Macpherson and A. Tomasiello, JHEP $\mathbf{1 7 0 9 ,} 126 \quad$ (2017) doi:10.1007/JHEP09(2017)126 [arXiv:1612.06885 [hep-th]].

[42] N. Bobev, G. Dibitetto, F. F. Gautason and B. Truijen, JHEP 1702, 116 (2017) doi:10.1007/JHEP02(2017)116 [arXiv:1612.06324 [hep-th]].

[43] S. Cremonesi and A. Tomasiello, JHEP 1605, 031 (2016) doi:10.1007/JHEP05(2016)031 [arXiv:1512.02225 [hep-th]].

[44] K. Zarembo, doi:10.1093/oso/9780198828150.003.0005 [arXiv:1712.07725 [hep-th]]. 
[45] J. J. Kovacic, "An algorithm for solving second order homogeneous differential equations", J. Symb. Comp 2 (1986) 3-43

[46] L. A. Pando Zayas and C. A. Terrero-Escalante, JHEP 1009, 094 (2010) doi:10.1007/JHEP09(2010)094 [arXiv:1007.0277 [hep-th]].

[47] P. Basu and L. A. Pando Zayas, Phys. Rev. D 84, 046006 (2011) doi:10.1103/PhysRevD.84.046006 [arXiv:1105.2540 [hep-th]].

[48] P. Basu, D. Das, A. Ghosh and L. A. Pando Zayas, JHEP 1205, 077 (2012) doi:10.1007/JHEP05(2012)077 [arXiv:1201.5634 [hep-th]].

[49] Y. Chervonyi and O. Lunin, JHEP 1402, 061 (2014) doi:10.1007/JHEP02(2014)061 [arXiv:1311.1521 [hep-th]].

[50] A. Stepanchuk and A. A. Tseytlin, J. Phys. A 46, 125401 (2013) doi:10.1088/17518113/46/12/125401 [arXiv:1211.3727 [hep-th]].

[51] D. Giataganas, L. A. Pando Zayas and K. Zoubos, JHEP 1401, 129 (2014) doi:10.1007/JHEP01(2014)129 [arXiv:1311.3241 [hep-th]].

[52] D. Giataganas and K. Sfetsos, JHEP 1406, 018 (2014) doi:10.1007/JHEP06(2014)018 [arXiv:1403.2703 [hep-th]].

[53] Y. Asano, D. Kawai and K. Yoshida, JHEP 1506, 191 (2015) doi:10.1007/JHEP06(2015)191 [arXiv:1503.04594 [hep-th]].

[54] T. Ishii, K. Murata and K. Yoshida, Phys. Rev. D 95, no. 6, 066019 (2017) doi:10.1103/PhysRevD.95.066019 [arXiv:1610.05833 [hep-th]].

[55] K. Hashimoto, K. Murata and K. Yoshida, Phys. Rev. Lett. 117, no. 23, 231602 (2016) doi:10.1103/PhysRevLett.117.231602 [arXiv:1605.08124 [hep-th]].

[56] D. Giataganas and K. Zoubos, JHEP 1710, 042 (2017) doi:10.1007/JHEP10(2017)042 [arXiv:1707.04033 [hep-th]].

[57] D. Roychowdhury, JHEP 1710, 056 (2017) doi:10.1007/JHEP10(2017)056 [arXiv:1707.07172 [hep-th]].

[58] A. Banerjee and A. Bhattacharyya, JHEP 1811, $124 \quad$ (2018) doi:10.1007/JHEP11(2018)124 [arXiv:1806.10924 [hep-th]].

[59] D. Roychowdhury, JHEP 1909, 002 (2019) doi:10.1007/JHEP09(2019)002 [arXiv:1907.00584 [hep-th]]. 
[60] T. Akutagawa, K. Hashimoto, K. Murata and T. Ota, Phys. Rev. D 100, no. 4, 046009 (2019) doi:10.1103/PhysRevD.100.046009 [arXiv:1903.04718 [hep-th]].

[61] C. Nunez, J. M. Penin, D. Roychowdhury and J. Van Gorsel, JHEP 1806, 078 (2018) doi:10.1007/JHEP06(2018)078 [arXiv:1802.04269 [hep-th]].

[62] C. Nunez, D. Roychowdhury and D. C. Thompson, JHEP 1807, 044 (2018) doi:10.1007/JHEP07(2018)044 [arXiv:1804.08621 [hep-th]].

[63] L. Wulff, JHEP 1904, 133 (2019) doi:10.1007/JHEP04(2019)133 [arXiv:1903.08660 [hep-th]].

[64] L. Wulff, Phys. Rev. D 96, no. 10, 101901 (2017) doi:10.1103/PhysRevD.96.101901 [arXiv:1708.09673 [hep-th]].

[65] L. Wulff, JHEP 1802, 106 (2018) doi:10.1007/JHEP02(2018)106 [arXiv:1711.00296 [hep-th]].

[66] L. Wulff, J. Phys. A 50, no. 23, 23LT01 (2017) doi:10.1088/1751-8121/aa70b5 [arXiv:1702.08788 [hep-th]].

[67] D. Giataganas, arXiv:1909.02577 [hep-th].

[68] A. Hanany and E. Witten, Nucl. Phys. B 492, 152 (1997) doi:10.1016/S05503213(97)00157-0, 10.1016/S0550-3213(97)80030-2 [hep-th/9611230].

[69] S. Speziali, JHEP 03 (2020), 079 doi:10.1007/JHEP03(2020)079 [arXiv:1910.14390 [hepth]].

[70] G. Dibitetto and N. Petri, JHEP 1801, 039 (2018) doi:10.1007/JHEP01(2018)039 [arXiv:1707.06154 [hep-th]].

[71] G. Dibitetto and N. Petri, JHEP 1901, 193 (2019) doi:10.1007/JHEP01(2019)193 [arXiv:1807.07768 [hep-th]].

[72] A. Hanany and T. Okazaki, JHEP 1903, 027 (2019) doi:10.1007/JHEP03(2019)027 [arXiv:1811.09117 [hep-th]].

[73] D. Marolf, [arXiv:hep-th/0006117 [hep-th]].

[74] A. Babichenko, B. Stefanski, Jr. and K. Zarembo, JHEP 1003, 058 (2010) doi:10.1007/JHEP03(2010)058 [arXiv:0912.1723 [hep-th]].

[75] K. Sfetsos and D. C. Thompson, Nucl. Phys. B 846, 21 (2011) doi:10.1016/j.nuclphysb.2010.12.013 [arXiv:1012.1320 [hep-th]]. 
[76] D. Gaiotto and J. Maldacena, JHEP 1210, 189 (2012) doi:10.1007/JHEP10(2012)189 [arXiv:0904.4466 [hep-th]].

[77] F. Apruzzi, M. Fazzi, D. Rosa and A. Tomasiello, JHEP 1404, 064 (2014) doi:10.1007/JHEP04(2014)064 [arXiv:1309.2949 [hep-th]].

[78] P. Benetti Genolini and P. Richmond, JHEP 1910, 115 (2019) doi:10.1007/JHEP10(2019)115 [arXiv:1907.12561 [hep-th]].

[79] N. A. Nekrasov, Adv. Theor. Math. Phys. 7, no. 5, 831 (2003) doi:10.4310/ATMP.2003.v7.n5.a4 [hep-th/0206161].

[80] N. A. Nekrasov and S. L. Shatashvili, doi:10.1142/9789814304634-0015 arXiv:0908.4052 [hep-th].

[81] L. F. Alday, D. Gaiotto and Y. Tachikawa, Lett. Math. Phys. 91, 167 (2010) doi:10.1007/s11005-010-0369-5 [arXiv:0906.3219 [hep-th]].

[82] N. A. Nekrasov and S. L. Shatashvili, Prog. Theor. Phys. Suppl. 177, 105 (2009) doi:10.1143/PTPS.177.105 [arXiv:0901.4748 [hep-th]].

[83] N. Nekrasov and E. Witten, JHEP 1009, 092 (2010) doi:10.1007/JHEP09(2010)092 [arXiv:1002.0888 [hep-th]].

[84] D. Fioravanti and D. Gregori, arXiv:1908.08030 [hep-th].

[85] D. Orlando, S. Reffert, Y. Sekiguchi and K. Yoshida, J. Phys. A 51, no. 39, 395401 (2018) doi:10.1088/1751-8121/aad8c2 [arXiv:1805.00948 [hep-th]].

[86] Y. Sekiguchi, PoS CORFU 2018, 118 (2019). doi:10.22323/1.347.0118

[87] S. Hellerman, D. Orlando and S. Reffert, JHEP 1201, 148 (2012) doi:10.1007/JHEP01(2012)148 [arXiv:1106.0279 [hep-th]].

[88] S. Reffert, JHEP 1204 059(2012) doi: 10.1007/JHEP04(2012)059 [arXiv:1108.0644 [hep-th]].

[89] S. Hellerman, D. Orlando and S. Reffert, JHEP 1207, 061 (2012) doi:10.1007/JHEP07(2012)061 [arXiv:1204.4192 [hep-th]].

[90] N. Hama and K. Hosomichi, JHEP 1209, 033 (2012) Addendum: [JHEP 1210, 051 (2012)] doi:10.1007/JHEP09(2012)033, 10.1007/JHEP10(2012)051 [arXiv:1206.6359 [hep-th]].

[91] C. Klare and A. Zaffaroni, JHEP 1310, 218 (2013) doi:10.1007/JHEP10(2013)218 [arXiv:1308.1102 [hep-th]]. 
[92] C. M. Hull, JHEP 9807, 021 (1998) doi:10.1088/1126-6708/1998/07/021 [hepth/9806146].

[93] C. M. Hull, JHEP 9811, 017 (1998) doi:10.1088/1126-6708/1998/11/017 [hepth/9807127].

[94] S. Dobashi, H. Shimada and T. Yoneya, Nucl. Phys. B 665, 94 (2003) doi:10.1016/S05503213(03)00460-7 [hep-th/0209251].

[95] N. Seiberg and E. Witten, Nucl. Phys. B 471, 121 (1996) doi:10.1016/05503213(96)00189-7 [hep-th/9603003].

[96] J. D. Blum and K. A. Intriligator, Nucl. Phys. B 506, 199 (1997) [hep-th/9705044].

[97] I. Brunner and A. Karch, JHEP 9803, 003 (1998) doi:10.1088/1126-6708/1998/03/003 [hep-th/9712143].

[98] K. Ohmori, H. Shimizu and Y. Tachikawa, JHEP 1408, 002 (2014) doi:10.1007/JHEP08(2014)002 [arXiv:1404.3887 [hep-th]].

[99] M. Del Zotto, J. J. Heckman, A. Tomasiello and C. Vafa, JHEP 1502, 054 (2015) doi:10.1007/JHEP02(2015)054 [arXiv:1407.6359 [hep-th]].

[100] C. Cordova, T. T. Dumitrescu and K. Intriligator, JHEP 1610, 080 (2016) doi:10.1007/JHEP10(2016)080 [arXiv:1506.03807 [hep-th]].

[101] K. Intriligator, JHEP 1410, $162 \quad(2014)$ doi:10.1007/JHEP10(2014)162 [arXiv:1408.6745 [hep-th]].

[102] K. Ohmori, H. Shimizu, Y. Tachikawa and K. Yonekura, PTEP 2014, no. 10, 103 B07 (2014) doi:10.1093/ptep/ptu140 [arXiv:1408.5572 [hep-th]].

[103] M. Beccaria and A. A. Tseytlin, JHEP 1601, 001 (2016) doi:10.1007/JHEP01(2016)001 [arXiv:1510.02685 [hep-th]].

[104] L. Bhardwaj, JHEP 1511, $002 \quad$ (2015) doi:10.1007/JHEP11(2015)002 [arXiv:1502.06594 [hep-th]].

[105] I. Bandos, H. Samtleben and D. Sorokin, Phys. Rev. D 88, no. 2, 025024 (2013) doi:10.1103/PhysRevD.88.025024 [arXiv:1305.1304 [hep-th]].

[106] C. M. Chang, JHEP 09 (2019), 016 doi:10.1007/JHEP09(2019)016 [arXiv:1810.04169 [hep-th]].

[107] N. Seiberg, Phys. Lett. B 390, 169 (1997) doi:10.1016/S0370-2693(96)01424-4 [hepth/9609161]. 
[108] U. H. Danielsson, G. Ferretti, J. Kalkkinen and P. Stjernberg, Phys. Lett. B 405, 265 (1997) doi:10.1016/S0370-2693(97)00645-X [hep-th/9703098].

[109] A. Hanany and A. Zaffaroni, Nucl. Phys. B 529, 180 (1998) doi:10.1016/S05503213(98)00355-1 [hep-th/9712145].

[110] W. Nahm, Nucl. Phys. B 135, 149 (1978). doi:10.1016/0550-3213(78)90218-3 S. Minwalla, Adv. Theor. Math. Phys. 2, 783 (1998) doi:10.4310/ATMP.1998.v2.n4.a4 [hepth/9712074].

[111] C. Núñez, D. Roychowdhury, S. Speziali and S. Zacarías, Nucl. Phys. B 943 (2019), 114617 doi:10.1016/j.nuclphysb.2019.114617 [arXiv:1901.02888 [hep-th]].

[112] I. R. Klebanov, D. Kutasov and A. Murugan, Nucl. Phys. B 796 (2008), 274-293 doi:10.1016/j.nuclphysb.2007.12.017 [arXiv:0709.2140 [hep-th]].

[113] S. Ryu and T. Takayanagi, Phys. Rev. Lett. 96 (2006), 181602 doi:10.1103/PhysRevLett.96.181602 [arXiv:hep-th/0603001 [hep-th]].

[114] U. Kol, C. Nunez, D. Schofield, J. Sonnenschein and M. Warschawski, JHEP 1406, 005 (2014) doi:10.1007/JHEP06(2014)005 [arXiv:1403.2721 [hep-th]]. N. T. Macpherson, C. Nunez, L. A. Pando Zayas, V. G. J. Rodgers and C. A. Whiting, JHEP 1502, 040 (2015) doi:10.1007/JHEP02(2015)040 [arXiv:1410.2650 [hep-th]].

[115] Y. Bea, J. D. Edelstein, G. Itsios, K. S. Kooner, C. Nunez, D. Schofield and J. A. SierraGarcia, JHEP 05 (2015), 062 doi:10.1007/JHEP05(2015)062 [arXiv:1503.07527 [hepth]].

[116] K. Sfetsos and D. C. Thompson, JHEP 1412, 164 (2014) doi:10.1007/JHEP12(2014)164 [arXiv:1410.1886 [hep-th]]. S. Driezen, A. Sevrin and D. C. Thompson, JHEP 1809, 015 (2018) doi:10.1007/JHEP09(2018)015 [arXiv:1806.10712 [hep-th]].

[117] G. Arutyunov and S. Frolov, JHEP 0502, 059 (2005) doi:10.1088/11266708/2005/02/059 [hep-th/0411089].

[118] Y. Lozano, E. Ó Colgáin, D. Rodríguez-Gómez and K. Sfetsos, Phys. Rev. Lett. 110 (2013) no.23, 231601 doi:10.1103/PhysRevLett.110.231601 [arXiv:1212.1043 [hep-th]].

[119] E. D'Hoker, M. Gutperle, A. Karch and C. F. Uhlemann, JHEP 08 (2016), 046 doi:10.1007/JHEP08(2016)046 [arXiv:1606.01254 [hep-th]].

[120] E. D'Hoker, M. Gutperle and C. F. Uhlemann, Phys. Rev. Lett. 118 (2017) no.10, 101601 doi:10.1103/PhysRevLett.118.101601 [arXiv:1611.09411 [hep-th]]. 
[121] E. D'Hoker, M. Gutperle and C. F. Uhlemann, JHEP 05 (2017), 131 doi:10.1007/JHEP05(2017)131 [arXiv:1703.08186 [hep-th]].

[122] M. Gutperle, A. Trivella and C. F. Uhlemann, JHEP 04 (2018), 135 doi:10.1007/JHEP04(2018)135 [arXiv:1802.07274 [hep-th]].

[123] M. Fluder and C. F. Uhlemann, Phys. Rev. Lett. 121 (2018) no.17, 171603 doi:10.1103/PhysRevLett.121.171603 [arXiv:1806.08374 [hep-th]].

[124] O. Bergman, D. Rodríguez-Gómez and C. F. Uhlemann, JHEP 08 (2018), 127 doi:10.1007/JHEP08(2018)127 [arXiv:1806.07898 [hep-th]].

[125] Y. Lozano, N. T. Macpherson and J. Montero, JHEP 01 (2019), 116 doi:10.1007/JHEP01(2019)116 [arXiv:1810.08093 [hep-th]].

[126] D. Gaiotto, JHEP 08 (2012), 034 doi:10.1007/JHEP08(2012)034 [arXiv:0904.2715 [hep-th]].

[127] R. A. Reid-Edwards and B. Stefanski, jr., Nucl. Phys. B 849 (2011), 549-572 doi:10.1016/j.nuclphysb.2011.04.002 [arXiv:1011.0216 [hep-th]].

[128] O. Aharony, L. Berdichevsky and M. Berkooz, JHEP 08 (2012), 131 doi:10.1007/JHEP08(2012)131 [arXiv:1206.5916 [hep-th]].

[129] I. Bah, JHEP 09 (2015), 163 doi:10.1007/JHEP09(2015)163 [arXiv:1501.06072 [hepth]].

[130] I. Bah, F. Bonetti, R. Minasian and E. Nardoni, JHEP 06 (2019), 123 doi:10.1007/JHEP06(2019)123 [arXiv:1904.07250 [hep-th]].

[131] D. Gaiotto and E. Witten, Adv. Theor. Math. Phys. 13 (2009) no.3, 721-896 doi:10.4310/ATMP.2009.v13.n3.a5 [arXiv:0807.3720 [hep-th]].

[132] E. D'Hoker, J. Estes and M. Gutperle, JHEP 06 (2007), 022 doi:10.1088/11266708/2007/06/022 [arXiv:0705.0024 [hep-th]].

[133] E. D'Hoker, J. Estes, M. Gutperle and D. Krym, JHEP 08 (2008), 028 doi:10.1088/1126-6708/2008/08/028 [arXiv:0806.0605 [hep-th]].

[134] B. Assel, C. Bachas, J. Estes and J. Gomis, JHEP 08 (2011), 087 doi:10.1007/JHEP08(2011)087 [arXiv:1106.4253 [hep-th]].

[135] Y. Lozano, N. T. Macpherson, J. Montero and C. Nunez, JHEP 11 (2016), 133 doi:10.1007/JHEP11(2016)133 [arXiv:1609.09061 [hep-th]]. 
[136] E. Witten, JHEP 07 (1997), 003 doi:10.1088/1126-6708/1997/07/003 [arXiv:hepth/9707093 [hep-th]].

[137] N. Seiberg and E. Witten, JHEP 04 (1999), 017 doi:10.1088/1126-6708/1999/04/017 [arXiv:hep-th/9903224 [hep-th]].

[138] D. Kutasov, F. Larsen and R. G. Leigh, Nucl. Phys. B 550 (1999), 183-213 doi:10.1016/S0550-3213(99)00144-3 [arXiv:hep-th/9812027 [hep-th]].

[139] J. M. Maldacena, A. Strominger and E. Witten, JHEP 12 (1997), 002 doi:10.1088/1126-6708/1997/12/002 [arXiv:hep-th/9711053 [hep-th]].

[140] D. Martelli and J. Sparks, Phys. Rev. D $68 \quad$ (2003), 085014 doi:10.1103/PhysRevD.68.085014 [arXiv:hep-th/0306225 [hep-th]].

[141] A. Castro, J. L. Davis, P. Kraus and F. Larsen, Int. J. Mod. Phys. A 23 (2008), 613-691 doi:10.1142/S0217751X08039724 [arXiv:0801.1863 [hep-th]].

[142] C. Couzens, H. het Lam, K. Mayer and S. Vandoren, JHEP 08 (2019), 043 doi:10.1007/JHEP08(2019)043 [arXiv:1904.05361 [hep-th]].

[143] J. Kim, S. Kim and K. Lee, JHEP 02 (2016), 170 doi:10.1007/JHEP02(2016)170 [arXiv:1503.07277 [hep-th]].

[144] A. Gadde, B. Haghighat, J. Kim, S. Kim, G. Lockhart and C. Vafa, JHEP 02 (2018), 143 doi:10.1007/JHEP02(2018)143 [arXiv:1504.04614 [hep-th]].

[145] C. Lawrie, S. Schafer-Nameki and T. Weigand, JHEP 04 (2017), 111 doi:10.1007/JHEP04(2017)111 [arXiv:1612.05640 [hep-th]].

[146] C. Couzens, C. Lawrie, D. Martelli, S. Schafer-Nameki and J. M. Wong, JHEP 08 (2017), 043 doi:10.1007/JHEP08(2017)043 [arXiv:1705.04679 [hep-th]].

[147] Y. Lozano, N. T. Macpherson, J. Montero and E. Ó. Colgáin, JHEP 08 (2015), 121 doi:10.1007/JHEP08(2015)121 [arXiv:1507.02659 [hep-th]].

[148] Ö. Kelekci, Y. Lozano, J. Montero, E. Ó. Colgáin and M. Park, Phys. Rev. D 93 (2016) no.8, 086010 doi:10.1103/PhysRevD.93.086010 [arXiv:1602.02802 [hep-th]].

[149] N. T. Macpherson, JHEP 05 (2019), 089 doi:10.1007/JHEP05(2019)089 [arXiv:1812.10172 [hep-th]].

[150] A. Legramandi and N. T. Macpherson, Fortsch. Phys. 68 (2020) no.3-4, 2000014 doi:10.1002/prop.202000014 [arXiv:1912.10509 [hep-th]]. 
[151] Y. Lozano, C. Nunez, A. Ramirez and S. Speziali, [arXiv:2005.06561 [hep-th]].

[152] L. Alvarez-Gaume and E. Witten, Nucl. Phys. B 234 (1984), 269 doi:10.1016/05503213(84)90066-X

[153] F. Benini and N. Bobev, JHEP 06 (2013), 005 doi:10.1007/JHEP06(2013)005 [arXiv:1302.4451 [hep-th]].

[154] P. Putrov, J. Song and W. Yan, JHEP 03 (2016), 185 doi:10.1007/JHEP03(2016)185 [arXiv:1505.07110 [hep-th]].

[155] E. Witten, AMS/IP Stud. Adv. Math. 1 (1996), 143-211 doi:10.1016/05503213(93)90033-L [arXiv:hep-th/9301042 [hep-th]].

[156] D. Tong, JHEP 04 (2014), 193 doi:10.1007/JHEP04(2014)193 [arXiv:1402.5135 [hep$\operatorname{th}]$.

[157] R. C. Myers, JHEP 12 (1999), 022 doi:10.1088/1126-6708/1999/12/022 [arXiv:hepth/9910053 [hep-th]].

[158] O. Bergman, M. Fazzi, D. Rodríguez-Gómez and A. Tomasiello, JHEP 05 (2020), 138 doi:10.1007/JHEP05(2020)138 [arXiv:2002.04036 [hep-th]].

[159] J. Wess and J. Bagger, (1992) "Supersymmetry and supergravity,"

[160] P. C. West, "Introduction to rigid supersymmetric theories," In *Cambridge 1997, Confinement, duality, and nonperturbative aspects of QCD* 453-476 [hep-th/9805055]. 